
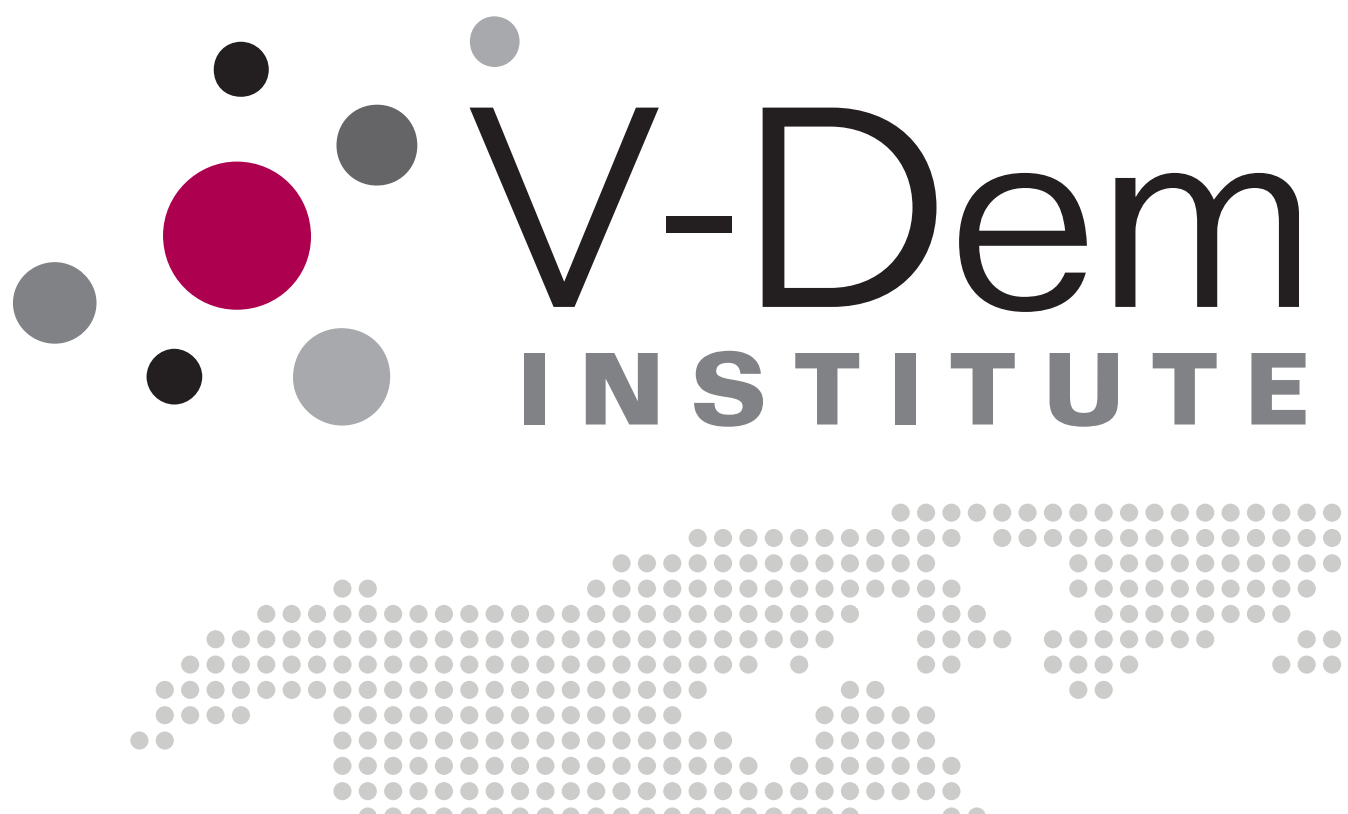

Multiple Measurements, Elusive Agreement, and Unstable Outcomes in the Study of Regime Change

Hans Lueders Ellen Lust

00000000

0000000

000000

100000

10000

000

000

10

10

90
100000

00000

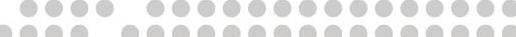

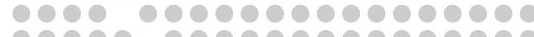

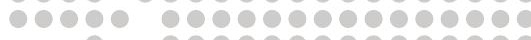
-

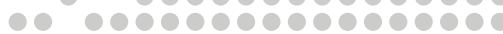

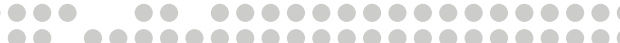
מ

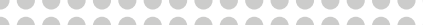

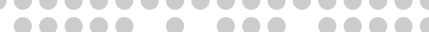

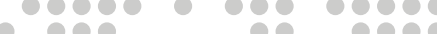
60

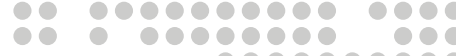

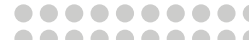

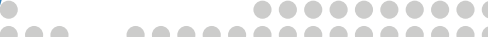

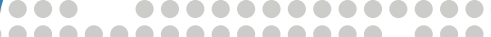

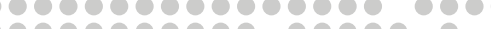
60000008000 00000

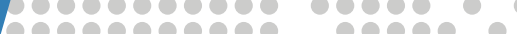

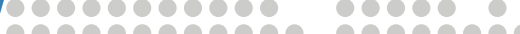

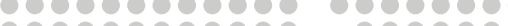

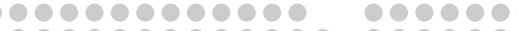
60000000000000 00000 1000000000000 1000000000000000

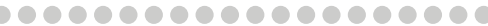
00000000000000000000 2000000000090000000

0000000000000

P0000000000000

000000000000

0000000000

0009000000

000000000

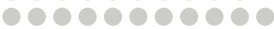

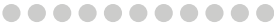

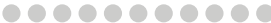

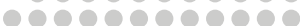

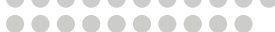

reserose

000000000

0000000

100000

000000

1000

100 10 
Varieties of Democracy (V-Dem) is a new approach to conceptualization and measurement of democracy. It is co-hosted by the University of Gothenburg and University of Notre Dame. With a V-Dem Institute at University of Gothenburg with almost ten staff, and a project team across the world with four Principal Investigators, fifteen Project Managers (PMs), 30+ Regional Managers, 170 Country Coordinators, Research Assistants, and 2,500 Country Experts, the VDem project is one of the largest ever social science research-oriented data collection programs.

Please address comments and/or queries for information to:

V-Dem Institute

Department of Political Science

University of Gothenburg

Sprängkullsgatan 19, PO Box 711

SE 40530 Gothenburg

Sweden

E-mail: contact@v-dem.net

V-Dem Working Papers are available in electronic format at www.v-dem.net.

Copyright C 2017 University of Gothenburg, V-Dem Institute. All rights reserved. 


\title{
Multiple Measurements, Elusive Agreement, and Unstable Outcomes in the Study of Regime Change*
}

\author{
Hans Lueders \\ PhD Candidate \\ Stanford University \\ Ellen Lust \\ Professor \\ University of Gothenburg
}

\footnotetext{
* We thank Matt Buehler, Lindsay Hundley, Jana Morgan, Michael Touchton, Jeremy Wallace, and participants at the 2015 APSA, 2016 SPSA, and 2016 MPSA meetings for helpful comments. We gratefully acknowledge collaboration with David Waldner and support of the USAID/IIE Grant, "Unwelcome Change: Understanding, Examining and Extending Theories of Democratic Backsliding," which supported development of this paper. Special thanks to colleagues who shared their data with us and provided helpful feedback on the paper, and to Staffan Lindberg and the Varieties of Democracy Institute for including these findings in the working paper series.
} 


\begin{abstract}
This comprehensive analysis of regime change indicators reveals that problems of conceptualization and measurement are major reasons why current research fails to draw compelling conclusions that foster cumulative knowledge. The paper first argues that even though the literature discusses the conceptualization of regime types at length, there is little attention to defining regime change. Furthermore, quantitative studies of regime change largely elide conceptual and measurement challenges. Second, although indicators of regime type are highly correlated, agreement between indicators of regime change is extremely low. Third, focal points such as elections and coups drive agreement among these indicators, suggesting that such measures often reflect notable events instead of regime change per se. Finally, a robustness check of nine articles on regime change published in top journals demonstrates that findings are often not robust to alternative indicators, implying that indicator choice influences the results of quantitative studies.
\end{abstract}




\section{Introduction}

A striking level of instability witnessed after the end of the Cold War has turned our attention to regime change. For instance, the Baltic states and more recently Tunisia witnessed democratization, while countries such as Hungary or Russia-which once experienced liberalization-are made increasingly authoritarian through "executive aggrandizement" (Bermeo 2016). This prompts renewed interest in regime change and spurs new studies in the large and intellectually vibrant literatures regarding the causes and consequences of democratic transitions, democratic breakdowns, and authoritarian resilience.

These studies are often methodologically sophisticated but yield few consistent findings. For instance, some claim that economic inequality inhibits democratization (Muller 1995; Boix 2003; Acemoglu and Robinson 2006), while others find no evidence for this relationship (Przeworski et al. 2000; Houle 2009). Presidentialism increases the likelihood of democratic breakdown according to some scholars (Przeworski et al. 2000; Maeda 2010), or has no effect (Power and Gasiorowski 1997; Cheibub 2007; Houle 2009). In short, an enormous literature has yielded few axiomatic findings.

This paper argues that the absence of rigorous, consistent conceptualization and measurement of regime change helps explain the lack of cumulative knowledge. It joins work by other scholars who have critiqued measurements of regime change, noting that they fail to reflect democratic ideals fully (Bogaards 2010; Paxton 2000) and yield inconsistent findings on outcomes from democratization to war (Casper and Tufis 2003; Bogaards 2010). However, it advances these studies by providing a more comprehensive review of existing measures and their implications for the study of regime change.

In this paper, we distinguish between regime rupture-large-scale changes between democracy and autocracy that fundamentally affect the logic of political rule — and reform — more fine-grained changes in a polity's institutional arrangements-and examine numerous indicators of regime change derived from 13 original datasets on regime type. We demonstrate that the problem of measurement is more worrisome than previous work suggests: indicators count vastly different numbers of regime change events over time, and agreement between these indicators is extraordinarily low. Moreover, we find that elections and coups increase agreement among indicators, suggesting that where agreement exists, it is driven by highly visible symbols of regime change. Finally, a robustness check of nine articles on regime change published in top journals reveals that findings are often not robust to the use of alternative measures. 
Our goal is to raise awareness of the consequences of indicator choice on scholarly findings. The robustness checks of our colleagues' research are not intended as a critique of their work or the theories they put forth, but rather to underscore the lack of interchangeability of conventionally used indicators. We highlight the methodological problems that make it difficult to draw conclusions from current scholarship. We do not aim to explain regime change or to argue that a particular type of indicator is more appropriate than another. Rather, we point to the factors that scholars should more explicitly consider in testing hypotheses, and urge the research community to more rigorously conceptualize and measure regime change in future work.

The paper is structured as follows. Section II discusses challenges in the conceptualization and measurement of different forms of regime change. Section III reviews common measures of regime change. Section IV examines agreement between them. Section V analyzes the robustness of empirical studies to alternative indicators. Section VI suggests some improvements for empirical research. Section VII discusses our contributions and concludes.

\section{Conceptualizing and Measuring Regime Change}

\section{Conceptual Challenges}

Challenges in conceptualizing and measuring regime change derive from the debates over the conceptualization and measurement of regime type, particularly democracy. Scholars disagree about the definition of democracy. Specifically, they are at odds over whether to adopt a binary concept of democracy and dictatorship or to see democracy on a continum. Those who advocate the binary definition argue that democracy, like pregnancy, reflects a fundamental, qualitative change in condition. A regime is either democratic or not. Typically, these scholars rely on a procedural definition of democracy emphasizing competitive elections for executive and legislative offices in which multiple parties compete and alternation in office between parties is observed (Alvarez et al. 1996; Cheibub et al. 2010). Those who advocate a continuum take the view that regimes exhibit democratic qualities to a greater or lesser extent (Dahl 1971; Bollen and Jackman 1989; Diamond 1999).

This debate reflects two very different ways in which political regime change is conceptualized. For those who conceive of regime types in binary terms and only distinguish between democracies and autocracies, any change from democracy to autocracy or vice versa constitutes a large-scale change in the logic of politics. We call this form of regime change rupture. On the other hand, a perspective that conceptualizes political regimes along a continuum between two ideal types or that distinguishes between various distinct forms of democracy, 
autocracy, and hybrid regimes, allows for what we term reform: more fine-grained changes that may affect single political rights, civil liberties, or some institutional characteristics, but do not imply a complete overhaul of the system. Both rupture and reform come in two flavors. As to rupture, countries may change from a democratic to an autocratic political order (breakdown) or vice versa (transition). Liberalization is a reform that entails an increase in a country's democratic qualities. Backsliding decreases these qualities.

The pace and magnitude of institutional changes distinguish rupture and reform. Gradual institutional changes over a long period of time characterize reform, while large, abrupt changes classify as rupture. Contrast, for example, the political rupture in Peru under Alberto Fujimori with the reform in Russia under Vladimir Putin. On April 5, 1992, Alberto Fujimori responded to political deadlock by suspending the constitution, removing adversaries from the judiciary, and dissolving Congress. In the aftermath of this autogolpe, Fujimori established an autocratic regime that ensured his reelection in 1995 and 2000 (Cameron and Mauceri 2006; Seawright 2012). Thus, Peru saw abrupt and large-scale institutional changes that characterize this regime change event as rupture. Putin's process of regime change, by contrast, constitutes an example of reform. Putin gradually rolled back democratic freedoms by passing more restrictive media laws in 2005, cracking down on political opponents through an anti-terrorism law in 2006, and placing greater sanctions on public assemblies, NGOs, and the internet in 2011. In a move to de facto circumvent constitutional term limits, Putin installed his then prime minister, Dmitry Medvedev, as President from 2008-2012, while himself serving as prime minister, and then pushed for the extension of presidential term limits to six years before his return to the presidency in 2012 (Freedom House various years). It is hard to identify any specific moment that marked the transition from democracy to autocracy in this process of reform.

\section{Measurement Challenges}

Measurement issues have received a great deal of attention in the study of regimes, but there is little explicit attention given to measuring regime change. In our view, the challenge in studying regime change is to define and measure it in a way that allows us to detect both rupture and reform, and both minimalist and maximalist definitions of democracy present challenges in this regard. A minimalist focus on turnover through elections (or any other single aspect of democracy that signals regime change), often advocated by those who see democracy in binary terms, cannot detect fine-grained reforms because it only allows for changes between the democratic and autocratic ideal types. A maximalist view that requires highly informed citizens to engage in near-constant deliberation to produce policies that maximize social, economic, and 
cultural equality, is equally problematic, because it makes it extremely unlikely that regimes pass the threshold to democracy and, thus, difficult to detect rupture.

A related challenge regards determining the relevant time period for measuring rupture and reform. Several authors designed their data on the premise that regime change is "marked by singular, characteristic events, such as free or fraudulent elections, constitutional changes, coups d'état, declarations of martial law, or arrests of prominent individuals" (Gasiorowski 1996: 472), yet the changes in the rules and informal procedures that shape political regimes take place over time, separated by months or even years. Moreover, not all crises, no matter how startling, denote regime change. Further, it is doubtful that such events should be seen as the "moment" of regime change. The relevant changes often begin long before troops leave the barracks or elections are called. Understanding which changes constitute reform or rupture is thus difficult.

Another measurement challenge is to avoid excessively including cases of political change and crises that fall short of significant changes in the democratic qualities of political regimes. For instance, tighter restrictions on press freedoms, including some prosecution of independent journalists, may be deeply unsettling, and they may also be early warning signs of more systemic efforts of backsliding. However, practices that are limited in scope may not be sufficient to characterize the event as backsliding. Similarly, the expansion of press freedoms or crackdowns on corruption may be a welcome change but they do not necessarily reflect liberalization or transition. Such changes can be the normal push and pull of politics; they are near ubiquitous and can be observed even in advanced and seemingly stable democracies.

Conversely, not all significant changes are immediately obvious. Sometimes regime changes result from dramatic political events-tanks rolling into the streets to oust a democratically elected president or the presidential suspension of an elected legislature, on the one hand; the election of a president through free and fair competition, on the other. Yet, often they come gradually-whether through piecemeal restrictions of political and civil liberties, diminished accountability or electoral fraud, in the case of backsliding and breakdown, or through the slow expansion of liberties, extension of accountability and increasingly competitive elections, in the case of liberalization and transition. This makes it hard to identify one specific year of regime change, and absent highly visible changes, can lead scholars to overlook change altogether. It also draws into question how we should think about the time period that constitutes a regime change event. Following the conventional country-year design of extant datasets of regime types and empirical studies of regime change, we would identify several distinct years and thus potential cases of rupture or reform. However, it seems more adequate to interpret them as one multi-year period of change. 


\section{Extant Indicators of Regime Change}

Given these challenges, it is perhaps not surprising that published research on regime change often lacks a clear definition of the phenomenon under investigation. The literature on regime change relies on varied measures, often employing them out of convenience or for methodological reasons that have little to do with the underlying theory of political regime change. Even where scholars have carefully taken into account the underlying conceptions of regime type when choosing indicators, they often fail to consider explicitly the implications of using these measures as indicators of regime change.

Existing measurements create different challenges for studying rupture and reform. Dichotomous indicators cannot be used to analyze reform. Polychotomous or continuous indicators often do not identify qualitative thresholds between different forms of political rule, thus making it difficult to use them in analyses of rupture. They also combine factors that reflect different combinations of democratic features (e.g., accountability, electoral competition, and liberties), making it hard to isolate arenas of democratic or autocratic change.

\section{Datasets on Regime Type: Conceptual Differences}

Our review considers thirteen original datasets of regime type that are used to study regime change. We include twelve datasets that are used in published research on regime change. In addition, we consider the Varieties of Democracy (V-Dem) dataset (Coppedge et al. 2016a). This dataset was released in January 2016 and has not yet formed a basis of a body of published work. However, we include it because it is likely to become a leading basis for analysis in the field and because it explicitly recognizes and measures varied conceptions of democracy.

The indicators that we review differ with respect to their conceptualization of democracy. In particular, they disagree on whether to adopt a minimalist definition of democracy focusing on (electoral) contestation alone (Przeworski 1991), a notion of "polyarchy" involving both contestation and participation (Dahl 1971), or an even broader definition of democracy that adds civil and/or political liberties (Reich 2002; Freedom House 2015) to the concept of democracy.

These indicators can be grouped into one of three conceptualizations of democracy. Przeworski et al.'s (2000) dataset on regime type and its update by Cheibub et al. (2010) employs a minimalist concept of democracy, whereby democracy is "a regime in which some governmental offices are filled as a consequence of contested elections" (Alvarez et al. 1996). Contestation, thus, is central in this concept. Svolik (2012) adopts a similar approach. 
The second conceptualization adds other criteria. Boix et al. (2013), Geddes et al. (2014), and Skaaning et al. (2015) introduce participation as second core dimension, requiring countries to meet a minimal suffrage requirement to qualify as democratic (Dahl 1971). Bernhard et al. (2001) add a stateness criterion to contestation and participation: democratic countries must have achieved full sovereignty. Conceptually, the Polity IV data (Marshall et al. 2014) focus on contestation and participation as well. However, their measurement puts a particularly strong emphasis on political institutions and executive constraints as third dimension of political rule. Magaloni et al. (2013) formulate four necessary conditions of democracy: contestation, participation, a civilian government, and executive constraints.

The V-Dem project recognizes that the notion of "democracy" entails up to seven different principles: the electoral, liberal, participatory, deliberative, egalitarian, majoritarian, and consensual principles (Coppedge et al. 2016b). Thus far, separate indicators of the first five principles are available. The electoral democracy score is the foundation of the other four indicators because elections are seen as necessary condition for democracy. Consequently, including all five indicators in this study would bias our findings. Therefore, we only consider the first score. ${ }^{1}$ The project's definition of electoral democracy follows Dahl's (1998) concept of polyarchy and thus focuses on contestation and participation (Coppedge et al. 2015).

The remaining four datasets employ a broad concept of democracy. Reich $(2002)^{2}$ and Ulfelder (2012) examine a regime's level of contestation, participation, and civil and political liberties. Freedom House (2015) assesses a country's political rights (contestation and participation) and civil liberties (e.g., freedom of expression and belief; associational and organizational rights; rule of law; personal autonomy and individual rights). Wahman et al. (2013) use the imputed average Polity IV (Marshall et al. 2014) and Freedom in the World (Freedom House 2015) scores to measure political regimes. The authors derive the threshold between democratic and autocratic rule on their indicator empirically by estimating the mean cutoff point of five established democracy indicators (Bernhard et al. 2001; Cheibub et al. 2010; Boix et al. 2013; Freedom House's (2015) measure of “electoral democracy," and Polity IV). This identifies a cut-off point of 7 (out of 10).

\footnotetext{
1 The findings remain unaffected when we include all five V-Dem indicators (see Appendix).

2 Reich (2002) is an update of Gasiorowski (1996). As with Cheibub et al.'s (2010) update of the Przeworski et al. (2000) data, we include only the more comprehensive dataset in our analysis.
} 


\section{Measurements of Regime Change}

In selecting the indicators for our analysis we aim to include the most commonly used indicators of regime change and to reflect the breadth of indicators employed in the literature. Several datasets on regime type - in particular, Freedom House (2015), Polity IV, Skaaning et al. (2015), and Wahman et al. (2013) —allow for variable cutpoints between democracy and autocracy and different magnitudes of change, and our literature review revealed that scholars employ different thresholds on the same regime type indicator to code instances of rupture and reform. Yet, adding more than one indicator based on the same dataset may bias our findings. What is why we include only one indicator per type of regime change in this paper. We replicated all the analyses reported below with an extended set of indicators of regime change and present the findings in the Appendix. ${ }^{3}$

Our analysis covers eleven indicators of breakdown, ten indicators of transition, and five indicators of reform, based on the aforementioned 13 original datasets of regime type. ${ }^{4}$ As to rupture, six of the twelve indicators of regime type (Boix et al. 2013; Cheibub et al. 2010; Geddes et al. 2014; Magaloni et al. 2013; Svolik 2012; Ulfelder 2012) are dichotomous and identify clear qualitative thresholds between democratic and autocratic regimes. To these, we add Bernhard et al.'s (2001) original measure of democratic breakdown. Reich's (2002) data are trichotomous and distinguish between democracies, semidemocracies, and autocracies. Rupture is any move between democracy and autocracy. We use the commonly employed thresholds of +6 on the Polity IV score, 2.5 on the Freedom in the World index, and 7 on Wahman et al.'s (2013) indicator to derive three more indicators of rupture.

Regarding reform, the analysis includes five indicators. These are defined as any change on Freedom House's (2015) Freedom in the World index, the Polity IV indicator, Skaaning et al.'s (2015) Lexical Index of Electoral Democracy ${ }^{5}$, and Wahman et al.'s (2013) combined Freedom House and Polity score. Identifying regime change events using the V-Dem indicators is particularly challenging because the data are continuous between 0 and 1. To distinguish trivial from meaningful changes, we define a reform as any change on V-Dem's electoral democracy scale by at least a standard deviation. ${ }^{6}$

\footnotetext{
${ }^{3}$ Note that our indicator selection is conservative-agreement is even lower when additional commonly used indicators of regime change are used.

${ }^{4}$ See Appendix for an overview of all measures of regime change, their definition, sources, coding rules, and exemplary studies that use them.

${ }^{5}$ As Skaaning et al. (2015) do not provide guidelines of how to use their index to distinguish between democracies and autocracies, we do not include it among the rupture measures.

${ }^{6}$ Lindberg (2015) presents a way to transform the continuous into a five-point ordinal scale, using cutoff points at $0.2,0.4,0.6$, and 0.8 . Such an ordinal indicator can then be used to study reform as any change between these
} 


\section{Evaluating Extant Indicators of Regime Change}

We turn now to an evaluation of these indicators. We begin by considering measurement issues in Freedom House and Polity IV, given their importance in the literature. We then examine the congruence in measures, finding extremely low agreement in the number of instances of rupture and reform they identify. Finally we model the agreement of these measures and show that they are more likely to agree when focal points such as coups or elections are present. The purpose of modeling the agreement of indicators (which, we emphasize, is distinct from modeling rupture and reform), is to determine whether we can expect that coding processes-and particularly the existence of highly visible events - affect the data collection process and (in) congruence of extant indicators.

\section{Freedom House and Polity IV: Empirical Ambiguity}

Polity IV provides annual measures of both autocratic and democratic features according to five dimensions of the exercise of authority: competitiveness of political participation, regulation of political participation, competiveness of executive recruitment, openness of executive recruitment, and constraints on the chief executive. Its aggregate score ranges from -10 to +10 , with higher values corresponding to more "democratic quality" of the regime. Freedom House (2015) measures civil liberties and political rights. Both dimensions are coded on a seven-point scale with higher numbers corresponding to a higher number of restrictions imposed on the respective dimension.

Both indicators have been subject to several critiques. First, rules for coding individual elements and aggregating them into a single score are subjective and often opaque. ${ }^{7}$ Freedom House's 7-point political rights and civil liberties indices aggregate information on 3 (4) dimensions, which are coded on 40- (60-) point scales. These dimensions, in turn, summarize multiple questions. Coding information is only available at the dimension-, not at the individual question-level. Moreover, this information is only available from 2005 onward. Polity subcomponents, in contrast, are available for all countries and years.

Second, the various aggregation steps in the creation of the Freedom House indices imply a multitude of possible combinations of individual scores. At the question level, there are 9,765,625 logically possible combinations of the subcategory questions to obtain a plausible score on

categories. In the appendix, replicate all our analyses using reform measures based on the ordinal instead of continuous scales.

${ }^{7}$ See Munck and Verkuilen (2002), Casper and Tufis (2003), and Cheibub et al. (2010) for overviews. 
Freedom House's Political Rights scale. In turn, there are 30,517,578,125 combinations of the Civil Liberties questions. At the subcategory level, there are 2,873 (63,869) different logically plausible combinations for the political rights (civil liberties) indices.

Of course, most of the possible combinations are not found empirically. Yet, over the period 2005-2015 alone, we find a striking number of combinations in the data. Usually, there are at least 5-10 combinations of subcategory scores that yield the same aggregate score. For the 7-point scales, we find more than 25 combinations of questions per score in the data. Polity's aggregation rules have similar effects. We count 2,100 logically possible combinations of the five Polity component indicators. Again, the majority of them are empirically implausible. However, we find, for instance, nine different combinations for a score of -6 , and fourteen for -5 .

This leads to a third critique: changes in scores do not necessarily reflect similar underlying changes in liberties or institutional arrangements. For example, a change from -6 to -5 on Polity (that is, a change from autocracy to anocracy) could be from any of the nine combinations that result in a -6 to any of the 14 combinations that yield a score of -5 . That makes 84 different possible regime changes. Alternatively, a change from 2 (52 combinations in the data) to 3 (69 combinations in the data) on Freedom House's Political Rights scale could reflect any of 3,588 different movements. In short, any aggregate score on the Polity IV or Freedom in the World index represents a high number of underlying institutional characteristics, and movements between these categories provide only limited information.

Finally, the regime thresholds created on the basis of Polity IV or Freedom House are arbitrary. Freedom House takes the average of the Political Rights and Civil Liberties ratings to create a trichotomous Freedom Rating according to which countries can be Free (1.0 to 2.5), Partly Free (3.0 to 5.0) or Not Free (5.5 to 7.0). The addition of a third category appears to provide more information, but the majority of cases fall at the high or low ends-and they tend to remain there for longer periods of time. The middle category is where most regime changes take place, and this is where we need refined definitions and measures. Similarly, Polity IV's official cut-off points are -6 (autocracy) and +6 (democracy), with all countries in between defined as anocracies. Again, these thresholds are arbitrary, and given the high number of permutations of the component indicators that arrive at a specific score, movements across categories may represent very different institutional dynamics. 


\section{Coding Instances of Regime Change}

It is perhaps not surprising that indicators differ widely in terms of the number of regime change events identified, as Table 1 shows. Some discrepancies may be due to the different time periods covered-the likelihood of specific forms of regime change varies over time (e.g., Huntington 1992; Doorenspleet 2000). Nevertheless, even when the period is restricted to 1946-2000, for which most (with the exception of Freedom House) data are available, we find that disagreement about the number of country-years experiencing regime change is large. For example, Reich (2002) identifies 106 instances of transition in the post-WWII period (1.79\% of all observations), while Svolik (2012) codes only 89 observations (1.07\%). Similarly, Magaloni et al.'s (2013) data allow for the coding of 49 breakdown events after 1945 ( $0.58 \%$ of all country-years), while according to Ulfelder (2012), breakdown happened almost three times as frequently in the same period (109 events; $1.45 \%)$.

These discrepancies become even larger when measuring reform. For example, according to Skaaning et al. (2015), liberalization (backsliding) occurred in 379 (261) country-years after World War II. According to Polity IV, 556 (341) country-years experienced liberalization (backsliding).

Naturally, these discrepancies are also due to differences in the range of values of both indicators. But they also suggest that the set of cases examined in an analysis of rupture or reform, and thus any inferences about the determinants of regime change or its consequences, crucially depend on the chosen indicator of regime change. This implies that we cannot even answer questions as simple as about the number of regime changes after World War II. 
Table 1: Indicators of regime change

\begin{tabular}{|c|c|c|c|c|c|c|c|c|c|}
\hline \multirow[b]{2}{*}{ Indicator name } & \multirow[b]{2}{*}{$\begin{array}{c}\text { Period } \\
\text { available }\end{array}$} & \multicolumn{2}{|c|}{ full period } & \multicolumn{2}{|c|}{ post-WWII } & \multicolumn{2}{|c|}{ full period } & \multicolumn{2}{|c|}{ post-WWII } \\
\hline & & $\begin{array}{c}\# \\
\text { events }\end{array}$ & $\%$ obs & $\begin{array}{c}\# \\
\text { events }\end{array}$ & $\%$ obs & $\begin{array}{c}\# \\
\text { even } \\
\text { ts }\end{array}$ & $\%$ obs & $\begin{array}{c}\# \\
\text { event } \\
\text { s } \\
\end{array}$ & $\%$ obs \\
\hline & & \multicolumn{4}{|c|}{ TRANSITION } & \multicolumn{4}{|c|}{ BREAKDOWN } \\
\hline Bernhard et al. 2005 & $1913-2005$ & NA & NA & NA & NA & 63 & $1.73 \%$ & 54 & $1.69 \%$ \\
\hline Boix et al. 2013 & $1801-2007$ & 134 & $0.83 \%$ & 108 & $1.23 \%$ & 83 & $0.52 \%$ & 65 & $0.74 \%$ \\
\hline Cheibub et al. 2010 & $1947-2008$ & 102 & $1.14 \%$ & 102 & $1.14 \%$ & 66 & $0.74 \%$ & 66 & $0.74 \%$ \\
\hline Freedom House (FIW: free) & $1973-2015$ & 91 & $1.19 \%$ & 91 & $1.19 \%$ & 72 & $0.94 \%$ & 72 & $0.94 \%$ \\
\hline Geddes et al. 2014 & $1946-2009$ & 103 & $1.32 \%$ & 103 & $1.32 \%$ & 75 & $0.96 \%$ & 75 & $0.96 \%$ \\
\hline Magaloni et al. 2013 & $1951-2012$ & 83 & $0.98 \%$ & 83 & $0.98 \%$ & 49 & $0.58 \%$ & 49 & $0.58 \%$ \\
\hline Polity IV $(A N=A U T)$ & $1801-2014$ & 131 & $0.80 \%$ & 106 & $1.17 \%$ & 80 & $0.49 \%$ & 64 & $0.71 \%$ \\
\hline Reich 2002 & $1801-1998$ & 131 & $1.22 \%$ & 106 & $1.79 \%$ & 92 & $0.86 \%$ & 73 & $1.23 \%$ \\
\hline Svolik 2012 & $1946-2007$ & 89 & $1.07 \%$ & 89 & $1.07 \%$ & 57 & $0.69 \%$ & 57 & $0.69 \%$ \\
\hline Ulfelder 2012 & $1956-2010$ & 113 & $1.51 \%$ & 113 & $1.51 \%$ & 109 & $1.45 \%$ & 109 & $1.45 \%$ \\
\hline \multirow[t]{2}{*}{ Wabman et al. 2013} & $1973-2010$ & 98 & $1.51 \%$ & 98 & $1.51 \%$ & 62 & $0.95 \%$ & 62 & $0.95 \%$ \\
\hline & & \multicolumn{4}{|c|}{ LIBERALIZATION } & \multicolumn{4}{|c|}{ BACKSLIDING } \\
\hline $\begin{array}{l}\text { Freedom House (FIW, any } \\
\text { change) }\end{array}$ & $1973-2015$ & 919 & $12.04 \%$ & 919 & $12.04 \%$ & 738 & $9.67 \%$ & 738 & $9.67 \%$ \\
\hline Polity IV (any change) & $1801-2014$ & 811 & $4.94 \%$ & 556 & $6.13 \%$ & 511 & $3.11 \%$ & 341 & $3.76 \%$ \\
\hline Skaaning et al. 2015 & $1801-2015$ & 657 & $3.80 \%$ & 379 & $3.66 \%$ & 483 & $2.79 \%$ & 261 & $2.52 \%$ \\
\hline$V$-Dem: Electoral Democracy & $1901-2015$ & 803 & $5.05 \%$ & 669 & $6.50 \%$ & 400 & 2.52 & 302 & $2.93 \%$ \\
\hline $\begin{array}{l}\text { Wabman et al. } 2013 \text { (any } \\
\text { change) }\end{array}$ & $1973-2010$ & 1033 & $15.87 \%$ & 1033 & $15.87 \%$ & 730 & $11.21 \%$ & 730 & $11.21 \%$ \\
\hline
\end{tabular}

\section{Agreement between Measures of Regime Change}

It is commonly assumed that measures of regime change are interchangeable because the indicators or regime type that they are based on correlate very highly. Table 2 refutes this conventional wisdom. It presents the average pairwise correlation coefficients for all indicators of rupture and reform and contrasts them with the average correlation among the corresponding democracy indicators. ${ }^{8}$ While it is indeed the case that the average pairwise correlation between the regime type indicators is very high (between 0.85 and 0.86), we find strikingly low correlations among all indicators of regime change. In fact, the correlation ranges between a high 0.46 (transition) and a low 0.33 (backsliding).

This turns our attention to the study of agreement between the various indicators, which is defined as the number of indicators coding the respective regime change event, divided by the total number of indicators available. Thus, agreement ranges from 0 to 1 . All cases coded as 0 are excluded from the analysis because they have plausibly not experienced the respective form of regime change. ${ }^{9}$

\footnotetext{
${ }^{8}$ For an overview of all pairwise correlations, see Tables A3 through A6 in Appendix II.

${ }_{9}^{9}$ All analyses were carried out with an alternative coding of agreement that looks at a period of three years $(t+/-1)$. This is a relatively generous coding because it codes agreement between indicators even when the change was
} 
Table 2: Average Correlations among Indicators of Regime Change

\begin{tabular}{|l|ccc|}
\hline & Democracy & Transition & Breakdown \\
\hline Bernhard et al. 2005 & NA & NA & 0.56 \\
Boix et al. 2013 & 0.86 & 0.49 & 0.47 \\
Cheibub et al. 2010 & 0.85 & 0.50 & 0.51 \\
Freedom House (FIW: free) & 0.79 & 0.31 & 0.22 \\
Geddes et al. 2014 & 0.87 & 0.49 & 0.48 \\
Magaloni et al. 2013 & 0.87 & 0.53 & 0.50 \\
Polity IV (AN=AUT) & 0.83 & 0.40 & 0.39 \\
Reich 2002 & 0.80 & 0.39 & 0.49 \\
Svolik. 2012 & 0.88 & 0.56 & 0.50 \\
Ulfelder 2012 & 0.85 & 0.53 & 0.49 \\
Wahman et al. 2013 & 0.86 & 0.40 & 0.23 \\
& $0.85(0.03)$ & $0.46(0.08)$ & $0.44(0.11)$ \\
\hline \multicolumn{1}{|c|}{ Mean (SD) } & Democracy & Liberalization & Backsliding \\
\hline Freedom House (FIW, any change) & 0.86 & 0.45 & 0.39 \\
Polity IV (any change) & 0.85 & 0.37 & 0.33 \\
Skaaning et al. 2015 & 0.86 & 0.32 & 0.26 \\
V-Dem: Electoral Democracy & 0.85 & 0.34 & 0.23 \\
Wahman et al. 2013 (any change) & 0.89 & 0.52 & 0.43 \\
\multicolumn{1}{|c|}{ Mean (SD) } & $0.86(0.02)$ & $0.40(0.08)$ & $0.33(0.08)$ \\
\hline
\end{tabular}

We measure agreement for each form of regime change as well as for backsliding and breakdown, and for liberalization and transition. Figure 1 depicts agreement rates over time, averaged over a period of five years. ${ }^{10}$ It underscores that despite the considerable overlap in underlying data sources, average agreement is disturbingly low. On average, agreement between the rupture measures is slightly less than 20 percent, and roughly 40 to 50 percent for reform.

Furthermore, only a handful of cases are unequivocally identified as regime change events by all indicators available. We find 100 percent agreement at time t for 145 backsliding events (out of a total of 1,699; equaling 8.53 percent), 20 instances of breakdown (out of 352; 5.68 percent), 357 liberalizations (out of 2,335; 15.29 percent), and 14 transitions (out of 457; 3.06 percent). Agreement is no more than 50 percent in 76.87 percent of the backsliding, 80.11 percent of the breakdown, 66.77 percent of the liberalization, and 80.74 percent of the transition cases.

recorded in one source a full two years before another indicator picked it up. All results reported here remain robust to this more generous measure (see Appendix).

${ }^{10}$ See Appendix for similar figures on agreement by year, using the extended set of indicators, and expanding the window to a three-year period. 


\section{Figure 1: Average Agreement}
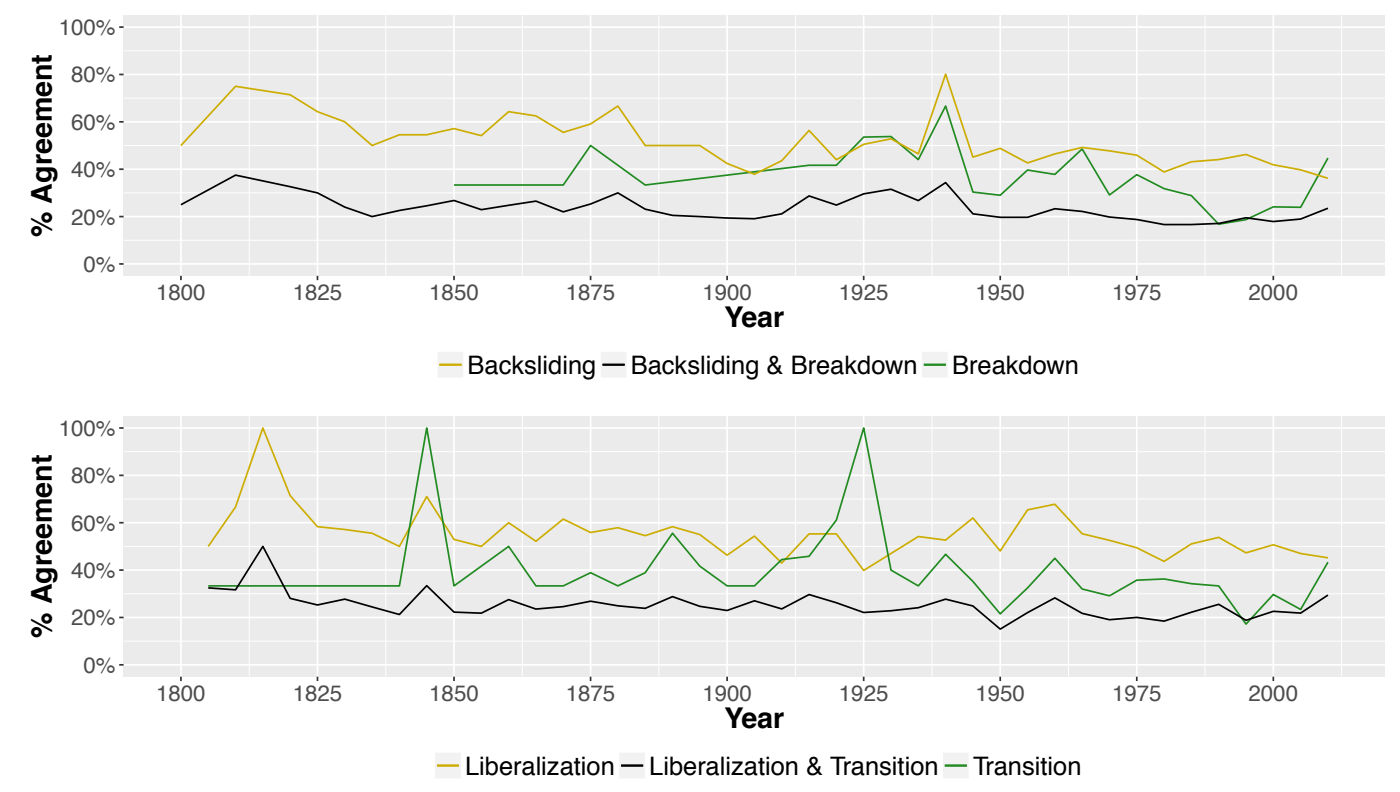

\section{Explaining Variation in Agreement}

What explains variation in agreement across regime change events? We argue that focal pointsthat is, the presence of elections in the case of transition and liberalization, or coups in the case of breakdown and backsliding_-play an important role in explaining agreement.

To understand why focal points have a significant impact on the agreement of indicators, we need to recognize the crucial role of coders in producing regime type ratings (Bollen and Paxton 2000). Usually, the majority of country-years are coded retrospectively, and information about specific countries becomes sparser as we move back in time. As information becomes less available, coders increasingly build their ratings around notable political events such as coups or elections that unequivocally characterize political changes and thus facilitate the identification of regime change events and justify a particular rating.

Additionally, coding rules often explicitly call for coups or elections to precede a change in a country's rating. For example, Gasiorowski (1996: 472) admits that gradual change is possible, but his rating changes mostly in the aftermath of an election or a coup. We find a similar focus on focal points in the coding rules of the Freedom in the World data:

"A score is typically changed only (italics authors) if there has been a real-world development during the year that warrants a decline or improvement (e.g., a crackdown on the media, the country's first free and fair elections), though gradual changes in conditions, in the absence of a signal event, are occasionally registered in the scores" (Freedom House 2016). 
Naturally, regime changes do not exclusively take place in the context of "signal events." Indeed, there is reason to believe that regime change in terms of fewer or greater restrictions on civil and political liberties may take place long before elections are held or coups are staged. But elections or coups provide focal points around which researchers and policymakers may become aware of changing democratic practices, facilitating the identification of ruptures or reforms. Therefore, we expect agreement between different indicators of rupture or reform to be higher for regime change cases that coincide with focal points—and in particular elections and coups.

Importantly, finding positive effects of coups and elections on agreement does not suggest that coups and elections increase the probability of rupture or reform, but rather they increase the likelihood that multiple indicators code a specific regime change event in the same year. This would suggest that regime changes accompanied by such events are more likely to be picked up than those that are not. This, in turn, would imply that we can only identify with certainty a-plausibly unrepresentative — set of actual regime change events.

We test this argument using data from the National Elections Across Democracy and Autocracy (NELDA) dataset (Hyde and Marinov 2012). We rely on the dataset's sample to code years with and without Election. ${ }^{11}$ Our Coup variable codes country-years that experienced at least one successful (military or civilian) coup in year $t$ (data from Powell and Thyne 2011). Our agreement measures for the various forms of regime change are defined as above. Due to data availability, the following analysis is restricted to the period 1950-2010. The universe of cases examined here is all those country-years for which at least one indicator coded a regime change.

Table 3 depicts average agreement across the backsliding, breakdown, liberalization, and transition measures. It confirms previous findings and shows that agreement is generally higher for reform (44 to 52 percent) than for rupture ( 28 to 30 percent). More importantly, it points to strong differences in agreement rates between backsliding and breakdown cases that did and did not experience coups. Coups are associated with an increase in agreement by up to 33 percentage points (breakdown). In contrast, they are associated with marginal increases in agreement among the liberalization and transition indicators only. The reverse is true for elections, which increase agreement of the liberalization and transition measures by up to 17 percentage points (transition), but affect agreement on changes toward greater autocratic qualities only marginally.

\footnotetext{
${ }^{11}$ Note that some microstates as well as countries without national elections (e.g., China, Saudi Arabia, United Arab Emirates) were excluded from NELDA and thus are missing in our dataset.
} 
Table 3: Average agreement around focal points, 1950-2010

\begin{tabular}{|l|ccccc|}
\hline & Mean & No coups & Coups & $\begin{array}{c}\text { No } \\
\text { Elections }\end{array}$ & Elections \\
\hline Breakdown & $28.28 \%$ & $17.53 \%$ & $50.47 \%$ & $29.05 \%$ & $25.68 \%$ \\
Backsliding & $43.92 \%$ & $41.00 \%$ & $57.49 \%$ & $43.04 \%$ & $43.78 \%$ \\
Backsliding \& & & & & & \\
Breakdown & $18.82 \%$ & $15.67 \%$ & $33.64 \%$ & $17.87 \%$ & $19.96 \%$ \\
\hline Transition & $29.87 \%$ & $29.32 \%$ & $35.53 \%$ & $17.94 \%$ & $35.01 \%$ \\
Liberalization & $51.48 \%$ & $51.43 \%$ & $52.16 \%$ & $42.94 \%$ & $55.96 \%$ \\
Liberalization \& & & & & & \\
Transition & $21.73 \%$ & $21.53 \%$ & $24.49 \%$ & $16.51 \%$ & $27.27 \%$ \\
\hline
\end{tabular}

We employ OLS regression analysis to test this argument further, regressing agreement on the coup and election dummy variables. Apart from these focal points, other factors may contribute to average agreement rates. Agreement may be affected by the importance of a country in the world community; larger or politically and economically more powerful countries are under increased international scrutiny, affecting the attention paid by researchers and nongovernmental organizations. This may also increase the availability of information about these countries. Consequently, we employ GDP, Population size, and GDP per capita as control variables (source: World Bank 2015). All three measures are logged. We further add Trade openness (sum of exports and imports divided by GDP; World Bank 2015) and GATT/WTO membership (source: Ulfelder 2012). Moreover, more aid-dependent countries may be subject to more international scrutiny. We use log Aid per capita (source: World Bank 2015). Finally, we control for the Number of measures available (log) for the respective form of regime change examined; agreement may be lower if more measures are available. Elections and Coups are measured in t, membership in GATT/WTO is measured in $\mathrm{t}-1$, and the remainder is averaged across the three years preceding the year of analysis. All models include country- and time-fixed effects. Standard errors are clustered by country.

Table 4 summarizes our findings. We examine the effect of coups and elections on agreement among the breakdown (Model 1), backsliding (Model 2), breakdown and backsliding (Model 3), transition (Model 4), liberalization (Model 5), and transition and liberalization indicators (Model 6).

Overall, the results suggest that agreement of the breakdown and backsliding indicators is statistically significantly higher around coups, while elections increase agreement rates of the transition and liberalization measures. Successful coups increase agreement for breakdown (backsliding) by about 27.6 (18.9) percentage points, while elections are associated with an increase in agreement for transition (liberalization) by 14.1 (16.5) percentage points. All these estimates are statistically significant at the 1\% level. Elections are generally not statistically 
significantly related to agreement of the backsliding and breakdown measures, while coups lack predictive power with respect to the agreement between transition and liberalization indicators. ${ }^{12}$ Coups and elections increase agreement between indicators of backsliding and breakdown, while elections increase agreement of the liberalization and transition measures, all else equal. Yet, coups and elections are closely related to agreement over indicators, but they are not determinative of regime change. These focal events shape societal expectations of change, but as Schedler (2001) notes, "even transitions ignited by focal events often unfold within a larger framework of oscillation or incremental transformation." Moreover, they accompany only a minority of regime changes. Our dataset counts only 402 country-years with at least one successful coup (3.2\% of all country-years for which coup-data are available). Similarly, elections took place in only about 26 percent of our country-year observations.

Table 4: Agreement between regime change indicators

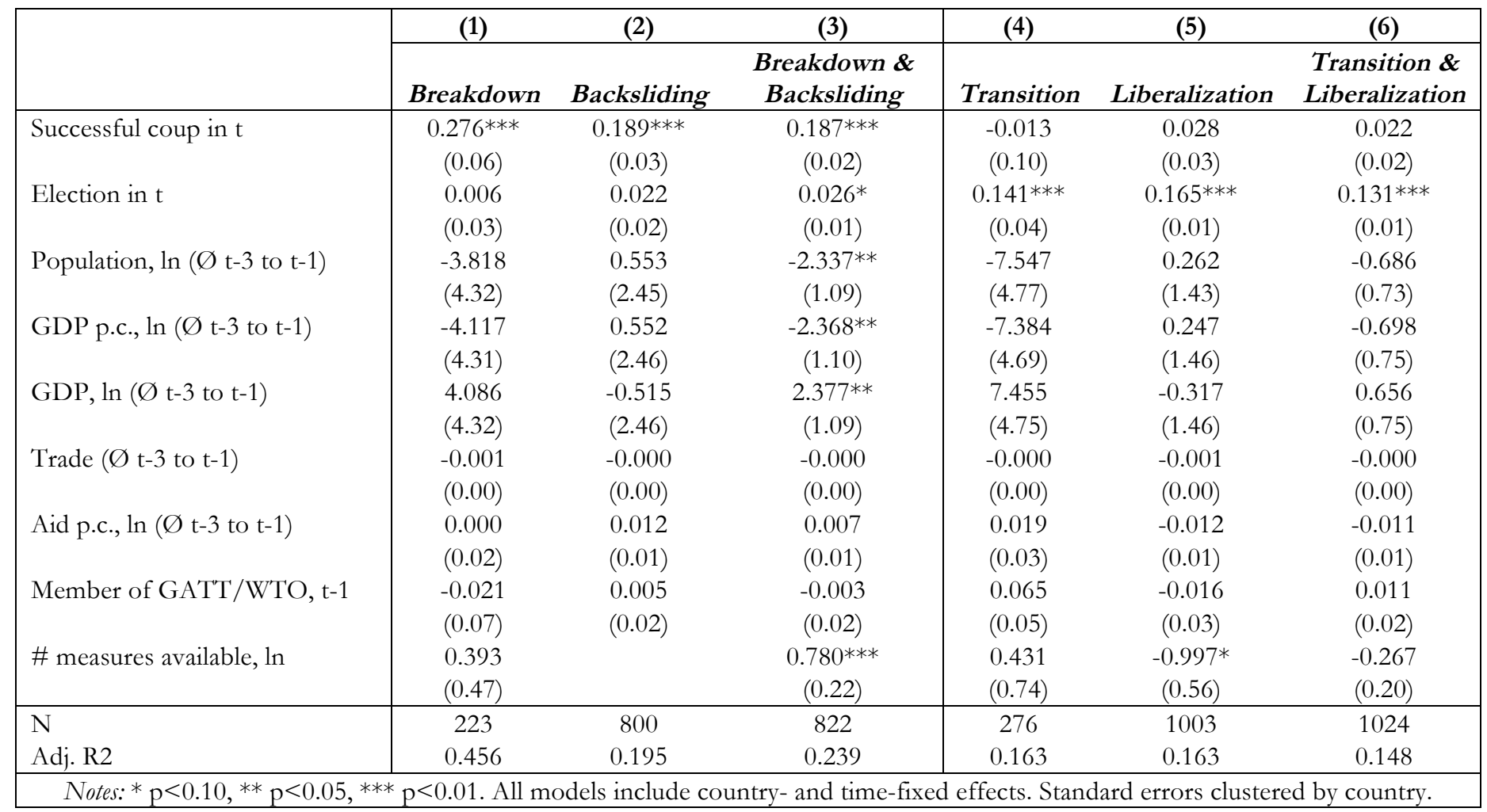

12 Our results remain robust when agreement is measured over a period of three instead of one year (see Appendix). 


\section{Indicators of Regime Change and Accumulation of Knowledge}

The low agreement between indicators of regime change has important ramifications for empirical analysis. Conclusions drawn on one set of dependent variables are often not supported by analyses employing alternative measures of regime change. This draws the validity of studies of regime change into question, and suggests that the lack of cumulative knowledge about regime change is in part due to the ambiguity and unreliability of currently used measurements.

To illustrate the dependence of findings on the specific measure of regime change employed, we examined the robustness of empirical models of rupture and reform to alternative indicators of regime change. The studies considered here investigate the effect of a broad set of independent (i.e., socioeconomic, institutional, international) variables and use different methodological approaches to the study of regime change. They are classified into one of the four types of regime change according to the coding of their dependent variable ${ }^{13}$ :

On liberalization, we include Burke and Leigh's (2010) study on the impact of economic growth on regime changes toward democracy and autocracy. They argue that economic growth reduces the likelihood of both liberalization and backsliding. We further include Cornell (2013), who finds that democracy aid has the most positive effect on the likelihood of liberalization in one-party regimes. Third, we include Ansell and Samuels' (2010) work on the effects of land and income inequality on liberalization. They posit that autocracies with more equal land and less equal income distributions are more likely to democratize.

As to backsliding, we again include Burke and Leigh (2010) again because they study both liberalization and backsliding. Further, we add Gibler and Randazzo (2011), who find that established independent judiciaries reduce the likelihood of democratic backsliding, while newly formed judiciaries have no such effect. Third, we examine the robustness of Goldstone et al.'s (2010) finding that it is political regime types, not socioeconomic variables that explain the onset of adverse political change.

Regarding transition, we add Pevehouse (2002), who posits that membership in international organizations is positively linked to the likelihood of democratization. Next, Donno (2013) examines the effects of pre-electoral opposition coalitions and international conditionality on election outcomes in electoral autocracies and finds that competitive autocracies are more

\footnotetext{
13 We thank all the authors of these studies for kindly sharing their data with us and are grateful for their comments and feedback on our findings and this paper.
} 
likely to democratize after elections. Moreover, both opposition coalitions and international conditionality increase the likelihood of post-electoral transition.

We consider two studies of breakdown. Andersen et al. (2014) argue that state capacity in the form of administrative effectiveness stabilizes democratic rule and thus lowers the risk of breakdown. Finally Houle (2009) contends that inequality has no effect on transition to democracy, but increases the likelihood of democratic breakdown. ${ }^{14}$

We leave the sample definition of the original model unchanged. Yet, as indicators cover different time periods and countries, the number of observations in our models differs at times from the original studies. We never added observations to the analysis, but observations were dropped if they could not experience the respective form of regime change as defined by the dependent variable. ${ }^{15}$ We ran all reform models on both a full set of country-years that can potentially experience the regime change event of interest ${ }^{16}$, and on a sample restricted to democracies (backsliding) or autocracies (liberalization). ${ }^{17}$

Table 5: Testing the robustness of extant studies on rupture using alternative measures of regime change

\begin{tabular}{|c|c|c|c|c|c|c|c|c|c|}
\hline \multirow{3}{*}{ Coding DV } & \multicolumn{5}{|c|}{ TRANSITION } & \multicolumn{4}{|c|}{ BREAKDOWN } \\
\hline & \multirow[t]{2}{*}{ scope } & \multicolumn{2}{|c|}{$\begin{array}{l}\text { Pevehouse } \\
\text { 2002b }\end{array}$} & \multicolumn{2}{|c|}{ Donno 2013} & scope & $\begin{array}{r}\text { Anders } \\
2\end{array}$ & $\begin{array}{l}\text { n et al. } \\
14\end{array}$ & $\begin{array}{l}\text { Houle } \\
2009\end{array}$ \\
\hline & & $T 2 M 1$ & $T 2$ & & $T 1$. & & $T 1$ & $T 1$ & T4. M1 \\
\hline Bernhard et al. 2001 & AUT & NA & $\mathrm{NA}$ & NA & $\mathrm{NA}$ & DEM & & & \\
\hline Boix et al. 2013 & AUT & & & & & DEM & & & \\
\hline Cheibub et al. 2010 & AUT & & & & & DEM & & & \\
\hline Freedom House (FIW: free) & AUT & & & & & DEM & & & \\
\hline Geddes et al. 2014 & AUT & & & & & DEM & & & \\
\hline Magaloni et al. 2013 & AUT & & & & & DEM & & & \\
\hline Politv IV $(A N=A U T)$ & AUT & & & & & DEM & & & \\
\hline Reich 2002 & AUT & & & & & DEM & & & \\
\hline Svolik 2012 & AUT & & & & & DEM & & & \\
\hline Ulfelder 2012 & AUT & & & & & DEM & & & \\
\hline Wabman et al. 2013 & AUT & & & & & DEM & & & \\
\hline & $\begin{array}{c}\% \\
\text { (tortial) }\end{array}$ & $80.0 \%$ & $40.0 \%$ & $\begin{array}{l}0.0 \% \\
(100 \%)\end{array}$ & $\begin{array}{l}0.0 \% \\
(70 \%)\end{array}$ & $\%$ & $72.7 \%$ & $27.3 \%$ & $27.3 \%$ \\
\hline
\end{tabular}

\footnotetext{
${ }^{14}$ Note that estimates dynamic probit models of regime change. We depart from his original model specification, however, and estimate simple probit models of the determinants of breakdown. Thus, strictly speaking, our robustness checks of Houle (2009) are somewhat less than robustness checks.

${ }^{15}$ For example, all country-years scoring -8 and below on the Polity score were dropped if the dependent variable measured backsliding as decrease on Polity by at least 3 points.

${ }^{16}$ That is, for example, a country scoring a 9 on the Polity IV scale was excluded when the three-point increase on the scale indicates.

${ }^{17}$ We use the indicators' own thresholds of democracy / autocracy, as employed in the analysis of rupture. As neither the Lexical Index of Democracy (Skaaning et al. 2015) nor the V-Dem data (Coppedge et al. 2016a) suggest thresholds to distinguish between democracies and autocracies, these indicators are excluded in this second part of the reform robustness checks.
} 
Table 6: Testing the robustness of extant studies on reform using alternative measures of regime change

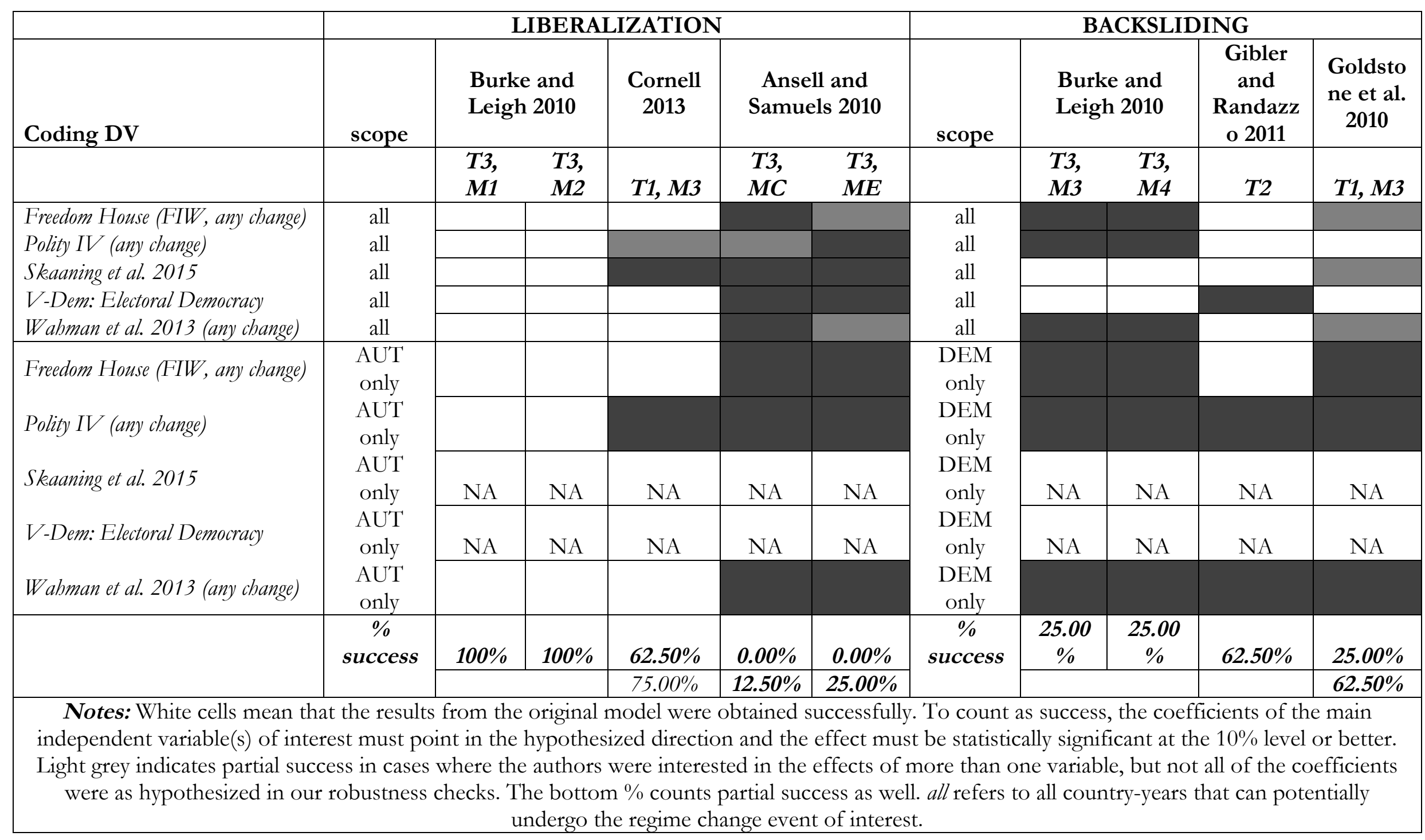


The robustness checks employ all of the indicators identified previously, even though some of the papers analyzed (e.g., Goldstone et al. 2010) restrict their theoretical predictions to major regime changes only. We do so in order to examine the interchangeability of as broad a set of indicators as possible. Further, by ex ante excluding certain indicators, we would have been required to make theoretical assumptions about the causal mechanism at work, which is not the purpose of this study. As a consequence, a disregard of scope conditions may explain some of our results. However, our goal is not to draw into question extant findings, but rather to examine the validity and interchangeability of extant measurements of regime change. Thus, finding that only a few of the robustness checks yielded the same results should not be understood as invalidating the original study's conclusions.

Tables 5 and 6 summarize our results. White cells denote that we obtained the same results as in the original model using the specified dependent variable and scope condition. Dark gray represents failure to do so. Light gray means that we obtained similar results not for all but at least one of the coefficients of interest. To count as success, the coefficient(s) of interest must point in the expected direction and must be statistically significant at the $10 \%$ level or above.

Our results suggest that published findings in the study of regime change depend to a large extent on indicator choice. For the majority of models analyzed here, we failed to obtain the same results for more than 50 percent of the indicators. Success rates are even lower when the extended set of indicators is used (See Appendix). This shows that indicators commonly assumed to tap into similar concepts are generally not interchangeable, and published findings appear to depend on the indicator chosen. Taken together, the robustness checks suggest that we know very little about the factors driving regime change.

\section{Improving Research on Political Regime Change}

Examining measurements of regime change reveals a number of problems. Agreement between them is extremely low, which suggests that they measure different phenomena and that results depend on the choice of indicator. This leads to the conclusion that the lack of accumulated knowledge in the study of regime change is due, at least in part, to imprecise measurements. Unless we develop more comprehensive and reliable measurements of regime change, it is hard to draw empirically sound and valid conclusions about the determinants of regime change and the consequences thereof.

The preceding discussion suggests a number of guidelines and desiderata for future, more rigorous research on regime change across subfields in political science. First is to develop 
a clear consensus over how to conceptualize regime change. Currently, there is a lack of agreement on the definitions of backsliding, breakdown, liberalization, and transition, as well as the underlying characterization of democracy. A distinction between rupture and reform is useful as it is likely that rupture and reform have different causes and plausibly different consequences.

Second is to employ indicators that clearly map onto these regime changes. In our review of the literature, we rarely found that scholars justified their indicator choices. Often, it seems that researchers assume interchangeability between indicators. It is true that the correlation between the various indicators of regime type is high. Yet, this does not translate into a high correlation between the indicators of regime change. Indicators of regime change are not interchangeable, and the choice of indicator is consequential for the empirical analysis, as this paper shows. This calls researchers to be conscious of the choices they make when picking indicators, and consumers of the literature to take into account the different measurement choices when considering lessons learned and adjudicating between findings.

Third is to go beyond conventionally used datasets in the country-year format. Rather, a regime change dataset should code regime change events instead of having one observation for each country and year. The latter may lead scholars to mistakenly treat one long period of regime change as several distinct and shorter regime change events. Consider, for example, V-Dem's indicator of Electoral Democracy in Mexico and Zimbabwe (Figure 2). A standard country-year format masks important regime change patterns in both countries: we identify regime change events in Mexico in 1918, 2000, and 2013, and in Zimbabwe in 1923 and 1978. However, our coding scheme is unable to pick up the gradual backsliding process in Zimbabwe between the end of World War II and the country's formal independence in 1980. Any annual change in electoral democracy was too small to be coded as regime change event, yet the accumulated change was substantial. Similarly, the conventional coding scheme does not tell us when the Mexican liberalization process was initiated. A country-year-based scheme would suggest that liberalization happened between 1999 and 2000. However, as Figure 2 underscores, Mexico had embarked on a gradual liberalization process already around 1970, which accelerated markedly in the late-1980s.

Moreover, no extant measure qualitatively identifies both rupture and reform. Consequently, the creation of an indicator of regime change that picks up both regime rupture and reform, but does not impose arbitrary thresholds between different forms of political rule, is an important task for future researchers. Such an indicator would not only greatly improve research, but may also be used to identify early warning signs of an ensuing large-scale rupture. 
Figure 2: Identifying Years of Regime Change

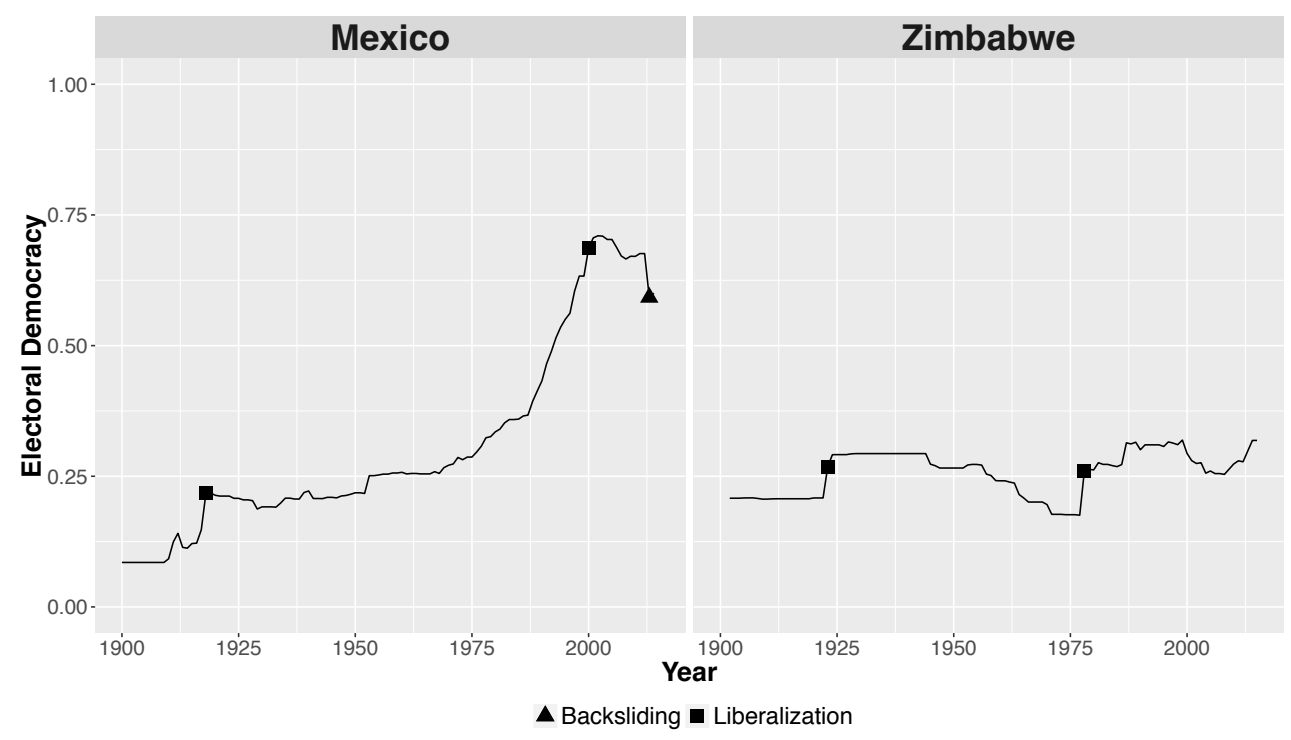

\section{Conclusion}

Despite centuries of thinking, too little is known about the factors driving transition, breakdown, liberalization, and backsliding. Debates over the role of economic conditions, political institutions, cultural characteristics, and other potentially facilitating factors can only be resolved when scholars consciously consider the measures they use and seek to develop research methods that promote cumulative knowledge building. So, too, questions over sequencing and the relationship between rupture and reform can only be answered when these changes are defined carefully and analyses match them. Recognizing the problems with extant measures is the first step in spurring the community to address existing problems, allowing us to engage in a collective conversation over these critical issues.

Moreover, a lack of attention to the conceptualization and measurement of regime change hampers the accumulation of knowledge in other areas of research, such as international security, international political economy, and comparative politics more broadly: does democratization increase trade openness (e.g., Milner and Kubota 2005; Milner and Mukherjee 2009)? Is democratization associated with increased inflows of Foreign Direct Investment (e.g., Li 2009; Pandya 2014)? Are democratized countries less likely to fight one another (e.g., Maoz and Russett 1993; Ward and Gleditsch 1998; Sunde and Cervellati 2013)?

The findings in this paper demonstrate that regime type and regime change indicators are heavily influenced by focal points, which explains some of the variation in agreement between 
regime change indicators. It may well be plausible that most rupture and reform events are preceded by media crackdowns, or visible improvements in the degree of freedom. Yet, because the indicators are so influenced by the easily detected focal points, and the analyses that draw on these indicators are so as well, we are ill-positioned to pick up gradual reforms below the intensity of "signal events," or for our analyses to shed light on such change. This may make scholars and the international community blind for early warning signs of beginning processes of backsliding, and it hinders our ability to study carefully the factors that spur such changes. Ultimately, this delays learning necessary to guide appropriate, early policy responses.

This is especially crucial in the case of backsliding and breakdown. The inability of the international community to recognize such events, and for the scholarly community to help pinpoint factors that make them more likely, potentially grants national leaders more leeway in cutting back political or civil rights. The recent cutbacks on the constitutional court's powers and increases in the government control over the media in Poland, as well as similar movements toward greater authoritarian qualities in Hungary, Russia, and Turkey only underscore the need to further discuss fine-grained measurements of reform and backsliding in particular. 


\section{References}

Acemoglu, Daron, and James A. Robinson. 2006. Economic Origins of Dictatorship and Democracy. Cambridge, UK: Cambridge University Press.

Alvarez, Michael, José A. Cheibub, Fernando Limongi and Adam Przeworski. 1996. "Classifying Political Regimes." Studies in Comparative International Development 31(2): 3-36.

Andersen, David, Jørgen Møller, Lasse Lykke Rørbæk and Svend-Erik Skaaning. 2014. "State capacity and political regime stability." Democratization 21(7): 1305-1325.

Ansell, Ben and David Samuels. 2010. "Inequality and Democratization: A Contractarian Approach.” Comparative Political Studies 43(12): 1543-1574.

Bermeo, Nancy. 2016. “On Democratic Backsliding.” Journal of Democracy 27(1): 5-19.

Bernhard, Michael, Timothy Nordstrom, and Christopher Reenock. 2001. "Economic Performance, Institutional Intermediation and Democratic Breakdown.” Journal of Politics 63(3): 775-803.

Bogaards, Matthijs. 2010. "Measures of Democratization: From Degree to Type to War." Political Research Quarterly 63(2): 475-488.

Boix, Carles. 2003. Democracy and Redistribution. Cambridge, UK: Cambridge University Press.

Boix, Carles, Michael K. Miller and Sebastian Rosato. 2013. “A Complete Data Set of Political Regimes, 1800-2007." Comparative Political Studies 46(12): 1523-1554.

Bollen, Kenneth A. and Robert W. Jackman. 1989. "Democracy, Stability, and Dichotomies." American Sociological Review 54(4): 612-621.

Bollen, Kenneth A. and Pamela Paxton. 2000. “Subjective Measures of Liberal Democracy.” Comparative Political Studies 33(1): 58-86.

Burke, Paul J. and Andrew Leigh. 2010.“Do Output Contractions Trigger Democratic Change?” American Economic Journal: Macroenomics 2(4): 124-157.

Cameron, Maxwell A. and Philip Mauceri, eds. 2006. The Peruvian Labyrinth: Polity, Society, Economy. University Park, PA: Penn State University Press.

Casper, Gretchen and Claudiu Tufis. 2003. "Correlation versus Interchangeability: The Limited Robustness of Empirical Findings on Democracy Using Highly Correlated Data Sets.” Political Analysis 11(2): 195-203.

Cheibub, José A. 2007. Presidentialism, Parliamentarism, and Democracy. Cambridge, UK: Cambridge University Press.

Cheibub, José A., Jennifer Gandhi and James R. Vreeland. 2010. “Democracy and Dictatorship Revisited." Public Choice 143(1-2): 67-101. 
Coppedge, Michael, John Gerring, Staffan I. Lindberg, Svend-Erik Skaaning, Jan Teorell, David Altman, Michael Bernhard, M. Steven Fish, Adam Glynn, Allen Hicken, Carl Henrik Knutsen, Kyle Marquardt, Kelly McMann, Farhad Miri, Pamela Paxton, Daniel Pemstein, Jeffrey Staton, Eitan Tzelgov, Yi-ting Wang, and Brigitte Zimmerman. 2016a. "V-Dem [Country-Year/Country-Date] Dataset v6.2." Gothenburg, Sweden: Varieties of Democracy (V-Dem) Project. https://www.v-dem.net/en/data/data-version-6-2/ (retrieved 10/30/2016).

Coppedge, Michael, John Gerring, Staffan I. Lindberg, Svend-Erik Skaaning, Jan Teorell, Frida Andersson, Kyle L. Marquardt, Valeriya Mechkova, Farhad Miri, Daniel Pemstein, Josefine Pernes, Natalia Stepanova, Eitan Tzelgov, and Yi-ting Wang. 2016b. "V-Dem Methodology v6.” Varieties of Democracy (V-Dem) Project. https://www.vdem.net/files/42/Methodology\%20v6.pdf (retrieved 10/30/2016).

Coppedge, Michael, Staffan I. Lindberg, Svend-Erik Skaaning, and Jan Teorell. 2015. "Measuring high level democratic principles using the V-Dem data." Forthcoming in International Political Science Review.

http://ips.sagepub.com/content/early/2015/12/24/0192512115622046.full.pdf+html (retrieved 10/30/2016).

Cornell, Agnes. 2013. "Does regime type matter for the impact of democracy aid on democracy?” Democratization 20(4): 642-667.

Dahl, Robert A. 1971. Polyarchy: Participation and Opposition. New Haven, CT: Yale University Press.

Dahl, Robert A. 1998. On Democracy New Haven, CT: Yale University Press.

Diamond, Larry. 1999. Developing Democracy: Toward Consolidation. Baltimore, MD: Johns Hopkins University Press.

Donno, Daniela. 2013. "Elections and Democratization in Authoritarian Regimes." American Journal of Political Science 57(3): 703-717.

Doorenspleet, Renske. 2000. "Reassessing the Three Waves of Democratization." World Politics 52(3): 384-406.

Freedom House. various years. "Russia Country Report." https://freedomhouse.org/report/freedom-world/freedom-world-2016 (retrieved 06/24/2016).

Freedom House. 2015. Freedom in the World. Washington, DC: Freedom House. https://freedomhouse.org/report/freedom-world/freedom-world-2015 (retrieved 02/04/2016). 
Freedom House. 2016. "Methodology." https://freedomhouse.org/report/freedom-world2012/methodology (retrieved 04/02/2016).

Gasiorowski, Mark J. 1996. “An Overview of the Political Regime Change Dataset.” Comparative Political Studies 29(4): 469-483.

Geddes, Barbara, Joseph Wright and Erica Frantz. 2013. "New Data on Autocratic Breakdown and Regime Transitions." http://sites.psu.edu/dictators/ (retrieved 10/24/2014).

Geddes, Barbara, Joseph Wright and Erica Frantz. 2014. “Autocratic Breakdown and Regime Transitions: A New Data Set.” Perspectives on Politics 12(2): 313-331.

Gibler, Douglas M. and Kirk A. Randazzo. 2011. "Testing the Effects of Independent Judiciaries on the Likelihood of Democratic Backsliding." American Journal of Political Science 55(3): 696-709.

Goldstone, Jack A., Robert Bates, David L. Epstein, Ted R. Gurr, Michael B. Lustik, Monty G. Marshall, Jay Ulfelder and Mark Woodward. 2010. “A Global Model for Forecasting Political Instability." American Journal of Political Science 54(1): 190-208.

Houle, Christian. 2009. "Inequality and Democracy: Why Inequality Harms Consolidation but Does Not Affect Democratization." World Politics 61(4): 589-622.

Huntington, Samuel. 1992. The Third Wave: Democratization in the Late Twentieth Century. Norman, OK: University of Oklahoma Press.

Hyde, Susan D. and Nikolay Marinov. 2012. "Which elections can be lost?” Political Analysis 20(2): 191-210.

Li, Quan. 2009. "Democracy, Autocracy, and Expropriation of Foreign Direct Investment." Comparative Political Studies 42(8): 1098-1127.

Lindberg, Staffan I. 2015. “Ordinal Versions of V-Dem’s Indices: For Classification, Description, Sequencing Analysis and Other Purposes." V-Dem Working Paper 20. Gothenburg, Sweden: Varieties of Democracies Institute. https://www.vdem.net/media/filer public/f5/32/f532113e-b348-43b8-9830-c0b17928c1cf/vdem working paper 2015 20.pdf (retrieved 10/30/2016).

Linz, Juan J. 2000. Totalitarian and Authoritarian Regimes. Boulder, CO: Lynne Rienner Press. Maeda, Ko. 2010. “Two Modes of Democratic Breakdown: A Competing Risks Analysis of Democratic Durability." Journal of Politics 72(4): 1129-1143.

Magaloni, Beatriz, Jonathan Chu and Eric Min. 2013. Autocracies of the World, 1950-2012 (Version 1.0). Dataset. Stanford, CA: Stanford University. http://cddrl.fsi.stanford.edu/research/autocracies_of_the_world_dataset (retrieved 10/30/2014). 
Maoz, Zeev and Bruce Russett. 1993. "Normative and Structural Causes of the Democratic Peace, 1946-1986." American Political Science Review 87(3): 624-638.

Marshall, Monty G., Keith Jaggers and Ted R. Gurr. 2014. Polity IV Annual Time-Series 18002013. http://www.systemicpeace.org/(retrieved 10/24/2014).

Milner, Helen and Keiko Kubota. 2005. "Why the Move to Free Trade? Democracy and Trade Policy in the Developing Countries.” International Organization 59(1): 707-743.

Milner, Helen and Bumba Mukherjee. 2009. "Democratization and Economic Globalization." Annual Review of Political Science 12: 163-181.

Muller, Edward N. 1995. "Economic Determinants of Democracy." American Sociological Review 60(6): 966-982.

Munck, Gerardo L. and Jay Verkuilen. 2002. "Conceptualizing and Measuring Democracy: Evaluating Alternative Indices." Comparative Political Studies 35(1): 5-34.

Pandya, Sonal. 2014. "Democratization and FDI Liberalization, 1970-2000.” International Studies Quarterly 58(3): 475-488.

Paxton, Pamela. 2000. "Women's Suffrage in the Measurement of Democracy: Problems of Operationalization." Studies in Comparative International Development 35(3): 92-111.

Pevehouse, Jon. 2002. "Democracy from the Outside-In? International Organizations and Democratization.” International Organization 56(3): 515-549.

Powell, Jonathan M. and Clayton L. Thyne. 2011. "Global instances of coups from 1950 to 2010: A new dataset." Journal of Peace Research 48(2): 249-259.

Power, Timothy J. and Mark J. Gasiorowski. 1997“'Institutional Design and Democratic Consolidation in the Third World." Comparative Political Studies 30(2): 123-155.

Przeworski, Adam. 1991. Democracy and the Market. Cambridge, UK: Cambridge University Press. Przeworski, Adam, Michael Alvarez, José A. Cheibub and Fernando Limongi. 2000. Democracy and Development: Political Institutions and Material Well-Being in the World, 1950-1990. Cambridge, UK: Cambridge University Press.

Reich, Gary. 2002. "Categorizing Political Regimes: New Data for Old Problems.” Democratization 9(4): 1-24.

Schedler, Andreas. 2001. "Taking Uncertainty Seriously: The Blurred Boundaries of Democratic Transition and Consolidation." Democratization 8(4): 1-22.

Seawright, Jason. 2012. Party-System Collapse: The Roots of Crisis in Peru and Veneruela. Stanford, CA: Stanford University Press.

Skaaning, Svend-Erik, John Gerring and Henrikas Bartusevičius. 2015. “A Lexical Index of Electoral Democracy.” Comparative Political Studies 48(12): 1491-1525. 
Sunde, Uwe and Matteo Cervellati. 2013. "Democratizing for peace? The effect of democratization on civil conflicts." Oxford Economic Papers 66(3): 774-797.

Svolik, Milan. 2012. The Politics of Authoritarian Rule. New York, NY: Cambridge University Press.

Ulfelder, Jay. 2012. Democracy/Autocracy Data Set.

https://dataverse.harvard.edu/dataset.xhtml?persistentId=hdl:1902.1/18836 (retrieved 05/12/2015).

Wahman, Michael, Jan Teorell and Axel Hadenius. 2013. “Authoritarian Regime Types Revisited: Updated Data in Comparative Perspective." Contemporary Politics 18(1): 19-34.

Ward, Michael D. and Kristian S. Gleditsch. 1998. "Democratizing for Peace." American Political Science Review 92(1): 51-61.

World Bank. 2015. World development indicators and global development finance.

http://data.worldbank.org (retrieved 03/14/2015). 


\section{Appendix I: Additional Figures}

Figure A1: Average agreement by year-1-year window

a) core indicators

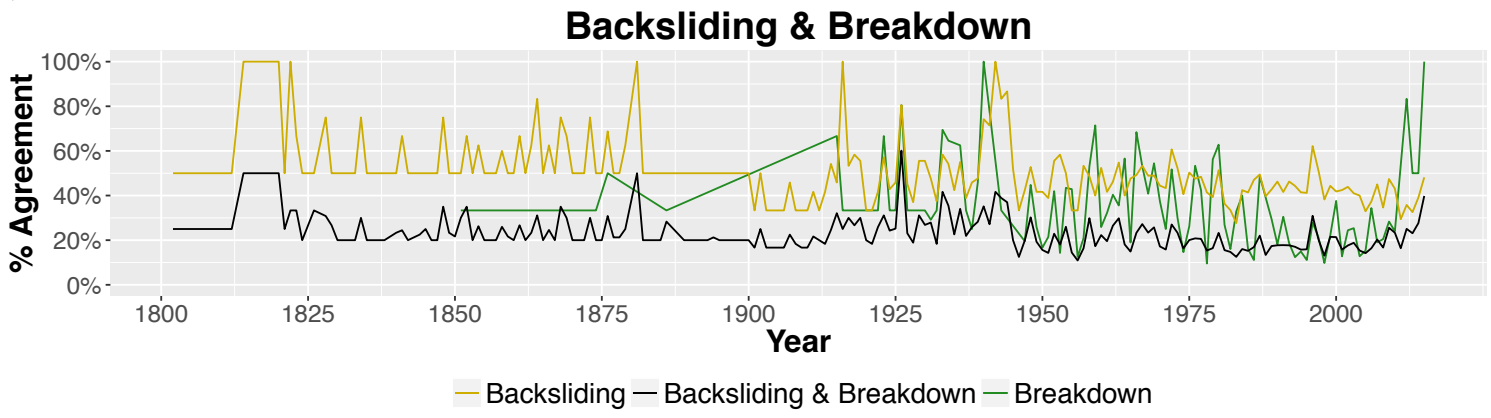

\section{Liberalization \& Transition}

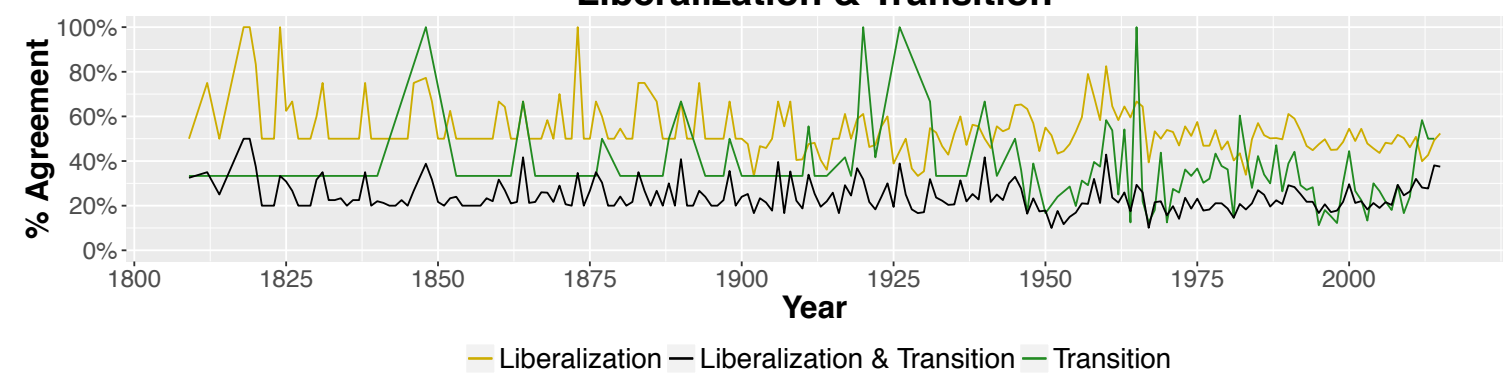


b) extended set of indicators
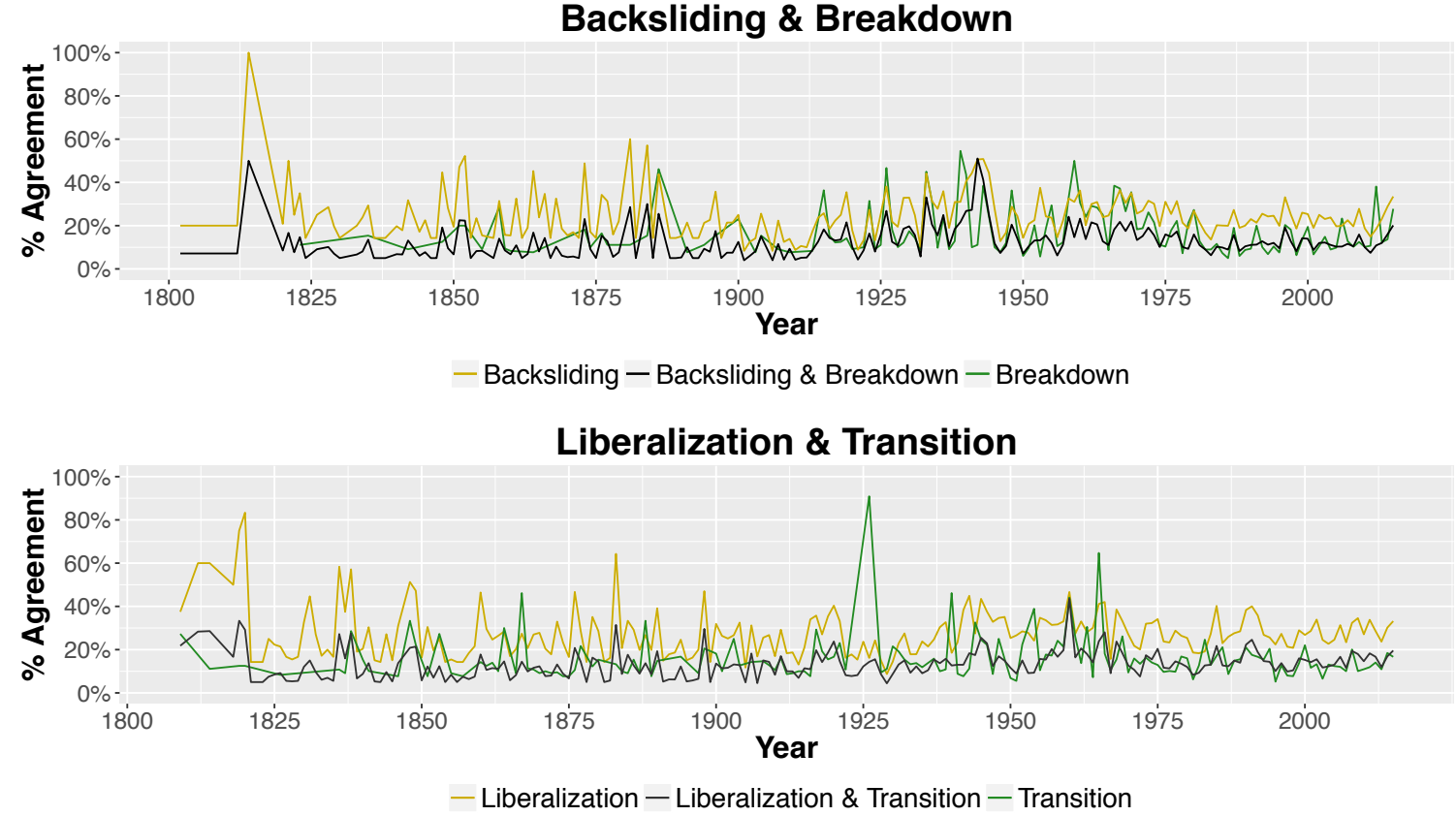

Figure A2: Average agreement by five-year period-1-year window b) extended set of indicators

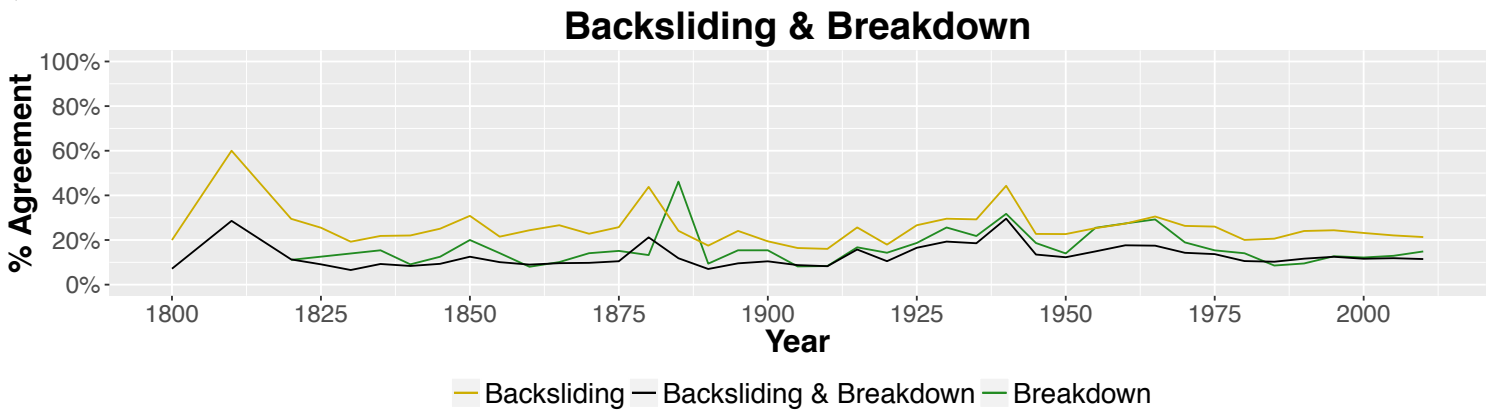

\section{Liberalization \& Transition}

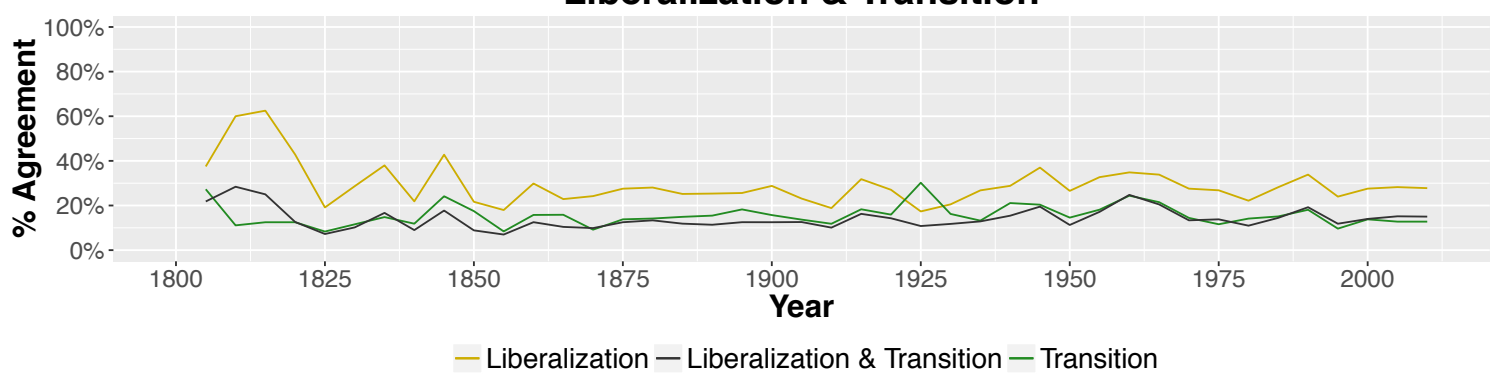


Figure A3: Average agreement by year-3-year window

a) core indicators

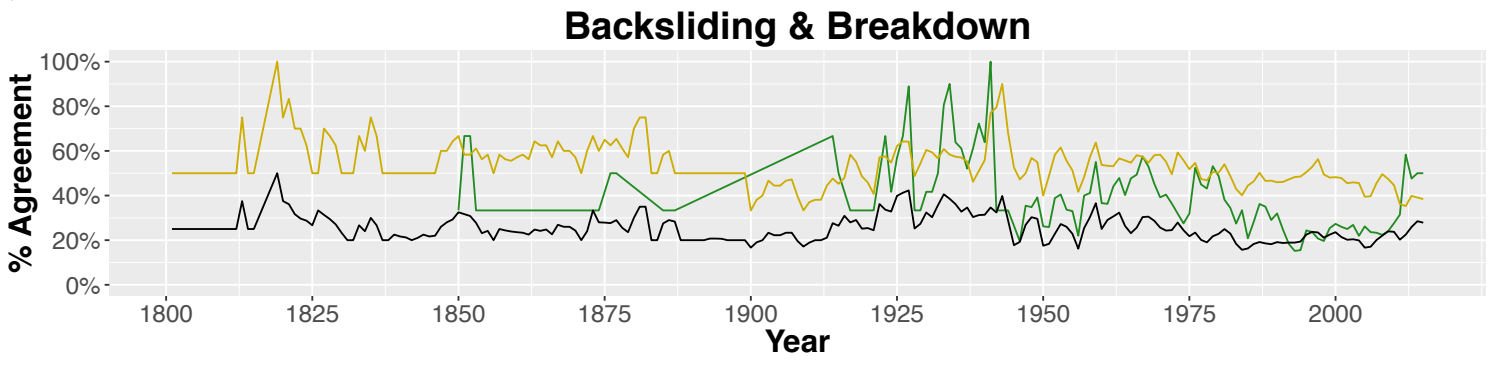

- Backsliding - Backsliding \& Breakdown - Breakdown

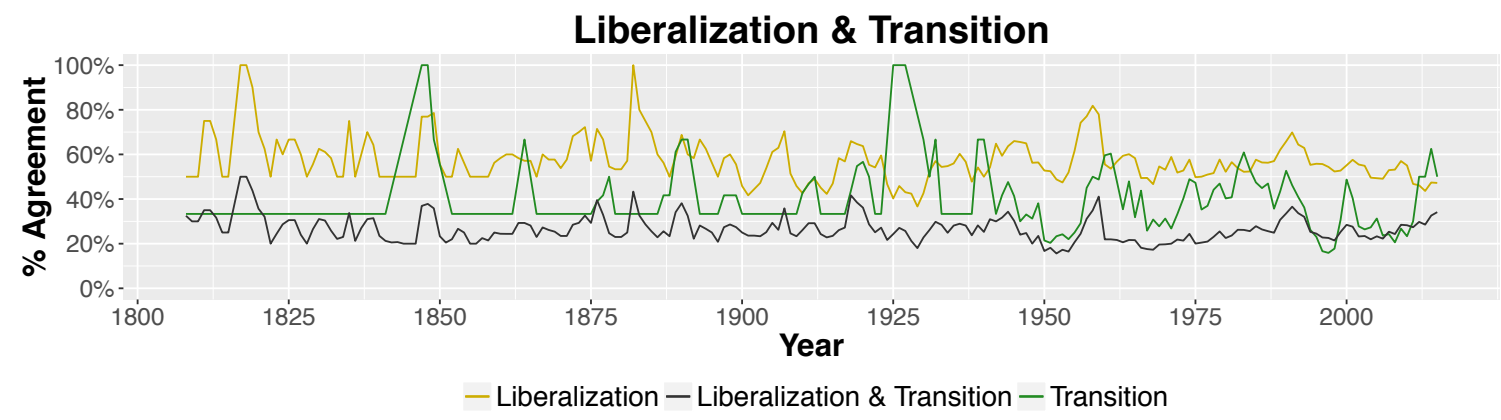

b) extended set of indicators

\section{Backsliding \& Breakdown}

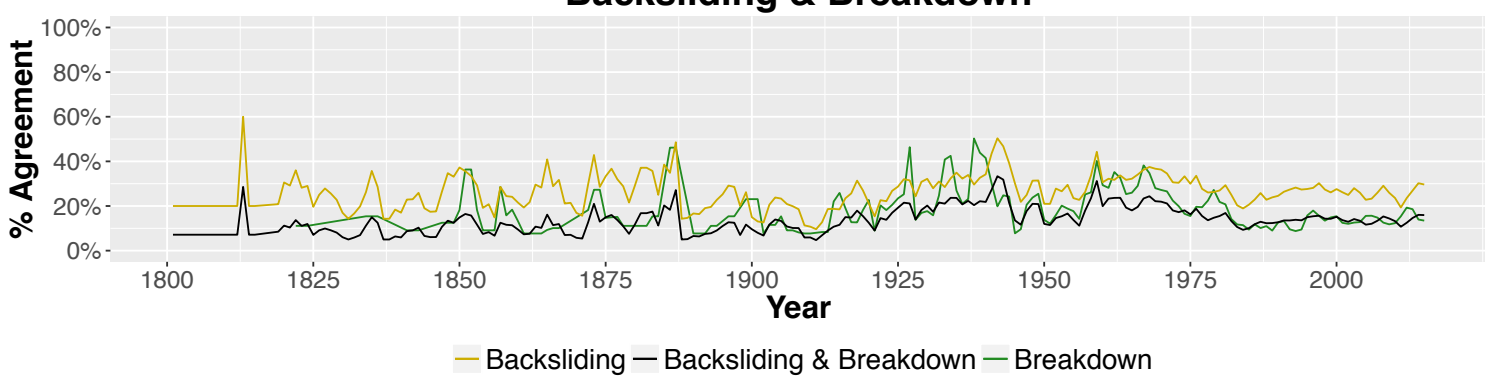

\section{Liberalization \& Transition}

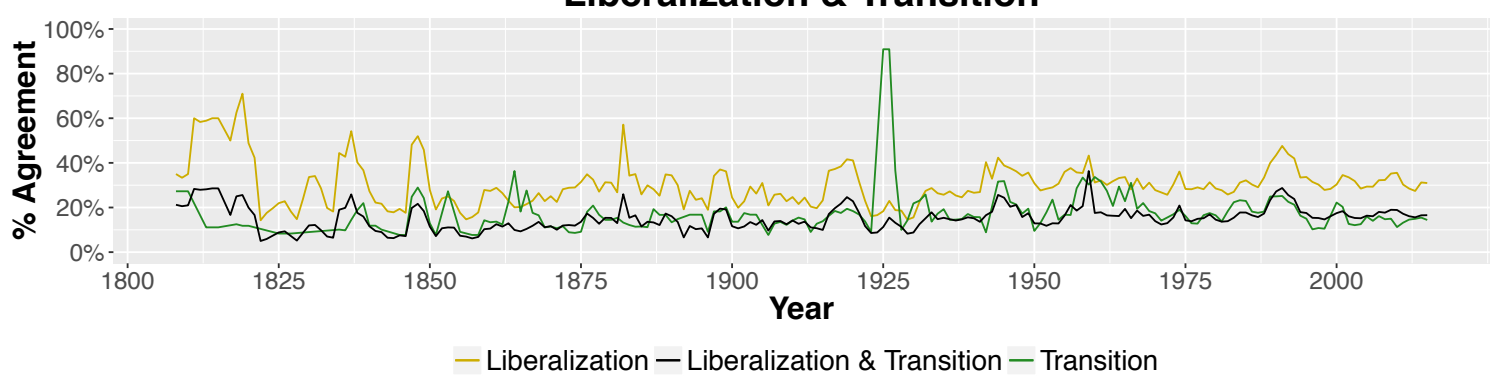


Figure A4: Average agreement by five-year period-3-year window

a) core indicators

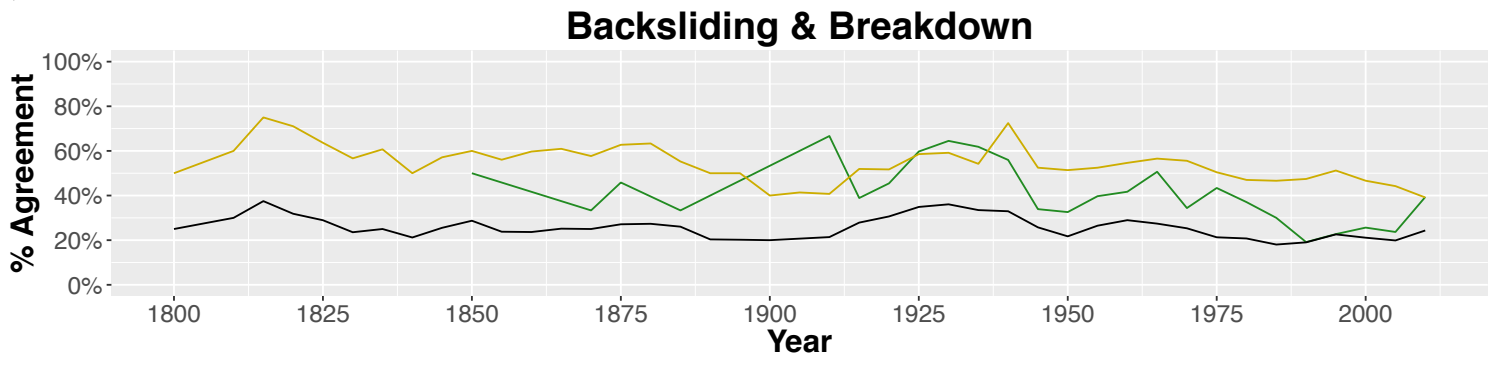

- Backsliding - Backsliding \& Breakdown - Breakdown

\section{Liberalization \& Transition}

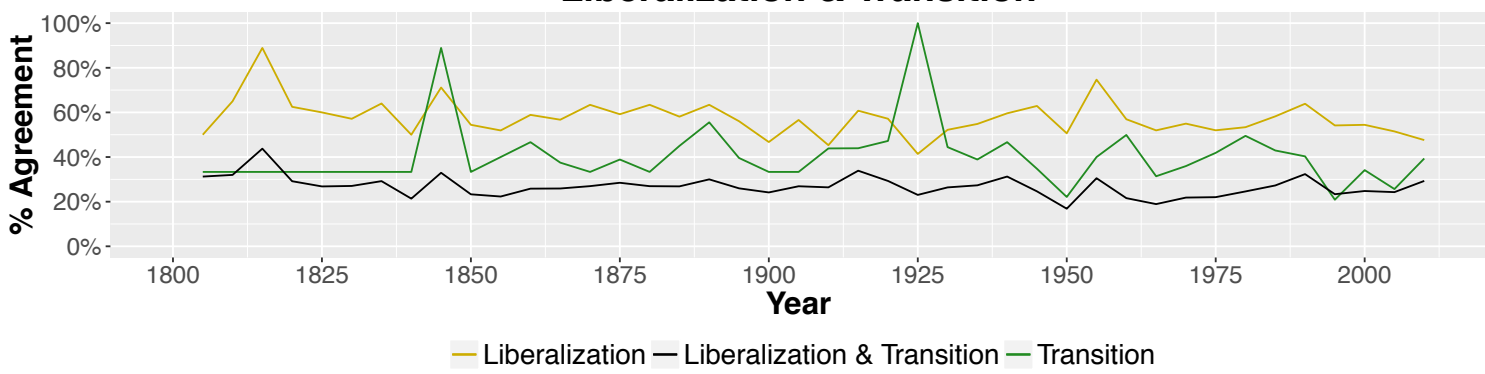

b) extended set of indicators

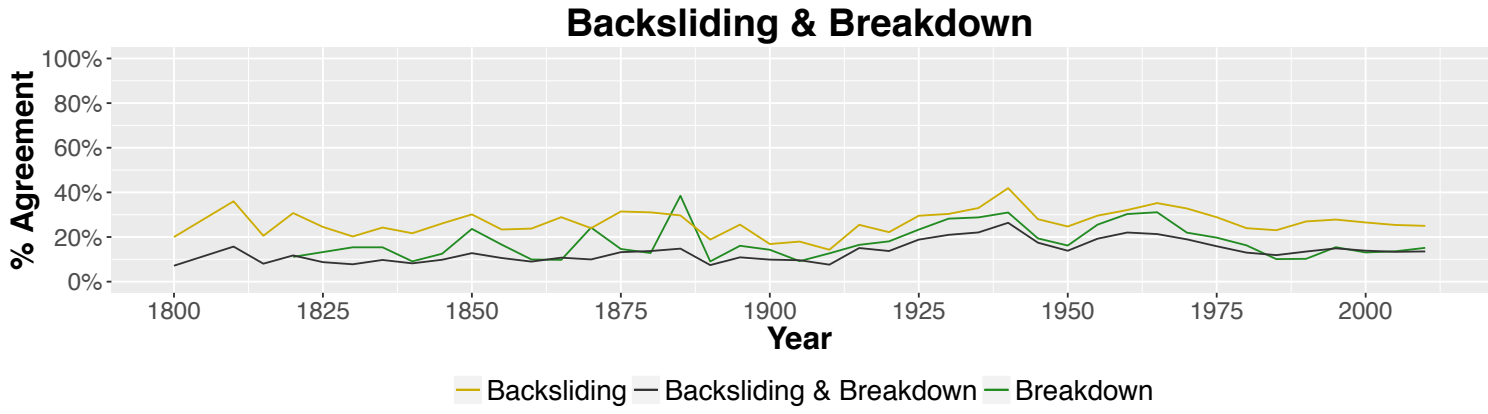

Liberalization \& Transition

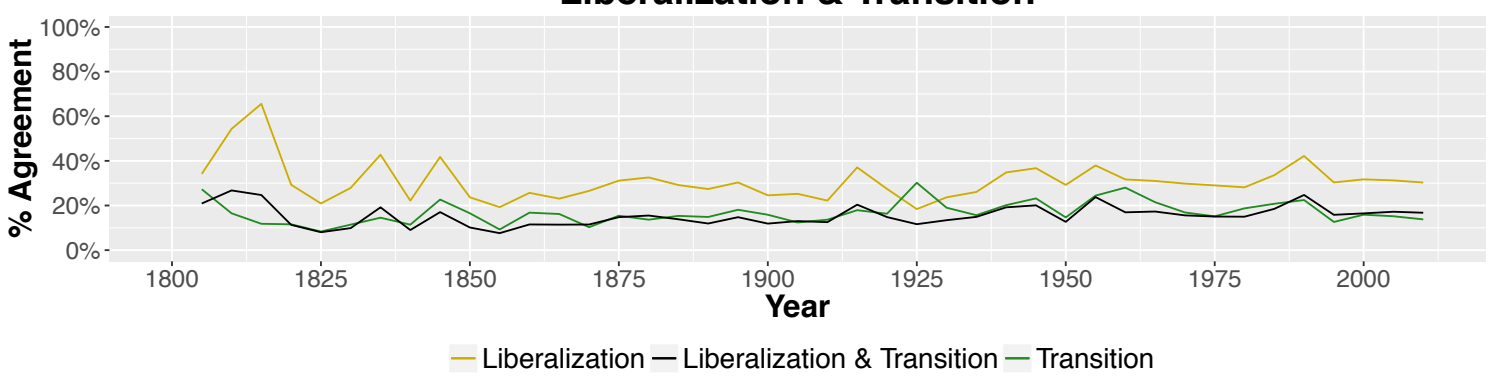




\section{Appendix II: Additional Tables}

Table A1: Indicators of regime change: coding rules and literature examples

\begin{tabular}{|c|c|c|c|c|c|c|c|}
\hline & Indicator name & Definition of regime types & $\begin{array}{l}\text { Definition of } \\
\text { Breakdown/ } \\
\text { Backsliding }\end{array}$ & $\begin{array}{l}\text { Definition of } \\
\text { Transition/ } \\
\text { Liberalization }\end{array}$ & Data source & $\begin{array}{l}\text { Breakdown/ Backsliding } \\
\text { literature examples }\end{array}$ & $\begin{array}{c}\text { Transition/ } \\
\text { Liberalization literature } \\
\text { examples }\end{array}$ \\
\hline & $\begin{array}{l}\text { Bernhard et al. } \\
2005\end{array}$ & authors' coding of backsliding & as defined by authors & NA & $\begin{array}{l}\text { Bernhard et al. } \\
\quad 2005\end{array}$ & $\begin{array}{l}\text { Reenock et al. 2007; Bernhard et } \\
\text { al. 2003; Bernhard et al. } 2004\end{array}$ & \\
\hline & Boix et al. 2013 & authors' coding of DEM and AUT & $\begin{array}{l}\text { regime change from } \\
\text { DEM to AUT }\end{array}$ & $\begin{array}{l}\text { regime change from } \\
\text { AUT to DEM }\end{array}$ & Boix et al. 2013 & $\begin{array}{l}\text { Boix 2003; Svolik 2008; Brambor } \\
\text { and Lindvall } 2014\end{array}$ & Boix 2003 \\
\hline & $\begin{array}{l}\text { Cheibub et al. } \\
2010\end{array}$ & authors' coding of DEM and AUT & $\begin{array}{l}\text { regime change from } \\
\text { DEM to AUT }\end{array}$ & $\begin{array}{l}\text { regime change from } \\
\text { AUT to DEM }\end{array}$ & $\begin{array}{l}\text { Cheibub et al. } \\
2010\end{array}$ & Cheibub 2006 & \\
\hline & $\begin{array}{l}\text { Freedom } \\
\text { House (FIW: } \\
\text { free) }\end{array}$ & $\begin{array}{l}\text { DEM: free; AUT: partly free + not free - FH } \\
\text { Status }\end{array}$ & $\begin{array}{l}\text { regime change from } \\
\text { DEM to AUT }\end{array}$ & $\begin{array}{l}\text { regime change from } \\
\text { AUT to DEM }\end{array}$ & $\begin{array}{l}\text { Freedom House } \\
\quad 2015\end{array}$ & & \\
\hline & $\begin{array}{l}\text { Freedom } \\
\text { House (FIW: } \\
\text { partly free) }\end{array}$ & $\begin{array}{c}\text { DEM: free + partly free; AUT: not free - FH } \\
\text { Status }\end{array}$ & $\begin{array}{l}\text { regime change from } \\
\text { DEM to AUT }\end{array}$ & $\begin{array}{l}\text { regime change from } \\
\text { AUT to DEM }\end{array}$ & $\begin{array}{l}\text { Freedom House } \\
2015\end{array}$ & & \\
\hline & $\begin{array}{l}\text { Freedom } \\
\text { House (PR: } \\
\text { free) }\end{array}$ & $\begin{array}{l}\text { DEM: free; AUT: partly free + not free - } \\
\text { Political Rights only }\end{array}$ & $\begin{array}{l}\text { regime change from } \\
\text { DEM to AUT }\end{array}$ & $\begin{array}{l}\text { regime change from } \\
\text { AUT to DEM }\end{array}$ & $\begin{array}{l}\text { Freedom House } \\
2015\end{array}$ & & \\
\hline 离 & \begin{tabular}{|l|} 
Freedom \\
House (PR: \\
partly free) \\
\end{tabular} & $\begin{array}{l}\text { DEM: free + partly free; AUT: not free - } \\
\text { Political Rights only }\end{array}$ & $\begin{array}{l}\text { regime change from } \\
\text { DEM to AUT }\end{array}$ & $\begin{array}{l}\text { regime change from } \\
\text { AUT to DEM }\end{array}$ & $\begin{array}{l}\text { Freedom House } \\
\quad 2015\end{array}$ & & \\
\hline \multirow[t]{5}{*}{ 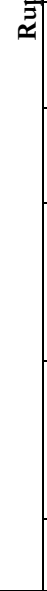 } & $\begin{array}{l}\text { Freedom } \\
\text { House (FIW: } \\
\text { hybrid) }\end{array}$ & $\begin{array}{l}\text { DEM: free; HYB:partly free; AUT: not free - } \\
\text { Status }\end{array}$ & $\begin{array}{l}\text { regime change from } \\
\text { DEM to AUT }\end{array}$ & $\begin{array}{l}\text { regime change from } \\
\text { AUT to DEM }\end{array}$ & $\begin{array}{l}\text { Freedom House } \\
2015\end{array}$ & Starr and Lindborg 2003 & \\
\hline & $\begin{array}{l}\text { Freedom } \\
\text { House (PR: } \\
\text { hybrid) }\end{array}$ & $\begin{array}{c}\text { DEM: } 1 \text { to } 2.5 \text {; HYB: } 3 \text { to 5; AUT: } 5.5 \text { to } 7 \text { - } \\
\text { Political Rights only }\end{array}$ & $\begin{array}{l}\text { regime change from } \\
\text { DEM to AUT }\end{array}$ & $\begin{array}{l}\text { regime change from } \\
\text { AUT to DEM }\end{array}$ & $\begin{array}{l}\text { Freedom House } \\
\quad 2015\end{array}$ & & \\
\hline & $\begin{array}{l}\text { Gasiorowski } \\
1996\end{array}$ & $\begin{array}{l}\text { DEM: democracy and semidemocracy; AUT: } \\
\text { autocracy; regime change with transitions--> } \\
\text { change takes place in the middle of a transition } \\
\text { period; if odd number of transition years: regime } \\
\text { change after }((1 / 2) n+1) / n \text { ) years }\end{array}$ & $\begin{array}{l}\text { regime change from } \\
\text { DEM to AUT }\end{array}$ & $\begin{array}{l}\text { regime change from } \\
\text { AUT to DEM }\end{array}$ & $\begin{array}{l}\text { Gasiorowski } \\
1996\end{array}$ & $\begin{array}{l}\text { Power and Gasiorowski 1997; } \\
\text { Gasiorowski and Power 1998; Lai } \\
\text { and Melkonian-Hoover 2005 }\end{array}$ & $\begin{array}{l}\text { Lai and Melkonian- } \\
\text { Hoover } 2005\end{array}$ \\
\hline & $\begin{array}{l}\text { Gasiorowski } \\
1996 \text { (hybrid) }\end{array}$ & $\begin{array}{l}\text { DEM: democracy; HYB: semidemocracy; AUT: } \\
\text { autocracy; regime change with transitions--- } \\
\text { change takes place in the middle of a transition } \\
\text { period; if odd number of transition years: regime } \\
\text { change after }((1 / 2) \mathrm{n}+1) / \mathrm{n}) \text { years }\end{array}$ & $\begin{array}{l}\text { any change from } \\
\text { DEM to HYB or } \\
\text { HYB to AUT }\end{array}$ & $\begin{array}{l}\text { regime change from } \\
\text { AUT to HYB or HYB } \\
\text { to DEM }\end{array}$ & $\begin{array}{l}\text { Gasiorowski } \\
1996\end{array}$ & & \\
\hline & Gates 2006 & DEM: 0.6 to 1 ; AUT $<0.6$ & $\begin{array}{l}\text { regime change from } \\
\text { DEM to AUT }\end{array}$ & $\begin{array}{l}\text { regime change from } \\
\text { AUT to DEM }\end{array}$ & $\begin{array}{l}\text { Gates et al. } \\
2006\end{array}$ & $\begin{array}{l}\text { Gates et al. 2006; Strand et al. } \\
2013\end{array}$ & $\begin{array}{l}\text { Gates et al. 2006; Strand } \\
\text { et al. } 2013\end{array}$ \\
\hline
\end{tabular}




\begin{tabular}{|c|c|c|c|c|c|c|}
\hline $\begin{array}{l}\text { Geddes et al. } \\
2014\end{array}$ & authors' coding of DEM and AUT & $\begin{array}{l}\text { regime change from } \\
\text { DEM to AUT }\end{array}$ & $\begin{array}{l}\text { regime change from } \\
\text { AUT to DEM }\end{array}$ & $\begin{array}{l}\text { Geddes et al. } \\
2014\end{array}$ & Wright and Escriba-Folch 2012 & \\
\hline $\begin{array}{l}\text { Magaloni et al. } \\
2013\end{array}$ & authors' coding of DEM and AUT & $\begin{array}{l}\text { regime change from } \\
\text { DEM to AUT }\end{array}$ & $\begin{array}{l}\text { regime change from } \\
\text { AUT to DEM }\end{array}$ & $\begin{array}{l}\text { Magaloni et al. } \\
2013\end{array}$ & & \\
\hline $\begin{array}{l}\text { Polity } I V \\
(A N=A U T)\end{array}$ & $\begin{array}{c}\text { DEM: } 6 \text { to } 10 \text {; AUT: } 5 \text { to }-10 \text { (anocracies }= \\
\text { AUT) }\end{array}$ & $\begin{array}{l}\text { regime change from } \\
\text { DEM to AUT }\end{array}$ & $\begin{array}{l}\text { regime change from } \\
\text { AUT to DEM }\end{array}$ & $\begin{array}{l}\text { Marshall et al. } \\
2013\end{array}$ & Maeda 2010 & \\
\hline $\begin{array}{l}\text { Polity } I V \\
(A N=D E M)\end{array}$ & $\begin{array}{c}\text { DEM: } 10 \text { to }-5 \text {; AUT: }-6 \text { to }-10 \text { (anocracies }= \\
\text { DEM) }\end{array}$ & $\begin{array}{l}\text { regime change from } \\
\text { DEM to AUT }\end{array}$ & $\begin{array}{l}\text { regime change from } \\
\text { AUT to DEM }\end{array}$ & $\begin{array}{l}\text { Marshall et al. } \\
2013\end{array}$ & & \\
\hline $\begin{array}{l}\text { Polity IV } \\
\text { (threshold: 7) }\end{array}$ & DEM: 7 to 10 ; AUT: 6 to -10 & $\begin{array}{l}\text { regime change from } \\
\text { DEM to AUT }\end{array}$ & $\begin{array}{l}\text { regime change from } \\
\text { AUT to DEM }\end{array}$ & $\begin{array}{l}\text { Marshall et al. } \\
\quad 2013\end{array}$ & $\begin{array}{l}\text { Lai and Melkonian-Hoover 2005; } \\
\text { Kadera et al. } 2003\end{array}$ & $\begin{array}{l}\text { Epstein et al. 2006; Lai } \\
\text { and Melkonian-Hoover } \\
2005\end{array}$ \\
\hline $\begin{array}{l}\text { Polity IV } \\
\text { (threshold: 1) }\end{array}$ & DEM: 1 to 10 ; AUT: 0 to -10 & $\begin{array}{l}\text { regime change from } \\
\text { DEM to AUT }\end{array}$ & $\begin{array}{l}\text { regime change from } \\
\text { AUT to DEM }\end{array}$ & $\begin{array}{l}\text { Marshall et al. } \\
2013\end{array}$ & Kapstein and Converse 2008 & \\
\hline $\begin{array}{l}\text { Polity IV } \\
\text { (exrec \& } \\
\text { parcomp) } \\
\end{array}$ & $\begin{aligned} \text { DEM: exrec }>= & 6 \text { and parcomp }>=3 \text {; AUT } \\
& \text { otherwise }\end{aligned}$ & $\begin{array}{l}\text { regime change from } \\
\text { DEM to AUT }\end{array}$ & $\begin{array}{l}\text { regime change from } \\
\text { AUT to DEM }\end{array}$ & $\begin{array}{l}\text { Marshall et al. } \\
\quad 2013\end{array}$ & Ulfelder and Lustik 2007 & Ulfelder and Lustik 2007 \\
\hline $\begin{array}{l}\text { Polity IV } \\
\text { (hybrid: } 6 \& \text { - } \\
\text { 6) }\end{array}$ & DEM: 6 to 10 ; HYB: 5 to -5 ; AUT: -5 to -10 & $\begin{array}{l}\text { regime change from } \\
\text { DEM to AUT }\end{array}$ & $\begin{array}{l}\text { regime change from } \\
\text { AUT to DEM }\end{array}$ & $\begin{array}{l}\text { Marshall et al. } \\
\quad 2013\end{array}$ & Pevehouse 2002 & Pevehouse 2002 \\
\hline $\begin{array}{l}\text { Polity IV } \\
\text { (hybrid: } 8 \& 1 \text { ) }\end{array}$ & DEM: 8 to 10 ; HYB: 1 to 7 ; AUT: 0 to -10 & $\begin{array}{l}\text { regime change from } \\
\text { DEM to AUT }\end{array}$ & $\begin{array}{l}\text { regime change from } \\
\text { AUT to DEM }\end{array}$ & $\begin{array}{l}\text { Marshall et al. } \\
2013\end{array}$ & & \\
\hline $\begin{array}{l}\text { Przeworski et } \\
\text { al. } 2000\end{array}$ & authors' coding of DEM and AUT & $\begin{array}{l}\text { regime change from } \\
\text { DEM to AUT }\end{array}$ & $\begin{array}{l}\text { regime change from } \\
\text { AUT to DEM }\end{array}$ & $\begin{array}{l}\text { Przeworski et } \\
\text { al. } 2000\end{array}$ & $\begin{array}{l}\text { Przeworski et al. 2000; Houle } \\
\text { 2009; Shirah 2013; Lai and } \\
\text { Melkonian-Hoover 2005 }\end{array}$ & $\begin{array}{l}\text { Houle 2009; Lai and } \\
\text { Melkonian-Hoover } 2005\end{array}$ \\
\hline Reich 2002 & $\begin{array}{l}\text { DEM: democracy and semidemocracy; AUT: } \\
\text { autocracy; regime change with transitions--> } \\
\text { change takes place in the middle of a transition } \\
\text { period; if odd number of transition years: regime } \\
\text { change after }((1 / 2) n+1) / n) \text { years }\end{array}$ & $\begin{array}{l}\text { regime change from } \\
\text { DEM to AUT }\end{array}$ & $\begin{array}{l}\text { regime change from } \\
\text { AUT to DEM }\end{array}$ & Reich 2002 & & \\
\hline $\begin{array}{l}\text { Reich } 2002 \\
\text { (hybrid) }\end{array}$ & $\begin{array}{l}\text { DEM: democracy; HYB: semidemocracy; AUT: } \\
\text { autocracy; regime change with transitions--> } \\
\text { change takes place in the middle of a transition } \\
\text { period; if odd number of transition years: regime } \\
\text { change after }((1 / 2) n+1) / n) \text { years }\end{array}$ & $\begin{array}{l}\text { regime change from } \\
\text { DEM to AUT }\end{array}$ & $\begin{array}{l}\text { regime change from } \\
\text { AUT to DEM }\end{array}$ & Reich 2002 & Reich 2002 & Reich 2002 \\
\hline Svolik 2012 & author's coding of DEM and AUT & $\begin{array}{l}\text { regime change from } \\
\text { DEM to AUT }\end{array}$ & $\begin{array}{l}\text { regime change from } \\
\text { AUT to DEM }\end{array}$ & Svolik 2012 & Svolik 2012 & Svolik 2012 \\
\hline Ulfelder 2012 & author's coding of DEM and AUT & $\begin{array}{l}\text { regime change from } \\
\text { DEM to AUT }\end{array}$ & $\begin{array}{l}\text { regime change from } \\
\text { AUT to DEM }\end{array}$ & Ulfelder 2012 & & \\
\hline $\begin{array}{l}\text { Wahman et al. } \\
2013\end{array}$ & authors' coding of DEM and AUT & $\begin{array}{l}\text { regime change from } \\
\text { DEM to AUT }\end{array}$ & $\begin{array}{l}\text { regime change from } \\
\text { AUT to DEM }\end{array}$ & $\begin{array}{l}\text { Hadenius et al. } \\
2013\end{array}$ & & \\
\hline
\end{tabular}




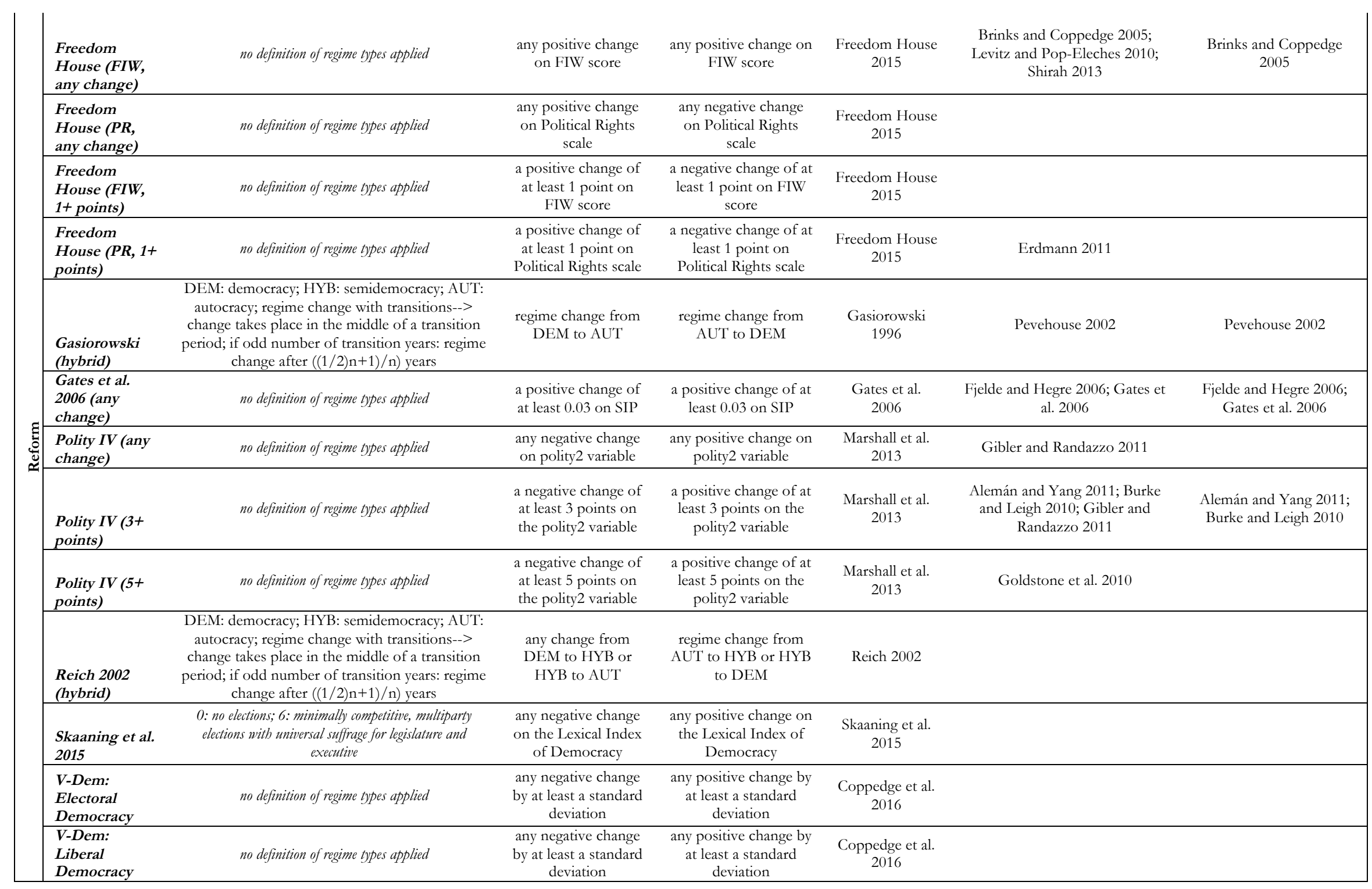




\begin{tabular}{|c|c|c|c|c|}
\hline $\begin{array}{l}\text { V-Dem: } \\
\text { Participatory } \\
\text { Democracy }\end{array}$ & no definition of regime types applied & $\begin{array}{c}\text { any negative change } \\
\text { by at least a standard } \\
\text { deviation }\end{array}$ & $\begin{array}{l}\text { any positive change by } \\
\text { at least a standard } \\
\text { deviation }\end{array}$ & $\begin{array}{c}\text { Coppedge et al. } \\
2016\end{array}$ \\
\hline $\begin{array}{l}\text { V-Dem: } \\
\text { Deliberative } \\
\text { Democracy }\end{array}$ & no definition of regime types applied & $\begin{array}{c}\text { any negative change } \\
\text { by at least a standard } \\
\text { deviation }\end{array}$ & $\begin{array}{l}\text { any positive change by } \\
\text { at least a standard } \\
\text { deviation }\end{array}$ & $\begin{array}{l}\text { Coppedge et al. } \\
2016\end{array}$ \\
\hline $\begin{array}{l}\text { V-Dem: } \\
\text { Egalitarian } \\
\text { Democracy }\end{array}$ & no definition of regime types applied & $\begin{array}{c}\text { any negative change } \\
\text { by at least a standard } \\
\text { deviation }\end{array}$ & $\begin{array}{l}\text { any positive change by } \\
\text { at least a standard } \\
\text { deviation }\end{array}$ & $\begin{array}{c}\text { Coppedge et al. } \\
2016\end{array}$ \\
\hline $\begin{array}{l}\text { V-Dem: } \\
\text { Electoral } \\
\text { Democracy } \\
\text { (ordinal) } \\
\end{array}$ & $\begin{array}{l}\text { no definition of regime types applied; } 5 \text { categories based } \\
\text { on continuous V-Dem indicators, following Lindberg } \\
\qquad 2015\end{array}$ & $\begin{array}{l}\text { any decrease on the } \\
\text { ordinal scale }\end{array}$ & $\begin{array}{l}\text { any increase on the } \\
\text { ordinal scale }\end{array}$ & $\begin{array}{l}\text { Coppedge et al. } \\
2016\end{array}$ \\
\hline $\begin{array}{l}\text { V-Dem: } \\
\text { Liberal } \\
\text { Democracy } \\
\text { (ordinal) } \\
\end{array}$ & $\begin{array}{c}\text { no definition of regime types applied; } 5 \text { categories based } \\
\text { on continuous V-Dem indicators, following Lindberg } \\
2015\end{array}$ & $\begin{array}{l}\text { any decrease on the } \\
\text { ordinal scale }\end{array}$ & $\begin{array}{l}\text { any increase on the } \\
\text { ordinal scale }\end{array}$ & $\begin{array}{c}\text { Coppedge et al. } \\
2016\end{array}$ \\
\hline $\begin{array}{l}\text { V-Dem: } \\
\text { Participatory } \\
\text { Democracy } \\
\text { (ordinal) }\end{array}$ & $\begin{array}{c}\text { no definition of regime types applied; } 5 \text { categories based } \\
\text { on continuous V-Dem indicators, following Lindberg } \\
2015\end{array}$ & $\begin{array}{l}\text { any decrease on the } \\
\text { ordinal scale }\end{array}$ & $\begin{array}{l}\text { any increase on the } \\
\text { ordinal scale }\end{array}$ & $\begin{array}{l}\text { Coppedge et al. } \\
2016\end{array}$ \\
\hline $\begin{array}{l}\text { V-Dem: } \\
\text { Deliberative } \\
\text { Democracy } \\
\text { (ordinal) } \\
\end{array}$ & $\begin{array}{c}\text { no definition of regime types applied; } 5 \text { categories based } \\
\text { on continuous V-Dem indicators, following Lindberg } \\
2015\end{array}$ & $\begin{array}{l}\text { any decrease on the } \\
\text { ordinal scale }\end{array}$ & $\begin{array}{l}\text { any increase on the } \\
\text { ordinal scale }\end{array}$ & $\begin{array}{l}\text { Coppedge et al. } \\
2016\end{array}$ \\
\hline $\begin{array}{l}\text { V-Dem: } \\
\text { Egalitarian } \\
\text { Democracy } \\
\text { (ordinal) } \\
\end{array}$ & $\begin{array}{c}\text { no definition of regime types applied; } 5 \text { categories based } \\
\text { on continuous V-Dem indicators, following Lindberg } \\
2015\end{array}$ & $\begin{array}{l}\text { any decrease on the } \\
\text { ordinal scale }\end{array}$ & $\begin{array}{l}\text { any increase on the } \\
\text { ordinal scale }\end{array}$ & $\begin{array}{c}\text { Coppedge et al. } \\
2016\end{array}$ \\
\hline $\begin{array}{l}\text { Wahman et al. } \\
2013 \text { (any } \\
\text { change) }\end{array}$ & no definition of regime types applied & $\begin{array}{l}\text { any negative change } \\
\text { on ifhpol variable } \\
\text { (imputed averaged } \\
\text { FH and Polity score) }\end{array}$ & $\begin{array}{l}\text { any positive change on } \\
\text { ifhpol variable } \\
\text { (imputed averaged FH } \\
\text { and Polity score) }\end{array}$ & $\begin{array}{l}\text { Hadenius et al. } \\
2013\end{array}$ \\
\hline $\begin{array}{l}\text { Wahman et al. } \\
2013(1.5+ \\
\text { points) }\end{array}$ & no definition of regime types applied & $\begin{array}{l}\text { a negative change of } \\
\text { at least } 1.5 \text { points on } \\
\text { ifhpol variable }\end{array}$ & $\begin{array}{l}\text { a positive change of at } \\
\text { least } 1.5 \text { points on } \\
\text { ifhpol variable }\end{array}$ & $\begin{array}{l}\text { Hadenius et al. } \\
2013\end{array}$ \\
\hline
\end{tabular}


Table A2: Indicators of Regime Change - Extended Set

\begin{tabular}{|c|c|c|c|c|c|c|c|c|c|}
\hline \multirow[b]{2}{*}{ Indicator name } & \multirow{3}{*}{$\begin{array}{c}\text { Period } \\
\text { available }\end{array}$} & \multicolumn{2}{|c|}{ full period } & \multicolumn{2}{|c|}{ post-WWII } & \multicolumn{2}{|c|}{ full period } & \multicolumn{2}{|c|}{ post-WWII } \\
\hline & & $\begin{array}{c}\# \\
\text { events }\end{array}$ & $\%$ obs & $\begin{array}{c}\# \\
\text { events }\end{array}$ & $\%$ obs & $\begin{array}{c}\# \\
\text { events }\end{array}$ & $\%$ obs & $\begin{array}{c}\# \\
\text { events }\end{array}$ & $\%$ obs \\
\hline & & \multicolumn{4}{|c|}{ TRANSITION } & \multicolumn{4}{|c|}{ BREAKDOWN } \\
\hline Bernhard et al. 2005 & $1913-2005$ & $\mathrm{NA}$ & $\mathrm{NA}$ & NA & $\mathrm{NA}$ & 63 & $1.73 \%$ & 54 & $1.69 \%$ \\
\hline Boix et al. 2013 & $1801-2007$ & 134 & $0.83 \%$ & 108 & $1.23 \%$ & 83 & $0.52 \%$ & 65 & $0.74 \%$ \\
\hline Cheibub et al. 2010 & $1947-2008$ & 102 & $1.14 \%$ & 102 & $1.14 \%$ & 66 & $0.74 \%$ & 66 & $0.74 \%$ \\
\hline Freedom House (FIW: free) & 1973-2015 & 91 & $1.19 \%$ & 91 & $1.19 \%$ & 72 & $0.94 \%$ & 72 & $0.94 \%$ \\
\hline Freedom House (FIW: partly free) & 1973-2015 & 150 & $1.97 \%$ & 150 & $1.97 \%$ & 129 & $1.69 \%$ & 129 & $1.69 \%$ \\
\hline Freedom House (PR: free) & 1973-2015 & 99 & $1.30 \%$ & 99 & $1.30 \%$ & 79 & $1.04 \%$ & 79 & $1.04 \%$ \\
\hline Freedom House (PR: partly free) & $1973-2015$ & 164 & $2.15 \%$ & 164 & $2.15 \%$ & 138 & $1.81 \%$ & 138 & $1.81 \%$ \\
\hline Freedom House (FIW: bybrid) & $1973-2015$ & 3 & $0.04 \%$ & 3 & $0.04 \%$ & 7 & $0.09 \%$ & 7 & $0.09 \%$ \\
\hline Freedom House (PR: bybrid) & 1973-2015 & 7 & $0.09 \%$ & 7 & $0.09 \%$ & 13 & $0.17 \%$ & 13 & $0.17 \%$ \\
\hline Gasiorowski 1996 & 1801-1992 & 84 & $1.27 \%$ & 73 & $1.98 \%$ & 72 & $1.09 \%$ & 67 & $1.81 \%$ \\
\hline Gasiorowski 1996 (bybrid) & 1801-1992 & 36 & $0.54 \%$ & 33 & $0.89 \%$ & 32 & $0.48 \%$ & 31 & $0.84 \%$ \\
\hline Gates 2006 & $1801-2000$ & 93 & $0.72 \%$ & 71 & $1.09 \%$ & 80 & $0.62 \%$ & 60 & $0.92 \%$ \\
\hline Geddes et al. 2014 & 1946-2009 & 103 & $1.32 \%$ & 103 & $1.32 \%$ & 75 & $0.96 \%$ & 75 & $0.96 \%$ \\
\hline Magaloni et al. 2013 & $1951-2012$ & 83 & $0.98 \%$ & 83 & $0.98 \%$ & 49 & $0.58 \%$ & 49 & $0.58 \%$ \\
\hline Polity IV $(A N=A U T)$ & $1801-2014$ & 131 & $0.80 \%$ & 106 & $1.17 \%$ & 80 & $0.49 \%$ & 64 & $0.71 \%$ \\
\hline Polity IV $(A N=D E M)$ & $1801-2014$ & 188 & $1.15 \%$ & 135 & $1.49 \%$ & 159 & $0.97 \%$ & 109 & $1.20 \%$ \\
\hline Polity IV (threshold: 7) & $1801-2014$ & 110 & $0.67 \%$ & 88 & $0.97 \%$ & 62 & $0.38 \%$ & 49 & $0.54 \%$ \\
\hline Polity IV (threshold: 1) & $1801-2014$ & 183 & $1.12 \%$ & 134 & $1.48 \%$ & 137 & $0.83 \%$ & 97 & $1.07 \%$ \\
\hline Polity IV (exrec \& parcomp) & $1801-2014$ & 112 & $0.72 \%$ & 83 & $0.96 \%$ & 86 & $0.55 \%$ & 67 & $0.77 \%$ \\
\hline Polity IV (bybrid: 6 \& -6) & $1801-2014$ & 18 & $0.11 \%$ & 17 & $0.19 \%$ & 26 & $0.16 \%$ & 20 & $0.22 \%$ \\
\hline Polity IV (bybrid: 8 \& 1) & $1801-2014$ & 24 & $0.15 \%$ & 18 & $0.20 \%$ & 24 & $0.15 \%$ & 19 & $0.21 \%$ \\
\hline Przeworski et al. 2000 & $1947-2002$ & 93 & $1.21 \%$ & 93 & $1.21 \%$ & 58 & $0.76 \%$ & 58 & $0.76 \%$ \\
\hline Reich 2002 & 1801-1998 & 131 & $1.22 \%$ & 106 & $1.79 \%$ & 92 & $0.86 \%$ & 73 & $1.23 \%$ \\
\hline Reich 2002 (bybrid) & 1801-1998 & 52 & $0.48 \%$ & 44 & $0.74 \%$ & 40 & $0.37 \%$ & 33 & $0.56 \%$ \\
\hline Svolik 2012 & $1946-2007$ & 89 & $1.07 \%$ & 89 & $1.07 \%$ & 57 & $0.69 \%$ & 57 & $0.69 \%$ \\
\hline Ulfelder 2012 & $1956-2010$ & 113 & $1.51 \%$ & 113 & $1.51 \%$ & 109 & $1.45 \%$ & 109 & $1.45 \%$ \\
\hline \multirow[t]{2}{*}{ Wabman et al. 2013} & $1973-2010$ & 98 & $1.51 \%$ & 98 & $1.51 \%$ & 62 & $0.95 \%$ & 62 & $0.95 \%$ \\
\hline & & \multicolumn{4}{|c|}{ LIBERALIZATION } & \multicolumn{4}{|c|}{ BACKSLIDING } \\
\hline Freedom House (FIW, any change) & 1973-2015 & 919 & $12.04 \%$ & 919 & $\begin{array}{c}12.04 \\
\%\end{array}$ & 738 & $9.67 \%$ & 738 & $9.67 \%$ \\
\hline Freedom House (PR, any change) & 1973-2015 & 581 & $7.61 \%$ & 581 & $7.61 \%$ & 469 & $6.15 \%$ & 469 & $6.15 \%$ \\
\hline Freedom House (FIW, 1+ points) & 1973-2015 & 247 & $3.24 \%$ & 247 & $3.24 \%$ & 175 & $2.29 \%$ & 175 & $2.29 \%$ \\
\hline Freedom House (PR, 1+ points) & $1973-2015$ & 561 & $7.35 \%$ & 561 & $7.35 \%$ & 451 & $5.91 \%$ & 451 & $5.91 \%$ \\
\hline Gasiorowski (bybrid) & 1801-1992 & 56 & $0.84 \%$ & 47 & $1.27 \%$ & 42 & $0.65 \%$ & 39 & $1.06 \%$ \\
\hline Gates et al. 2006 (any change) & $1801-2000$ & 418 & $3.25 \%$ & 245 & $3.76 \%$ & 302 & $2.35 \%$ & 192 & $2.95 \%$ \\
\hline Polity IV (any change) & $1801-2014$ & 811 & $4.94 \%$ & 556 & $6.13 \%$ & 511 & $3.11 \%$ & 341 & $3.76 \%$ \\
\hline Polity IV (3+ points) & $1801-2014$ & 368 & $2.24 \%$ & 264 & $2.91 \%$ & 266 & $1.62 \%$ & 176 & $1.94 \%$ \\
\hline Polity IV (5+ points) & $1801-2014$ & 218 & $1.33 \%$ & 162 & $1.79 \%$ & 156 & $0.95 \%$ & 112 & $1.24 \%$ \\
\hline Reich 2002 (bybrid) & $1801-1998$ & 104 & $0.97 \%$ & 77 & $1.30 \%$ & 60 & $0.56 \%$ & 47 & $0.79 \%$ \\
\hline Skaaning et al. 2015 & $1801-2015$ & 657 & $3.80 \%$ & 379 & $3.66 \%$ & 483 & $2.79 \%$ & 261 & $2.52 \%$ \\
\hline$V$-Dem: Electoral Democracy & $1901-2015$ & 803 & $5.05 \%$ & 669 & $6.50 \%$ & 400 & $2.52 \%$ & 302 & $2.93 \%$ \\
\hline$V$-Dem: Liberal Democracy & $1901-2015$ & 702 & $4.42 \%$ & 596 & $5.79 \%$ & 371 & $2.33 \%$ & 294 & $2.85 \%$ \\
\hline V-Dem: Participatory Democracy & $1901-2015$ & 849 & $5.34 \%$ & 748 & $7.27 \%$ & 409 & $2.57 \%$ & 328 & $3.19 \%$ \\
\hline$V$-Dem: Deliberative Democracy & $1901-2015$ & 737 & $4.67 \%$ & 637 & $6.23 \%$ & 398 & $2.52 \%$ & 326 & $3.19 \%$ \\
\hline V-Dem: Egalitarian Democracy & $1901-2015$ & 729 & $4.59 \%$ & 634 & $6.16 \%$ & 323 & $2.03 \%$ & 265 & $2.57 \%$ \\
\hline V-Dem: Electoral Democracy (ordinal) & $1901-2015$ & 586 & $3.68 \%$ & 503 & $4.88 \%$ & 350 & $2.20 \%$ & 273 & $2.65 \%$ \\
\hline V-Dem: Liberal Democracy (ordinal) & $1901-2015$ & 381 & $2.40 \%$ & 323 & $3.14 \%$ & 198 & $1.25 \%$ & 153 & $1.49 \%$ \\
\hline$V$-Dem: Participatory Democracy & $1901-2015$ & 330 & $2.08 \%$ & 288 & $2.80 \%$ & 155 & $0.98 \%$ & 121 & $1.18 \%$ \\
\hline
\end{tabular}




\begin{tabular}{|c|c|c|c|c|c|c|c|c|c|}
\hline \multicolumn{10}{|l|}{ (ordinal) } \\
\hline V-Dem: Deliberative Democracy (ordinal) & $1901-2015$ & 416 & $2.63 \%$ & 359 & $3.51 \%$ & 234 & $1.48 \%$ & 191 & $1.87 \%$ \\
\hline V-Dem: Egalitarian Democracy (ordinal) & $1901-2015$ & 381 & $2.40 \%$ & 331 & $3.21 \%$ & 187 & $1.18 \%$ & 150 & $1.46 \%$ \\
\hline Wabman et al. 2013 (any change) & 1973-2010 & 1033 & $15.87 \%$ & 1033 & $\begin{array}{c}15.87 \\
\%\end{array}$ & 730 & $\begin{array}{c}11.21 \\
\%\end{array}$ & 730 & $\begin{array}{c}11.21 \\
\%\end{array}$ \\
\hline Wabman et al. 2013 (1.5+ points) & $1973-2010$ & 173 & $2.66 \%$ & 173 & $2.66 \%$ & 89 & $1.37 \%$ & 89 & $1.37 \%$ \\
\hline
\end{tabular}


Table A3: Pairwise correlation coefficients between indicators of regime type

a) core indicators: V-Dem continuous

\begin{tabular}{|c|c|c|c|c|c|c|c|c|c|c|c|c|c|c|c|}
\hline & 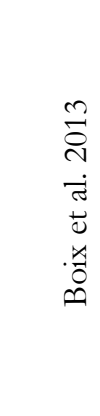 & 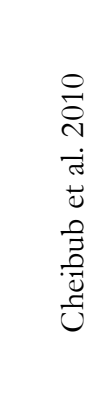 & 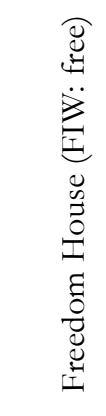 & 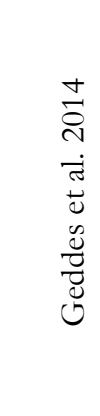 & 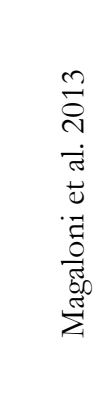 & $\begin{array}{l}\nexists \\
\stackrel{B}{E} \\
0 \\
0 \\
0\end{array}$ & 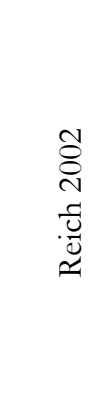 & 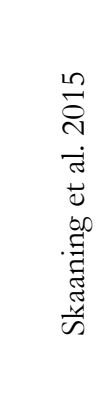 & 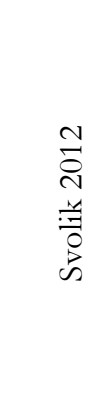 & 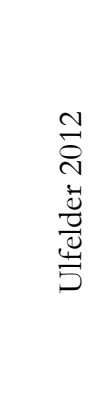 & 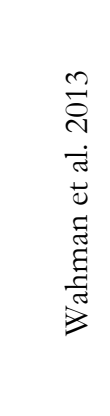 & 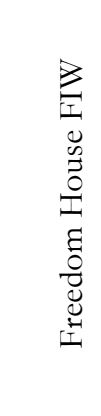 & 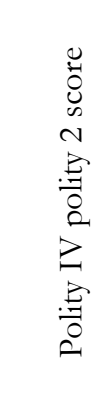 & 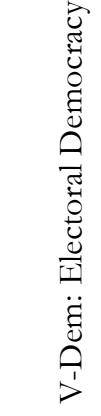 & 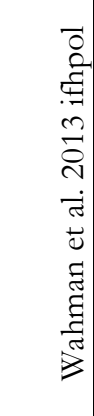 \\
\hline Boix et al. 2013 & 1.00 & & & & & & & & & & & & & & \\
\hline Cheibub et al. 2010 & 0.92 & 1.00 & & & & & & & & & & & & & \\
\hline Freedom House (FIW: free) & 0.81 & 0.74 & 1.00 & & & & & & & & & & & & \\
\hline Geddes et al. 2014 & 0.89 & 0.92 & 0.76 & 1.00 & & & & & & & & & & & \\
\hline Magaloni et al. 2013 & 0.91 & 0.92 & 0.76 & 0.94 & 1.00 & & & & & & & & & & \\
\hline Polity IV & 0.84 & 0.79 & 0.78 & 0.85 & 0.86 & 1.00 & & & & & & & & & \\
\hline Reich 2002 & 0.75 & 0.82 & 0.72 & 0.84 & 0.83 & 0.70 & 1.00 & & & & & & & & \\
\hline Skaaning et al. 2015 & 0.89 & 0.88 & 0.76 & 0.89 & 0.90 & 0.83 & 0.78 & 1.00 & & & & & & & \\
\hline Svolik 2012 & 0.91 & 0.92 & 0.77 & 0.93 & 0.93 & 0.84 & 0.85 & 0.91 & 1.00 & & & & & & \\
\hline Ulfelder 2012 & 0.87 & 0.84 & 0.76 & 0.87 & 0.86 & 0.84 & 0.86 & 0.89 & 0.90 & 1.00 & & & & & \\
\hline Wahman et al. 2013 & 0.87 & 0.81 & 0.89 & 0.85 & 0.86 & 0.91 & 0.78 & 0.87 & 0.85 & 0.84 & 1.00 & & & & \\
\hline Freedom House FIW & 0.86 & 0.83 & 0.86 & 0.83 & 0.83 & 0.84 & 0.83 & 0.85 & 0.85 & 0.84 & 0.88 & 1.00 & & & \\
\hline Polity IV polity 2 score & 0.82 & 0.84 & 0.75 & 0.87 & 0.86 & 0.85 & 0.75 & 0.83 & 0.88 & 0.87 & 0.85 & 0.89 & 1.00 & & \\
\hline V-Dem: Electoral Democracy & 0.84 & 0.83 & 0.81 & 0.85 & 0.84 & 0.84 & 0.81 & 0.83 & 0.85 & 0.84 & 0.85 & 0.91 & 0.87 & 1.00 & \\
\hline Wahman et al. 2013 ifhpol & 0.88 & 0.85 & 0.84 & 0.87 & 0.87 & 0.88 & 0.88 & 0.90 & 0.89 & 0.88 & 0.89 & 0.97 & 0.97 & 0.93 & 1.00 \\
\hline average & 0.86 & 0.85 & 0.79 & 0.87 & 0.87 & 0.83 & 0.80 & 0.86 & 0.88 & 0.85 & 0.86 & 0.86 & 0.85 & 0.85 & 0.89 \\
\hline
\end{tabular}


b) core indicators: V-Dem ordinal

\begin{tabular}{|c|c|c|c|c|c|c|c|c|c|c|c|c|c|c|c|}
\hline & $\begin{array}{l}m \\
\stackrel{\sim}{0} \\
\dot{\sim} \\
\tilde{\sigma} \\
\Delta \\
.4 \\
0 \\
\infty\end{array}$ & 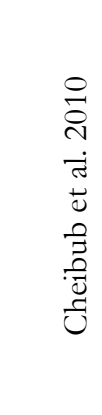 & 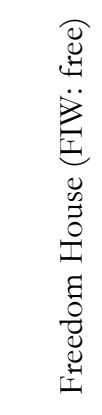 & 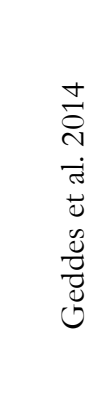 & 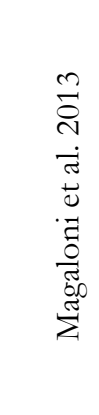 & $\begin{array}{l}Z \\
: \\
0 \\
0\end{array}$ & 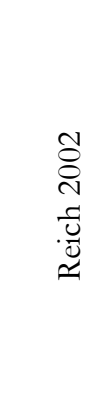 & 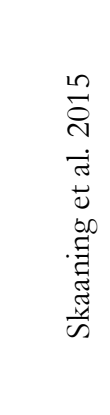 & 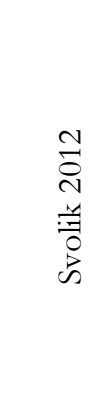 & 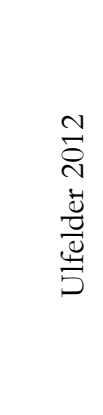 & 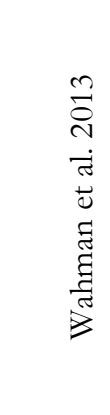 & 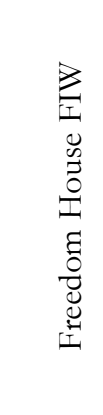 & 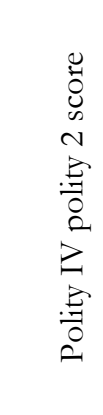 & 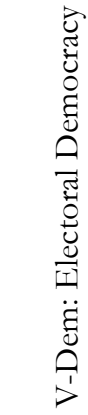 & 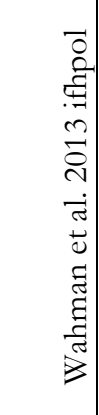 \\
\hline Boix et al. 2013 & 1.00 & & & & & & & & & & & & & & \\
\hline Cheibub et al. 2010 & 0.92 & 1.00 & & & & & & & & & & & & & \\
\hline Freedom House (FIW: free) & 0.81 & 0.74 & 1.00 & & & & & & & & & & & & \\
\hline Geddes et al. 2014 & 0.89 & 0.92 & 0.76 & 1.00 & & & & & & & & & & & \\
\hline Magaloni et al. 2013 & 0.91 & 0.92 & 0.76 & 0.94 & 1.00 & & & & & & & & & & \\
\hline Polity IV & 0.84 & 0.79 & 0.78 & 0.85 & 0.86 & 1.00 & & & & & & & & & \\
\hline Reich 2002 & 0.75 & 0.82 & 0.72 & 0.84 & 0.83 & 0.70 & 1.00 & & & & & & & & \\
\hline Skaaning et al. 2015 & 0.89 & 0.88 & 0.76 & 0.89 & 0.90 & 0.83 & 0.78 & 1.00 & & & & & & & \\
\hline Svolik 2012 & 0.91 & 0.92 & 0.77 & 0.93 & 0.93 & 0.84 & 0.85 & 0.91 & 1.00 & & & & & & \\
\hline Ulfelder 2012 & 0.87 & 0.84 & 0.76 & 0.87 & 0.86 & 0.84 & 0.86 & 0.89 & 0.90 & 1.00 & & & & & \\
\hline Wahman et al. 2013 & 0.87 & 0.81 & 0.89 & 0.85 & 0.86 & 0.91 & 0.78 & 0.87 & 0.85 & 0.84 & 1.00 & & & & \\
\hline Freedom House FIW & 0.86 & 0.83 & 0.86 & 0.83 & 0.83 & 0.84 & 0.83 & 0.85 & 0.85 & 0.84 & 0.88 & 1.00 & & & \\
\hline Polity IV polity 2 score & 0.82 & 0.84 & 0.75 & 0.87 & 0.86 & 0.85 & 0.75 & 0.83 & 0.88 & 0.87 & 0.85 & 0.89 & 1.00 & & \\
\hline V-Dem: Electoral Democracy (ordinal) & 0.83 & 0.82 & 0.80 & 0.84 & 0.83 & 0.82 & 0.80 & 0.83 & 0.84 & 0.83 & 0.84 & 0.90 & 0.85 & 1.00 & \\
\hline Wahman et al. 2013 ifhpol & 0.88 & 0.85 & 0.84 & 0.87 & 0.87 & 0.88 & 0.88 & 0.90 & 0.89 & 0.88 & 0.89 & 0.97 & 0.97 & 0.92 & 1.00 \\
\hline average & 0.86 & 0.85 & 0.79 & 0.87 & 0.87 & 0.83 & 0.80 & 0.86 & 0.88 & 0.85 & 0.86 & 0.86 & 0.85 & 0.84 & 0.89 \\
\hline
\end{tabular}


c) extended set of indicators-V-Dem continuous

\begin{tabular}{|c|c|c|c|c|c|c|c|c|c|c|c|c|c|c|c|c|c|c|c|c|c|c|c|c|c|c|c|c|c|c|}
\hline & 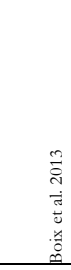 & 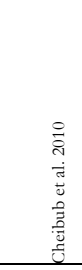 & 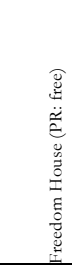 & 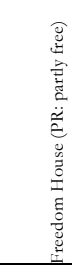 & 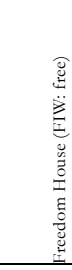 & 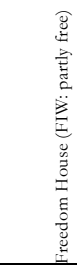 & 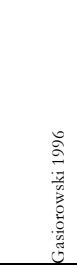 & 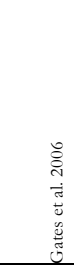 & 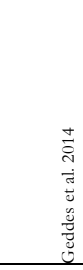 & 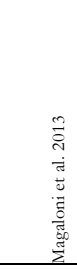 & 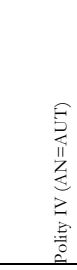 & 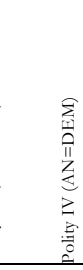 & 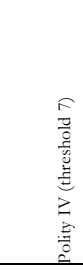 & 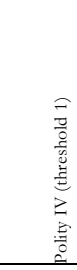 & 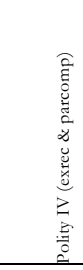 & 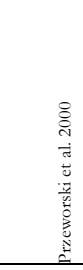 & 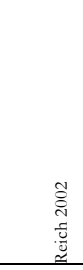 & 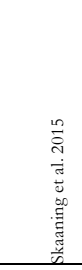 & 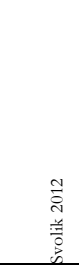 & 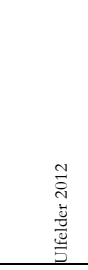 & 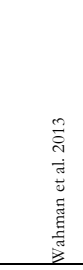 & 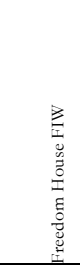 & 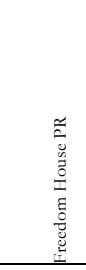 & 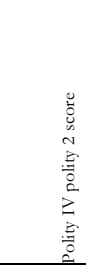 & 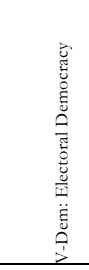 & 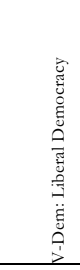 & 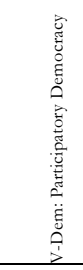 & 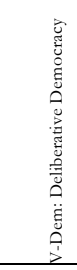 & 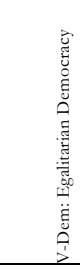 & \\
\hline Boix et al. 2013 & 1.00 & & & & & & & & & & & & & & & & & & & & & & & & & & & & & \\
\hline Cheibub et al. 2010 & 0.92 & 1.00 & & & & & & & & & & & & & & & & & & & & & & & & & & & & \\
\hline Freedom House (PR: free) & 0.82 & 0.75 & 1.00 & & & & & & & & & & & & & & & & & & & & & & & & & & & \\
\hline Freedom House (PR: partly free) & 0.66 & 0.65 & 0.56 & 1.00 & & & & & & & & & & & & & & & & & & & & & & & & & & \\
\hline Freedom House (FIV: free) & 0.81 & 0.74 & 0.97 & 0.56 & 1.00 & & & & & & & & & & & & & & & & & & & & & & & & & \\
\hline Freedom House (FIW: partly free) & 0.61 & 0.60 & 0.53 & 0.91 & 0.52 & 1.00 & & & & & & & & & & & & & & & & & & & & & & & & \\
\hline Gasiorowski 1996 & 0.67 & 0.75 & 0.73 & 0.56 & 0.71 & 0.50 & 1.00 & & & & & & & & & & & & & & & & & & & & & & & \\
\hline Gates et al. 2006 & 0.85 & 0.83 & 0.81 & 0.68 & 0.78 & 0.62 & 0.63 & 1.00 & & & & & & & & & & & & & & & & & & & & & & \\
\hline Geddes et al. 2014 & 0.89 & 0.92 & 0.76 & 0.65 & 0.76 & 0.60 & 0.77 & 0.86 & 1.00 & & & & & & & & & & & & & & & & & & & & & \\
\hline Magaloni et al. 2013 & 0.91 & 0.92 & 0.77 & 0.64 & 0.76 & 0.59 & 0.77 & 0.86 & 0.94 & 1.00 & & & & & & & & & & & & & & & & & & & & \\
\hline Polity IV (AN=AUT) & 0.84 & 0.79 & 0.78 & 0.65 & 0.78 & 0.60 & 0.58 & 0.85 & 0.85 & 0.86 & 1.00 & & & & & & & & & & & & & & & & & & & \\
\hline Polity IV (AN=DEM) & 0.51 & 0.66 & 0.51 & 0.64 & 0.51 & 0.61 & 0.39 & 0.55 & 0.65 & 0.63 & 0.48 & 1.00 & & & & & & & & & & & & & & & & & & \\
\hline Polity IV (threshold 7) & 0.82 & 0.76 & 0.78 & 0.58 & 0.78 & 0.54 & 0.54 & 0.82 & 0.81 & 0.82 & 0.93 & 0.45 & 1.00 & & & & & & & & & & & & & & & & & \\
\hline Polity IV (threshold 1) & 0.76 & 0.82 & 0.68 & 0.71 & 0.68 & 0.66 & 0.60 & 0.85 & 0.84 & 0.83 & 0.76 & 0.63 & 0.71 & 1.00 & & & & & & & & & & & & & & & & \\
\hline Polity IV (exrec \& parcomp) & 0.79 & 0.82 & 0.70 & 0.71 & 0.70 & 0.66 & 0.64 & 0.83 & 0.85 & 0.84 & 0.84 & 0.58 & 0.78 & 0.86 & 1.00 & & & & & & & & & & & & & & & \\
\hline Przeworski et al. 2000 & 0.92 & 0.96 & 0.80 & 0.65 & 0.78 & 0.59 & 0.75 & 0.84 & 0.92 & 0.92 & 0.80 & 0.67 & 0.76 & 0.83 & 0.82 & 1.00 & & & & & & & & & & & & & & \\
\hline Reich 2002 & 0.75 & 0.82 & 0.73 & 0.70 & 0.72 & 0.64 & 0.99 & 0.73 & 0.84 & 0.83 & 0.70 & 0.52 & 0.66 & 0.70 & 0.71 & 0.83 & 1.00 & & & & & & & & & & & & & \\
\hline Skaaning et al. 2015 & 0.89 & 0.88 & 0.76 & 0.69 & 0.76 & 0.64 & 0.68 & 0.85 & 0.89 & 0.90 & 0.83 & 0.54 & 0.78 & 0.79 & 0.79 & 0.89 & 0.78 & 1.00 & & & & & & & & & & & & \\
\hline Svolik 2012 & 0.91 & 0.92 & 0.78 & 0.68 & 0.77 & 0.62 & 0.77 & 0.87 & 0.93 & 0.93 & 0.84 & 0.68 & 0.79 & 0.86 & 0.85 & 0.94 & 0.85 & 0.91 & 1.00 & & & & & & & & & & & \\
\hline Ulfelder 2012 & 0.87 & 0.84 & 0.77 & 0.66 & 0.76 & 0.61 & 0.83 & 0.89 & 0.87 & 0.86 & 0.84 & 0.68 & 0.78 & 0.85 & 0.85 & 0.87 & 0.86 & 0.89 & 0.90 & 1.00 & & & & & & & & & & \\
\hline Wahman et al. 2013 & 0.87 & 0.81 & 0.89 & 0.62 & 0.89 & 0.58 & 0.77 & 0.86 & 0.85 & 0.86 & 0.91 & 0.60 & 0.88 & 0.80 & 0.81 & 0.82 & 0.78 & 0.87 & 0.85 & 0.84 & 1.00 & & & & & & & & & \\
\hline Freedom House FIW & 0.87 & 0.84 & 0.87 & 0.83 & 0.86 & 0.78 & 0.78 & 0.87 & 0.84 & 0.84 & 0.85 & 0.69 & 0.81 & 0.83 & 0.84 & 0.85 & 0.84 & 0.87 & 0.86 & 0.85 & 0.88 & 1.00 & & & & & & & & \\
\hline Freedom House PR & 0.86 & 0.83 & 0.86 & 0.81 & 0.86 & 0.79 & 0.77 & 0.86 & 0.83 & 0.83 & 0.84 & 0.70 & 0.81 & 0.82 & 0.83 & 0.83 & 0.83 & 0.85 & 0.85 & 0.84 & 0.88 & 0.98 & 1.00 & & & & & & & \\
\hline Polity IV polity 2 score & 0.82 & 0.84 & 0.76 & 0.73 & 0.75 & 0.69 & 0.64 & 0.87 & 0.87 & 0.86 & 0.85 & 0.78 & 0.81 & 0.91 & 0.87 & 0.85 & 0.75 & 0.83 & 0.88 & 0.87 & 0.85 & 0.89 & 0.89 & 1.00 & & & & & & \\
\hline V-Dem: Electoral D. & 0.84 & 0.83 & 0.81 & 0.70 & 0.81 & 0.66 & 0.74 & 0.82 & 0.85 & 0.84 & 0.84 & 0.63 & 0.81 & 0.77 & 0.79 & 0.84 & 0.81 & 0.83 & 0.85 & 0.84 & 0.85 & 0.90 & 0.91 & 0.87 & 1.00 & & & & & \\
\hline V-Dem: Liberal D. & 0.83 & 0.81 & 0.83 & 0.65 & 0.84 & 0.62 & 0.73 & 0.80 & 0.83 & 0.83 & 0.83 & 0.58 & 0.83 & 0.74 & 0.76 & 0.81 & 0.79 & 0.82 & 0.83 & 0.81 & 0.85 & 0.89 & 0.91 & 0.84 & 0.97 & 1.00 & & & & \\
\hline V-Dem: Participatory D. & 0.82 & 0.80 & 0.80 & 0.65 & 0.81 & 0.62 & 0.69 & 0.78 & 0.82 & 0.82 & 0.82 & 0.58 & 0.81 & 0.73 & 0.76 & 0.81 & 0.77 & 0.81 & 0.82 & 0.79 & 0.83 & 0.88 & 0.89 & 0.83 & 0.97 & 0.97 & 1.00 & & & \\
\hline V-Dem: Deliberative D. & 0.83 & 0.81 & 0.82 & 0.66 & 0.82 & 0.62 & 0.72 & 0.79 & 0.82 & 0.82 & 0.82 & 0.58 & 0.81 & 0.74 & 0.77 & 0.81 & 0.79 & 0.81 & 0.83 & 0.81 & 0.84 & 0.88 & 0.89 & 0.84 & 0.97 & 0.98 & 0.98 & 1.00 & & \\
\hline V-Dem: Egalitarian D. & 0.78 & 0.75 & 0.81 & 0.59 & 0.82 & 0.56 & 0.63 & 0.73 & 0.77 & 0.77 & 0.79 & 0.49 & 0.79 & 0.67 & 0.70 & 0.76 & 0.73 & 0.75 & 0.77 & 0.74 & 0.80 & 0.84 & 0.86 & 0.76 & 0.95 & 0.97 & 0.96 & 0.96 & 1.00 & \\
\hline Wahman et al. 2013 ifhpol & 0.88 & 0.85 & 0.84 & 0.79 & 0.84 & 0.76 & 0.84 & 0.92 & 0.87 & 0.87 & 0.88 & 0.80 & 0.84 & 0.91 & 0.90 & 0.86 & 0.88 & 0.90 & 0.89 & 0.88 & 0.89 & 0.97 & 0.97 & 0.97 & 0.93 & 0.90 & 0.89 & 0.90 & 0.85 & 1.00 \\
\hline average & 0.81 & 0.81 & 0.77 & 0.67 & 0.76 & 0.63 & 0.70 & 0.80 & 0.83 & 0.83 & 0.79 & 0.60 & 0.76 & 0.77 & 0.78 & 0.82 & 0.77 & 0.81 & 0.84 & 0.82 & 0.82 & 0.86 & 0.85 & 0.83 & 0.84 & 0.82 & 0.81 & 0.82 & 0.77 & 0.88 \\
\hline
\end{tabular}


d) extended set of indicators-V-Dem ordinal

\begin{tabular}{|c|c|c|c|c|c|c|c|c|c|c|c|c|c|c|c|c|c|c|c|c|c|c|c|c|c|c|c|c|c|c|}
\hline & 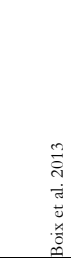 & 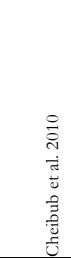 & 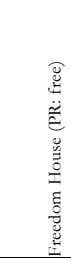 & 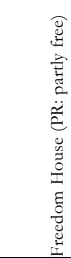 & 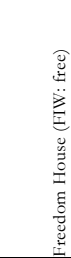 & 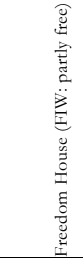 & 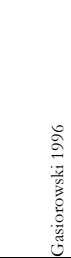 & 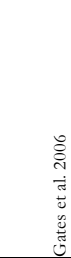 & 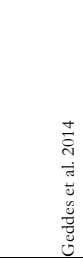 & 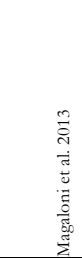 & 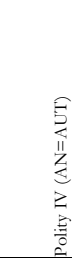 & 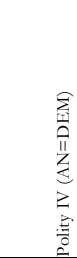 & 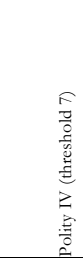 & 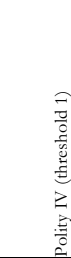 & 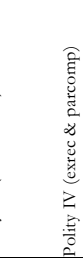 & 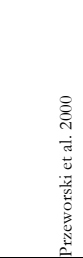 & 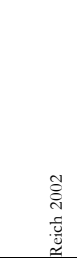 & 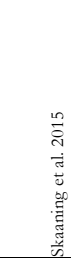 & 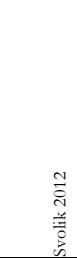 & 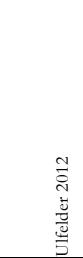 & 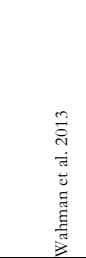 & 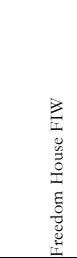 & 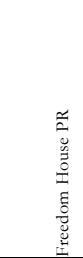 & 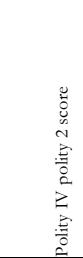 & 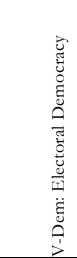 & 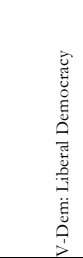 & 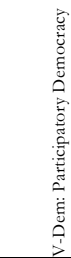 & 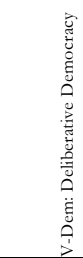 & 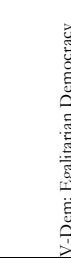 & 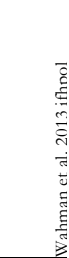 \\
\hline Boix et al. 2013 & 1.00 & & & & & & & & & & & & & & & & & & & & & & & & & & & & & \\
\hline Cheibub et al. 2010 & 0.92 & 1.00 & & & & & & & & & & & & & & & & & & & & & & & & & & & & \\
\hline Freedom House (PR: free) & 0.82 & 0.75 & 1.00 & & & & & & & & & & & & & & & & & & & & & & & & & & & \\
\hline Freedom House (PR: partly free) & 0.66 & 0.65 & 0.56 & 1.00 & & & & & & & & & & & & & & & & & & & & & & & & & & \\
\hline Freedom House (FIV: free) & 0.81 & 0.74 & 0.97 & 0.56 & 1.00 & & & & & & & & & & & & & & & & & & & & & & & & & \\
\hline Freedom House (FIW: partly free) & 0.61 & 0.60 & 0.53 & 0.91 & 0.52 & 1.00 & & & & & & & & & & & & & & & & & & & & & & & & \\
\hline Gasiorowski 1996 & 0.67 & 0.75 & 0.73 & 0.56 & 0.71 & 0.50 & 1.00 & & & & & & & & & & & & & & & & & & & & & & & \\
\hline Gates et al. 2006 & 0.85 & 0.83 & 0.81 & 0.68 & 0.78 & 0.62 & 0.63 & 1.00 & & & & & & & & & & & & & & & & & & & & & & \\
\hline Geddes et al. 2014 & 0.89 & 0.92 & 0.76 & 0.65 & 0.76 & 0.60 & 0.77 & 0.86 & 1.00 & & & & & & & & & & & & & & & & & & & & & \\
\hline Magaloni et al. 2013 & 0.91 & 0.92 & 0.77 & 0.64 & 0.76 & 0.59 & 0.77 & 0.86 & 0.94 & 1.00 & & & & & & & & & & & & & & & & & & & & \\
\hline Polity IV (AN=AUT) & 0.84 & 0.79 & 0.78 & 0.65 & 0.78 & 0.60 & 0.58 & 0.85 & 0.85 & 0.86 & 1.00 & & & & & & & & & & & & & & & & & & & \\
\hline Polity IV (AN=DEM) & 0.51 & 0.66 & 0.51 & 0.64 & 0.51 & 0.61 & 0.39 & 0.55 & 0.65 & 0.63 & 0.48 & 1.00 & & & & & & & & & & & & & & & & & & \\
\hline Polity IV (threshold 7) & 0.82 & 0.76 & 0.78 & 0.58 & 0.78 & 0.54 & 0.54 & 0.82 & 0.81 & 0.82 & 0.93 & 0.45 & 1.00 & & & & & & & & & & & & & & & & & \\
\hline Polity IV (threshold 1) & 0.76 & 0.82 & 0.68 & 0.71 & 0.68 & 0.66 & 0.60 & 0.85 & 0.84 & 0.83 & 0.76 & 0.63 & 0.71 & 1.00 & & & & & & & & & & & & & & & & \\
\hline Polity IV (exrec \& parcomp) & 0.79 & 0.82 & 0.70 & 0.71 & 0.70 & 0.66 & 0.64 & 0.83 & 0.85 & 0.84 & 0.84 & 0.58 & 0.78 & 0.86 & 1.00 & & & & & & & & & & & & & & & \\
\hline Przeworski et al. 2000 & 0.92 & 0.96 & 0.80 & 0.65 & 0.78 & 0.59 & 0.75 & 0.84 & 0.92 & 0.92 & 0.80 & 0.67 & 0.76 & 0.83 & 0.82 & 1.00 & & & & & & & & & & & & & & \\
\hline Reich 2002 & 0.75 & 0.82 & 0.73 & 0.70 & 0.72 & 0.64 & 0.99 & 0.73 & 0.84 & 0.83 & 0.70 & 0.52 & 0.66 & 0.70 & 0.71 & 0.83 & 1.00 & & & & & & & & & & & & & \\
\hline Skaaning et al. 2015 & 0.89 & 0.88 & 0.76 & 0.69 & 0.76 & 0.64 & 0.68 & 0.85 & 0.89 & 0.90 & 0.83 & 0.54 & 0.78 & 0.79 & 0.79 & 0.89 & 0.78 & 1.00 & & & & & & & & & & & & \\
\hline Svolik 2012 & 0.91 & 0.92 & 0.78 & 0.68 & 0.77 & 0.62 & 0.77 & 0.87 & 0.93 & 0.93 & 0.84 & 0.68 & 0.79 & 0.86 & 0.85 & 0.94 & 0.85 & 0.91 & 1.00 & & & & & & & & & & & \\
\hline Ulfelder 2012 & 0.87 & 0.84 & 0.77 & 0.66 & 0.76 & 0.61 & 0.83 & 0.89 & 0.87 & 0.86 & 0.84 & 0.68 & 0.78 & 0.85 & 0.85 & 0.87 & 0.86 & 0.89 & 0.90 & 1.00 & & & & & & & & & & \\
\hline Wahman et al. 2013 & 0.87 & 0.81 & 0.89 & 0.62 & 0.89 & 0.58 & 0.77 & 0.86 & 0.85 & 0.86 & 0.91 & 0.60 & 0.88 & 0.80 & 0.81 & 0.82 & 0.78 & 0.87 & 0.85 & 0.84 & 1.00 & & & & & & & & & \\
\hline Freedom House FIW & 0.87 & 0.84 & 0.87 & 0.83 & 0.86 & 0.78 & 0.78 & 0.87 & 0.84 & 0.84 & 0.85 & 0.69 & 0.81 & 0.83 & 0.84 & 0.85 & 0.84 & 0.87 & 0.86 & 0.85 & 0.88 & 1.00 & & & & & & & & \\
\hline Freedom House PR & 0.86 & 0.83 & 0.86 & 0.81 & 0.86 & 0.79 & 0.77 & 0.86 & 0.83 & 0.83 & 0.84 & 0.70 & 0.81 & 0.82 & 0.83 & 0.83 & 0.83 & 0.85 & 0.85 & 0.84 & 0.88 & 0.98 & 1.00 & & & & & & & \\
\hline Polity IV polity 2 score & 0.82 & 0.84 & 0.76 & 0.73 & 0.75 & 0.69 & 0.64 & 0.87 & 0.87 & 0.86 & 0.85 & 0.78 & 0.81 & 0.91 & 0.87 & 0.85 & 0.75 & 0.83 & 0.88 & 0.87 & 0.85 & 0.89 & 0.89 & 1.00 & & & & & & \\
\hline V-Dem: Electoral D. (ordinal) & 0.83 & 0.82 & 0.80 & 0.69 & 0.80 & 0.65 & 0.72 & 0.81 & 0.84 & 0.83 & 0.82 & 0.62 & 0.80 & 0.76 & 0.78 & 0.83 & 0.80 & 0.83 & 0.84 & 0.83 & 0.84 & 0.89 & 0.90 & 0.85 & 1.00 & & & & & \\
\hline V-Dem: Liberal D. (ordinal) & 0.82 & 0.80 & 0.83 & 0.63 & 0.83 & 0.59 & 0.73 & 0.78 & 0.82 & 0.82 & 0.82 & 0.55 & 0.82 & 0.72 & 0.75 & 0.81 & 0.78 & 0.81 & 0.82 & 0.80 & 0.84 & 0.87 & 0.89 & 0.82 & 0.94 & 1.00 & & & & \\
\hline V-Dem: Participatory D. (ordinal) & 0.81 & 0.78 & 0.80 & 0.62 & 0.80 & 0.58 & 0.64 & 0.75 & 0.80 & 0.80 & 0.79 & 0.53 & 0.79 & 0.69 & 0.73 & 0.78 & 0.74 & 0.78 & 0.79 & 0.77 & 0.82 & 0.85 & 0.86 & 0.79 & 0.93 & 0.94 & 1.00 & & & \\
\hline V-Dem: Deliberative D. (ordinal) & 0.80 & 0.78 & 0.81 & 0.62 & 0.82 & 0.58 & 0.65 & 0.75 & 0.80 & 0.81 & 0.81 & 0.53 & 0.80 & 0.70 & 0.74 & 0.79 & 0.74 & 0.78 & 0.80 & 0.78 & 0.83 & 0.86 & 0.87 & 0.80 & 0.93 & 0.95 & 0.95 & 1.00 & & \\
\hline V-Dem: Egalitarian D. (ordinal) & 0.75 & 0.73 & 0.78 & 0.58 & 0.79 & 0.54 & 0.59 & 0.71 & 0.75 & 0.75 & 0.76 & 0.48 & 0.77 & 0.65 & 0.68 & 0.73 & 0.70 & 0.73 & 0.75 & 0.72 & 0.78 & 0.82 & 0.84 & 0.75 & 0.91 & 0.94 & 0.93 & 0.94 & 1.00 & \\
\hline Wahman et al. 2013 iffpol & 0.88 & 0.85 & 0.84 & 0.79 & 0.84 & 0.76 & 0.84 & 0.92 & 0.87 & 0.87 & 0.88 & 0.80 & 0.84 & 0.91 & 0.90 & 0.86 & 0.88 & 0.90 & 0.89 & 0.88 & 0.89 & 0.97 & 0.97 & 0.97 & 0.92 & 0.88 & 0.86 & 0.87 & 0.83 & 1.00 \\
\hline average & 0.81 & 0.81 & 0.77 & 0.67 & 0.76 & 0.63 & 0.69 & 0.80 & 0.82 & 0.82 & 0.79 & 0.59 & 0.76 & 0.77 & 0.78 & 0.82 & 0.77 & 0.81 & 0.83 & 0.82 & 0.82 & 0.85 & 0.85 & 0.82 & 0.82 & 0.81 & 0.78 & 0.79 & 0.75 & 0.88 \\
\hline
\end{tabular}


Table A4: Pairwise correlation between measures of Breakdown

a) core indicators

\begin{tabular}{|c|c|c|c|c|c|c|c|c|c|c|c|}
\hline & 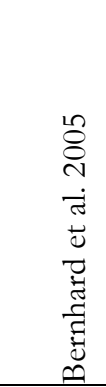 & 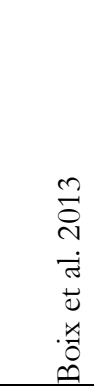 & 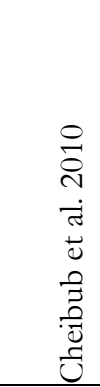 & 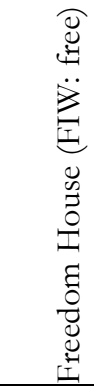 & 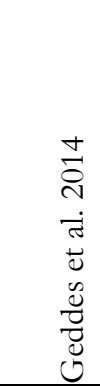 & 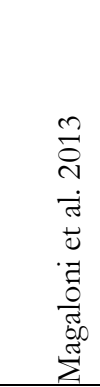 & 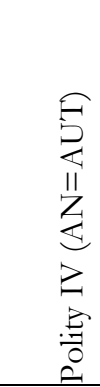 & 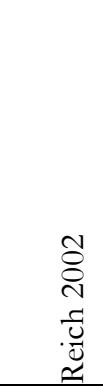 & 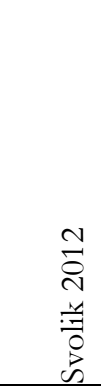 & 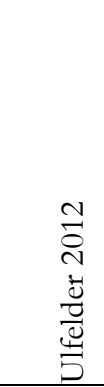 & 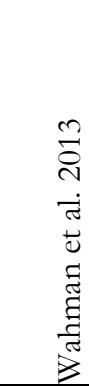 \\
\hline Bernhard et al. 2005 & 1.00 & & & & & & & & & & \\
\hline Boix et al. 2013 & 0.56 & 1.00 & & & & & & & & & \\
\hline Cheibub et al. 2010 & 0.53 & 0.77 & 1.00 & & & & & & & & \\
\hline Freedom House (FIW: free) & 0.24 & 0.23 & 0.21 & 1.00 & & & & & & & \\
\hline Geddes et al. 2014 & 0.64 & 0.54 & 0.64 & 0.12 & 1.00 & & & & & & \\
\hline Magaloni et al. 2013 & 0.57 & 0.59 & 0.66 & 0.24 & 0.58 & 1.00 & & & & & \\
\hline Polity IV (AN=AUT) & 0.57 & 0.40 & 0.33 & 0.19 & 0.39 & 0.54 & 1.00 & & & & \\
\hline Reich 2002 & 0.82 & 0.43 & 0.59 & 0.24 & 0.57 & 0.54 & 0.37 & 1.00 & & & \\
\hline Svolik 2012 & 0.82 & 0.43 & 0.59 & 0.24 & 0.57 & 0.54 & 0.37 & 0.55 & 1.00 & & \\
\hline Ulfelder 2012 & 0.60 & 0.55 & 0.58 & 0.17 & 0.60 & 0.52 & 0.44 & 0.63 & 0.60 & 1.00 & \\
\hline Wahman et al. 2013 & 0.28 & 0.22 & 0.20 & 0.29 & 0.14 & 0.26 & 0.32 & 0.17 & 0.24 & 0.16 & 1.00 \\
\hline average & 0.56 & 0.47 & 0.51 & 0.22 & 0.48 & 0.50 & 0.39 & 0.49 & 0.50 & 0.49 & 0.23 \\
\hline
\end{tabular}


b) extended set of indicators

\begin{tabular}{|c|c|c|c|c|c|c|c|c|c|c|c|c|c|c|c|c|c|c|c|c|c|c|c|c|c|c|c|}
\hline & 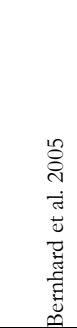 & 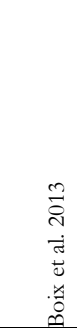 & 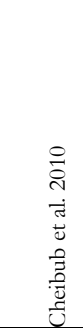 & 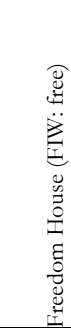 & 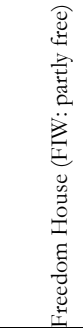 & 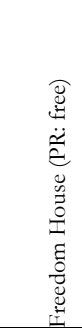 & 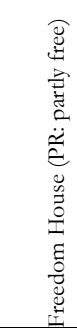 & 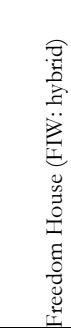 & 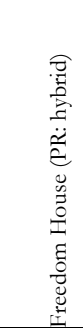 & 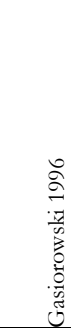 & 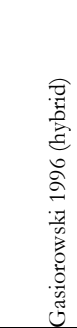 & 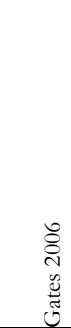 & 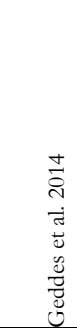 & 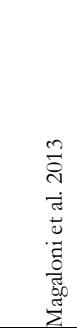 & 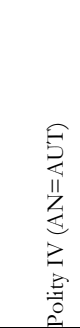 & 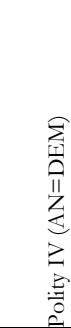 & 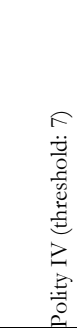 & 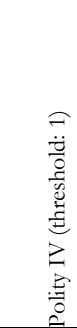 & 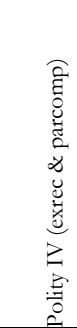 & 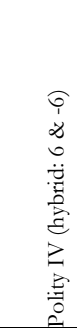 & 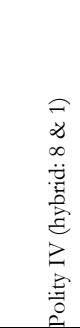 & 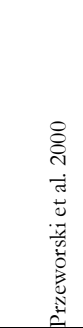 & 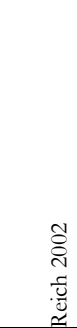 & 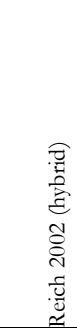 & 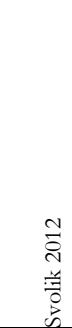 & 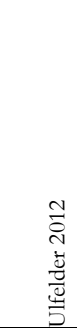 & 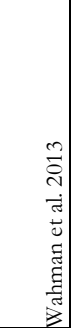 \\
\hline Bernhard et al. 2005 & 1.00 & & & & & & & & & & & & & & & & & & & & & & & & & & \\
\hline Boix et al. 2013 & 0.56 & 1.00 & & & & & & & & & & & & & & & & & & & & & & & & & \\
\hline Cheibub et al. 2010 & 0.53 & 0.77 & 1.00 & & & & & & & & & & & & & & & & & & & & & & & & \\
\hline Freedom House (FIW: free) & 0.24 & 0.23 & 0.21 & 1.00 & & & & & & & & & & & & & & & & & & & & & & & \\
\hline Freedom House (FIW: partly free) & 0.33 & 0.18 & 0.22 & 0.06 & 1.00 & & & & & & & & & & & & & & & & & & & & & & \\
\hline Freedom House (PR: free) & 0.19 & 0.26 & 0.24 & 0.66 & 0.07 & 1.00 & & & & & & & & & & & & & & & & & & & & & \\
\hline Freedom House (PR: partly free) & 0.42 & 0.24 & 0.30 & 0.11 & 0.56 & 0.11 & 1.00 & & & & & & & & & & & & & & & & & & & & \\
\hline Freedom House (FIW: hybrid) & 0.21 & 0.34 & 0.28 & 0.31 & 0.23 & 0.30 & 0.22 & 1.00 & & & & & & & & & & & & & & & & & & & \\
\hline Freedom House (PR: hybrid) & 0.33 & 0.39 & 0.35 & 0.39 & 0.19 & 0.40 & 0.30 & 0.73 & 1.00 & & & & & & & & & & & & & & & & & & \\
\hline Gasiorowski 1996 & 0.92 & 0.45 & 0.58 & 0.33 & 0.15 & 0.31 & 0.34 & 0.22 & 0.42 & 1.00 & & & & & & & & & & & & & & & & & \\
\hline Gasiorowski 1996 (hybrid) & 0.82 & 0.35 & 0.38 & 0.37 & 0.09 & 0.28 & 0.17 & 0.35 & 0.40 & 0.66 & 1.00 & & & & & & & & & & & & & & & & \\
\hline Gates 2006 & 0.59 & 0.54 & 0.52 & 0.22 & 0.20 & 0.27 & 0.32 & 0.32 & 0.49 & 0.48 & 0.34 & 1.00 & & & & & & & & & & & & & & & \\
\hline Geddes et al. 2014 & 0.64 & 0.54 & 0.64 & 0.12 & 0.24 & 0.13 & 0.29 & 0.24 & 0.31 & 0.56 & 0.43 & 0.51 & 1.00 & & & & & & & & & & & & & & \\
\hline Magaloni et al. 2013 & 0.57 & 0.59 & 0.66 & 0.24 & 0.22 & 0.27 & 0.29 & 0.44 & 0.44 & 0.53 & 0.43 & 0.58 & 0.58 & 1.00 & & & & & & & & & & & & & \\
\hline Polity IV (AN=AUT) & 0.57 & 0.40 & 0.33 & 0.19 & 0.17 & 0.17 & 0.25 & 0.29 & 0.35 & 0.40 & 0.29 & 0.50 & 0.39 & 0.54 & 1.00 & & & & & & & & & & & & \\
\hline Polity IV (AN=DEM) & 0.54 & 0.30 & 0.36 & 0.10 & 0.23 & 0.11 & 0.27 & 0.27 & 0.29 & 0.31 & 0.21 & 0.42 & 0.34 & 0.32 & 0.23 & 1.00 & & & & & & & & & & & \\
\hline Polity IV (threshold: 7) & 0.46 & 0.35 & 0.25 & 0.18 & 0.15 & 0.17 & 0.20 & 0.21 & 0.21 & 0.38 & 0.25 & 0.43 & 0.33 & 0.49 & 0.75 & 0.18 & 1.00 & & & & & & & & & & \\
\hline Polity IV (threshold: 1) & 0.63 & 0.43 & 0.51 & 0.23 & 0.25 & 0.23 & 0.35 & 0.36 & 0.43 & 0.47 & 0.37 & 0.66 & 0.53 & 0.57 & 0.46 & 0.38 & 0.41 & 1.00 & & & & & & & & & \\
\hline Polity IV (exrec \& parcomp) & 0.61 & 0.46 & 0.50 & 0.22 & 0.25 & 0.22 & 0.38 & 0.38 & 0.49 & 0.47 & 0.33 & 0.60 & 0.51 & 0.63 & 0.57 & 0.48 & 0.49 & 0.82 & 1.00 & & & & & & & & \\
\hline Polity IV (hybrid: $6 \&$-6) & 0.44 & 0.43 & 0.44 & 0.14 & 0.23 & 0.17 & 0.25 & 0.44 & 0.42 & 0.37 & 0.23 & 0.51 & 0.41 & 0.51 & 0.57 & 0.40 & 0.45 & 0.43 & 0.51 & 1.00 & & & & & & & \\
\hline Polity IV (hybrid: 8 \& 1) & 0.44 & 0.31 & 0.22 & 0.16 & 0.08 & 0.14 & 0.16 & 0.12 & 0.18 & 0.33 & 0.25 & 0.41 & 0.26 & 0.39 & 0.55 & 0.16 & 0.62 & 0.42 & 0.47 & 0.40 & 1.00 & & & & & & \\
\hline Przeworski et al. 2000 & 0.50 & 0.80 & 0.96 & 0.22 & 0.20 & 0.26 & 0.26 & 0.31 & 0.33 & 0.55 & 0.36 & 0.52 & 0.64 & 0.65 & 0.30 & 0.35 & 0.24 & 0.46 & 0.45 & 0.42 & 0.18 & 1.00 & & & & & \\
\hline Reich 2002 & 0.82 & 0.43 & 0.59 & 0.24 & 0.15 & 0.22 & 0.33 & 0.20 & 0.38 & 1.00 & 0.66 & 0.47 & 0.57 & 0.54 & 0.37 & 0.33 & 0.33 & 0.45 & 0.46 & 0.41 & 0.31 & 0.57 & 1.00 & & & & \\
\hline Reich 2002 (hybrid) & 0.72 & 0.33 & 0.38 & 0.28 & 0.11 & 0.21 & 0.19 & 0.33 & 0.38 & 0.66 & 1.00 & 0.34 & 0.43 & 0.45 & 0.28 & 0.25 & 0.24 & 0.36 & 0.35 & 0.31 & 0.26 & 0.36 & 0.66 & 1.00 & & & \\
\hline Svolik 2012 & 0.52 & 0.63 & 0.71 & 0.25 & 0.23 & 0.26 & 0.34 & 0.32 & 0.45 & 0.54 & 0.40 & 0.56 & 0.60 & 0.66 & 0.38 & 0.34 & 0.34 & 0.53 & 0.55 & 0.38 & 0.29 & 0.69 & 0.55 & 0.43 & 1.00 & & \\
\hline Ulfelder 2012 & 0.60 & 0.55 & 0.58 & 0.17 & 0.14 & 0.15 & 0.26 & 0.16 & 0.31 & 0.67 & 0.49 & 0.66 & 0.60 & 0.52 & 0.44 & 0.36 & 0.36 & 0.60 & 0.61 & 0.36 & 0.28 & 0.55 & 0.63 & 0.46 & 0.60 & 1.00 & \\
\hline Wahman et al. 2013 & 0.28 & 0.22 & 0.20 & 0.29 & 0.07 & 0.35 & 0.12 & 0.26 & 0.36 & 0.28 & 0.27 & 0.23 & 0.14 & 0.26 & 0.32 & 0.11 & 0.28 & 0.24 & 0.23 & 0.19 & 0.21 & 0.18 & 0.17 & 0.17 & 0.24 & 0.16 & 1.00 \\
\hline average & 0.52 & 0.43 & 0.45 & 0.24 & 0.19 & 0.24 & 0.27 & 0.30 & 0.37 & 0.48 & 0.39 & 0.45 & 0.42 & 0.48 & 0.39 & 0.29 & 0.34 & 0.45 & 0.46 & 0.38 & 0.29 & 0.44 & 0.46 & 0.38 & 0.47 & 0.43 & 0.23 \\
\hline
\end{tabular}


Table A5: Pairwise correlation between measures of Backsliding

a) core indicators-V-Dem continuous

\begin{tabular}{|c|c|c|c|c|c|}
\hline & 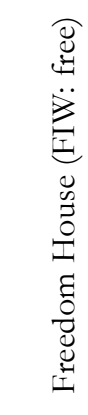 & $\begin{array}{l}\text { Z } \\
\text { : } \\
0 \\
0\end{array}$ & 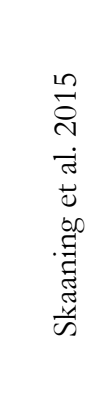 & 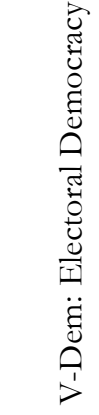 & 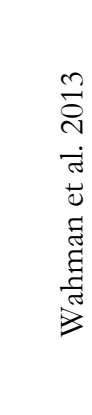 \\
\hline Freedom House (FIW: free) & 1.00 & & & & \\
\hline Polity IV & 0.27 & 1.00 & & & \\
\hline Skaaning et al. 2015 & 0.22 & 0.31 & 1.00 & & \\
\hline V-Dem: Electoral Democracy & 0.19 & 0.28 & 0.29 & 1.00 & \\
\hline Wahman et al. 2013 & 0.87 & 0.46 & 0.23 & 0.18 & 1.00 \\
\hline average & 0.39 & 0.33 & 0.26 & 0.23 & 0.43 \\
\hline
\end{tabular}


b) core indicators-V-Dem ordinal

\begin{tabular}{|c|c|c|c|c|c|}
\hline & 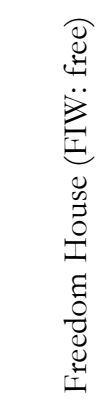 & $\begin{array}{l}Z \\
0 \\
0 \\
0\end{array}$ & 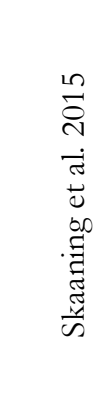 & 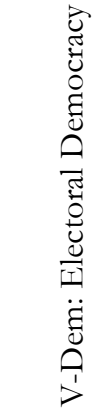 & 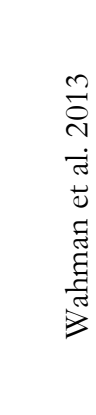 \\
\hline Freedom House (FIW: free) & 1.00 & & & & \\
\hline Polity IV & 0.27 & 1.00 & & & \\
\hline Skaaning et al. 2015 & 0.22 & 0.31 & 1.00 & & \\
\hline V-Dem: Electoral Democracy (ordinal) & 0.12 & 0.23 & 0.26 & 1.00 & \\
\hline Wahman et al. 2013 & 0.87 & 0.46 & 0.23 & 0.12 & 1.00 \\
\hline average & 0.37 & 0.32 & 0.26 & 0.18 & 0.42 \\
\hline
\end{tabular}


c) extended set of indicators-V-Dem continuous

\begin{tabular}{|c|c|c|c|c|c|c|c|c|c|c|c|c|c|c|c|c|c|c|}
\hline & 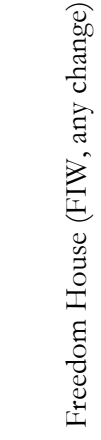 & 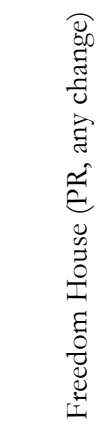 & 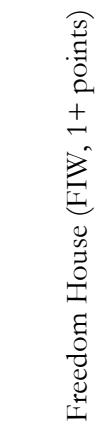 & 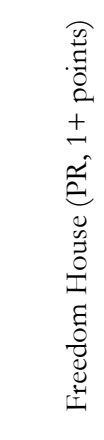 & 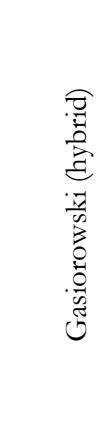 & 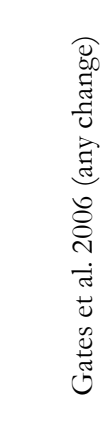 & 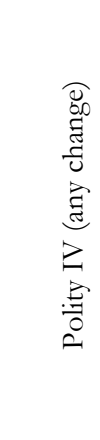 & 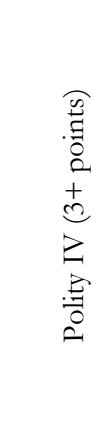 & 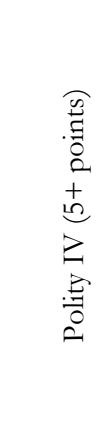 & 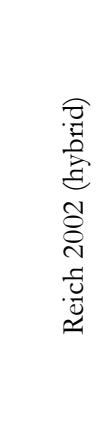 & 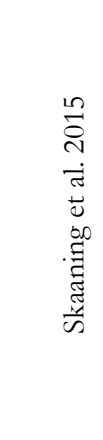 & 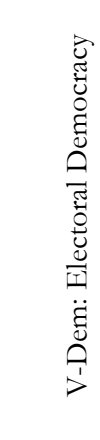 & 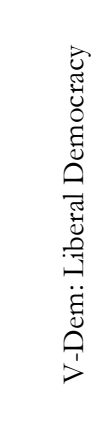 & 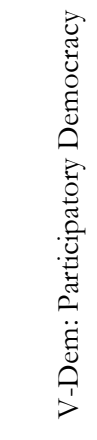 & 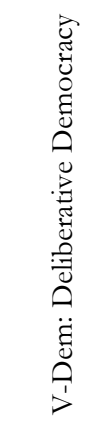 & 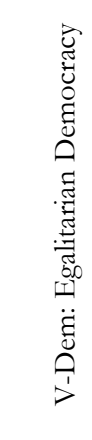 & 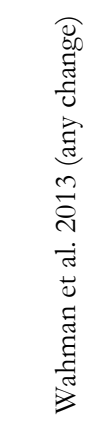 & 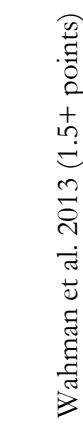 \\
\hline Freedom House (FIW, any change) & 1.00 & & & & & & & & & & & & & & & & & \\
\hline Freedom House (PR, any change) & 0.73 & 1.00 & & & & & & & & & & & & & & & & \\
\hline Freedom House (FIW, $1+$ points) & 0.47 & 0.57 & 1.00 & & & & & & & & & & & & & & & \\
\hline Freedom House (PR, $1+$ points) & 0.71 & 0.98 & 0.58 & 1.00 & & & & & & & & & & & & & & \\
\hline Gasiorowski (hybrid) & 0.15 & 0.20 & 0.22 & 0.16 & 1.00 & & & & & & & & & & & & & \\
\hline Gates et al. 2006 (any change) & 0.25 & 0.31 & 0.32 & 0.30 & 0.25 & 1.00 & & & & & & & & & & & & \\
\hline Polity IV (any change) & 0.27 & 0.30 & 0.35 & 0.30 & 0.22 & 0.53 & 1.00 & & & & & & & & & & & \\
\hline Polity IV (3+ points) & 0.27 & 0.32 & 0.37 & 0.32 & 0.27 & 0.56 & 0.72 & 1.00 & & & & & & & & & & \\
\hline Polity IV ( $5+$ points $)$ & 0.24 & 0.29 & 0.36 & 0.30 & 0.30 & 0.52 & 0.55 & 0.76 & 1.00 & & & & & & & & & \\
\hline Reich 2002 (hybrid) & 0.14 & 0.19 & 0.20 & 0.16 & 0.99 & 0.23 & 0.21 & 0.26 & 0.29 & 1.00 & & & & & & & & \\
\hline Skaaning et al. 2015 & 0.22 & 0.30 & 0.33 & 0.30 & 0.25 & 0.40 & 0.31 & 0.35 & 0.35 & 0.24 & 1.00 & & & & & & & \\
\hline V-Dem: Electoral Democracy & 0.19 & 0.20 & 0.21 & 0.20 & 0.16 & 0.24 & 0.28 & 0.30 & 0.29 & 0.16 & 0.29 & 1.00 & & & & & & \\
\hline V-Dem: Liberal Democracy & 0.18 & 0.19 & 0.21 & 0.19 & 0.18 & 0.26 & 0.28 & 0.31 & 0.30 & 0.17 & 0.24 & 0.68 & 1.00 & & & & & \\
\hline V-Dem: Participatory Democracy & 0.14 & 0.17 & 0.20 & 0.17 & 0.21 & 0.25 & 0.27 & 0.31 & 0.29 & 0.19 & 0.25 & 0.61 & 0.66 & 1.00 & & & & \\
\hline V-Dem: Deliberative Democracy & 0.14 & 0.16 & 0.19 & 0.16 & 0.17 & 0.22 & 0.24 & 0.26 & 0.26 & 0.15 & 0.25 & 0.65 & 0.66 & 0.59 & 1.00 & & & \\
\hline V-Dem: Egalitarian Democracy & 0.17 & 0.20 & 0.20 & 0.20 & 0.18 & 0.25 & 0.25 & 0.29 & 0.29 & 0.17 & 0.25 & 0.70 & 0.76 & 0.67 & 0.69 & 1.00 & & \\
\hline Wahman et al. 2013 (any change) & 0.87 & 0.65 & 0.44 & 0.65 & 0.17 & 0.27 & 0.46 & 0.31 & 0.27 & 0.16 & 0.23 & 0.18 & 0.18 & 0.15 & 0.16 & 0.17 & 1.00 & \\
\hline Wahman et al. 2013 (1.5+ points) & 0.30 & 0.37 & 0.50 & 0.38 & 0.34 & 0.50 & 0.57 & 0.79 & 0.81 & 0.29 & 0.43 & 0.31 & 0.32 & 0.30 & 0.27 & 0.33 & 0.33 & 1.00 \\
\hline average & 0.32 & 0.36 & 0.34 & 0.36 & 0.26 & 0.33 & 0.36 & 0.40 & 0.38 & 0.25 & 0.29 & 0.33 & 0.34 & 0.32 & 0.31 & 0.34 & 0.33 & 0.42 \\
\hline
\end{tabular}


d) extended set of indicators-V-Dem ordinal

\begin{tabular}{|c|c|c|c|c|c|c|c|c|c|c|c|c|c|c|c|c|c|c|}
\hline & 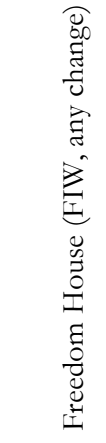 & 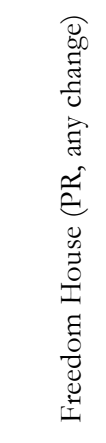 & 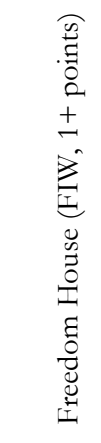 & 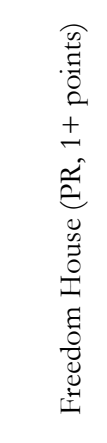 & 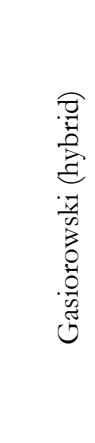 & 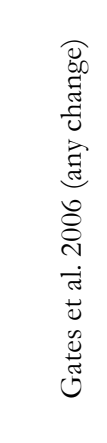 & 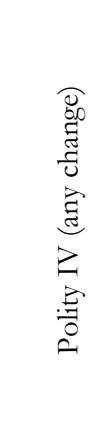 & 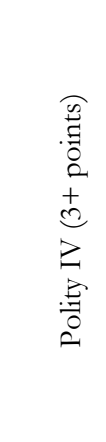 & 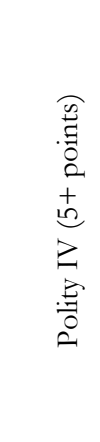 & 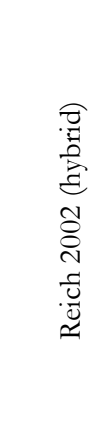 & 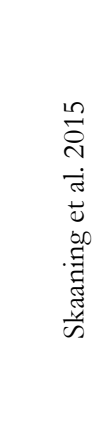 & 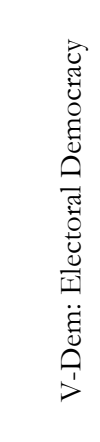 & 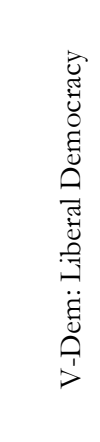 & 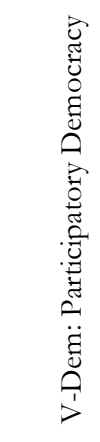 & 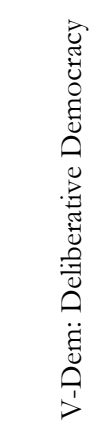 & 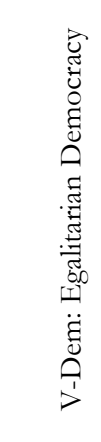 & 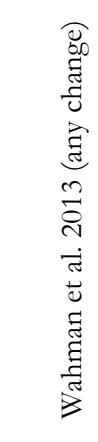 & 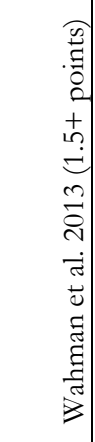 \\
\hline Freedom House (FIW, any change) & 1.00 & & & & & & & & & & & & & & & & & \\
\hline Freedom House (PR, any change) & 0.73 & 1.00 & & & & & & & & & & & & & & & & \\
\hline Freedom House (FIW, 1+ points) & 0.47 & 0.57 & 1.00 & & & & & & & & & & & & & & & \\
\hline Freedom House (PR, 1+ points) & 0.71 & 0.98 & 0.58 & 1.00 & & & & & & & & & & & & & & \\
\hline Gasiorowski (hybrid) & 0.15 & 0.20 & 0.22 & 0.16 & 1.00 & & & & & & & & & & & & & \\
\hline Gates et al. 2006 (any change) & 0.25 & 0.31 & 0.32 & 0.30 & 0.25 & 1.00 & & & & & & & & & & & & \\
\hline Polity IV (any change) & 0.27 & 0.30 & 0.35 & 0.30 & 0.22 & 0.53 & 1.00 & & & & & & & & & & & \\
\hline Polity IV (3+ points) & 0.27 & 0.32 & 0.37 & 0.32 & 0.27 & 0.56 & 0.72 & 1.00 & & & & & & & & & & \\
\hline Polity IV (5+ points) & 0.24 & 0.29 & 0.36 & 0.30 & 0.30 & 0.52 & 0.55 & 0.76 & 1.00 & & & & & & & & & \\
\hline Reich 2002 (hybrid) & 0.14 & 0.19 & 0.20 & 0.16 & 0.99 & 0.23 & 0.21 & 0.26 & 0.29 & 1.00 & & & & & & & & \\
\hline Skaaning et al. 2015 & 0.22 & 0.30 & 0.33 & 0.30 & 0.25 & 0.40 & 0.31 & 0.35 & 0.35 & 0.24 & 1.00 & & & & & & & \\
\hline V-Dem: Electoral D. (ordinal) & 0.12 & 0.16 & 0.20 & 0.16 & 0.14 & 0.21 & 0.23 & 0.25 & 0.24 & 0.14 & 0.26 & 1.00 & & & & & & \\
\hline V-Dem: Liberal D. (ordinal) & 0.12 & 0.15 & 0.19 & 0.15 & 0.21 & 0.27 & 0.24 & 0.30 & 0.33 & 0.19 & 0.25 & 0.38 & 1.00 & & & & & \\
\hline V-Dem: Participatory D. (ordinal) & 0.10 & 0.10 & 0.15 & 0.11 & 0.16 & 0.17 & 0.19 & 0.21 & 0.22 & 0.15 & 0.17 & 0.29 & 0.41 & 1.00 & & & & \\
\hline V-Dem: Deliberative D. (ordinal) & 0.09 & 0.12 & 0.15 & 0.12 & 0.16 & 0.16 & 0.17 & 0.21 & 0.24 & 0.12 & 0.18 & 0.34 & 0.38 & 0.36 & 1.00 & & & \\
\hline V-Dem: Egalitarian D. (ordinal) & 0.08 & 0.09 & 0.09 & 0.09 & 0.16 & 0.14 & 0.14 & 0.17 & 0.18 & 0.13 & 0.15 & 0.27 & 0.33 & 0.28 & 0.29 & 1.00 & & \\
\hline Wahman et al. 2013 (any change) & 0.87 & 0.65 & 0.44 & 0.65 & 0.17 & 0.27 & 0.46 & 0.31 & 0.27 & 0.16 & 0.23 & 0.12 & 0.14 & 0.13 & 0.11 & 0.10 & 1.00 & \\
\hline Wahman et al. 2013 (1.5+ points) & 0.30 & 0.37 & 0.50 & 0.38 & 0.34 & 0.50 & 0.57 & 0.79 & 0.81 & 0.29 & 0.43 & 0.25 & 0.33 & 0.28 & 0.27 & 0.16 & 0.33 & 1.00 \\
\hline average & 0.30 & 0.34 & 0.32 & 0.34 & 0.26 & 0.32 & 0.34 & 0.38 & 0.37 & 0.24 & 0.28 & 0.22 & 0.26 & 0.20 & 0.20 & 0.17 & 0.32 & 0.41 \\
\hline
\end{tabular}


Table A6: Pairwise correlation between measures of Transition

a) core indicators

\begin{tabular}{|c|c|c|c|c|c|c|c|c|c|c|}
\hline & 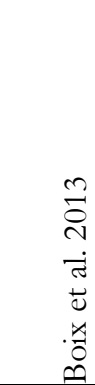 & 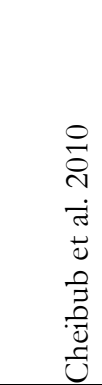 & 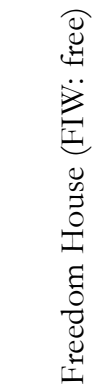 & 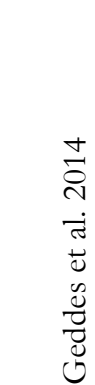 & 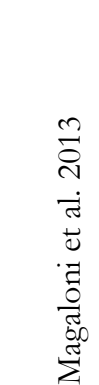 & 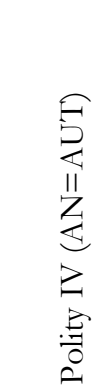 & 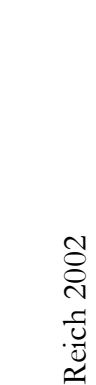 & 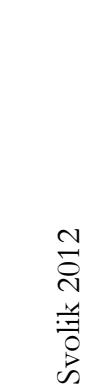 & 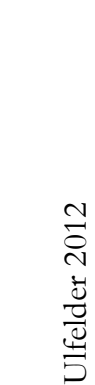 & 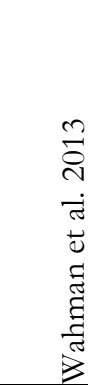 \\
\hline Boix et al. 2013 & 1.00 & & & & & & & & & \\
\hline Cheibub et al. 2010 & 0.66 & 1.00 & & & & & & & & \\
\hline Freedom House (FIW: free) & 0.33 & 0.24 & 1.00 & & & & & & & \\
\hline Geddes et al. 2014 & 0.47 & 0.60 & 0.28 & 1.00 & & & & & & \\
\hline Magaloni et al. 2013 & 0.60 & 0.63 & 0.32 & 0.59 & 1.00 & & & & & \\
\hline Polity IV (AN=AUT) & 0.37 & 0.33 & 0.25 & 0.40 & 0.54 & 1.00 & & & & \\
\hline Reich 2002 & 0.34 & 0.41 & 0.26 & 0.42 & 0.43 & 0.29 & 1.00 & & & \\
\hline Svolik 2012 & 0.62 & 0.70 & 0.33 & 0.65 & 0.67 & 0.46 & 0.49 & 1.00 & & \\
\hline Ulfelder 2012 & 0.63 & 0.63 & 0.31 & 0.63 & 0.57 & 0.42 & 0.51 & 0.70 & 1.00 & \\
\hline Wahman et al. 2013 & 0.38 & 0.28 & 0.42 & 0.34 & 0.44 & 0.55 & 0.34 & 0.42 & 0.40 & 1.00 \\
\hline average & 0.49 & 0.50 & 0.31 & 0.49 & 0.53 & 0.40 & 0.39 & 0.56 & 0.53 & 0.40 \\
\hline
\end{tabular}


b) extended set of indicators

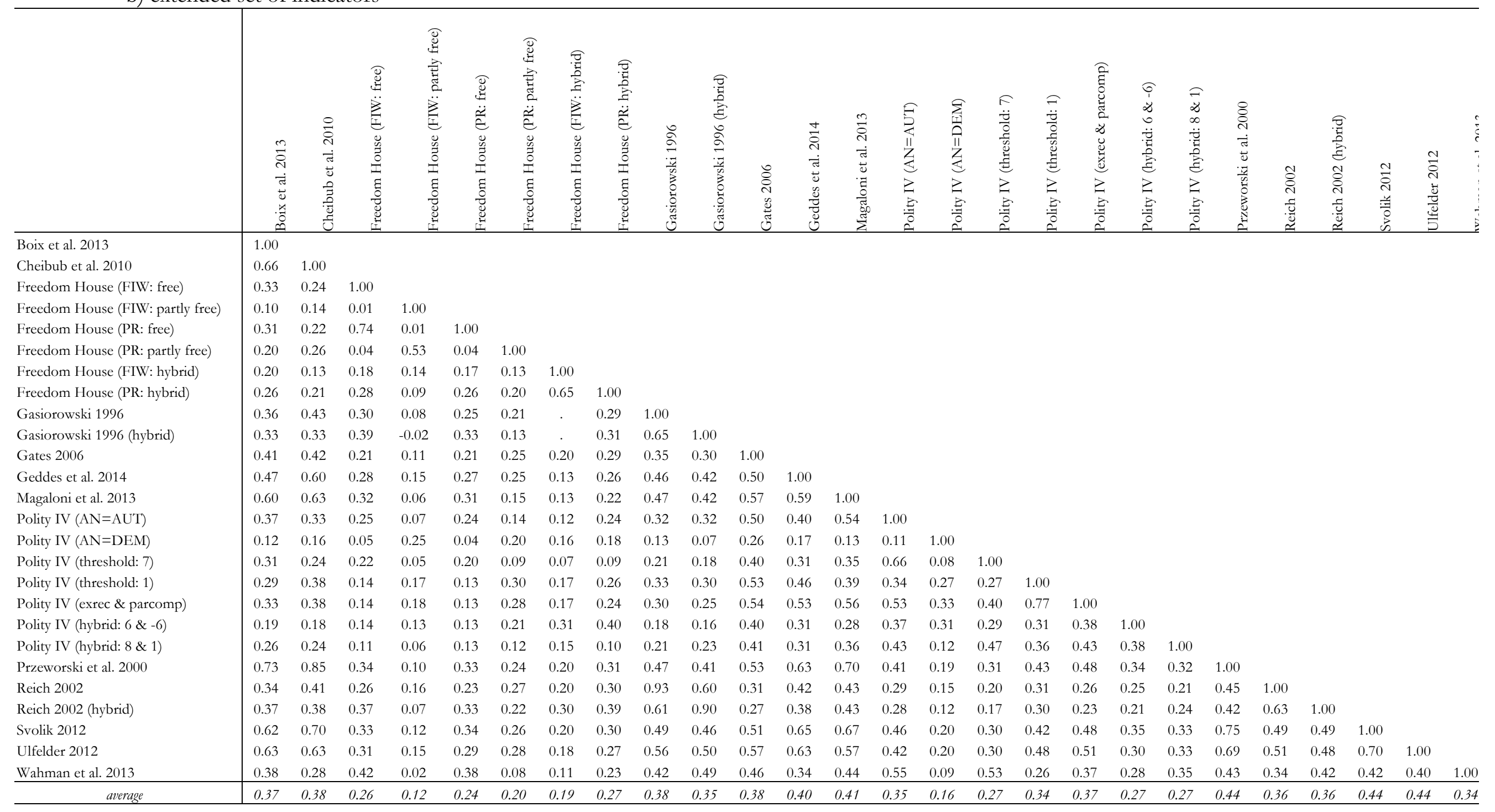


Table A7: Pairwise correlation between measures of Liberalization

a) core indicators-V-Dem continuous

\begin{tabular}{|c|c|c|c|c|c|}
\hline & 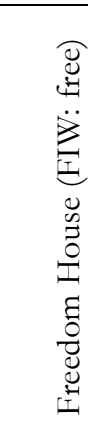 & 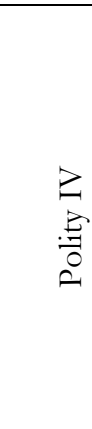 & 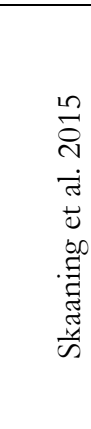 & 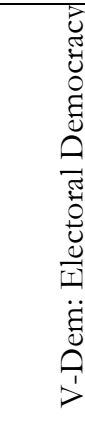 & 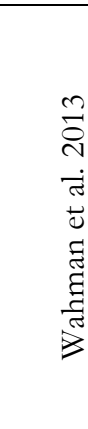 \\
\hline Freedom House (FIW: free) & 1.00 & & & & \\
\hline Polity IV & 0.33 & 1.00 & & & \\
\hline Skaaning et al. 2015 & 0.30 & 0.28 & 1.00 & & \\
\hline V-Dem: Electoral Democracy & 0.33 & 0.30 & 0.40 & 1.00 & \\
\hline Wahman et al. 2013 & 0.86 & 0.57 & 0.31 & 0.33 & 1.00 \\
\hline average & 0.45 & 0.37 & 0.32 & 0.34 & 0.52 \\
\hline
\end{tabular}


b) core indicators-V-Dem ordinal

\begin{tabular}{|c|c|c|c|c|c|}
\hline & 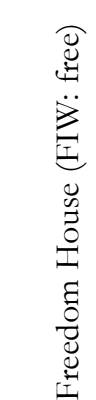 & $\begin{array}{l}Z \\
\text { 害 } \\
0\end{array}$ & 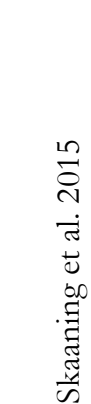 & 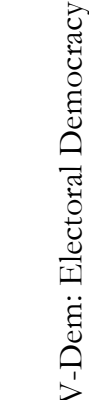 & 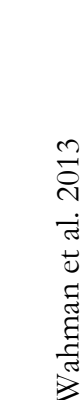 \\
\hline Freedom House (FIW: free) & 1.00 & & & & \\
\hline Polity IV & 0.33 & 1.00 & & & \\
\hline Skaaning et al. 2015 & 0.30 & 0.28 & 1.00 & & \\
\hline V-Dem: Electoral Democracy (ordinal) & 0.27 & 0.24 & 0.30 & 1.00 & \\
\hline Wahman et al. 2013 & 0.86 & 0.57 & 0.31 & 0.27 & 1.00 \\
\hline average & 0.44 & 0.36 & 0.30 & 0.27 & 0.50 \\
\hline
\end{tabular}


c) extended set of indicators-V-Dem continuous

\begin{tabular}{|c|c|c|c|c|c|c|c|c|c|c|c|c|c|c|c|c|c|c|}
\hline & 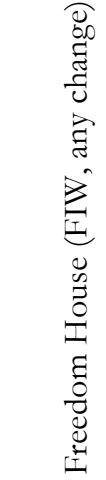 & 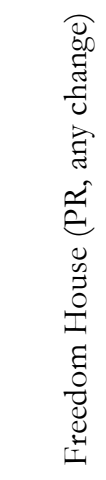 & 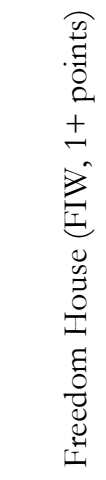 & 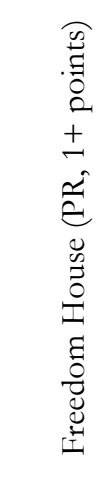 & 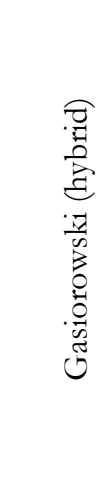 & 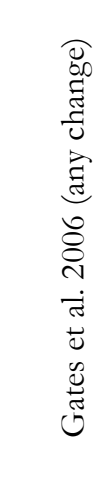 & 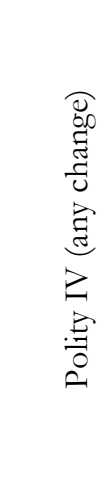 & 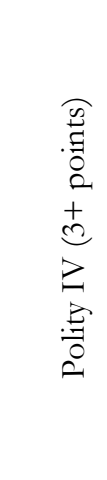 & 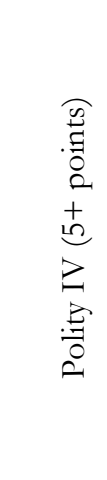 & 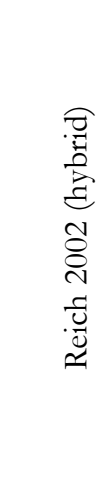 & 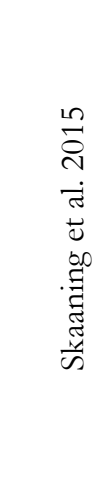 & 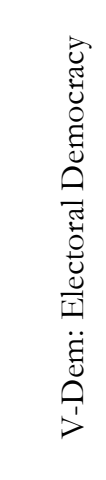 & 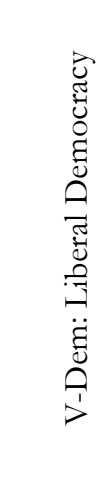 & 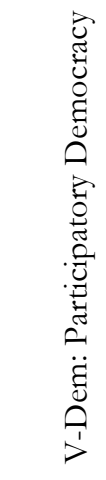 & 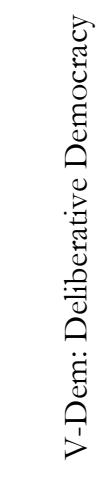 & 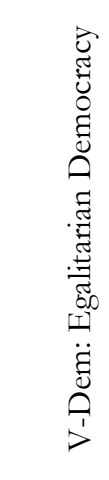 & 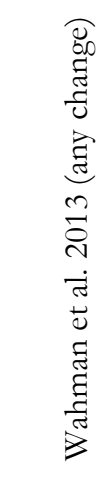 & 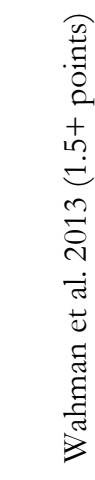 \\
\hline Freedom House (FIW, any change) & 1.00 & & & & & & & & & & & & & & & & & \\
\hline Freedom House (PR, any change) & 0.74 & 1.00 & & & & & & & & & & & & & & & & \\
\hline Freedom House (FIW, $1+$ points) & 0.49 & 0.60 & 1.00 & & & & & & & & & & & & & & & \\
\hline Freedom House (PR, $1+$ points) & 0.73 & 0.98 & 0.61 & 1.00 & & & & & & & & & & & & & & \\
\hline Gasiorowski (hybrid) & 0.16 & 0.15 & 0.16 & 0.14 & 1.00 & & & & & & & & & & & & & \\
\hline Gates et al. 2006 (any change) & 0.24 & 0.27 & 0.31 & 0.27 & 0.13 & 1.00 & & & & & & & & & & & & \\
\hline Polity IV (any change) & 0.33 & 0.33 & 0.34 & 0.33 & 0.15 & 0.46 & 1.00 & & & & & & & & & & & \\
\hline Polity IV ( $3+$ points $)$ & 0.30 & 0.33 & 0.40 & 0.33 & 0.15 & 0.50 & 0.66 & 1.00 & & & & & & & & & & \\
\hline Polity IV (5+ points) & 0.26 & 0.28 & 0.37 & 0.28 & 0.14 & 0.43 & 0.51 & 0.77 & 1.00 & & & & & & & & & \\
\hline Reich 2002 (hybrid) & 0.15 & 0.16 & 0.18 & 0.16 & 0.90 & 0.15 & 0.18 & 0.15 & 0.15 & 1.00 & & & & & & & & \\
\hline Skaaning et al. 2015 & 0.30 & 0.36 & 0.39 & 0.37 & 0.18 & 0.31 & 0.28 & 0.29 & 0.24 & 0.18 & 1.00 & & & & & & & \\
\hline V-Dem: Electoral Democracy & 0.33 & 0.36 & 0.38 & 0.36 & 0.16 & 0.28 & 0.30 & 0.31 & 0.27 & 0.16 & 0.40 & 1.00 & & & & & & \\
\hline V-Dem: Liberal Democracy & 0.31 & 0.32 & 0.38 & 0.33 & 0.20 & 0.24 & 0.28 & 0.30 & 0.27 & 0.18 & 0.32 & 0.71 & 1.00 & & & & & \\
\hline V-Dem: Participatory Democracy & 0.31 & 0.32 & 0.37 & 0.32 & 0.16 & 0.24 & 0.29 & 0.31 & 0.28 & 0.16 & 0.29 & 0.67 & 0.71 & 1.00 & & & & \\
\hline V-Dem: Deliberative Democracy & 0.27 & 0.28 & 0.31 & 0.28 & 0.17 & 0.21 & 0.26 & 0.25 & 0.23 & 0.17 & 0.29 & 0.66 & 0.71 & 0.65 & 1.00 & & & \\
\hline V-Dem: Egalitarian Democracy & 0.28 & 0.30 & 0.34 & 0.30 & 0.17 & 0.23 & 0.27 & 0.28 & 0.26 & 0.16 & 0.29 & 0.70 & 0.77 & 0.69 & 0.68 & 1.00 & & \\
\hline Wahman et al. 2013 (any change) & 0.86 & 0.63 & 0.44 & 0.64 & 0.18 & 0.32 & 0.57 & 0.40 & 0.33 & 0.17 & 0.31 & 0.33 & 0.32 & 0.32 & 0.29 & 0.30 & 1.00 & \\
\hline Wahman et al. 2013 (1.5+ points $)$ & 0.36 & 0.41 & 0.59 & 0.42 & 0.18 & 0.50 & 0.54 & 0.77 & 0.80 & 0.17 & 0.39 & 0.41 & 0.41 & 0.42 & 0.34 & 0.37 & 0.38 & 1.00 \\
\hline average & 0.38 & 0.40 & 0.39 & 0.40 & 0.21 & 0.30 & 0.36 & 0.38 & 0.34 & 0.21 & 0.31 & 0.40 & 0.40 & 0.38 & 0.36 & 0.38 & 0.40 & 0.44 \\
\hline
\end{tabular}


d) extended set of indicators-V-Dem ordinal

\begin{tabular}{|c|c|c|c|c|c|c|c|c|c|c|c|c|c|c|c|c|c|c|}
\hline & 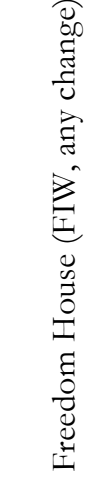 & 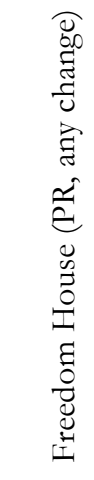 & 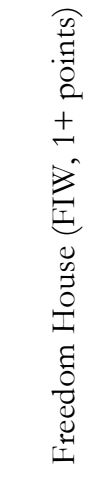 & 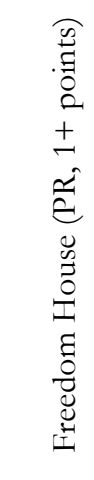 & 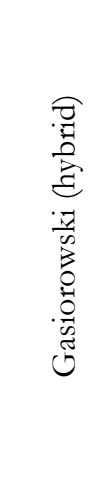 & 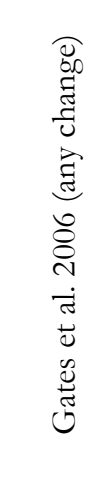 & 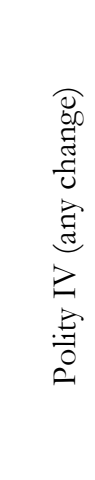 & 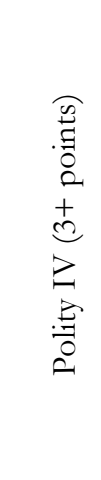 & 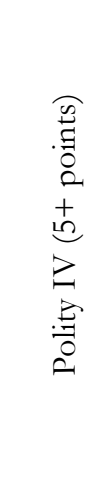 & 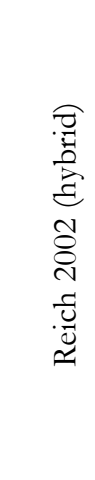 & 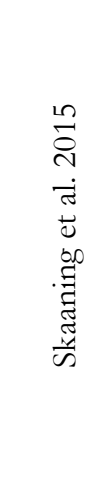 & 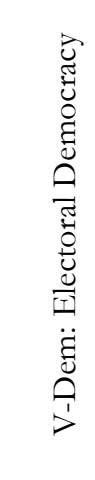 & 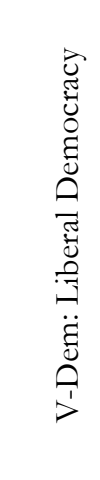 & 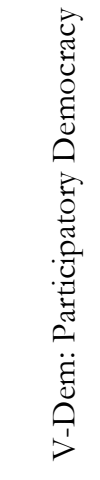 & 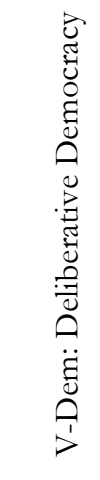 & 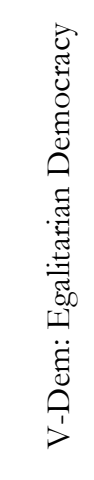 & 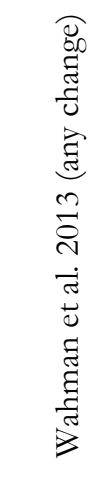 & 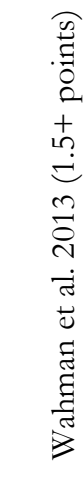 \\
\hline Freedom House (FIW, any change) & 1.00 & & & & & & & & & & & & & & & & & \\
\hline Freedom House (PR, any change) & 0.74 & 1.00 & & & & & & & & & & & & & & & & \\
\hline Freedom House (FIW, $1+$ points) & 0.49 & 0.60 & 1.00 & & & & & & & & & & & & & & & \\
\hline Freedom House (PR, $1+$ points) & 0.73 & 0.98 & 0.61 & 1.00 & & & & & & & & & & & & & & \\
\hline Gasiorowski (hybrid) & 0.16 & 0.15 & 0.16 & 0.14 & 1.00 & & & & & & & & & & & & & \\
\hline Gates et al. 2006 (any change) & 0.24 & 0.27 & 0.31 & 0.27 & 0.13 & 1.00 & & & & & & & & & & & & \\
\hline Polity IV (any change) & 0.33 & 0.33 & 0.34 & 0.33 & 0.15 & 0.46 & 1.00 & & & & & & & & & & & \\
\hline Polity IV (3+ points) & 0.30 & 0.33 & 0.40 & 0.33 & 0.15 & 0.50 & 0.66 & 1.00 & & & & & & & & & & \\
\hline Polity IV (5+ points) & 0.26 & 0.28 & 0.37 & 0.28 & 0.14 & 0.43 & 0.51 & 0.77 & 1.00 & & & & & & & & & \\
\hline Reich 2002 (hybrid) & 0.15 & 0.16 & 0.18 & 0.16 & 0.90 & 0.15 & 0.18 & 0.15 & 0.15 & 1.00 & & & & & & & & \\
\hline Skaaning et al. 2015 & 0.30 & 0.36 & 0.39 & 0.37 & 0.18 & 0.31 & 0.28 & 0.29 & 0.24 & 0.18 & 1.00 & & & & & & & \\
\hline V-Dem: Electoral D. (ordinal) & 0.27 & 0.29 & 0.31 & 0.29 & 0.15 & 0.21 & 0.24 & 0.25 & 0.23 & 0.16 & 0.30 & 1.00 & & & & & & \\
\hline V-Dem: Liberal D. (ordinal) & 0.24 & 0.25 & 0.30 & 0.25 & 0.17 & 0.15 & 0.19 & 0.22 & 0.22 & 0.12 & 0.22 & 0.40 & 1.00 & & & & & \\
\hline V-Dem: Participatory D. (ordinal) & 0.21 & 0.23 & 0.27 & 0.24 & 0.06 & 0.14 & 0.17 & 0.18 & 0.18 & 0.11 & 0.20 & 0.38 & 0.43 & 1.00 & & & & \\
\hline V-Dem: Deliberative D. (ordinal) & 0.19 & 0.23 & 0.27 & 0.23 & 0.16 & 0.13 & 0.18 & 0.18 & 0.17 & 0.15 & 0.22 & 0.40 & 0.39 & 0.42 & 1.00 & & & \\
\hline V-Dem: Egalitarian D. (ordinal) & 0.21 & 0.23 & 0.26 & 0.23 & 0.08 & 0.13 & 0.17 & 0.19 & 0.19 & 0.09 & 0.22 & 0.37 & 0.39 & 0.38 & 0.37 & 1.00 & & \\
\hline Wahman et al. 2013 (any change) & 0.86 & 0.63 & 0.44 & 0.64 & 0.18 & 0.32 & 0.57 & 0.40 & 0.33 & 0.17 & 0.31 & 0.27 & 0.22 & 0.23 & 0.21 & 0.19 & 1.00 & \\
\hline Wahman et al. 2013 (1.5+ points $)$ & 0.36 & 0.41 & 0.59 & 0.42 & 0.18 & 0.50 & 0.54 & 0.77 & 0.80 & 0.17 & 0.39 & 0.34 & 0.33 & 0.30 & 0.28 & 0.29 & 0.38 & 1.00 \\
\hline average & 0.36 & 0.38 & 0.37 & 0.38 & 0.19 & 0.27 & 0.33 & 0.36 & 0.33 & 0.19 & 0.28 & 0.29 & 0.27 & 0.24 & 0.25 & 0.23 & 0.37 & 0.41 \\
\hline
\end{tabular}


Table A8: Agreement around focal points

b) core indicators-V-Dem ordinal

\begin{tabular}{|l|ccccc|}
\hline & Mean & No coups & Coups & $\begin{array}{c}\text { No } \\
\text { Elections }\end{array}$ & Elections \\
\hline Breakdown & $28.28 \%$ & $17.53 \%$ & $50.47 \%$ & $29.05 \%$ & $25.68 \%$ \\
Backsliding & $42.96 \%$ & $40.12 \%$ & $56.68 \%$ & $42.43 \%$ & $42.38 \%$ \\
Backsliding \& Breakdown & $18.50 \%$ & $15.36 \%$ & $34.06 \%$ & $17.71 \%$ & $19.29 \%$ \\
\hline Transition & $29.87 \%$ & $29.32 \%$ & $35.53 \%$ & $17.94 \%$ & $35.01 \%$ \\
Liberalization & $48.86 \%$ & $48.67 \%$ & $51.67 \%$ & $42.16 \%$ & $53.45 \%$ \\
Liberalization \& Transition & $21.73 \%$ & $21.53 \%$ & $24.49 \%$ & $16.51 \%$ & $27.27 \%$ \\
\hline
\end{tabular}

c) extended set of indicators-V-Dem continuous

\begin{tabular}{|l|ccccc|}
\hline & Mean & No coups & Coups & $\begin{array}{c}\text { No } \\
\text { Elections }\end{array}$ & Elections \\
\hline Breakdown & $15.03 \%$ & $9.17 \%$ & $33.36 \%$ & $14.64 \%$ & $15.27 \%$ \\
Backsliding & $23.91 \%$ & $20.79 \%$ & $40.88 \%$ & $23.75 \%$ & $22.59 \%$ \\
Backsliding \& Breakdown & $12.70 \%$ & $9.97 \%$ & $27.62 \%$ & $12.42 \%$ & $12.68 \%$ \\
\hline Transition & $14.65 \%$ & $14.37 \%$ & $17.38 \%$ & $8.75 \%$ & $18.92 \%$ \\
Liberalization & $28.65 \%$ & $28.55 \%$ & $30.32 \%$ & $22.62 \%$ & $33.15 \%$ \\
Liberalization \& Transition & $15.25 \%$ & $15.08 \%$ & $17.84 \%$ & $11.58 \%$ & $19.21 \%$ \\
\hline
\end{tabular}

d) extended set of indicators-V-Dem ordinal

\begin{tabular}{|l|ccccc|}
\hline & Mean & No coups & Coups & $\begin{array}{c}\text { No } \\
\text { Elections }\end{array}$ & Elections \\
\hline Breakdown & $15.03 \%$ & $9.17 \%$ & $33.36 \%$ & $14.64 \%$ & $15.27 \%$ \\
Backsliding & $21.06 \%$ & $18.40 \%$ & $36.07 \%$ & $20.67 \%$ & $20.21 \%$ \\
Backsliding \& Breakdown & $11.55 \%$ & $9.01 \%$ & $26.17 \%$ & $11.18 \%$ & $11.71 \%$ \\
\hline Transition & $14.65 \%$ & $14.37 \%$ & $17.38 \%$ & $8.75 \%$ & $18.92 \%$ \\
Liberalization & $23.37 \%$ & $23.25 \%$ & $25.20 \%$ & $18.45 \%$ & $27.51 \%$ \\
Liberalization \& Transition & $12.79 \%$ & $12.61 \%$ & $15.53 \%$ & $9.36 \%$ & $16.44 \%$ \\
\hline
\end{tabular}


Table A9: Agreement between regime change indicators, one-year period

b) core indicators-V-Dem ordinal

\begin{tabular}{|c|c|c|c|c|c|c|}
\hline & (1) & (2) & (3) & (4) & (5) & (6) \\
\hline & Breakdown & Backsliding & $\begin{array}{c}\text { Breakdown \& } \\
\text { Backsliding }\end{array}$ & Transition & Liberalization & $\begin{array}{c}\text { Transition \& } \\
\text { Liberalization }\end{array}$ \\
\hline \multirow[t]{2}{*}{ Successful coup in $t$} & $0.276^{* * *}$ & $0.177 * * *$ & $0.190^{* * *}$ & -0.013 & 0.037 & 0.022 \\
\hline & $(0.06)$ & $(0.02)$ & $(0.03)$ & $(0.10)$ & $(0.03)$ & $(0.02)$ \\
\hline \multirow[t]{2}{*}{ Election in $\mathrm{t}$} & 0.006 & 0.009 & 0.015 & $0.141^{* * *}$ & $0.140^{* * *}$ & $0.131 * * *$ \\
\hline & $(0.03)$ & $(0.02)$ & $(0.01)$ & $(0.04)$ & $(0.01)$ & $(0.01)$ \\
\hline \multirow[t]{2}{*}{ Population, $\ln (\varnothing \mathrm{t}-3$ to $\mathrm{t}-1)$} & -3.818 & 0.561 & -1.320 & -7.547 & 1.223 & -0.686 \\
\hline & $(4.32)$ & $(2.27)$ & $(1.11)$ & $(4.77)$ & $(1.27)$ & $(0.73)$ \\
\hline \multirow[t]{2}{*}{ GDP p.c., $\ln (\varnothing \mathrm{t}-3$ to t-1) } & -4.117 & 0.578 & -1.354 & -7.384 & 1.182 & -0.698 \\
\hline & $(4.31)$ & $(2.28)$ & $(1.12)$ & $(4.69)$ & $(1.29)$ & $(0.75)$ \\
\hline \multirow[t]{2}{*}{ GDP, $\ln (\varnothing \mathrm{t}-3$ to $\mathrm{t}-1)$} & 4.086 & -0.544 & 1.364 & 7.455 & -1.271 & 0.656 \\
\hline & $(4.32)$ & $(2.28)$ & $(1.11)$ & $(4.75)$ & $(1.29)$ & $(0.75)$ \\
\hline \multirow[t]{2}{*}{ Trade $(\varnothing \mathrm{t}-3$ to $\mathrm{t}-1)$} & -0.001 & 0.000 & -0.000 & -0.000 & -0.001 & -0.000 \\
\hline & $(0.00)$ & $(0.00)$ & $(0.00)$ & $(0.00)$ & $(0.00)$ & $(0.00)$ \\
\hline \multirow[t]{2}{*}{ Aid p.c., $\ln (\varnothing \mathrm{t}-3$ to $\mathrm{t}-1)$} & 0.000 & 0.008 & 0.006 & 0.019 & -0.016 & -0.011 \\
\hline & $(0.02)$ & $(0.01)$ & $(0.01)$ & $(0.03)$ & $(0.01)$ & $(0.01)$ \\
\hline \multirow[t]{2}{*}{ Member of GATT/WTO, t-1 } & -0.021 & 0.021 & 0.007 & 0.065 & -0.001 & 0.011 \\
\hline & $(0.07)$ & $(0.02)$ & $(0.02)$ & $(0.05)$ & $(0.03)$ & $(0.02)$ \\
\hline \multirow[t]{2}{*}{ \# measures available, $\ln$} & 0.393 & & $0.685^{* * *}$ & 0.431 & -0.950 & -0.267 \\
\hline & $(0.47)$ & & $(0.23)$ & $(0.74)$ & $(0.82)$ & $(0.20)$ \\
\hline $\mathrm{N}$ & 223 & 810 & 833 & 276 & 1011 & 1024 \\
\hline Adj. R2 & 0.456 & 0.182 & 0.229 & 0.163 & 0.133 & 0.148 \\
\hline
\end{tabular}

c) extended set of indicators-V-Dem continuous

\begin{tabular}{|c|c|c|c|c|c|c|}
\hline & $\left(1^{\prime}\right)$ & $\left(2^{\prime}\right)$ & $\left(3^{\prime}\right)$ & $\left(4^{\prime}\right)$ & $\left(5^{\prime}\right)$ & $\left(6^{\prime}\right)$ \\
\hline & Breakdown & Backsliding & $\begin{array}{c}\text { Breakdown \& } \\
\text { Backsliding }\end{array}$ & Transition & Liberalization & $\begin{array}{l}\text { Transition \& } \\
\text { Liberalization }\end{array}$ \\
\hline Successful coup in $t$ & $\begin{array}{c}0.194^{* * *} \\
(0.03)\end{array}$ & $\begin{array}{c}0.217^{* * *} * \\
(0.03)\end{array}$ & $\begin{array}{c}0.185^{* * *} \\
(0.02)\end{array}$ & $\begin{array}{c}-0.008 \\
(0.03)\end{array}$ & $\begin{array}{l}0.013 \\
(0.03)\end{array}$ & $\begin{array}{l}0.018 \\
(0.02)\end{array}$ \\
\hline Election in $\mathrm{t}$ & $\begin{array}{l}0.002 \\
(0.02)\end{array}$ & $\begin{array}{l}0.017 \\
(0.02)\end{array}$ & $\begin{array}{c}0.021 * \\
(0.01)\end{array}$ & $\begin{array}{c}0.110^{* * *} \\
(0.02)\end{array}$ & $\begin{array}{c}0.155^{* * *} \\
(0.01)\end{array}$ & $\begin{array}{c}0.111 \text { *** } \\
(0.01)\end{array}$ \\
\hline Population, $\ln (\varnothing \mathrm{t}-3$ to $\mathrm{t}-1)$ & $\begin{array}{l}0.029 \\
(4.01)\end{array}$ & $\begin{array}{l}2.210 \\
(3.22)\end{array}$ & $\begin{array}{l}0.423 \\
(1.63)\end{array}$ & $\begin{array}{c}-0.333 \\
(0.77)\end{array}$ & $\begin{array}{c}-0.892 \\
(1.22)\end{array}$ & $\begin{array}{c}-0.816 \\
(0.79)\end{array}$ \\
\hline GDP p.c., $\ln (\varnothing \mathrm{t}-3$ to $\mathrm{t}-1)$ & $\begin{array}{l}-0.191 \\
(4.01)\end{array}$ & $\begin{array}{l}2.156 \\
(3.23)\end{array}$ & $\begin{array}{l}0.366 \\
(1.63)\end{array}$ & $\begin{array}{l}-0.331 \\
(0.84)\end{array}$ & $\begin{array}{l}-0.903 \\
(1.23)\end{array}$ & $\begin{array}{c}-0.844 \\
(0.80)\end{array}$ \\
\hline GDP, $\ln (\varnothing \mathrm{t}-3$ to t-1) & $\begin{array}{l}0.207 \\
(4.01)\end{array}$ & $\begin{array}{l}-2.134 \\
(3.23)\end{array}$ & $\begin{array}{l}-0.362 \\
(1.63)\end{array}$ & $\begin{array}{l}0.325 \\
(0.86)\end{array}$ & $\begin{array}{l}0.826 \\
(1.23)\end{array}$ & $\begin{array}{l}0.793 \\
(0.80)\end{array}$ \\
\hline Trade $(\varnothing \mathrm{t}-3$ to $\mathrm{t}-1)$ & $\begin{array}{r}-0.001 \\
(0.00)\end{array}$ & $\begin{array}{r}-0.000 \\
(0.00)\end{array}$ & $\begin{array}{r}-0.000 \\
(0.00)\end{array}$ & $\begin{array}{r}-0.000 \\
(0.00)\end{array}$ & $\begin{array}{c}-0.000 \\
(0.00)\end{array}$ & $\begin{array}{c}-0.000 \\
(0.00)\end{array}$ \\
\hline Aid p.c., $\ln (\varnothing \mathrm{t}-3$ to t-1) & $\begin{array}{l}0.016 \\
(0.02)\end{array}$ & $\begin{array}{l}0.010 \\
(0.01)\end{array}$ & $\begin{array}{l}0.007 \\
(0.01)\end{array}$ & $\begin{array}{c}-0.002 \\
(0.01)\end{array}$ & $\begin{array}{c}-0.020^{* *} \\
(0.01)\end{array}$ & $\begin{array}{c}-0.013^{* *} \\
(0.01)\end{array}$ \\
\hline Member of GATT/WTO, t-1 & $\begin{array}{c}-0.041 \\
(0.04)\end{array}$ & $\begin{array}{l}0.018 \\
(0.02)\end{array}$ & $\begin{array}{l}0.006 \\
(0.02)\end{array}$ & $\begin{array}{l}0.034 \\
(0.03)\end{array}$ & $\begin{array}{l}0.013 \\
(0.03)\end{array}$ & $\begin{array}{l}0.012 \\
(0.02)\end{array}$ \\
\hline \# measures available, $\ln$ & $\begin{array}{c}0.920^{* *} \\
(0.37)\end{array}$ & $\begin{array}{c}-0.558^{* * *} \\
(0.20) \\
\end{array}$ & $\begin{array}{r}-0.027 \\
(0.18) \\
\end{array}$ & $\begin{array}{l}-0.127 \\
(0.19) \\
\end{array}$ & $\begin{array}{c}-1.317^{* * *} \\
(0.30) \\
\end{array}$ & $\begin{array}{c}-0.534^{* * *} \\
(0.15) \\
\end{array}$ \\
\hline $\begin{array}{l}\mathrm{N} \\
\text { Adj. R2 }\end{array}$ & $\begin{array}{c}381 \\
0.333\end{array}$ & $\begin{array}{c}940 \\
0.168\end{array}$ & $\begin{array}{c}969 \\
0.201\end{array}$ & $\begin{array}{c}491 \\
0.121\end{array}$ & $\begin{array}{l}1215 \\
0.190\end{array}$ & $\begin{array}{l}1233 \\
0.170\end{array}$ \\
\hline
\end{tabular}


d) extended set of indicators-V-Dem ordinal

\begin{tabular}{|c|c|c|c|c|c|c|}
\hline & $\left(1^{\prime}\right)$ & $\left(2^{\prime}\right)$ & $\left(3^{\prime}\right)$ & $\left(4^{\prime}\right)$ & $\left(5^{\prime}\right)$ & $\left(6^{\prime}\right)$ \\
\hline & Breakdown & Backsliding & $\begin{array}{c}\text { Breakdown \& } \\
\text { Backsliding }\end{array}$ & Transition & Liberalization & $\begin{array}{c}\text { Transition \& } \\
\text { Liberalization }\end{array}$ \\
\hline Successful coup in $t$ & $\begin{array}{c}0.194 * * * \\
(0.03)\end{array}$ & $\begin{array}{c}0.197 * * * \\
(0.03)\end{array}$ & $\begin{array}{c}0.178 * * * \\
(0.02)\end{array}$ & $\begin{array}{c}-0.008 \\
(0.03)\end{array}$ & $\begin{array}{c}-0.003 \\
(0.03)\end{array}$ & $\begin{array}{l}0.007 \\
(0.02)\end{array}$ \\
\hline Election in $\mathrm{t}$ & $\begin{array}{l}0.002 \\
(0.02)\end{array}$ & $\begin{array}{l}0.016 \\
(0.01)\end{array}$ & $\begin{array}{l}0.018 \\
(0.01)\end{array}$ & $\begin{array}{c}0.110^{* * *} \\
(0.02)\end{array}$ & $\begin{array}{c}0.135^{* * *} \\
(0.01)\end{array}$ & $\begin{array}{c}0.102^{* * *} \\
(0.01)\end{array}$ \\
\hline Population, $\ln (\varnothing \mathrm{t}-3$ to $\mathrm{t}-1)$ & $\begin{array}{l}0.029 \\
(4.01)\end{array}$ & $\begin{array}{l}0.763 \\
(2.51)\end{array}$ & $\begin{array}{c}-0.011 \\
(1.54)\end{array}$ & $\begin{array}{c}-0.333 \\
(0.77)\end{array}$ & $\begin{array}{r}-0.197 \\
(1.30)\end{array}$ & $\begin{array}{c}-0.564 \\
(0.98)\end{array}$ \\
\hline GDP p.c., $\ln (\varnothing \mathrm{t}-3$ to $\mathrm{t}-1)$ & $\begin{array}{r}-0.191 \\
(4.01)\end{array}$ & $\begin{array}{l}0.748 \\
(2.51)\end{array}$ & $\begin{array}{l}-0.053 \\
(1.54)\end{array}$ & $\begin{array}{l}-0.331 \\
(0.84)\end{array}$ & $\begin{array}{l}-0.250 \\
(1.31)\end{array}$ & $\begin{array}{l}-0.603 \\
(0.99)\end{array}$ \\
\hline GDP, $\ln (\varnothing \mathrm{t}-3$ to $\mathrm{t}-1)$ & $\begin{array}{l}0.207 \\
(4.01)\end{array}$ & $\begin{array}{l}-0.727 \\
(2.51)\end{array}$ & $\begin{array}{l}0.056 \\
(1.54)\end{array}$ & $\begin{array}{l}0.325 \\
(0.86)\end{array}$ & $\begin{array}{l}0.167 \\
(1.31)\end{array}$ & $\begin{array}{l}0.548 \\
(0.99)\end{array}$ \\
\hline Trade $(\varnothing \mathrm{t}-3$ to $\mathrm{t}-1)$ & $\begin{array}{l}-0.001 \\
(0.00)\end{array}$ & $\begin{array}{l}-0.000 \\
(0.00)\end{array}$ & $\begin{array}{l}-0.000 \\
(0.00)\end{array}$ & $\begin{array}{l}-0.000 \\
(0.00)\end{array}$ & $\begin{array}{l}0.000 \\
(0.00)\end{array}$ & $\begin{array}{c}-0.000 \\
(0.00)\end{array}$ \\
\hline Aid p.c., $\ln (\varnothing \mathrm{t}-3$ to $\mathrm{t}-1)$ & $\begin{array}{l}0.016 \\
(0.02)\end{array}$ & $\begin{array}{l}0.002 \\
(0.01)\end{array}$ & $\begin{array}{l}0.003 \\
(0.01)\end{array}$ & $\begin{array}{r}-0.002 \\
(0.01)\end{array}$ & $\begin{array}{c}-0.025^{* * *} \\
(0.01)\end{array}$ & $\begin{array}{c}-0.018^{* * *} \\
(0.01)\end{array}$ \\
\hline Member of GATT/WTO, t-1 & $\begin{array}{c}-0.041 \\
(0.04)\end{array}$ & $\begin{array}{l}0.016 \\
(0.02)\end{array}$ & $\begin{array}{l}0.004 \\
(0.01)\end{array}$ & $\begin{array}{l}0.034 \\
(0.03)\end{array}$ & $\begin{array}{l}0.017 \\
(0.02)\end{array}$ & $\begin{array}{l}0.014 \\
(0.02)\end{array}$ \\
\hline \# measures available, $\ln$ & $\begin{array}{c}0.920^{* *} \\
(0.37)\end{array}$ & $\begin{array}{c}-0.516^{* * *} \\
(0.14)\end{array}$ & $\begin{array}{l}0.005 \\
(0.16)\end{array}$ & $\begin{array}{l}-0.127 \\
(0.19)\end{array}$ & $\begin{array}{c}-1.256^{* * *} \\
(0.26)\end{array}$ & $\begin{array}{c}-0.529 * * * \\
(0.14)\end{array}$ \\
\hline $\begin{array}{l}\mathrm{N} \\
\text { Adj. R2 }\end{array}$ & $\begin{array}{c}381 \\
0.333\end{array}$ & $\begin{array}{c}960 \\
0.174\end{array}$ & $\begin{array}{c}988 \\
0.203\end{array}$ & $\begin{array}{c}491 \\
0.121\end{array}$ & $\begin{array}{l}1232 \\
0.180\end{array}$ & $\begin{array}{l}1251 \\
0.152\end{array}$ \\
\hline
\end{tabular}


Table A10: Agreement between regime change indicators, three-year period

a) core indicators-V-Dem continuous

\begin{tabular}{|c|c|c|c|c|c|c|}
\hline & (7) & $(8)$ & (9) & (10) & (11) & (12) \\
\hline & Breakdown & Backsliding & $\begin{array}{c}\text { Breakdown \& } \\
\text { Backsliding }\end{array}$ & Transition & Liberalization & $\begin{array}{c}\text { Transition \& } \\
\text { Liberalization }\end{array}$ \\
\hline Successful coup in $t$ & $\begin{array}{c}0.096^{* * *} \\
(0.02)\end{array}$ & $\begin{array}{c}0.155^{* * *} \\
(0.02)\end{array}$ & $\begin{array}{c}0.132^{* * *} \\
(0.02)\end{array}$ & $\begin{array}{c}0.082^{*} \\
(0.05)\end{array}$ & $\begin{array}{c}0.041 * \\
(0.02)\end{array}$ & $\begin{array}{c}0.063 * * * \\
(0.02)\end{array}$ \\
\hline Election in $\mathrm{t}$ & $\begin{array}{l}0.006 \\
(0.02)\end{array}$ & $\begin{array}{l}0.008 \\
(0.01)\end{array}$ & $\begin{array}{l}0.004 \\
(0.01)\end{array}$ & $\begin{array}{c}0.036^{* *} \\
(0.01)\end{array}$ & $\begin{array}{c}0.071 * * * \\
(0.01)\end{array}$ & $\begin{array}{c}0.056^{* * *} \\
(0.01)\end{array}$ \\
\hline Population, $\ln (\varnothing \mathrm{t}-3$ to $\mathrm{t}-1)$ & $\begin{array}{l}0.315 \\
(2.15)\end{array}$ & $\begin{array}{l}0.567 \\
(1.17)\end{array}$ & $\begin{array}{c}-2.748^{* *} \\
(1.10)\end{array}$ & $\begin{array}{c}-5.544^{*} \\
(2.81)\end{array}$ & $\begin{array}{l}-1.376 \\
(1.36)\end{array}$ & $\begin{array}{c}-2.126^{* *} \\
(0.97)\end{array}$ \\
\hline GDP p.c., $\ln (\varnothing \mathrm{t}-3$ to t-1) & $\begin{array}{l}0.009 \\
(2.17)\end{array}$ & $\begin{array}{l}0.426 \\
(1.19)\end{array}$ & $\begin{array}{c}-2.941 * * * \\
(1.11)\end{array}$ & $\begin{array}{c}-5.374^{*} \\
(2.87)\end{array}$ & $\begin{array}{l}-1.497 \\
(1.38)\end{array}$ & $\begin{array}{c}-2.207^{* *} \\
(0.98)\end{array}$ \\
\hline $\mathrm{GDP}, \ln (\varnothing \mathrm{t}-3$ to $\mathrm{t}-1)$ & $\begin{array}{l}-0.109 \\
(2.17)\end{array}$ & $\begin{array}{l}-0.421 \\
(1.18)\end{array}$ & $\begin{array}{c}2.925^{* * *} \\
(1.11)\end{array}$ & $\begin{array}{c}5.373^{*} \\
(2.86)\end{array}$ & $\begin{array}{l}1.406 \\
(1.38)\end{array}$ & $\begin{array}{c}2.137^{* *} \\
(0.98)\end{array}$ \\
\hline Trade $(\varnothing \mathrm{t}-3$ to $\mathrm{t}-1)$ & $\begin{array}{r}-0.001 \\
(0.00)\end{array}$ & $\begin{array}{l}0.000 \\
(0.00)\end{array}$ & $\begin{array}{c}-0.000 \\
(0.00)\end{array}$ & $\begin{array}{r}-0.001 \\
(0.00)\end{array}$ & $\begin{array}{r}-0.001 \\
(0.00)\end{array}$ & $\begin{array}{l}-0.000 \\
(0.00)\end{array}$ \\
\hline Aid p.c., $\ln (\varnothing \mathrm{t}-3$ to $\mathrm{t}-1)$ & $\begin{array}{r}-0.012 \\
(0.02)\end{array}$ & $\begin{array}{l}-0.007 \\
(0.01)\end{array}$ & $\begin{array}{r}-0.002 \\
(0.01)\end{array}$ & $\begin{array}{l}-0.008 \\
(0.03)\end{array}$ & $\begin{array}{l}-0.006 \\
(0.01)\end{array}$ & $\begin{array}{r}-0.010 \\
(0.01)\end{array}$ \\
\hline Member of GATT/WTO, t-1 & $\begin{array}{l}-0.036 \\
(0.05)\end{array}$ & $\begin{array}{l}0.005 \\
(0.02)\end{array}$ & $\begin{array}{l}0.010 \\
(0.02)\end{array}$ & $\begin{array}{l}-0.012 \\
(0.05)\end{array}$ & $\begin{array}{r}-0.040 \\
(0.03)\end{array}$ & $\begin{array}{l}-0.007 \\
(0.03)\end{array}$ \\
\hline \# measures available, $\ln$ & $\begin{array}{l}0.031 \\
(0.38) \\
\end{array}$ & $\begin{array}{c}-0.983^{* * *} \\
(0.24) \\
\end{array}$ & $\begin{array}{c}0.742^{* *} \\
(0.32)\end{array}$ & $\begin{array}{l}0.272 \\
(0.59) \\
\end{array}$ & $\begin{array}{l}0.042 \\
(0.58) \\
\end{array}$ & $\begin{array}{l}0.019 \\
(0.28) \\
\end{array}$ \\
\hline $\begin{array}{l}\mathrm{N} \\
\text { Adj. R2 }\end{array}$ & $\begin{array}{c}572 \\
0.206\end{array}$ & $\begin{array}{l}1782 \\
0.087\end{array}$ & $\begin{array}{l}1805 \\
0.101\end{array}$ & $\begin{array}{c}681 \\
0.111\end{array}$ & $\begin{array}{l}2072 \\
0.084\end{array}$ & $\begin{array}{l}2096 \\
0.070\end{array}$ \\
\hline
\end{tabular}

b) core indicators-V-Dem ordinal

\begin{tabular}{|c|c|c|c|c|c|c|}
\hline & $(7)$ & $(8)$ & $(9)$ & $(10)$ & (11) & $(12)$ \\
\hline & Breakdown & Backsliding & $\begin{array}{c}\text { Breakdown \& } \\
\text { Backsliding }\end{array}$ & Transition & Liberalization & $\begin{array}{c}\text { Transition \& } \\
\text { Liberalization }\end{array}$ \\
\hline Successful coup in $t$ & $\begin{array}{c}0.096^{* * *} \\
(0.02)\end{array}$ & $\begin{array}{c}0.171^{* * *} \\
(0.02)\end{array}$ & $\begin{array}{c}0.143^{* * *} \\
(0.02)\end{array}$ & $\begin{array}{c}0.082^{*} \\
(0.05)\end{array}$ & $\begin{array}{l}0.027 \\
(0.02)\end{array}$ & $\begin{array}{c}0.054 * * * \\
(0.02)\end{array}$ \\
\hline Election in $\mathrm{t}$ & $\begin{array}{l}0.006 \\
(0.02)\end{array}$ & $\begin{array}{c}-0.002 \\
(0.01)\end{array}$ & $\begin{array}{c}-0.000 \\
(0.01)\end{array}$ & $\begin{array}{c}0.036 * * \\
(0.01)\end{array}$ & $\begin{array}{c}0.065^{* * *} \\
(0.01)\end{array}$ & $\begin{array}{c}0.054 * * * \\
(0.01)\end{array}$ \\
\hline Population, $\ln (\varnothing \mathrm{t}-3$ to $\mathrm{t}-1)$ & $\begin{array}{l}0.315 \\
(2.15)\end{array}$ & $\begin{array}{l}0.968 \\
(1.43)\end{array}$ & $\begin{array}{l}-1.381 \\
(1.01)\end{array}$ & $\begin{array}{c}-5.544^{*} \\
(2.81)\end{array}$ & $\begin{array}{l}-1.065 \\
(1.07)\end{array}$ & $\begin{array}{c}-2.052^{* *} \\
(0.90)\end{array}$ \\
\hline GDP p.c., $\ln (\varnothing \mathrm{t}-3$ to $\mathrm{t}-1)$ & $\begin{array}{l}0.009 \\
(2.17)\end{array}$ & $\begin{array}{l}0.815 \\
(1.45)\end{array}$ & $\begin{array}{l}-1.586 \\
(1.01)\end{array}$ & $\begin{array}{c}-5.374^{*} \\
(2.87)\end{array}$ & $\begin{array}{l}-1.211 \\
(1.10)\end{array}$ & $\begin{array}{c}-2.157 * * \\
(0.92)\end{array}$ \\
\hline GDP, $\ln (\varnothing \mathrm{t}-3$ to $\mathrm{t}-1)$ & $\begin{array}{r}-0.109 \\
(2.17)\end{array}$ & $\begin{array}{l}-0.807 \\
(1.44)\end{array}$ & $\begin{array}{l}1.570 \\
(1.01)\end{array}$ & $\begin{array}{c}5.373^{*} \\
(2.86)\end{array}$ & $\begin{array}{l}1.097 \\
(1.10)\end{array}$ & $\begin{array}{c}2.074^{* *} \\
(0.92)\end{array}$ \\
\hline Trade $(\varnothing \mathrm{t}-3$ to $\mathrm{t}-1)$ & $\begin{array}{l}-0.001 \\
(0.00)\end{array}$ & $\begin{array}{l}0.000 \\
(0.00)\end{array}$ & $\begin{array}{r}-0.000 \\
(0.00)\end{array}$ & $\begin{array}{r}-0.001 \\
(0.00)\end{array}$ & $\begin{array}{c}-0.001 * \\
(0.00)\end{array}$ & $\begin{array}{l}-0.000 \\
(0.00)\end{array}$ \\
\hline Aid p.c., $\ln (\varnothing \mathrm{t}-3$ to t-1) & $\begin{array}{l}-0.012 \\
(0.02)\end{array}$ & $\begin{array}{l}0.001 \\
(0.01)\end{array}$ & $\begin{array}{l}0.001 \\
(0.01)\end{array}$ & $\begin{array}{l}-0.008 \\
(0.03)\end{array}$ & $\begin{array}{l}-0.011 \\
(0.01)\end{array}$ & $\begin{array}{r}-0.011 \\
(0.01)\end{array}$ \\
\hline Member of GATT/WTO, t-1 & $\begin{array}{l}-0.036 \\
(0.05)\end{array}$ & $\begin{array}{c}0.043^{*} \\
(0.02)\end{array}$ & $\begin{array}{l}0.027 \\
(0.02)\end{array}$ & $\begin{array}{l}-0.012 \\
(0.05)\end{array}$ & $\begin{array}{l}-0.005 \\
(0.03)\end{array}$ & $\begin{array}{l}0.008 \\
(0.03)\end{array}$ \\
\hline \# measures available, $\ln$ & $\begin{array}{l}0.031 \\
(0.38) \\
\end{array}$ & $\begin{array}{c}-0.886^{* * *} \\
(0.25) \\
\end{array}$ & $\begin{array}{c}0.697^{* *} \\
(0.33)\end{array}$ & $\begin{array}{l}0.272 \\
(0.59) \\
\end{array}$ & $\begin{array}{c}-0.054 \\
(0.70) \\
\end{array}$ & $\begin{array}{l}0.020 \\
(0.28) \\
\end{array}$ \\
\hline $\begin{array}{l}\mathrm{N} \\
\text { Adj. R2 }\end{array}$ & $\begin{array}{c}572 \\
0.206 \\
\end{array}$ & $\begin{array}{c}1831 \\
0.098 \\
\end{array}$ & $\begin{array}{l}1852 \\
0.107 \\
\end{array}$ & $\begin{array}{c}681 \\
0.111 \\
\end{array}$ & $\begin{array}{l}2111 \\
0.075 \\
\end{array}$ & $\begin{array}{l}2141 \\
0.064 \\
\end{array}$ \\
\hline
\end{tabular}


c) extended set of indicators-V-Dem continuous

\begin{tabular}{|c|c|c|c|c|c|c|}
\hline & $\left(7^{\prime}\right)$ & $\left(8^{\prime}\right)$ & $\left(9^{\prime}\right)$ & $\left(10^{\prime}\right)$ & $\left(11^{\prime}\right)$ & $\left(12^{\prime}\right)$ \\
\hline & Breakdown & Backsliding & $\begin{array}{c}\text { Breakdown \& } \\
\text { Backsliding }\end{array}$ & Transition & Liberalization & $\begin{array}{c}\text { Transition \& } \\
\text { Liberalization }\end{array}$ \\
\hline Successful coup in $t$ & $\begin{array}{c}0.085^{* * *} \\
(0.02)\end{array}$ & $\begin{array}{c}0.174 * * * \\
(0.02)\end{array}$ & $\begin{array}{c}0.138^{* * *} \\
(0.02)\end{array}$ & $\begin{array}{c}0.064 * * \\
(0.03)\end{array}$ & $\begin{array}{l}0.043 \\
(0.03)\end{array}$ & $\begin{array}{l}0.009 \\
(0.01)\end{array}$ \\
\hline Election in $\mathrm{t}$ & $\begin{array}{c}-0.002 \\
(0.01)\end{array}$ & $\begin{array}{l}0.011 \\
(0.01)\end{array}$ & $\begin{array}{l}0.008 \\
(0.01)\end{array}$ & $\begin{array}{c}0.044^{* * *} \\
(0.01)\end{array}$ & $\begin{array}{c}0.061 * * * \\
(0.01)\end{array}$ & $\begin{array}{c}0.103^{* * *} \\
(0.01)\end{array}$ \\
\hline Population, $\ln (\varnothing \mathrm{t}-3$ to $\mathrm{t}-1)$ & $\begin{array}{l}1.302 \\
(1.60)\end{array}$ & $\begin{array}{l}1.014 \\
(1.36)\end{array}$ & $\begin{array}{c}-0.590 \\
(0.82)\end{array}$ & $\begin{array}{c}-1.612^{* *} \\
(0.76)\end{array}$ & $\begin{array}{c}-2.026 \\
(1.28)\end{array}$ & $\begin{array}{c}-0.552 \\
(0.38)\end{array}$ \\
\hline GDP p.c., $\ln (\varnothing \mathrm{t}-3$ to t- 1$)$ & $\begin{array}{l}0.989 \\
(1.61)\end{array}$ & $\begin{array}{l}0.875 \\
(1.38)\end{array}$ & $\begin{array}{l}-0.745 \\
(0.84)\end{array}$ & $\begin{array}{c}-1.577^{*} \\
(0.82)\end{array}$ & $\begin{array}{c}-2.138^{*} \\
(1.29)\end{array}$ & $\begin{array}{c}-0.593 \\
(0.39)\end{array}$ \\
\hline GDP, $\ln (\varnothing \mathrm{t}-3$ to $\mathrm{t}-1)$ & $\begin{array}{c}-0.988 \\
(1.61)\end{array}$ & $\begin{array}{l}-0.875 \\
(1.38)\end{array}$ & $\begin{array}{l}0.731 \\
(0.84)\end{array}$ & $\begin{array}{c}1.520 * \\
(0.81)\end{array}$ & $\begin{array}{l}2.021 \\
(1.29)\end{array}$ & $\begin{array}{l}0.555 \\
(0.39)\end{array}$ \\
\hline Trade $(\varnothing \mathrm{t}-3$ to $\mathrm{t}-1)$ & $\begin{array}{r}-0.001 \\
(0.00)\end{array}$ & $\begin{array}{r}-0.000 \\
(0.00)\end{array}$ & $\begin{array}{r}-0.000 \\
(0.00)\end{array}$ & $\begin{array}{r}-0.000 \\
(0.00)\end{array}$ & $\begin{array}{l}0.000 \\
(0.00)\end{array}$ & $\begin{array}{c}-0.000 \\
(0.00)\end{array}$ \\
\hline Aid p.c., $\ln (\varnothing \mathrm{t}-3$ to t-1) & $\begin{array}{l}0.002 \\
(0.01)\end{array}$ & $\begin{array}{r}-0.001 \\
(0.01)\end{array}$ & $\begin{array}{r}-0.001 \\
(0.01)\end{array}$ & $\begin{array}{c}-0.014 \\
(0.01)\end{array}$ & $\begin{array}{c}-0.020 * * \\
(0.01)\end{array}$ & $\begin{array}{c}-0.009 * * \\
(0.00)\end{array}$ \\
\hline Member of GATT/WTO, t-1 & $\begin{array}{c}-0.023 \\
(0.03)\end{array}$ & $\begin{array}{l}0.035 \\
(0.02)\end{array}$ & $\begin{array}{l}0.021 \\
(0.02)\end{array}$ & $\begin{array}{l}0.022 \\
(0.04)\end{array}$ & $\begin{array}{c}-0.002 \\
(0.03)\end{array}$ & $\begin{array}{l}0.004 \\
(0.01)\end{array}$ \\
\hline \# measures available, $\ln$ & $\begin{array}{c}1.004 * * * \\
(0.36)\end{array}$ & $\begin{array}{c}-0.581 * \\
(0.31)\end{array}$ & $\begin{array}{l}0.030 \\
(0.17) \\
\end{array}$ & $\begin{array}{c}-0.043 \\
(0.16) \\
\end{array}$ & $\begin{array}{c}-0.252 \\
(0.37) \\
\end{array}$ & $\begin{array}{l}0.006 \\
(0.09) \\
\end{array}$ \\
\hline $\begin{array}{l}\mathrm{N} \\
\text { Adj. R2 }\end{array}$ & $\begin{array}{c}966 \\
0.162\end{array}$ & $\begin{array}{l}2032 \\
0.077\end{array}$ & $\begin{array}{l}2067 \\
0.083\end{array}$ & $\begin{array}{l}1145 \\
0.084\end{array}$ & $\begin{array}{l}2401 \\
0.070\end{array}$ & $\begin{array}{l}2423 \\
0.158\end{array}$ \\
\hline
\end{tabular}

d) extended set of indicators-V-Dem ordinal

\begin{tabular}{|c|c|c|c|c|c|c|}
\hline & $\left(7^{\prime}\right)$ & $\left(8^{\prime}\right)$ & $\left(9^{\prime}\right)$ & $\left(10^{\prime}\right)$ & $\left(11^{\prime}\right)$ & $\left(12^{\prime}\right)$ \\
\hline & Breakdown & Backsliding & $\begin{array}{c}\text { Breakdown \& } \\
\text { Backsliding } \\
\end{array}$ & Transition & Liberalization & $\begin{array}{c}\text { Transition \& } \\
\text { Liberalization } \\
\end{array}$ \\
\hline Successful coup in $t$ & $\begin{array}{c}0.085^{* * *} \\
(0.02)\end{array}$ & $\begin{array}{c}0.157 * * * \\
(0.02)\end{array}$ & $\begin{array}{c}0.131^{* * *} \\
(0.02)\end{array}$ & $\begin{array}{c}0.064^{* *} \\
(0.03)\end{array}$ & $\begin{array}{c}0.044^{*} \\
(0.02)\end{array}$ & $\begin{array}{l}0.008 \\
(0.01)\end{array}$ \\
\hline Election in $\mathrm{t}$ & $\begin{array}{c}-0.002 \\
(0.01)\end{array}$ & $\begin{array}{l}0.007 \\
(0.01)\end{array}$ & $\begin{array}{l}0.006 \\
(0.01)\end{array}$ & $\begin{array}{c}0.044 * * * \\
(0.01)\end{array}$ & $\begin{array}{c}0.063^{* * *} \\
(0.01)\end{array}$ & $\begin{array}{c}0.096 * * * \\
(0.01)\end{array}$ \\
\hline Population, $\ln (\varnothing \mathrm{t}-3$ to t- 1$)$ & $\begin{array}{l}1.302 \\
(1.60)\end{array}$ & $\begin{array}{l}1.066 \\
(1.46)\end{array}$ & $\begin{array}{l}0.039 \\
(1.08)\end{array}$ & $\begin{array}{c}-1.612^{* *} \\
(0.76)\end{array}$ & $\begin{array}{c}-1.548 \\
(1.24)\end{array}$ & $\begin{array}{c}-0.423 \\
(0.34)\end{array}$ \\
\hline GDP p.c., $\ln (\varnothing \mathrm{t}-3$ to $\mathrm{t}-1)$ & $\begin{array}{l}0.989 \\
(1.61)\end{array}$ & $\begin{array}{l}0.922 \\
(1.47)\end{array}$ & $\begin{array}{l}-0.109 \\
(1.09)\end{array}$ & $\begin{array}{c}-1.577^{*} \\
(0.82)\end{array}$ & $\begin{array}{l}-1.711 \\
(1.25)\end{array}$ & $\begin{array}{l}-0.461 \\
(0.34)\end{array}$ \\
\hline GDP, $\ln (\varnothing \mathrm{t}-3$ to $\mathrm{t}-1)$ & $\begin{array}{r}-0.988 \\
(1.61)\end{array}$ & $\begin{array}{l}-0.925 \\
(1.47)\end{array}$ & $\begin{array}{l}0.092 \\
(1.09)\end{array}$ & $\begin{array}{c}1.520^{*} \\
(0.81)\end{array}$ & $\begin{array}{l}1.588 \\
(1.26)\end{array}$ & $\begin{array}{l}0.424 \\
(0.34)\end{array}$ \\
\hline Trade $(\varnothing \mathrm{t}-3$ to $\mathrm{t}-1)$ & $\begin{array}{r}-0.001 \\
(0.00)\end{array}$ & $\begin{array}{l}0.000 \\
(0.00)\end{array}$ & $\begin{array}{r}-0.000 \\
(0.00)\end{array}$ & $\begin{array}{r}-0.000 \\
(0.00)\end{array}$ & $\begin{array}{l}0.000 \\
(0.00)\end{array}$ & $\begin{array}{r}-0.000 \\
(0.00)\end{array}$ \\
\hline Aid p.c., $\ln (\varnothing \mathrm{t}-3$ to t-1) & $\begin{array}{l}0.002 \\
(0.01)\end{array}$ & $\begin{array}{l}-0.009 \\
(0.01)\end{array}$ & $\begin{array}{l}-0.006 \\
(0.01)\end{array}$ & $\begin{array}{l}-0.014 \\
(0.01)\end{array}$ & $\begin{array}{c}-0.024 * * * \\
(0.01)\end{array}$ & $\begin{array}{c}-0.010^{* * *} \\
(0.00)\end{array}$ \\
\hline Member of GATT/WTO, t-1 & $\begin{array}{c}-0.023 \\
(0.03)\end{array}$ & $\begin{array}{l}0.033 \\
(0.02)\end{array}$ & $\begin{array}{l}0.018 \\
(0.02)\end{array}$ & $\begin{array}{l}0.022 \\
(0.04)\end{array}$ & $\begin{array}{l}0.003 \\
(0.03)\end{array}$ & $\begin{array}{l}0.006 \\
(0.01)\end{array}$ \\
\hline \# measures available, $\ln$ & $\begin{array}{c}1.004^{* * *} \\
(0.36)\end{array}$ & $\begin{array}{c}-0.506^{*} \\
(0.27)\end{array}$ & $\begin{array}{l}0.059 \\
(0.16) \\
\end{array}$ & $\begin{array}{c}-0.043 \\
(0.16) \\
\end{array}$ & $\begin{array}{r}-0.236 \\
(0.34) \\
\end{array}$ & $\begin{array}{r}-0.020 \\
(0.07) \\
\end{array}$ \\
\hline $\begin{array}{l}\mathrm{N} \\
\text { Adj. R2 }\end{array}$ & $\begin{array}{c}966 \\
0.162\end{array}$ & $\begin{array}{l}2100 \\
0.066\end{array}$ & $\begin{array}{l}2130 \\
0.075\end{array}$ & $\begin{array}{l}1145 \\
0.084\end{array}$ & $\begin{array}{l}2443 \\
0.073\end{array}$ & $\begin{array}{l}2467 \\
0.158\end{array}$ \\
\hline
\end{tabular}




\section{Appendix III: Results of the Robustness Checks-Extended Set of Indicators}

\section{a) Rupture}

\begin{tabular}{|c|c|c|c|c|c|c|c|c|c|}
\hline & \multicolumn{5}{|c|}{ TRANSITION } & \multicolumn{4}{|c|}{ BREAKDOWN } \\
\hline \multirow[t]{2}{*}{ Indicator name } & scope & \multicolumn{2}{|c|}{ Pevehouse 2002b } & \multicolumn{2}{|c|}{ Donno 2013} & \multirow[t]{2}{*}{ scope } & \multicolumn{2}{|c|}{ Andersen et al. 2014} & \multirow{2}{*}{$\begin{array}{c}\text { Houle } 2009 \\
T 4, M 1\end{array}$} \\
\hline & & $T 2, M 1$ & $T 2, M 3$ & $T 1, M 3$ & $T 1, M 5$ & & $T 1, M 3$ & $T 1, M 6$ & \\
\hline Bernhard et al. 2001 & AUT only & NA & NA & $\mathrm{NA}$ & NA & DEM only & & & \\
\hline Boix et al. 2013 & AUT only & & & & & DEM only & & & \\
\hline Cheibub et al. 2010 & AUT only & & & & & DEM only & & & \\
\hline Freedom House (FIW: free) & AUT only & & & & & DEM only & & & \\
\hline Freedom House (FIW: partly free) & AUT only & & & & & DEM only & & & \\
\hline Freedom House (PR: free) & AUT only & & & & & DEM only & & & \\
\hline Freedom House (PR: partly free) & AUT only & & & & & DEM only & & & \\
\hline Freedom House (FIW: bybrid) & AUT only & & & & & DEM only & & & \\
\hline Freedom House (PR: bybrid) & AUT only & & & & & DEM only & & & \\
\hline Gasiorowski 1996 & AUT only & & & & & DEM only & & & \\
\hline Gasiorowski 1996 (bybrid) & AUT only & & & & & DEM only & & & \\
\hline Gates 2006 & AUT only & & & & & DEM only & & & \\
\hline Geddes et al. 2014 & AUT only & & & & & DEM only & & & \\
\hline Magaloni et al. 2013 & AUT only & & & & & DEM only & & & \\
\hline Polity IV $(A N=A U T)$ & AUT only & & & & & DEM only & & & \\
\hline Polity IV $(A N=D E M)$ & AUT only & & & & & DEM only & & & \\
\hline Polity IV (threshold: 7) & AUT only & & & & & DEM only & & & \\
\hline Polity IV (threshold: 1) & AUT only & & & & & DEM only & & & \\
\hline Polity IV (exrec \& parcomp) & AUT only & & & & & DEM only & & & \\
\hline Polity IV (bybrid: 6 \& -6) & AUT only & & & & & DEM only & & & \\
\hline Polity IV (bybrid: $8 \& 1$ ) & AUT only & & & & & DEM only & & & \\
\hline Przeworski et al. 2000 & AUT only & & & & & DEM only & & & \\
\hline Reich 2002 & AUT only & & & & & DEM only & & & \\
\hline Reich 2002 (bybrid) & AUT only & & & & & DEM only & & & \\
\hline Svolik 2012 & AUT only & & & & & DEM only & & & \\
\hline Ulfelder 2012 & AUT only & & & & & DEM only & & & \\
\hline Wabman et al. 2013 & AUT only & & & & & DEM only & & & \\
\hline & $\%$ success & $61.5 \%$ & $46.2 \%$ & $\begin{array}{l}0.0 \% \\
61.5 \%\end{array}$ & $\begin{array}{l}0.0 \% \\
42.3 \%\end{array}$ & $\%$ success & $55.6 \%$ & $18.5 \%$ & $25.9 \%$ \\
\hline
\end{tabular}


b) Reform

\begin{tabular}{|c|c|c|c|c|c|c|c|c|c|c|c|}
\hline \multirow{3}{*}{ Coding DV } & \multicolumn{6}{|c|}{ LIBERALIZATION } & \multicolumn{5}{|c|}{ BACKSLIDING } \\
\hline & \multirow{2}{*}{ scope } & \multicolumn{2}{|c|}{$\begin{array}{l}\text { Burke and Leigh } \\
2010\end{array}$} & \multirow{2}{*}{$\begin{array}{c}\begin{array}{c}\text { Cornell } \\
2013\end{array} \\
T 1, M 3 \\
\end{array}$} & \multicolumn{2}{|c|}{$\begin{array}{c}\text { Ansell and } \\
\text { Samuels } 2010\end{array}$} & \multirow{2}{*}{ scope } & \multicolumn{2}{|c|}{$\begin{array}{c}\text { Burke and Leigh } \\
2010\end{array}$} & \multirow{2}{*}{$\begin{array}{c}\text { Gibler and } \\
\text { Randazzo } \\
2011 \\
T 2\end{array}$} & \multirow{2}{*}{$\begin{array}{r}\begin{array}{r}\text { Goldstone } \\
\text { et al. } 2010\end{array} \\
T 1, M 3\end{array}$} \\
\hline & & $T 3, M$ & $T 3, M 2$ & & $T 3, M C$ & $T 3, M E$ & & $T 3, M 3$ & $T 3, M 4$ & & \\
\hline Freedom House (FIW, any change) & all & & & & & & all & & & & \\
\hline Freedom House (PR, any change) & all & & & & & & all & & & & \\
\hline Freedom House (FIW, 1+ points) & all & & & & & & all & & & & \\
\hline Freedom House (PR, 1+ points) & all & & & & & & all & & & & \\
\hline Gasiorowski (bybrid) & all & & & & & & all & & & & \\
\hline Gates et al. 2006 (any change) & all & & & & & & all & & & & \\
\hline Polity IV (any change) & all & & & & & & all & & & & \\
\hline Polity IV (3+ points) & all & & & & & & all & & & & \\
\hline Polity IV (5+ points) & all & & & & & & all & & & & \\
\hline Reich 2002 (bybrid) & all & & & & & & all & & & & \\
\hline Skaaning et al. 2015 & all & & & & & & all & & & & \\
\hline Wabman et al. 2013 (any change) & all & & & & & & all & & & & \\
\hline Wabman et al. 2013 (1.5+ points) & all & & & & & & all & & & & \\
\hline Freedom House (FIW, any change) & AUT only & & & & & & AUT only & & & & \\
\hline Freedom House (PR, any change) & AUT only & & & & & & AUT only & & & & \\
\hline Freedom House (FIW, 1+ points) & AUT only & & & & & & AUT only & & & & \\
\hline Freedom House (PR, 1+ points) & AUT only & & & & & & AUT only & & & & \\
\hline Gasiorowski (bybrid) & AUT only & & & & & & AUT only & & & & \\
\hline Gates et al. 2006 (any change) & AUT only & & & & & & AUT only & & & & \\
\hline Polity IV (any change) & AUT only & & & & & & AUT only & & & & \\
\hline Polity IV (3+ points) & AUT only & & & & & & AUT only & & & & \\
\hline Polity IV (5+ points) & AUT only & & & & & & AUT only & & & & \\
\hline Reich 2002 (bybrid) & AUT only & & & & & & AUT only & & & & \\
\hline Skaaning et al. 2015 & AUT only & NA & $\mathrm{NA}$ & NA & $\mathrm{NA}$ & NA & AUT only & $\mathrm{NA}$ & NA & NA & NA \\
\hline Wabman et al. 2013 (any change) & AUT only & & & & & & AUT only & & & & \\
\hline Wabman et al. 2013 (1.5+ points) & AUT only & & & & & & AUT only & & & & \\
\hline & $\%$ success & $80 \%$ & $72 \%$ & $36 \%$ & $0 \%$ & $4 \%$ & $\%$ success & $36 \%$ & $36 \%$ & $56 \%$ & $8 \%$ \\
\hline & & & & $60 \%$ & $16 \%$ & $20 \%$ & & & & & $40 \%$ \\
\hline
\end{tabular}


Appendix IV: Robustness Checks: Pevehouse 2002

\begin{tabular}{|c|c|c|}
\hline \multicolumn{3}{|c|}{ Boix et al. 2013} \\
\hline & Mode1 1 & Model 3 \\
\hline \multirow[t]{2}{*}{ IOScore $_{i t-1}$} & $0.058^{* *}$ & 0.033 \\
\hline & $(0.03)$ & $(0.04)$ \\
\hline \multirow[t]{2}{*}{$\Delta$ IOScore $_{\text {it }-1}$} & 0.044 & 0.023 \\
\hline & $(0.06)$ & $(0.16)$ \\
\hline \multirow[t]{2}{*}{ pcGDP $_{\text {it-1 }}$} & -0.000 & -0.000 \\
\hline & $(0.00)$ & $(0.00)$ \\
\hline \multirow[t]{2}{*}{$\Delta \mathrm{pcGDP}$ it-1 } & -0.000 & -0.000 \\
\hline & $(0.00)$ & $(0.00)$ \\
\hline \multirow[t]{2}{*}{ Contagion $_{\mathrm{it}}$} & 0.023 & 0.025 \\
\hline & $(0.04)$ & $(0.04)$ \\
\hline \multirow[t]{2}{*}{ PastDem $_{\text {it }}$} & $1.579 * * *$ & $1.705^{* * *}$ \\
\hline & $(0.45)$ & $(0.44)$ \\
\hline \multirow[t]{2}{*}{ RegConflict $_{i t}$} & $-0.316^{*}$ & $-0.328^{*}$ \\
\hline & $(0.19)$ & $(0.18)$ \\
\hline \multirow[t]{2}{*}{ IntViolence $_{\mathrm{it}-1}$} & $2.109 * * *$ & $2.109 * * *$ \\
\hline & $(0.47)$ & $(0.47)$ \\
\hline \multirow[t]{2}{*}{ MilReg $_{\text {it }}$} & $-2.214^{* * *}$ & $-2.293 * * *$ \\
\hline & $(0.68)$ & $(0.71)$ \\
\hline \multirow[t]{2}{*}{ Indep $_{\text {it }}$} & $0.011 * *$ & $0.012 * * *$ \\
\hline & $(0.00)$ & $(0.00)$ \\
\hline \multirow[t]{2}{*}{ Constant } & $-6.293^{* * *}$ & $-6.273^{* * *}$ \\
\hline & $(0.61)$ & $(0.68)$ \\
\hline $\mathrm{N}$ & 2554 & 2555 \\
\hline Log Likelihood & -196.384 & -198.709 \\
\hline $\mathrm{X}^{2}$ & 60.960 & 56.982 \\
\hline Prob $>X^{2}$ & 0.000 & 0.000 \\
\hline Pseudo-R ${ }^{2}$ & 0.189 & 0.180 \\
\hline \multicolumn{3}{|c|}{$\begin{array}{l}*{ }^{*} \mathrm{p}<0.10, * * \mathrm{p}<0.05, * * * \mathrm{p}<0.01 . \text { Model } 3: \\
\text { including non-regional organizations and IFIs }\end{array}$} \\
\hline
\end{tabular}

\begin{tabular}{|c|c|c|}
\hline \multicolumn{3}{|c|}{ Cheibub et al. 2010} \\
\hline & Mode1 1 & Model 3 \\
\hline \multirow[t]{2}{*}{ IOScore $_{i t-1}$} & $0.071 * * *$ & 0.040 \\
\hline & $(0.03)$ & $(0.04)$ \\
\hline \multirow[t]{2}{*}{$\Delta$ IOScore $_{\text {it }-1}$} & 0.032 & -0.023 \\
\hline & $(0.07)$ & $(0.20)$ \\
\hline \multirow[t]{2}{*}{$\mathrm{pcGDP}_{\mathrm{it}-1}$} & -0.000 & -0.000 \\
\hline & $(0.00)$ & $(0.00)$ \\
\hline \multirow[t]{2}{*}{$\Delta \mathrm{pcGDP}_{\mathrm{it}-1}$} & -0.000 & -0.000 \\
\hline & $(0.00)$ & $(0.00)$ \\
\hline \multirow[t]{2}{*}{ Contagion $_{\text {it }}$} & 0.024 & 0.026 \\
\hline & $(0.04)$ & $(0.04)$ \\
\hline \multirow[t]{2}{*}{ PastDem $_{\text {it }}$} & $1.409 * * *$ & $1.545^{* * *}$ \\
\hline & $(0.45)$ & $(0.45)$ \\
\hline \multirow[t]{2}{*}{ RegConflict $_{i t}$} & $-0.324 *$ & $-0.332 * *$ \\
\hline & $(0.17)$ & $(0.17)$ \\
\hline \multirow[t]{2}{*}{ IntViolence $_{\mathrm{it}-1}$} & $2.184 * * *$ & $2.174 * * *$ \\
\hline & $(0.51)$ & $(0.50)$ \\
\hline \multirow[t]{2}{*}{ MilReg $_{i t}$} & $-2.244 * * *$ & $-2.326 * * *$ \\
\hline & $(0.76)$ & $(0.79)$ \\
\hline \multirow[t]{2}{*}{ Indepit } & $0.011^{* *}$ & $0.012^{* * *}$ \\
\hline & $(0.00)$ & $(0.00)$ \\
\hline \multirow[t]{2}{*}{ Constant } & $-6.365^{* * *}$ & $-6.326 * * *$ \\
\hline & $(0.60)$ & $(0.70)$ \\
\hline $\mathrm{N}$ & 2541 & 2542 \\
\hline Log Likelihood & -200.496 & -203.797 \\
\hline $\mathrm{X}^{2}$ & 71.620 & 60.991 \\
\hline Prob $>X^{2}$ & 0.000 & 0.000 \\
\hline Pseudo-R ${ }^{2}$ & 0.197 & 0.184 \\
\hline
\end{tabular}

\begin{tabular}{|c|c|c|}
\hline \multicolumn{3}{|c|}{ FH: free, FIW } \\
\hline & Model 1 & Model 3 \\
\hline \multirow[t]{2}{*}{ IOScore $_{i t-1}$} & -0.019 & -0.074 \\
\hline & $(0.04)$ & $(0.05)$ \\
\hline \multirow[t]{2}{*}{$\Delta$ IOScore $_{\mathrm{it}-1}$} & $0.172^{* *}$ & -0.068 \\
\hline & $(0.08)$ & $(0.42)$ \\
\hline \multirow[t]{2}{*}{ pcGDP $P_{i t-1}$} & -0.000 & -0.000 \\
\hline & $(0.00)$ & $(0.00)$ \\
\hline \multirow[t]{2}{*}{$\Delta \mathrm{pcGDP}_{\mathrm{it}-1}$} & -0.000 & -0.000 \\
\hline & $(0.00)$ & $(0.00)$ \\
\hline \multirow[t]{2}{*}{ Contagion $_{\text {it }}$} & 0.049 & 0.049 \\
\hline & $(0.04)$ & $(0.04)$ \\
\hline \multirow[t]{2}{*}{ PastDem $_{\text {it }}$} & $1.815^{* * *}$ & $1.832^{* * *}$ \\
\hline & $(0.68)$ & $(0.68)$ \\
\hline \multirow[t]{2}{*}{ RegConflict $_{i t}$} & -0.453 & -0.454 \\
\hline & $(0.36)$ & $(0.35)$ \\
\hline \multirow[t]{2}{*}{ IntViolence $_{\mathrm{it}-1}$} & $2.248^{* * *}$ & $2.103^{* * *}$ \\
\hline & $(0.78)$ & $(0.68)$ \\
\hline \multirow[t]{2}{*}{ MilReg $_{i t}$} & 0.000 & 0.000 \\
\hline & (.) & (.) \\
\hline \multirow[t]{2}{*}{ Indep $_{i t}$} & $0.010^{*}$ & $0.012 * *$ \\
\hline & $(0.01)$ & $(0.01)$ \\
\hline \multirow[t]{2}{*}{ Constant } & $-5.883 * * *$ & $-5.054 * * *$ \\
\hline & $(0.81)$ & $(0.86)$ \\
\hline $\mathrm{N}$ & 1120 & 1120 \\
\hline Log Likelihood & -91.830 & -92.291 \\
\hline $\mathrm{X}^{2}$ & 37.959 & 41.048 \\
\hline Prob $>X^{2}$ & 0.000 & 0.000 \\
\hline Pseudo-R ${ }^{2}$ & 0.181 & 0.177 \\
\hline
\end{tabular}




\begin{tabular}{|c|c|c|}
\hline \multicolumn{3}{|c|}{ FH: partly free, FIW } \\
\hline & Model 1 & Model 3 \\
\hline IOScore $_{\mathrm{it}-1}$ & $\begin{array}{c}0.066^{* *} \\
(0.03)\end{array}$ & $\begin{array}{c}0.120 * * * \\
(0.05)\end{array}$ \\
\hline$\Delta$ IOScore $_{\mathrm{it}-1}$ & $\begin{array}{c}-0.030 \\
(0.06)\end{array}$ & $\begin{array}{c}-0.044 \\
(0.11)\end{array}$ \\
\hline pcGDP $P_{\mathrm{it}-1}$ & $\begin{array}{c}0.000^{* *} \\
(0.00)\end{array}$ & $\begin{array}{c}0.000^{* *} \\
(0.00)\end{array}$ \\
\hline$\Delta \mathrm{pcGDP} \mathrm{it}-1$ & $\begin{array}{c}-0.000 \\
(0.00)\end{array}$ & $\begin{array}{c}-0.000 \\
(0.00)\end{array}$ \\
\hline Contagion $_{\text {it }}$ & $\begin{array}{l}-0.010 \\
(0.02)\end{array}$ & $\begin{array}{l}-0.003 \\
(0.02)\end{array}$ \\
\hline PastDem $_{\text {it }}$ & $\begin{array}{c}0.778^{*} \\
(0.47)\end{array}$ & $\begin{array}{l}0.749 \\
(0.50)\end{array}$ \\
\hline RegConflict $_{\mathrm{it}}$ & $\begin{array}{r}-0.167 \\
(0.20)\end{array}$ & $\begin{array}{c}-0.163 \\
(0.21)\end{array}$ \\
\hline IntViolence $_{\text {it- } 1}$ & $\begin{array}{c}0.888^{* * *} \\
(0.30)\end{array}$ & $\begin{array}{c}0.911 * * * \\
(0.30)\end{array}$ \\
\hline MilRegit $_{\text {it }}$ & $\begin{array}{c}-0.284 \\
(0.36)\end{array}$ & $\begin{array}{c}-0.259 \\
(0.37)\end{array}$ \\
\hline Indep $_{\text {it }}$ & $\begin{array}{l}0.001 \\
(0.00)\end{array}$ & $\begin{array}{l}0.000 \\
(0.00)\end{array}$ \\
\hline Constant & $\begin{array}{c}-3.468^{* * *} \\
(0.37)\end{array}$ & $\begin{array}{c}-4.419 * * * \\
(0.59)\end{array}$ \\
\hline $\mathrm{N}$ & 867 & 867 \\
\hline Log Likelihood & -237.705 & -235.826 \\
\hline $\mathrm{X}^{2}$ & 28.047 & 32.269 \\
\hline Prob $>X^{2}$ & 0.002 & 0.000 \\
\hline Pseudo- $\mathrm{R}^{2}$ & 0.069 & 0.076 \\
\hline
\end{tabular}

\begin{tabular}{|c|c|c|}
\hline \multicolumn{3}{|c|}{ FH: free, PR } \\
\hline & Model 1 & Mode1 3 \\
\hline IOScore $_{i t-1}$ & $\begin{array}{l}0.002 \\
(0.04)\end{array}$ & $\begin{array}{c}-0.054 \\
(0.05)\end{array}$ \\
\hline$\Delta$ IOScore $_{\mathrm{it}-1}$ & $\begin{array}{c}0.152^{*} \\
(0.08)\end{array}$ & $\begin{array}{c}-0.106 \\
(0.42)\end{array}$ \\
\hline $\mathrm{pcGDP}_{\mathrm{it}-1}$ & $\begin{array}{r}-0.000 \\
(0.00)\end{array}$ & $\begin{array}{r}-0.000 \\
(0.00)\end{array}$ \\
\hline$\Delta \mathrm{pcGDP}_{\mathrm{it}-1}$ & $\begin{array}{r}-0.000 \\
(0.00)\end{array}$ & $\begin{array}{r}-0.000 \\
(0.00)\end{array}$ \\
\hline Contagion $_{\text {it }}$ & $\begin{array}{l}0.033 \\
(0.05)\end{array}$ & $\begin{array}{l}0.037 \\
(0.04)\end{array}$ \\
\hline PastDem $_{\text {it }}$ & $\begin{array}{c}1.680^{* * *} \\
(0.62)\end{array}$ & $\begin{array}{c}1.707 * * * \\
(0.62)\end{array}$ \\
\hline RegConflict $_{\text {it }}$ & $\begin{array}{c}-0.446 \\
(0.35)\end{array}$ & $\begin{array}{c}-0.445 \\
(0.34)\end{array}$ \\
\hline IntViolence $_{\text {it }-1}$ & $\begin{array}{c}2.165^{* * *} \\
(0.77)\end{array}$ & $\begin{array}{c}2.047^{* * *} \\
(0.70)\end{array}$ \\
\hline MilReg $_{\text {it }}$ & $\begin{array}{c}0.000 \\
(.)\end{array}$ & $\begin{array}{c}0.000 \\
(.)\end{array}$ \\
\hline Indep $_{\text {it }}$ & $\begin{array}{c}0.010^{*} \\
(0.01)\end{array}$ & $\begin{array}{c}0.013^{* *} \\
(0.01)\end{array}$ \\
\hline Constant & $\begin{array}{c}-5.886^{* * *} \\
(0.79)\end{array}$ & $\begin{array}{c}-5.186^{* * *} \\
(0.87)\end{array}$ \\
\hline $\mathrm{N}$ & 1121 & 1121 \\
\hline Log Likelihood & -92.547 & -93.089 \\
\hline $\mathrm{X}^{2}$ & 34.300 & 37.633 \\
\hline Prob $>X^{2}$ & 0.000 & 0.000 \\
\hline Pseudo-R ${ }^{2}$ & 0.175 & 0.170 \\
\hline
\end{tabular}

\begin{tabular}{|c|c|c|}
\hline \multicolumn{3}{|c|}{ FH: partly free, PR } \\
\hline & Model 1 & Mode1 3 \\
\hline IOScore $_{i t-1}$ & $\begin{array}{c}0.086^{* * *} \\
(0.03)\end{array}$ & $\begin{array}{c}0.146^{* * *} \\
(0.04)\end{array}$ \\
\hline$\Delta$ IOScore $_{\mathrm{it}-1}$ & $\begin{array}{r}-0.027 \\
(0.06)\end{array}$ & $\begin{array}{c}-0.219 \\
(0.24)\end{array}$ \\
\hline pcGDP $P_{i t-1}$ & $\begin{array}{c}0.000^{* *} \\
(0.00)\end{array}$ & $\begin{array}{c}0.000^{* *} \\
(0.00)\end{array}$ \\
\hline$\Delta \mathrm{pcGDP}_{\mathrm{it}-1}$ & $\begin{array}{l}-0.000 \\
(0.00)\end{array}$ & $\begin{array}{c}-0.000 \\
(0.00)\end{array}$ \\
\hline Contagion $_{\text {it }}$ & $\begin{array}{l}-0.025 \\
(0.02)\end{array}$ & $\begin{array}{l}-0.016 \\
(0.02)\end{array}$ \\
\hline PastDemit & $\begin{array}{l}0.542 \\
(0.49)\end{array}$ & $\begin{array}{l}0.514 \\
(0.50)\end{array}$ \\
\hline RegConflict $_{\text {it }}$ & $\begin{array}{c}-0.148 \\
(0.20)\end{array}$ & $\begin{array}{c}-0.146 \\
(0.21)\end{array}$ \\
\hline IntViolence $_{\mathrm{it}-1}$ & $\begin{array}{c}0.806^{* * * *} \\
(0.25)\end{array}$ & $\begin{array}{c}0.821 * * * \\
(0.25)\end{array}$ \\
\hline MilReg $_{i t}$ & $\begin{array}{c}-0.462 \\
(0.33)\end{array}$ & $\begin{array}{c}-0.410 \\
(0.34)\end{array}$ \\
\hline Indep ${ }_{i t}$ & $\begin{array}{l}0.000 \\
(0.00)\end{array}$ & $\begin{array}{l}0.001 \\
(0.00)\end{array}$ \\
\hline Constant & $\begin{array}{c}-3.552 * * * \\
(0.34)\end{array}$ & $\begin{array}{c}-4.685^{* * *} \\
(0.53)\end{array}$ \\
\hline $\mathrm{N}$ & 998 & 998 \\
\hline Log Likelihood & -246.239 & -244.027 \\
\hline $\mathrm{X}^{2}$ & 33.894 & 38.791 \\
\hline Prob $>X^{2}$ & 0.000 & 0.000 \\
\hline Pseudo-R ${ }^{2}$ & 0.066 & 0.075 \\
\hline $\begin{array}{l}* \mathrm{p}<0.10, * * \\
\text { including non-1 }\end{array}$ & $\begin{array}{l}5, * * * \mathrm{p}<0 . \\
\text { al organiza }\end{array}$ & $\begin{array}{l}\text { Model 3: } \\
\text { is and IFIs }\end{array}$ \\
\hline
\end{tabular}


FH: HYB, FIW

convergence

not achieved

\begin{tabular}{|c|c|c|}
\hline \multicolumn{3}{|c|}{ FH: HYB, PR } \\
\hline & Model 1 & Model 3 \\
\hline IOScore $_{\mathrm{it}-1}$ & $\begin{array}{c}-0.221 * * * \\
(0.07)\end{array}$ & $\begin{array}{c}-0.272^{* * *} \\
(0.05)\end{array}$ \\
\hline$\Delta$ IOScore $_{\text {it }-1}$ & $\begin{array}{l}0.341 \\
(0.23)\end{array}$ & $\begin{array}{c}-0.411 \\
(0.37)\end{array}$ \\
\hline pcGDP $_{\mathrm{it}-1}$ & $\begin{array}{c}-0.000 \\
(0.00)\end{array}$ & $\begin{array}{c}-0.000 \\
(0.00)\end{array}$ \\
\hline$\Delta \mathrm{pcGDP} \mathrm{P}_{\mathrm{it}-1}$ & $\begin{array}{l}0.000 \\
(0.00)\end{array}$ & $\begin{array}{l}0.001 \\
(0.00)\end{array}$ \\
\hline Contagion $_{\text {it }}$ & $\begin{array}{c}0.157^{*} \\
(0.09)\end{array}$ & $\begin{array}{l}0.104 \\
(0.10)\end{array}$ \\
\hline PastDem $_{\text {it }}$ & $\begin{array}{c}0.000 \\
(.)\end{array}$ & $\begin{array}{c}0.000 \\
(.)\end{array}$ \\
\hline RegConflict $_{\text {it }}$ & $\begin{array}{c}-0.942 * \\
(0.55)\end{array}$ & $\begin{array}{c}-1.005^{*} \\
(0.53)\end{array}$ \\
\hline IntViolence $_{\mathrm{it}-1}$ & $\begin{array}{c}0.000 \\
(.)\end{array}$ & $\begin{array}{c}0.000 \\
(.)\end{array}$ \\
\hline MilRegit $_{\text {it }}$ & $\begin{array}{c}0.000 \\
(.)\end{array}$ & $\begin{array}{c}0.000 \\
(.)\end{array}$ \\
\hline Indepit & $\begin{array}{l}0.007 \\
(0.01)\end{array}$ & $\begin{array}{l}0.011 \\
(0.01)\end{array}$ \\
\hline Constant & $\begin{array}{c}-3.423^{* * *} \\
(1.00)\end{array}$ & $\begin{array}{c}-1.706^{* *} \\
(0.72)\end{array}$ \\
\hline $\mathrm{N}$ & 446 & 446 \\
\hline Log Likelihood & -22.472 & -21.022 \\
\hline $\mathrm{X}^{2}$ & 55.149 & 87.105 \\
\hline Prob $>X^{2}$ & 0.000 & 0.000 \\
\hline Pseudo-R ${ }^{2}$ & 0.181 & 0.234 \\
\hline
\end{tabular}

\begin{tabular}{|c|c|c|}
\hline \multicolumn{3}{|c|}{ Gasiorowski 1996} \\
\hline & Model 1 & Mode1 3 \\
\hline \multirow[t]{2}{*}{ IOScore $_{i t-1}$} & 0.054 & $0.108^{* *}$ \\
\hline & $(0.04)$ & $(0.05)$ \\
\hline \multirow[t]{2}{*}{$\Delta$ IOScore $_{\text {it }-1}$} & 0.009 & -0.278 \\
\hline & $(0.05)$ & $(0.28)$ \\
\hline \multirow[t]{2}{*}{ pcGDP $P_{i t-1}$} & -0.000 & -0.000 \\
\hline & $(0.00)$ & $(0.00)$ \\
\hline \multirow[t]{2}{*}{$\Delta \mathrm{pcGDP}_{\mathrm{it}-1}$} & 0.000 & 0.000 \\
\hline & $(0.00)$ & $(0.00)$ \\
\hline \multirow[t]{2}{*}{ Contagion $_{\text {it }}$} & $0.218^{* * *}$ & $0.223^{* * *}$ \\
\hline & $(0.07)$ & $(0.07)$ \\
\hline \multirow[t]{2}{*}{ PastDem it $_{\text {it }}$} & $1.227 * * *$ & $1.279 * * *$ \\
\hline & $(0.38)$ & $(0.40)$ \\
\hline \multirow[t]{2}{*}{ RegConflict $_{\text {it }}$} & -0.247 & -0.257 \\
\hline & $(0.17)$ & $(0.17)$ \\
\hline \multirow[t]{2}{*}{ IntViolence $_{\text {it-1 }}$} & $1.056^{* * *}$ & $1.031 * * *$ \\
\hline & $(0.39)$ & $(0.39)$ \\
\hline \multirow[t]{2}{*}{ MilReg $_{i t}$} & $-1.338 * * *$ & $-1.317 * * *$ \\
\hline & $(0.45)$ & $(0.46)$ \\
\hline \multirow[t]{2}{*}{ Indepit } & 0.002 & 0.002 \\
\hline & $(0.01)$ & $(0.00)$ \\
\hline \multirow[t]{2}{*}{ Constant } & $-5.351 * * *$ & $-6.255^{* * *}$ \\
\hline & $(0.46)$ & $(0.70)$ \\
\hline $\mathrm{N}$ & 1957 & 1958 \\
\hline Log Likelihood & -202.393 & -200.460 \\
\hline $\mathrm{X}^{2}$ & 63.143 & 55.670 \\
\hline Prob $>X^{2}$ & 0.000 & 0.000 \\
\hline Pseudo-R ${ }^{2}$ & 0.116 & 0.125 \\
\hline \multicolumn{3}{|c|}{$\begin{array}{l}* \mathrm{p}<0.10, * * \mathrm{p}<0.05, * * * \mathrm{p}<0.01 . \text { Model } 3 \text { : } \\
\text { including non-regional organizations and IFIs }\end{array}$} \\
\hline
\end{tabular}




\begin{tabular}{|c|c|c|}
\hline \multicolumn{3}{|c|}{ Gasiorowski 1996: HYB } \\
\hline & Model 1 & Mode1 3 \\
\hline IOScore $_{i t-1}$ & $\begin{array}{c}-0.002 \\
(0.06)\end{array}$ & $\begin{array}{l}0.022 \\
(0.07)\end{array}$ \\
\hline$\Delta$ IOScore $_{\mathrm{it}-1}$ & $\begin{array}{c}0.111^{* *} \\
(0.05)\end{array}$ & $\begin{array}{c}-0.473^{*} \\
(0.29)\end{array}$ \\
\hline pcGDP $_{\text {it }-1}$ & $\begin{array}{c}-0.000 \\
(0.00)\end{array}$ & $\begin{array}{c}-0.000 \\
(0.00)\end{array}$ \\
\hline$\Delta \mathrm{pcGDP}_{\mathrm{it}-1}$ & $\begin{array}{c}-0.000 \\
(0.00)\end{array}$ & $\begin{array}{l}0.000 \\
(0.00)\end{array}$ \\
\hline Contagion $_{\text {it }}$ & $\begin{array}{c}0.243^{* * *} \\
(0.09)\end{array}$ & $\begin{array}{c}0.258^{* * *} \\
(0.10)\end{array}$ \\
\hline PastDemit & $\begin{array}{c}2.460^{* * *} \\
(0.55)\end{array}$ & $\begin{array}{c}2.534 * * * \\
(0.51)\end{array}$ \\
\hline RegConflict ${ }_{i t}$ & $\begin{array}{r}-0.267 \\
(0.28)\end{array}$ & $\begin{array}{c}-0.240 \\
(0.29)\end{array}$ \\
\hline IntViolence $_{\mathrm{it}-1}$ & $\begin{array}{l}0.747 \\
(0.59)\end{array}$ & $\begin{array}{l}0.608 \\
(0.62)\end{array}$ \\
\hline MilReg $_{\text {it }}$ & $\begin{array}{c}-1.999 * * * \\
(0.55)\end{array}$ & $\begin{array}{c}-1.961^{* * *} \\
(0.52)\end{array}$ \\
\hline Indep $_{\text {it }}$ & $\begin{array}{l}0.004 \\
(0.01)\end{array}$ & $\begin{array}{l}0.004 \\
(0.01)\end{array}$ \\
\hline Constant & $\begin{array}{c}-6.150 * * * \\
(0.62)\end{array}$ & $\begin{array}{c}-6.440^{* * *} \\
(0.89)\end{array}$ \\
\hline $\mathrm{N}$ & 2159 & 2160 \\
\hline Log Likelihood & -95.583 & -94.582 \\
\hline $\mathrm{X}^{2}$ & 49.489 & 50.160 \\
\hline Prob $>X^{2}$ & 0.000 & 0.000 \\
\hline Pseudo- $\mathrm{R}^{2}$ & 0.158 & 0.167 \\
\hline
\end{tabular}

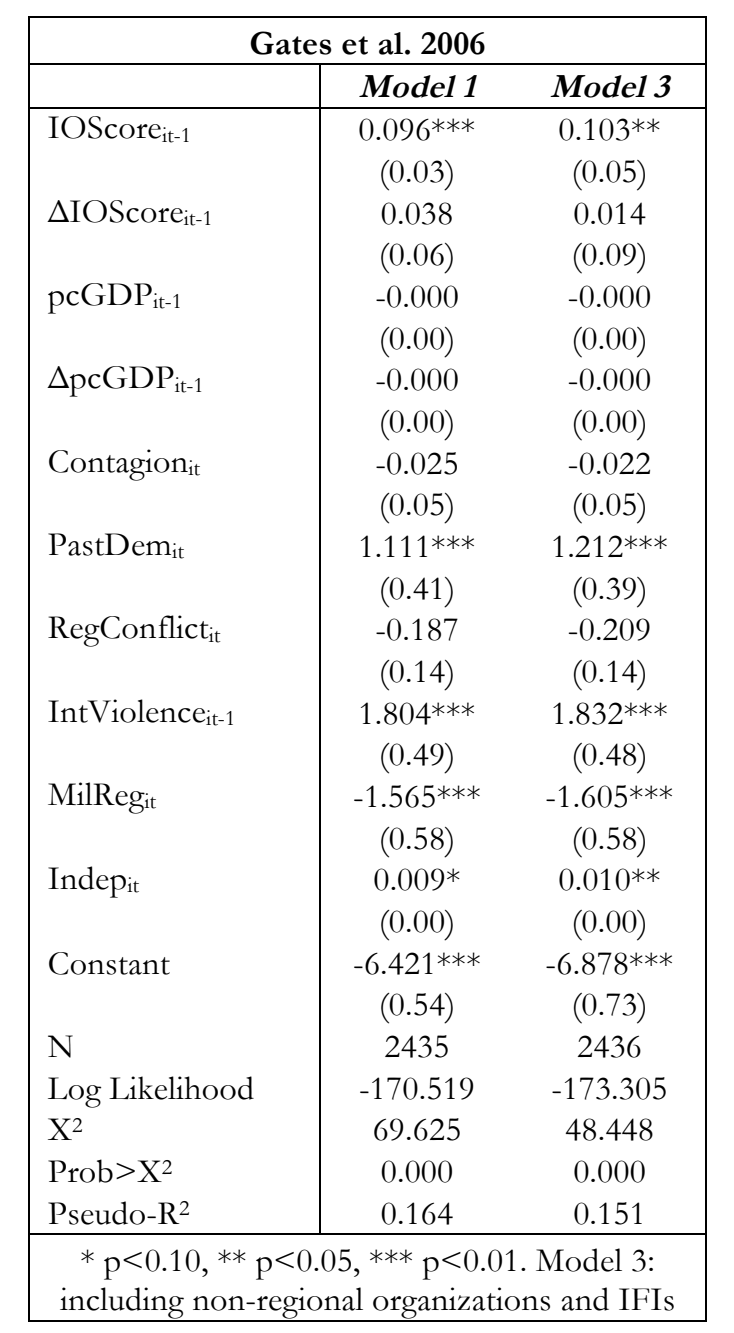

\begin{tabular}{|c|c|c|}
\hline \multicolumn{3}{|c|}{ Geddes et al. 2014} \\
\hline & Model 1 & Model 3 \\
\hline IOScore $_{i t-1}$ & $\begin{array}{c}0.078^{* * *} \\
(0.03)\end{array}$ & $\begin{array}{c}0.069^{*} \\
(0.04)\end{array}$ \\
\hline$\Delta$ IOScore $_{\text {it }-1}$ & $\begin{array}{l}0.073 \\
(0.05)\end{array}$ & $\begin{array}{l}0.017 \\
(0.18)\end{array}$ \\
\hline pcGDP $P_{i t-1}$ & $\begin{array}{r}-0.000 \\
(0.00)\end{array}$ & $\begin{array}{c}-0.000 \\
(0.00)\end{array}$ \\
\hline$\Delta p c G D P_{i t-1}$ & $\begin{array}{c}-0.000 \\
(0.00)\end{array}$ & $\begin{array}{c}-0.000 \\
(0.00)\end{array}$ \\
\hline Contagion $_{\text {it }}$ & $\begin{array}{l}0.040 \\
(0.04)\end{array}$ & $\begin{array}{l}0.043 \\
(0.04)\end{array}$ \\
\hline PastDemit $_{\text {it }}$ & $\begin{array}{c}1.641^{* * *} \\
(0.48)\end{array}$ & $\begin{array}{c}1.762^{* * *} \\
(0.48)\end{array}$ \\
\hline RegConflict $_{\text {it }}$ & $\begin{array}{c}-0.399 * * \\
(0.19)\end{array}$ & $\begin{array}{c}-0.399 * * \\
(0.18)\end{array}$ \\
\hline IntViolence $_{\mathrm{it}-1}$ & $\begin{array}{c}1.959^{* * *} \\
(0.50)\end{array}$ & $\begin{array}{c}1.946 * * * \\
(0.49)\end{array}$ \\
\hline MilRegit $_{i t}$ & $\begin{array}{c}-1.732^{* * *} \\
(0.61)\end{array}$ & $\begin{array}{c}-1.819^{* * *} \\
(0.62)\end{array}$ \\
\hline Indepit & $\begin{array}{l}0.007 \\
(0.01)\end{array}$ & $\begin{array}{c}0.00)^{*} \\
(0.00)\end{array}$ \\
\hline Constant & $\begin{array}{c}-6.478^{* * *} \\
(0.62)\end{array}$ & $\begin{array}{c}-6.696^{* * *} \\
(0.78)\end{array}$ \\
\hline $\mathrm{N}$ & 2544 & 2544 \\
\hline Log Likelihood & -180.224 & -183.228 \\
\hline $\mathrm{X}^{2}$ & 64.090 & 40.547 \\
\hline Prob $>X^{2}$ & 0.000 & 0.000 \\
\hline Pseudo-R ${ }^{2}$ & 0.174 & 0.160 \\
\hline
\end{tabular}




\begin{tabular}{|c|c|c|}
\hline \multicolumn{3}{|c|}{ Magaloni et al. 2013} \\
\hline & Model 1 & Model 3 \\
\hline IOScore $_{i t-1}$ & $\begin{array}{c}0.098^{* * *} \\
(0.03)\end{array}$ & $\begin{array}{c}0.100^{* * *} \\
(0.04)\end{array}$ \\
\hline$\Delta$ IOScore $_{\text {it }-1}$ & $\begin{array}{l}0.011 \\
(0.05)\end{array}$ & $\begin{array}{r}-0.227 \\
(0.27)\end{array}$ \\
\hline pcGDP $P_{\text {it }-1}$ & $\begin{array}{l}-0.000 \\
(0.00)\end{array}$ & $\begin{array}{l}-0.000 \\
(0.00)\end{array}$ \\
\hline$\Delta \mathrm{pcGDP}_{\mathrm{it}-1}$ & $\begin{array}{r}-0.000 \\
(0.00)\end{array}$ & $\begin{array}{r}-0.000 \\
(0.00)\end{array}$ \\
\hline Contagion $_{\text {it }}$ & $\begin{array}{l}0.043 \\
(0.04)\end{array}$ & $\begin{array}{l}0.049 \\
(0.04)\end{array}$ \\
\hline PastDemit & $\begin{array}{c}1.845^{* * *} \\
(0.40)\end{array}$ & $\begin{array}{c}2.020^{* * *} \\
(0.40)\end{array}$ \\
\hline RegConflict ${ }_{i t}$ & $\begin{array}{c}-0.431^{* *} \\
(0.18)\end{array}$ & $\begin{array}{c}-0.430^{* *} \\
(0.17)\end{array}$ \\
\hline IntViolence $_{\mathrm{it}-1}$ & $\begin{array}{c}2.025^{* * *} \\
(0.53)\end{array}$ & $\begin{array}{c}1.992^{* * *} \\
(0.51)\end{array}$ \\
\hline MilReg $_{\text {it }}$ & $\begin{array}{c}-2.383^{* * *} \\
(0.80)\end{array}$ & $\begin{array}{c}-2.474^{* * *} \\
(0.82)\end{array}$ \\
\hline Indep $_{\text {it }}$ & $\begin{array}{c}0.008^{*} \\
(0.00)\end{array}$ & $\begin{array}{c}0.009 * * \\
(0.00)\end{array}$ \\
\hline Constant & $\begin{array}{c}-6.827 * * * \\
(0.56)\end{array}$ & $\begin{array}{c}-7.240^{* * *} \\
(0.64)\end{array}$ \\
\hline $\mathrm{N}$ & 2646 & 2646 \\
\hline Log Likelihood & -181.012 & -183.333 \\
\hline $\mathrm{X}^{2}$ & 81.225 & 67.147 \\
\hline Prob $>X^{2}$ & 0.000 & 0.000 \\
\hline Pseudo-R ${ }^{2}$ & 0.220 & 0.210 \\
\hline
\end{tabular}

\begin{tabular}{|c|c|c|}
\hline \multicolumn{3}{|c|}{ Polity IV: AN=AUT } \\
\hline & Model 1 & Mode1 3 \\
\hline IOScore $_{i t-1}$ & $\begin{array}{c}0.089^{* * *} \\
(0.03)\end{array}$ & $\begin{array}{c}0.102^{* * *} \\
(0.04)\end{array}$ \\
\hline$\Delta \mathrm{IOS}_{\text {core }} \mathrm{it}_{\mathrm{t}-1}$ & $\begin{array}{l}0.093 \\
(0.06)\end{array}$ & $\begin{array}{r}-0.320 \\
(0.36)\end{array}$ \\
\hline pcGDP $P_{i t-1}$ & $\begin{array}{r}-0.000 \\
(0.00)\end{array}$ & $\begin{array}{r}-0.000 \\
(0.00)\end{array}$ \\
\hline$\Delta \mathrm{pcGDP}_{\mathrm{it}-1}$ & $\begin{array}{c}-0.000 \\
(0.00)\end{array}$ & $\begin{array}{c}-0.000 \\
(0.00)\end{array}$ \\
\hline Contagion $_{\text {it }}$ & $\begin{array}{c}0.058^{*} \\
(0.03)\end{array}$ & $\begin{array}{c}0.068^{* *} \\
(0.03)\end{array}$ \\
\hline PastDem it & $\begin{array}{c}1.821^{* * *} \\
(0.33)\end{array}$ & $\begin{array}{c}1.937 * * * \\
(0.33)\end{array}$ \\
\hline RegConflict ${ }_{i t}$ & $\begin{array}{c}-0.476^{* * * *} \\
(0.17)\end{array}$ & $\begin{array}{c}-0.475^{* * *} \\
(0.17)\end{array}$ \\
\hline IntViolence $_{\text {it- } 1}$ & $\begin{array}{c}2.334 * * * \\
(0.60)\end{array}$ & $\begin{array}{c}2.260 * * * \\
(0.57)\end{array}$ \\
\hline MilReg $_{\text {it }}$ & $\begin{array}{c}-1.591 * * * \\
(0.57)\end{array}$ & $\begin{array}{c}-1.611^{* * *} \\
(0.58)\end{array}$ \\
\hline Indepit & $\begin{array}{l}0.004 \\
(0.00)\end{array}$ & $\begin{array}{l}0.005 \\
(0.00)\end{array}$ \\
\hline Constant & $\begin{array}{c}-7.075^{* * *} \\
(0.57)\end{array}$ & $\begin{array}{c}-7.541^{* * *} \\
(0.66)\end{array}$ \\
\hline $\mathrm{N}$ & 2736 & 2737 \\
\hline Log Likelihood & -188.289 & -190.298 \\
\hline $\mathrm{X}^{2}$ & 108.376 & 105.408 \\
\hline Prob $>X^{2}$ & 0.000 & 0.000 \\
\hline Pseudo-R ${ }^{2}$ & 0.208 & 0.199 \\
\hline $\begin{array}{l}{ }^{*} \mathrm{p}<0.10,{ }^{*} \\
\text { including non-1 }\end{array}$ & $\begin{array}{l}5, * * * \mathrm{p}<0 . \\
\text { al organizat }\end{array}$ & $\begin{array}{l}\text { Model 3: } \\
\text { is and IFIs }\end{array}$ \\
\hline
\end{tabular}

\begin{tabular}{|c|c|c|}
\hline \multicolumn{3}{|c|}{ Polity IV: AN=DEM } \\
\hline & Model 1 & Mode1 3 \\
\hline IOScore $_{\text {it }-1}$ & $\begin{array}{c}0.093^{* * *} \\
(0.02)\end{array}$ & $\begin{array}{c}0.133^{* * *} \\
(0.04)\end{array}$ \\
\hline$\Delta$ IOScore $_{\mathrm{it}-1}$ & $\begin{array}{c}-0.093 \\
(0.06)\end{array}$ & $\begin{array}{r}-0.117 \\
(0.12)\end{array}$ \\
\hline pcGDP $P_{i t-1}$ & $\begin{array}{l}-0.000 \\
(0.00)\end{array}$ & $\begin{array}{l}-0.000 \\
(0.00)\end{array}$ \\
\hline$\Delta \mathrm{pcGDP}_{\mathrm{it}-1}$ & $\begin{array}{c}-0.000 \\
(0.00)\end{array}$ & $\begin{array}{c}-0.000 \\
(0.00)\end{array}$ \\
\hline Contagion $_{\text {it }}$ & $\begin{array}{l}0.039 \\
(0.03)\end{array}$ & $\begin{array}{l}0.043 \\
(0.03)\end{array}$ \\
\hline PastDem ${ }_{\text {it }}$ & $\begin{array}{c}1.147^{* *} \\
(0.48)\end{array}$ & $\begin{array}{c}1.175^{* *} \\
(0.49)\end{array}$ \\
\hline RegConflict $_{\text {it }}$ & $\begin{array}{c}-0.521 * * * \\
(0.19)\end{array}$ & $\begin{array}{c}-0.524^{* * * *} \\
(0.18)\end{array}$ \\
\hline IntViolence $_{i t-1}$ & $\begin{array}{c}1.093^{* * *} \\
(0.38)\end{array}$ & $\begin{array}{c}1.108^{* * *} \\
(0.38)\end{array}$ \\
\hline MilReg $_{i t}$ & $\begin{array}{c}-0.090 \\
(0.40)\end{array}$ & $\begin{array}{r}-0.057 \\
(0.40)\end{array}$ \\
\hline Indepit & $\begin{array}{l}0.000 \\
(0.00)\end{array}$ & $\begin{array}{l}0.001 \\
(0.00)\end{array}$ \\
\hline Constant & $\begin{array}{c}-5.084 * * * \\
(0.38)\end{array}$ & $\begin{array}{c}-6.011 * * * \\
(0.54)\end{array}$ \\
\hline $\mathrm{N}$ & 2009 & 2009 \\
\hline Log Likelihood & -215.090 & -215.367 \\
\hline $\mathrm{X}^{2}$ & 63.576 & 58.590 \\
\hline Prob $>X^{2}$ & 0.000 & 0.000 \\
\hline Pseudo-R ${ }^{2}$ & 0.095 & 0.094 \\
\hline $\begin{array}{l}* \mathrm{p}<0.10,{ }^{* *} \\
\text { including non-1 }\end{array}$ & $\begin{array}{l}5, * * * \mathrm{p}<0 . \\
\text { al organizat }\end{array}$ & $\begin{array}{l}\text { Model 3: } \\
\text { Is and IFIs }\end{array}$ \\
\hline
\end{tabular}




\begin{tabular}{|c|c|c|}
\hline \multicolumn{3}{|c|}{ Polity IV: threshold 7} \\
\hline & Model 1 & Mode1 3 \\
\hline IOScore $_{i t-1}$ & $\begin{array}{c}0.123^{* * *} \\
(0.04)\end{array}$ & $\begin{array}{c}0.133^{* *} \\
(0.06)\end{array}$ \\
\hline$\Delta$ IOScore $_{\mathrm{it}-1}$ & $\begin{array}{l}0.109 \\
(0.08)\end{array}$ & $\begin{array}{c}-0.098 \\
(0.35)\end{array}$ \\
\hline pcGDP $P_{i t-1}$ & $\begin{array}{c}-0.000 \\
(0.00)\end{array}$ & $\begin{array}{c}-0.000 \\
(0.00)\end{array}$ \\
\hline$\Delta \mathrm{pcGDP}_{\mathrm{it}-1}$ & $\begin{array}{c}-0.000 \\
(0.00)\end{array}$ & $\begin{array}{c}-0.000 \\
(0.00)\end{array}$ \\
\hline Contagion $_{\text {it }}$ & $\begin{array}{c}0.064^{*} \\
(0.04)\end{array}$ & $\begin{array}{c}0.074^{*} \\
(0.04)\end{array}$ \\
\hline PastDem $_{\text {it }}$ & $\begin{array}{c}2.013^{* * *} \\
(0.47)\end{array}$ & $\begin{array}{c}2.087 * * * \\
(0.47)\end{array}$ \\
\hline RegConflict $_{\text {it }}$ & $\begin{array}{c}-0.450^{* *} \\
(0.19)\end{array}$ & $\begin{array}{c}-0.433^{* *} \\
(0.18)\end{array}$ \\
\hline IntViolence $_{\mathrm{it}-1}$ & $\begin{array}{c}2.623 * * * \\
(0.84)\end{array}$ & $\begin{array}{c}2.536 * * * \\
(0.79)\end{array}$ \\
\hline MilReg $_{i t}$ & $\begin{array}{c}-1.465^{* *} \\
(0.64)\end{array}$ & $\begin{array}{c}-1.524^{* *} \\
(0.64)\end{array}$ \\
\hline Indepit & $\begin{array}{l}0.004 \\
(0.00)\end{array}$ & $\begin{array}{l}0.005 \\
(0.00)\end{array}$ \\
\hline Constant & $\begin{array}{c}-8.359 * * * \\
(0.91)\end{array}$ & $\begin{array}{c}-8.813^{* * *} \\
(1.06)\end{array}$ \\
\hline $\mathrm{N}$ & 2741 & 2742 \\
\hline Log Likelihood & -134.691 & -138.712 \\
\hline $\mathrm{X}^{2}$ & 90.251 & 88.664 \\
\hline Prob $>X^{2}$ & 0.000 & 0.000 \\
\hline Pseudo-R ${ }^{2}$ & 0.246 & 0.224 \\
\hline
\end{tabular}

\begin{tabular}{|c|c|c|}
\hline \multicolumn{3}{|c|}{ Polity IV: threshold 1} \\
\hline & Model 1 & Mode1 3 \\
\hline IOScore $_{i t-1}$ & $\begin{array}{c}0.098^{* * *} \\
(0.03)\end{array}$ & $\begin{array}{c}0.129 * * * \\
(0.04)\end{array}$ \\
\hline$\Delta$ IOScore $_{\mathrm{it}-1}$ & $\begin{array}{l}0.013 \\
(0.06)\end{array}$ & $\begin{array}{c}-0.497 * * \\
(0.19)\end{array}$ \\
\hline pcGDP $P_{i t-1}$ & $\begin{array}{r}-0.000 \\
(0.00)\end{array}$ & $\begin{array}{c}-0.000 \\
(0.00)\end{array}$ \\
\hline$\Delta \mathrm{pcGDP}_{\mathrm{it}-1}$ & $\begin{array}{r}-0.000 \\
(0.00)\end{array}$ & $\begin{array}{r}-0.000 \\
(0.00)\end{array}$ \\
\hline Contagion $_{i t}$ & $\begin{array}{l}0.035 \\
(0.04)\end{array}$ & $\begin{array}{l}0.042 \\
(0.04)\end{array}$ \\
\hline PastDem $_{\text {it }}$ & $\begin{array}{c}1.533^{* * *} \\
(0.42)\end{array}$ & $\begin{array}{c}1.684 * * * \\
(0.43)\end{array}$ \\
\hline RegConflict ${ }_{i t}$ & $\begin{array}{c}-0.374 \\
(0.24)\end{array}$ & $\begin{array}{c}-0.404^{*} \\
(0.23)\end{array}$ \\
\hline IntViolence $_{\text {it- } 1}$ & $\begin{array}{c}1.607^{* * * *} \\
(0.40)\end{array}$ & $\begin{array}{c}1.580^{* * *} \\
(0.39)\end{array}$ \\
\hline MilReg $_{i t}$ & $\begin{array}{c}-1.486^{* * * *} \\
(0.50)\end{array}$ & $\begin{array}{c}-1.505^{* * *} \\
(0.52)\end{array}$ \\
\hline Indepit & $\begin{array}{l}0.004 \\
(0.00)\end{array}$ & $\begin{array}{l}0.005 \\
(0.00)\end{array}$ \\
\hline Constant & $\begin{array}{c}-5.725^{* * *} \\
(0.48)\end{array}$ & $\begin{array}{c}-6.529 * * * \\
(0.63)\end{array}$ \\
\hline $\mathrm{N}$ & 2434 & 2435 \\
\hline Log Likelihood & -209.384 & -207.978 \\
\hline $\mathrm{X}^{2}$ & 64.059 & 65.049 \\
\hline Prob $>X^{2}$ & 0.000 & 0.000 \\
\hline Pseudo-R ${ }^{2}$ & 0.154 & 0.160 \\
\hline $\begin{array}{l}* \mathrm{p}<0.10,{ }^{*} \\
\text { including non-1 }\end{array}$ & $\begin{array}{l}5, * * * \mathrm{p}<0 . \\
\text { al organiza }\end{array}$ & $\begin{array}{l}\text { Model 3: } \\
\text { as and IFIs }\end{array}$ \\
\hline
\end{tabular}

\begin{tabular}{|c|c|c|}
\hline \multicolumn{3}{|c|}{ Polity IV: exrec \& parcomp } \\
\hline & Model 1 & Mode1 3 \\
\hline IOScore $_{\mathrm{it}-1}$ & $\begin{array}{c}0.110^{* * *} \\
(0.03)\end{array}$ & $\begin{array}{c}0.114^{* *} \\
(0.05)\end{array}$ \\
\hline$\Delta$ IOScore $_{\mathrm{it}-1}$ & $\begin{array}{l}0.012 \\
(0.07)\end{array}$ & $\begin{array}{c}-0.055 \\
(0.11)\end{array}$ \\
\hline pcGDP $P_{\text {it }-1}$ & $\begin{array}{l}-0.000 \\
(0.00)\end{array}$ & $\begin{array}{c}-0.000 \\
(0.00)\end{array}$ \\
\hline$\Delta \mathrm{pcGDP}_{\mathrm{it}-1}$ & $\begin{array}{c}-0.000 \\
(0.00)\end{array}$ & $\begin{array}{c}-0.001 \\
(0.00)\end{array}$ \\
\hline Contagion $_{\text {it }}$ & $\begin{array}{l}0.009 \\
(0.04)\end{array}$ & $\begin{array}{l}0.015 \\
(0.04)\end{array}$ \\
\hline PastDemit & $\begin{array}{c}1.991^{* * *} \\
(0.52)\end{array}$ & $\begin{array}{c}2.111^{* * *} \\
(0.52)\end{array}$ \\
\hline RegConflict ${ }_{i t}$ & $\begin{array}{c}-0.418 \\
(0.26)\end{array}$ & $\begin{array}{c}-0.411^{*} \\
(0.24)\end{array}$ \\
\hline IntViolence $_{i t-1}$ & $\begin{array}{c}1.491 * * * \\
(0.48)\end{array}$ & $\begin{array}{c}1.471 * * * \\
(0.47)\end{array}$ \\
\hline MilReg $_{i t}$ & $\begin{array}{c}-1.673^{* *} \\
(0.72)\end{array}$ & $\begin{array}{c}-1.758^{* *} \\
(0.73)\end{array}$ \\
\hline Indepit & $\begin{array}{c}0.008^{*} \\
(0.00)\end{array}$ & $\begin{array}{c}0.010^{* *} \\
(0.00)\end{array}$ \\
\hline Constant & $\begin{array}{c}-6.618^{* * *} \\
(0.53)\end{array}$ & $\begin{array}{c}-7.093^{* * *} \\
(0.71)\end{array}$ \\
\hline $\mathrm{N}$ & 2469 & 2470 \\
\hline Log Likelihood & -147.979 & -150.824 \\
\hline $\mathrm{X}^{2}$ & 79.549 & 60.819 \\
\hline Prob $>X^{2}$ & 0.000 & 0.000 \\
\hline Pseudo-R ${ }^{2}$ & 0.195 & 0.179 \\
\hline
\end{tabular}




\begin{tabular}{|c|c|c|}
\hline \multicolumn{3}{|c|}{ Polity IV: HYB; thresholds: 6 \& -6} \\
\hline & Model 1 & Model 3 \\
\hline \multirow[t]{2}{*}{ IOScore $_{i t-1}$} & 0.062 & 0.050 \\
\hline & $(0.08)$ & $(0.12)$ \\
\hline \multirow[t]{2}{*}{$\Delta$ IOScore $_{\mathrm{it}-1}$} & -0.020 & 0.031 \\
\hline & $(0.10)$ & $(0.08)$ \\
\hline \multirow[t]{2}{*}{ pcGDP $P_{i t-1}$} & 0.000 & 0.000 \\
\hline & $(0.00)$ & $(0.00)$ \\
\hline \multirow[t]{2}{*}{$\Delta \mathrm{pcGDP}_{\mathrm{it}-1}$} & -0.000 & -0.000 \\
\hline & $(0.00)$ & $(0.00)$ \\
\hline \multirow[t]{2}{*}{ Contagion $_{\text {it }}$} & $0.157 * *$ & $0.165^{* *}$ \\
\hline & $(0.06)$ & $(0.06)$ \\
\hline \multirow[t]{2}{*}{ PastDem $_{\text {it }}$} & $2.214 *$ & $2.312 *$ \\
\hline & $(1.23)$ & $(1.21)$ \\
\hline \multirow[t]{2}{*}{ RegConflict ${ }_{i t}$} & -0.182 & -0.185 \\
\hline & $(0.25)$ & $(0.24)$ \\
\hline \multirow[t]{2}{*}{ IntViolence $_{\mathrm{it}-1}$} & $1.984 *$ & $2.014 *$ \\
\hline & $(1.09)$ & $(1.10)$ \\
\hline \multirow[t]{2}{*}{ MilReg $_{i t}$} & -1.165 & -1.239 \\
\hline & $(1.86)$ & $(1.88)$ \\
\hline \multirow[t]{2}{*}{ Indep $_{\text {it }}$} & -0.001 & 0.000 \\
\hline & $(0.01)$ & $(0.01)$ \\
\hline Constant & $\begin{array}{c}-9.145^{* * *} \\
(1.09)\end{array}$ & $\begin{array}{c}-9.311 * * * \\
(1.07)\end{array}$ \\
\hline $\mathrm{N}$ & 2736 & 2737 \\
\hline Log Likelihood & -47.999 & -48.333 \\
\hline $\mathrm{X}^{2}$ & 65.358 & 40.925 \\
\hline Prob $>X^{2}$ & 0.000 & 0.000 \\
\hline Pseudo-R ${ }^{2}$ & 0.206 & 0.200 \\
\hline
\end{tabular}

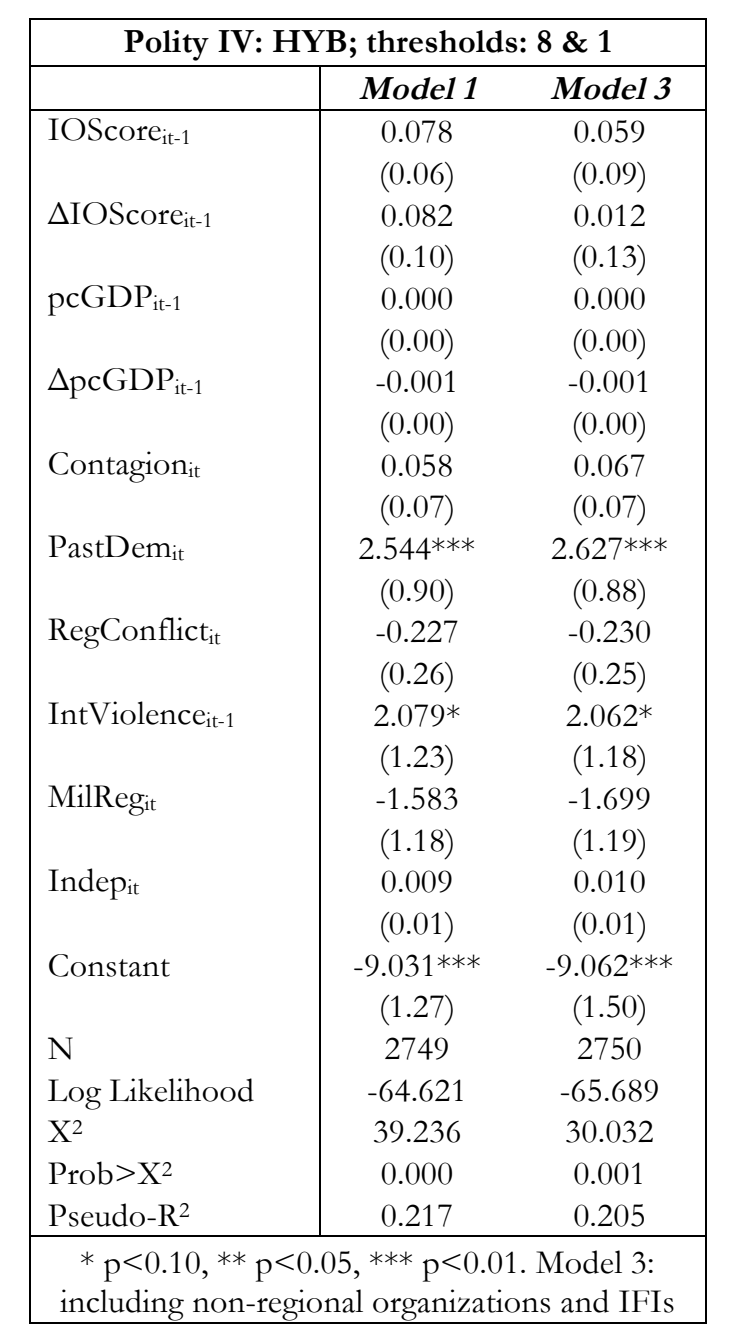

\begin{tabular}{|c|c|c|}
\hline \multicolumn{3}{|c|}{ Przeworski et al. 2000} \\
\hline & Model 1 & Mode1 3 \\
\hline IOScore $_{i t-1}$ & $\begin{array}{c}0.074 * * * \\
(0.03)\end{array}$ & $\begin{array}{l}0.049 \\
(0.04)\end{array}$ \\
\hline$\Delta$ IOScore $_{\mathrm{it}-1}$ & $\begin{array}{l}0.066 \\
(0.05)\end{array}$ & $\begin{array}{l}0.044 \\
(0.14)\end{array}$ \\
\hline pcGDP ${ }_{\text {it- } 1}$ & $\begin{array}{c}-0.000 \\
(0.00)\end{array}$ & $\begin{array}{c}-0.000 \\
(0.00)\end{array}$ \\
\hline$\Delta \mathrm{pcGDP}_{\mathrm{it}-1}$ & $\begin{array}{l}0.000 \\
(0.00)\end{array}$ & $\begin{array}{l}-0.000 \\
(0.00)\end{array}$ \\
\hline Contagion $_{\text {it }}$ & $\begin{array}{l}0.030 \\
(0.04)\end{array}$ & $\begin{array}{l}0.031 \\
(0.04)\end{array}$ \\
\hline PastDem $_{\text {it }}$ & $\begin{array}{c}1.436^{* * *} \\
(0.44)\end{array}$ & $\begin{array}{c}1.567 * * * \\
(0.43)\end{array}$ \\
\hline RegConflict $_{\mathrm{it}}$ & $\begin{array}{c}-0.278 \\
(0.17)\end{array}$ & $\begin{array}{c}-0.293^{*} \\
(0.17)\end{array}$ \\
\hline IntViolence $_{\text {it-1 }}$ & $\begin{array}{c}2.239^{* * *} * \\
(0.50)\end{array}$ & $\begin{array}{c}2.223^{* * *} \\
(0.49)\end{array}$ \\
\hline MilRegit $_{i t}$ & $\begin{array}{c}-2.184 * * * \\
(0.64)\end{array}$ & $\begin{array}{c}-2.261^{* * *} \\
(0.66)\end{array}$ \\
\hline Indepit & $\begin{array}{c}0.009 * * \\
(0.00)\end{array}$ & $\begin{array}{c}0.011^{* *} \\
(0.00)\end{array}$ \\
\hline Constant & $\begin{array}{c}-6.387^{* * *} \\
(0.56)\end{array}$ & $\begin{array}{c}-6.416^{* * *} \\
(0.65)\end{array}$ \\
\hline $\mathrm{N}$ & 2552 & 2553 \\
\hline Log Likelihood & -204.812 & -208.697 \\
\hline $\mathrm{X}^{2}$ & 85.377 & 63.183 \\
\hline Prob $>X^{2}$ & 0.000 & 0.000 \\
\hline Pseudo- $\mathrm{R}^{2}$ & 0.193 & 0.178 \\
\hline
\end{tabular}




\begin{tabular}{|c|c|c|}
\hline \multicolumn{3}{|c|}{ Reich 2002} \\
\hline & Model 1 & Model 3 \\
\hline IOScore $_{\mathrm{it}-1}$ & $\begin{array}{c}0.062^{* *} \\
(0.03)\end{array}$ & $\begin{array}{c}0.101 * * * \\
(0.04)\end{array}$ \\
\hline$\Delta \mathrm{IOS}_{\text {core }} \mathrm{it-1}$ & $\begin{array}{l}0.007 \\
(0.04)\end{array}$ & $\begin{array}{c}-0.184 \\
(0.20)\end{array}$ \\
\hline $\mathrm{pcGDP}_{\mathrm{it}-1}$ & $\begin{array}{l}-0.000 \\
(0.00)\end{array}$ & $\begin{array}{c}-0.000 \\
(0.00)\end{array}$ \\
\hline$\Delta \mathrm{pcGDP}_{\mathrm{it}-1}$ & $\begin{array}{c}-0.000 \\
(0.00)\end{array}$ & $\begin{array}{r}-0.000 \\
(0.00)\end{array}$ \\
\hline Contagion $_{\text {it }}$ & $\begin{array}{l}0.032 \\
(0.03)\end{array}$ & $\begin{array}{l}0.037 \\
(0.03)\end{array}$ \\
\hline PastDem $_{\text {it }}$ & $\begin{array}{c}1.136^{* * *} \\
(0.33)\end{array}$ & $\begin{array}{c}1.214^{* * * *} \\
(0.33)\end{array}$ \\
\hline RegConflict $_{i t}$ & $\begin{array}{c}-0.452^{* * *} \\
(0.17)\end{array}$ & $\begin{array}{c}-0.461 * * * \\
(0.18)\end{array}$ \\
\hline IntViolence $_{i t-1}$ & $\begin{array}{c}1.456^{* * * *} \\
(0.38)\end{array}$ & $\begin{array}{c}1.436 * * * \\
(0.37)\end{array}$ \\
\hline MilReg $_{i t}$ & $\begin{array}{c}-0.971^{* *} \\
(0.44)\end{array}$ & $\begin{array}{c}-0.944 * * \\
(0.45)\end{array}$ \\
\hline Indep $_{i t}$ & $\begin{array}{l}0.002 \\
(0.00)\end{array}$ & $\begin{array}{l}0.002 \\
(0.00)\end{array}$ \\
\hline Constant & $\begin{array}{c}-5.086^{* * *} \\
(0.49)\end{array}$ & $\begin{array}{c}-5.817 * * * \\
(0.62)\end{array}$ \\
\hline $\mathrm{N}$ & 2309 & 2310 \\
\hline Log Likelihood & -239.775 & -238.608 \\
\hline $\mathrm{X}^{2}$ & 47.353 & 44.927 \\
\hline Prob $>X^{2}$ & 0.000 & 0.000 \\
\hline Pseudo-R ${ }^{2}$ & 0.103 & 0.107 \\
\hline $\begin{array}{l}{ }^{*} \mathrm{p}<0.10,{ }^{* *} \\
\text { including non-1 }\end{array}$ & $\begin{array}{l}5, * * * \mathrm{p}<0 . \\
\text { al organizat }\end{array}$ & $\begin{array}{l}\text { Model 3: } \\
s \text { and IFIs }\end{array}$ \\
\hline
\end{tabular}

\begin{tabular}{|c|c|c|}
\hline \multicolumn{3}{|c|}{ Reich 2002: HYB } \\
\hline & Model 1 & Model 3 \\
\hline \multirow[t]{2}{*}{ IOScore $_{\mathrm{it}-1}$} & 0.027 & 0.030 \\
\hline & $(0.04)$ & $(0.04)$ \\
\hline \multirow[t]{2}{*}{$\Delta$ IOScore $_{\mathrm{it}-1}$} & 0.065 & -0.341 \\
\hline & $(0.05)$ & $(0.21)$ \\
\hline \multirow[t]{2}{*}{$\mathrm{pcGDP}_{\mathrm{it}-1}$} & -0.000 & -0.000 \\
\hline & $(0.00)$ & $(0.00)$ \\
\hline \multirow[t]{2}{*}{$\Delta \mathrm{pcGDP}$ it-1 } & $-0.001 * *$ & -0.001 \\
\hline & $(0.00)$ & $(0.00)$ \\
\hline \multirow[t]{2}{*}{ Contagion $_{\text {it }}$} & $0.067^{*}$ & $0.074 *$ \\
\hline & $(0.04)$ & $(0.04)$ \\
\hline \multirow[t]{2}{*}{ PastDem $_{\text {it }}$} & $2.534 * * *$ & $2.661 * * *$ \\
\hline & $(0.55)$ & $(0.51)$ \\
\hline \multirow[t]{2}{*}{ RegConflict $_{\text {it }}$} & $-0.679 *$ & $-0.696^{*}$ \\
\hline & $(0.35)$ & $(0.37)$ \\
\hline \multirow[t]{2}{*}{ IntViolence $_{\mathrm{it}-1}$} & $1.225^{* *}$ & $1.138^{*}$ \\
\hline & $(0.59)$ & $(0.60)$ \\
\hline \multirow[t]{2}{*}{ MilReg $_{\text {it }}$} & $-2.139 * * *$ & $-2.178 * * *$ \\
\hline & $(0.72)$ & $(0.71)$ \\
\hline \multirow[t]{2}{*}{ Indepit $_{\text {it }}$} & 0.005 & 0.006 \\
\hline & $(0.00)$ & $(0.00)$ \\
\hline \multirow[t]{2}{*}{ Constant } & $-6.130 * * *$ & $-6.338 * * *$ \\
\hline & $(0.69)$ & $(0.77)$ \\
\hline $\mathrm{N}$ & 2533 & 2534 \\
\hline Log Likelihood & -109.570 & -108.794 \\
\hline $\mathrm{X}^{2}$ & 70.044 & 75.330 \\
\hline Prob $>X^{2}$ & 0.000 & 0.000 \\
\hline Pseudo-R ${ }^{2}$ & 0.164 & 0.170 \\
\hline \multicolumn{3}{|c|}{$\begin{array}{l}\quad * \mathrm{p}<0.10,{ }^{* *} \mathrm{p}<0.05,{ }^{* * *} \mathrm{p}<0.01 . \text { Model } 3: \\
\text { including non-regional organizations and IFIs }\end{array}$} \\
\hline
\end{tabular}

\begin{tabular}{|c|c|c|}
\hline \multicolumn{3}{|c|}{ Svolik 2012} \\
\hline & Mode1 1 & Mode1 3 \\
\hline \multirow[t]{2}{*}{ IOScore $_{i t-1}$} & $0.069 * *$ & 0.070 \\
\hline & $(0.03)$ & $(0.05)$ \\
\hline \multirow[t]{2}{*}{$\Delta$ IOScore $_{\mathrm{it}-1}$} & 0.038 & 0.027 \\
\hline & $(0.08)$ & $(0.17)$ \\
\hline \multirow[t]{2}{*}{ pcGDP $P_{i t-1}$} & -0.000 & -0.000 \\
\hline & $(0.00)$ & $(0.00)$ \\
\hline \multirow[t]{2}{*}{$\Delta \mathrm{pcGDP} \mathrm{it}_{\mathrm{t}-1}$} & -0.000 & -0.000 \\
\hline & $(0.00)$ & $(0.00)$ \\
\hline \multirow[t]{2}{*}{ Contagion $_{\mathrm{it}}$} & 0.037 & 0.040 \\
\hline & $(0.04)$ & $(0.04)$ \\
\hline \multirow[t]{2}{*}{ PastDem $_{\text {it }}$} & $1.770 * * *$ & $1.889 * * *$ \\
\hline & $(0.48)$ & $(0.46)$ \\
\hline \multirow[t]{2}{*}{ RegConflict $_{\text {it }}$} & -0.374 & -0.383 \\
\hline & $(0.25)$ & $(0.24)$ \\
\hline \multirow[t]{2}{*}{ IntViolence $_{\text {it }-1}$} & $1.558^{* * *}$ & $1.557 * * *$ \\
\hline & $(0.45)$ & $(0.45)$ \\
\hline \multirow[t]{2}{*}{ MilRegit $_{i t}$} & $-2.838^{* * *}$ & $-2.911 * * *$ \\
\hline & $(0.89)$ & $(0.90)$ \\
\hline \multirow[t]{2}{*}{ Indepit } & $0.011^{* *}$ & $0.012^{* *}$ \\
\hline & $(0.01)$ & $(0.01)$ \\
\hline \multirow[t]{2}{*}{ Constant } & $-6.166^{* * *}$ & $-6.465^{* * *}$ \\
\hline & $(0.58)$ & $(0.73)$ \\
\hline $\mathrm{N}$ & 2507 & 2508 \\
\hline Log Likelihood & -175.119 & -176.859 \\
\hline $\mathrm{X}^{2}$ & 84.646 & 66.137 \\
\hline Prob $>X^{2}$ & 0.000 & 0.000 \\
\hline Pseudo- $\mathrm{R}^{2}$ & 0.195 & 0.187 \\
\hline \multicolumn{3}{|c|}{$\begin{array}{l}\quad * \mathrm{p}<0.10,{ }^{* *} \mathrm{p}<0.05,{ }^{* * *} \mathrm{p}<0.01 . \text { Model } 3: \\
\text { including non-regional organizations and IFIs }\end{array}$} \\
\hline
\end{tabular}




\begin{tabular}{|c|c|c|c|c|c|}
\hline \multicolumn{3}{|c|}{ Ulfelder 2012} & \multicolumn{3}{|c|}{ Wahman et al. 2013} \\
\hline & Mode1 1 & Model 3 & & Model 1 & Mode1 3 \\
\hline IOScore $_{i t-1}$ & $\begin{array}{c}0.050^{*} \\
(0.03)\end{array}$ & $\begin{array}{l}0.045 \\
(0.05)\end{array}$ & IOScore $_{\text {it }-1}$ & $\begin{array}{l}0.049 \\
(0.04)\end{array}$ & $\begin{array}{l}0.042 \\
(0.05)\end{array}$ \\
\hline$\Delta$ IOScore $_{\mathrm{it}-1}$ & $\begin{array}{l}0.046 \\
(0.05)\end{array}$ & $\begin{array}{c}-0.269 \\
(0.26)\end{array}$ & $\Delta$ IOScore $_{\text {it }-1}$ & $\begin{array}{l}0.058 \\
(0.06)\end{array}$ & $\begin{array}{c}-0.473 \\
(0.38)\end{array}$ \\
\hline pcGDP $P_{\text {it }-1}$ & $\begin{array}{c}-0.000 \\
(0.00)\end{array}$ & $\begin{array}{c}-0.000 \\
(0.00)\end{array}$ & pcGDP $P_{\text {it }-1}$ & $\begin{array}{c}-0.000 \\
(0.00)\end{array}$ & $\begin{array}{c}-0.000 \\
(0.00)\end{array}$ \\
\hline$\Delta p c G D P_{i t-1}$ & $\begin{array}{r}-0.001 \\
(0.00)\end{array}$ & $\begin{array}{r}-0.000 \\
(0.00)\end{array}$ & $\Delta \mathrm{pcGDP}$ it-1 & $\begin{array}{l}0.000 \\
(0.00)\end{array}$ & $\begin{array}{l}0.000 \\
(0.00)\end{array}$ \\
\hline Contagion $_{\text {it }}$ & $\begin{array}{l}0.028 \\
(0.04)\end{array}$ & $\begin{array}{l}0.032 \\
(0.04)\end{array}$ & Contagion $_{\text {it }}$ & $\begin{array}{c}0.069^{*} \\
(0.04)\end{array}$ & $\begin{array}{c}0.083^{* *} \\
(0.04)\end{array}$ \\
\hline PastDem $_{\text {it }}$ & $\begin{array}{c}1.646^{* * *} \\
(0.40)\end{array}$ & $\begin{array}{c}1.785^{* * *} \\
(0.38)\end{array}$ & PastDem $_{\text {it }}$ & $\begin{array}{c}2.145^{* * *} \\
(0.50)\end{array}$ & $\begin{array}{c}2.251 * * * \\
(0.54)\end{array}$ \\
\hline RegConflict $_{\text {it }}$ & $\begin{array}{c}-0.241 \\
(0.16)\end{array}$ & $\begin{array}{c}-0.260 \\
(0.16)\end{array}$ & RegConflict $_{\text {it }}$ & $\begin{array}{c}-0.668^{* *} \\
(0.34)\end{array}$ & $\begin{array}{c}-0.695^{* *} \\
(0.34)\end{array}$ \\
\hline IntViolence $_{\text {it- } 1}$ & $\begin{array}{c}1.559 * * * \\
(0.40)\end{array}$ & $\begin{array}{c}1.524^{* * *} \\
(0.40)\end{array}$ & IntViolence $\mathrm{it}_{\mathrm{t}-1}$ & $\begin{array}{c}1.759 * * * \\
(0.52)\end{array}$ & $\begin{array}{c}1.673 * * * \\
(0.51)\end{array}$ \\
\hline MilReg $_{\text {it }}$ & $\begin{array}{c}-2.606^{* * *} \\
(0.81)\end{array}$ & $\begin{array}{c}-2.661 * * * \\
(0.85)\end{array}$ & MilRegit $_{\text {it }}$ & $\begin{array}{c}-3.729 * * * \\
(0.84)\end{array}$ & $\begin{array}{c}-3.791 * * * \\
(0.86)\end{array}$ \\
\hline Indepit & $\begin{array}{c}0.012^{* *} \\
(0.00)\end{array}$ & $\begin{array}{c}0.013^{* * *} \\
(0.00)\end{array}$ & Indepit & $\begin{array}{c}0.010^{* *} \\
(0.00)\end{array}$ & $\begin{array}{c}0.013^{* * *} \\
(0.00)\end{array}$ \\
\hline Constant & $\begin{array}{c}-5.677 * * * \\
(0.51)\end{array}$ & $\begin{array}{c}-5.842 * * * \\
(0.65)\end{array}$ & Constant & $\begin{array}{c}-5.757 * * * \\
(0.61)\end{array}$ & $\begin{array}{c}-5.911^{* * *} \\
(0.80)\end{array}$ \\
\hline $\mathrm{N}$ & 2436 & 2436 & $\mathrm{~N}$ & 1589 & 1589 \\
\hline Log Likelihood & -199.846 & -200.137 & Log Likelihood & -124.929 & -123.762 \\
\hline $\mathrm{X}^{2}$ & 73.690 & 87.448 & $\mathrm{X}^{2}$ & 71.663 & 61.878 \\
\hline Prob $>X^{2}$ & 0.000 & 0.000 & Prob $>X^{2}$ & 0.000 & 0.000 \\
\hline Pseudo-R ${ }^{2}$ & 0.180 & 0.179 & Pseudo-R ${ }^{2}$ & 0.257 & 0.264 \\
\hline $\begin{array}{l}{ }^{*} \mathrm{p}<0.10,{ }^{* *} \\
\text { including non-1 }\end{array}$ & $\begin{array}{l}\mathrm{p}, * * * \mathrm{p}<0 . \\
\text { al organizat }\end{array}$ & $\begin{array}{l}\text { Model 3: } \\
\text { s and IFIs }\end{array}$ & $\begin{array}{l}{ }^{*} \mathrm{p}<0.10,{ }^{* *} \\
\text { including non- }\end{array}$ & $\begin{array}{l}5, * * * \mathrm{p}<0 \\
\text { al organiza }\end{array}$ & $\begin{array}{l}\text { Model 3: } \\
\text { is and IFIs }\end{array}$ \\
\hline
\end{tabular}




\section{Appendix V: Robustness Checks: Donno 2013}

\begin{tabular}{|c|c|c|}
\hline \multicolumn{3}{|c|}{ Boix et al. 2013} \\
\hline & Model 3 & Model 5 \\
\hline \multirow{3}{*}{$\begin{array}{l}\text { Competitive Authoritarian } \\
\text { Regime (CAR) }\end{array}$} & -0.3806 & $-1.2232 *$ \\
\hline & $(0.4692)$ & $(0.6816)$ \\
\hline & $-4.1178^{* * *}$ & $-4.6046 * * *$ \\
\hline \multirow[t]{2}{*}{ Opposition Coalition } & $(0.3498)$ & $(0.6374)$ \\
\hline & $4.7133^{* * *}$ & $6.7091 * * *$ \\
\hline \multirow[t]{2}{*}{ CAR * Opp. Coalition } & $(0.7758)$ & $(1.1145)$ \\
\hline & $-4.8555^{* * *}$ & $-10.0136^{* * *}$ \\
\hline \multirow[t]{2}{*}{ International Conditionality } & $(0.5935)$ & (1.3695) \\
\hline & $5.0154 * * *$ & $11.5480^{* * *}$ \\
\hline \multirow[t]{2}{*}{ CAR $*$ Conditionality } & $(1.1086)$ & $(1.6645)$ \\
\hline & 0.0000 & 0.0000 \\
\hline \multirow[t]{2}{*}{ Main Election } & $()$. & $()$. \\
\hline & $-1.0790^{* * *}$ & $-2.4780 * * *$ \\
\hline \multirow[t]{2}{*}{ Incumbent running } & $(0.3988)$ & $(0.8941)$ \\
\hline & 0.0438 & $-0.1498 * *$ \\
\hline \multirow[t]{2}{*}{ \# Previous elections } & $(0.0412)$ & $(0.0726)$ \\
\hline & -0.4149 & -0.3513 \\
\hline \multirow[t]{2}{*}{ GDP per capita (lagged 1 year) } & $(0.2630)$ & $(0.3488)$ \\
\hline & -0.0182 & $0.1212 *$ \\
\hline \multirow[t]{2}{*}{ GDP growth (lagged 1 year) } & $(0.0392)$ & $(0.0672)$ \\
\hline & 0.0000 & 0.0000 \\
\hline \multirow[t]{2}{*}{ Central and Eastern Europe } & (.) & (.) \\
\hline & $1.2513^{*}$ & $4.0677^{* * *}$ \\
\hline \multirow[t]{2}{*}{ Americas } & $(0.7472)$ & $(1.2917)$ \\
\hline & & $7.4721 * * *$ \\
\hline \multirow[t]{2}{*}{ Alternation, Previous Elec. } & & $(0.5948)$ \\
\hline & & $-3.1344 * * *$ \\
\hline \multirow[t]{2}{*}{ Misconduct Intensity } & & $(0.9188)$ \\
\hline & & $0.8238^{* *}$ \\
\hline \multirow[t]{2}{*}{ Prior Liberalization } & & $(0.3839)$ \\
\hline & & -0.4232 \\
\hline Regime Openness & & $(0.4520)$ \\
\hline \multirow[t]{2}{*}{ Constant } & 1.7530 & $6.0051^{* *}$ \\
\hline & $(1.8799)$ & $(2.9161)$ \\
\hline $\mathrm{N}$ & 92 & 92 \\
\hline Pseudo-R2 & 0.235 & 0.565 \\
\hline Log Likelihood & -22.546 & -12.819 \\
\hline
\end{tabular}

\begin{tabular}{|c|c|c|}
\hline \multicolumn{3}{|c|}{ FH: free, FIW } \\
\hline & Model 3 & Model 5 \\
\hline \multirow{3}{*}{$\begin{array}{l}\text { Competitive Authoritarian Regime } \\
\text { (CAR) }\end{array}$} & -0.5328 & -31.3667 \\
\hline & $(0.6369)$ & (.) \\
\hline & 0.0000 & 0.0000 \\
\hline \multirow[t]{2}{*}{ Opposition Coalition } & (.) & $()$. \\
\hline & 0.0000 & 0.0000 \\
\hline \multirow[t]{2}{*}{ CAR * Opp. Coalition } & (.) & $()$. \\
\hline & $-5.1090^{* * *}$ & -12.2400 \\
\hline \multirow[t]{2}{*}{ International Conditionality } & $(0.7091)$ & $()$. \\
\hline & $6.6558 * * *$ & 203.2266 \\
\hline \multirow[t]{2}{*}{ CAR $*$ Conditionality } & $(1.0801)$ & $()$. \\
\hline & 0.0000 & 0.0000 \\
\hline \multirow[t]{2}{*}{ Main Election } & (.) & (.) \\
\hline & $-1.5014^{* * *}$ & -118.4691 \\
\hline \multirow[t]{2}{*}{ Incumbent running } & $(0.4835)$ & $()$. \\
\hline & $0.1725^{* * *}$ & 10.7278 \\
\hline \multirow[t]{2}{*}{ \# Previous elections } & $(0.0662)$ & $()$. \\
\hline & $-0.9531 * *$ & -91.6948 \\
\hline \multirow[t]{2}{*}{ GDP per capita (lagged 1 year) } & $(0.3946)$ & $()$. \\
\hline & 0.0638 & 9.7749 \\
\hline \multirow[t]{2}{*}{ GDP growth (lagged 1 year) } & $(0.0816)$ & $()$. \\
\hline & 0.0000 & 0.0000 \\
\hline \multirow[t]{2}{*}{ Central and Eastern Europe } & $()$. & $()$. \\
\hline & $1.7829 * *$ & 263.1907 \\
\hline Americas & $(0.8223)$ & $\begin{array}{c}(.) \\
0.0000\end{array}$ \\
\hline Alternation, Previous Elec. & & $\begin{array}{c}(.) \\
-101.8379\end{array}$ \\
\hline Misconduct Intensity & & $\begin{array}{c}(.) \\
21.3266\end{array}$ \\
\hline Prior Liberalization & & $\begin{array}{c}(.) \\
-53.7516\end{array}$ \\
\hline Regime Openness & & $()$. \\
\hline \multirow[t]{2}{*}{ Constant } & 3.3470 & 642.6951 \\
\hline & $(2.2857)$ & $()$. \\
\hline $\mathrm{N}$ & 77 & 75 \\
\hline Pseudo-R2 & 0.318 & 1.000 \\
\hline Log Likelihood & -10.731 & 0.000 \\
\hline
\end{tabular}




\begin{tabular}{|c|c|c|}
\hline \multicolumn{3}{|c|}{ FH: partly free, FIW } \\
\hline & Model 3 & Model 5 \\
\hline \multirow{3}{*}{$\begin{array}{l}\text { Competitive Authoritarian Regime } \\
\text { (CAR) }\end{array}$} & -0.0424 & 0.0187 \\
\hline & $(0.4540)$ & $(0.4944)$ \\
\hline & $-3.8412 * * *$ & $-3.9728 * * *$ \\
\hline \multirow[t]{2}{*}{ Opposition Coalition } & $(0.4780)$ & $(0.4693)$ \\
\hline & $4.3555^{* * *}$ & $4.6558^{* * *}$ \\
\hline \multirow[t]{2}{*}{ CAR $*$ Opp. Coalition } & $(0.7397)$ & $(0.7964)$ \\
\hline & 0.0000 & 0.0000 \\
\hline \multirow[t]{2}{*}{ International Conditionality } & $()$. & $()$. \\
\hline & 0.0000 & 0.0000 \\
\hline \multirow[t]{2}{*}{ CAR $*$ Conditionality } & $()$. & $()$. \\
\hline & 0.2372 & 0.5636 \\
\hline \multirow[t]{2}{*}{ Main Election } & $(0.4346)$ & $(0.4892)$ \\
\hline & -0.6128 & $-0.8257^{*}$ \\
\hline \multirow[t]{2}{*}{ Incumbent running } & $(0.4335)$ & $(0.4425)$ \\
\hline & -0.0003 & -0.0326 \\
\hline \multirow[t]{2}{*}{ \# Previous elections } & $(0.0453)$ & $(0.0487)$ \\
\hline & $-0.6586^{* * *}$ & $-0.6227 * *$ \\
\hline \multirow[t]{2}{*}{ GDP per capita (lagged 1 year) } & $(0.2438)$ & $(0.2596)$ \\
\hline & 0.0078 & 0.0206 \\
\hline \multirow[t]{2}{*}{ GDP growth (lagged 1 year) } & $(0.0182)$ & $(0.0196)$ \\
\hline & 0.0000 & 0.0000 \\
\hline \multirow[t]{2}{*}{ Central and Eastern Europe } & (.) & (.) \\
\hline & 0.0000 & 0.0000 \\
\hline Americas & $()$. & (.) \\
\hline \multirow[t]{2}{*}{ Alternation, Previous Elec. } & & $()$. \\
\hline & & -0.3457 \\
\hline \multirow[t]{2}{*}{ Misconduct Intensity } & & $(0.2313)$ \\
\hline & & 0.0535 \\
\hline \multirow[t]{2}{*}{ Prior Liberalization } & & $(0.1160)$ \\
\hline & & $-0.5613^{* *}$ \\
\hline Regime Openness & & $(0.2519)$ \\
\hline \multirow[t]{2}{*}{ Constant } & 2.6856 & $4.5059^{* *}$ \\
\hline & $(1.6393)$ & $(1.9602)$ \\
\hline $\mathrm{N}$ & 144 & 142 \\
\hline Pseudo-R2 & 0.181 & 0.261 \\
\hline Log Likelihood & -31.823 & -28.590 \\
\hline
\end{tabular}

\begin{tabular}{|c|c|c|}
\hline \multicolumn{3}{|c|}{ FH: free, PR } \\
\hline & Model 3 & Model 5 \\
\hline Competitive Authoritarian Regime & -0.2587 & -16.8659 \\
\hline (CAR) & $\begin{array}{c}(0.6444) \\
0.0000\end{array}$ & $\begin{array}{c}(.) \\
0.0000\end{array}$ \\
\hline Opposition Coalition & $()$. & $()$. \\
\hline & 0.0000 & 0.0000 \\
\hline CAR $*$ Opp. Coalition & (.) & $()$. \\
\hline & $-5.3405^{* * *}$ & -73.2702 \\
\hline International Conditionality & $(0.7861)$ & (.) \\
\hline & $6.8312^{* * *}$ & 200.8569 \\
\hline CAR $*$ Conditionality & $(1.1025)$ & (.) \\
\hline & 0.0000 & 0.0000 \\
\hline Main Election & (.) & $()$. \\
\hline & $-1.8898^{* * *}$ & -72.1978 \\
\hline Incumbent running & $(0.6270)$ & (.) \\
\hline & $0.2188^{* * *}$ & 7.8973 \\
\hline \# Previous elections & $(0.0838)$ & (.) \\
\hline & $-1.3584^{* *}$ & -63.6061 \\
\hline GDP per capita (lagged 1 year) & $(0.5297)$ & $()$. \\
\hline & 0.0085 & 6.6888 \\
\hline GDP growth (lagged 1 year) & $(0.0637)$ & $()$. \\
\hline & 0.0000 & 0.0000 \\
\hline Central and Eastern Europe & $\begin{array}{c}(.) \\
1.8440 * *\end{array}$ & $\begin{array}{c}(.) \\
173.3312\end{array}$ \\
\hline Americas & $(0.9039)$ & $\begin{array}{c}(.) \\
71.6246\end{array}$ \\
\hline Alternation, Previous Elec. & & $\begin{array}{c}(.) \\
-65.9759\end{array}$ \\
\hline Misconduct Intensity & & $\begin{array}{c}(.) \\
13.0388\end{array}$ \\
\hline Prior Liberalization & & $\begin{array}{c}(.) \\
-33.3844\end{array}$ \\
\hline Regime Openness & & (.) \\
\hline Constant & $\begin{array}{l}5.9028^{*} \\
(3.0246\end{array}$ & 422.1852 \\
\hline $\mathrm{N}$ & $\begin{array}{c}(3.0240) \\
77\end{array}$ & $\begin{array}{l}(\cdot) \\
77\end{array}$ \\
\hline Pseudo-R2 & 0.371 & 1.000 \\
\hline Log Likelihood & -11.638 & 0.000 \\
\hline
\end{tabular}

\begin{tabular}{|c|c|c|}
\hline \multicolumn{3}{|c|}{ FH: partly free, PR } \\
\hline & Model 3 & Model 5 \\
\hline Competitive Authoritarian Regime & -0.2359 & -0.2431 \\
\hline (CAR) & $(0.4073)$ & $(0.4644)$ \\
\hline & $-4.1556^{* * *}$ & $-4.3760^{* * *}$ \\
\hline Opposition Coalition & $(0.3566)$ & $(0.4405)$ \\
\hline & $4.6137 * * *$ & $5.0183^{* * *}$ \\
\hline CAR * Opp. Coalition & $(0.6456)$ & $(0.7114)$ \\
\hline & 0.0000 & 0.0000 \\
\hline International Conditionality & (.) & (.) \\
\hline & 0.0000 & 0.0000 \\
\hline CAR $*$ Conditionality & $()$. & $()$. \\
\hline & 0.4409 & $0.7044^{*}$ \\
\hline Main Election & $(0.3718)$ & $(0.4144)$ \\
\hline & $-0.8299 * *$ & $-0.9498 * *$ \\
\hline Incumbent running & $(0.3833)$ & $(0.3974)$ \\
\hline & 0.0051 & -0.0412 \\
\hline \# Previous elections & $(0.0395)$ & $(0.0432)$ \\
\hline & $-0.4497 * *$ & $-0.3815^{* *}$ \\
\hline GDP per capita (lagged 1 year) & $(0.1905)$ & $(0.1728)$ \\
\hline & -0.0113 & 0.0026 \\
\hline GDP growth (lagged 1 year) & $(0.0230)$ & $(0.0259)$ \\
\hline & 0.0000 & 0.0000 \\
\hline Central and Eastern Europe & (.) & (.) \\
\hline Americas & $()$. & $\begin{array}{c}(.) \\
0.0000\end{array}$ \\
\hline Alternation, Previous Elec. & & $\begin{array}{c}(.) \\
-0.5963^{* *}\end{array}$ \\
\hline Misconduct Intensity & & $\begin{array}{c}(0.2497) \\
-0.0379\end{array}$ \\
\hline Prior Liberalization & & $\begin{array}{c}(0.1484) \\
-0.3913^{*}\end{array}$ \\
\hline Regime Openness & & $(0.2101)$ \\
\hline Constant & $\begin{array}{c}1.6757 \\
(1.3014)\end{array}$ & $\begin{array}{c}3.1483^{* *} \\
(1.4786)\end{array}$ \\
\hline $\mathrm{N}$ & 144 & 142 \\
\hline Pseudo-R2 & 0.137 & 0.249 \\
\hline Log Likelihood & -37.666 & -32.631 \\
\hline
\end{tabular}




\begin{tabular}{|l|c|}
\hline \multicolumn{2}{|c|}{ FH: HYB, FIW } \\
\hline & Model 3 \\
\hline
\end{tabular}

gdpgrowth_lag1 $>6.7$ predicts $\quad$ gdpgrowth_lag1 $>6.7$ predicts data perfectly

\begin{tabular}{|l|c|}
\hline \multicolumn{2}{|c|}{ FH: HYB, PR } \\
\hline & Model 3 \\
\hline
\end{tabular}

gdpgrowth_lag1 $>6.7$ predicts

data perfectly gdpgrowth_lag1 $>6.7$ predicts data perfectly

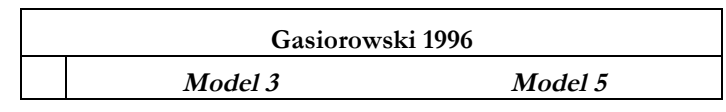

bm_incrun_fill $<=0$ predicts data perfectly

bm_incrun_fill $<=0$ predicts data perfectly 


\begin{tabular}{|l|c|}
\hline \multicolumn{2}{|c|}{ Gasiorowski 1996: HYB } \\
\hline & Model 3 \\
\hline
\end{tabular}

bm_incrun_fill $>0$ predicts $\quad$ om_incrun_fill $>0$ predicts data perfectly data perfectly

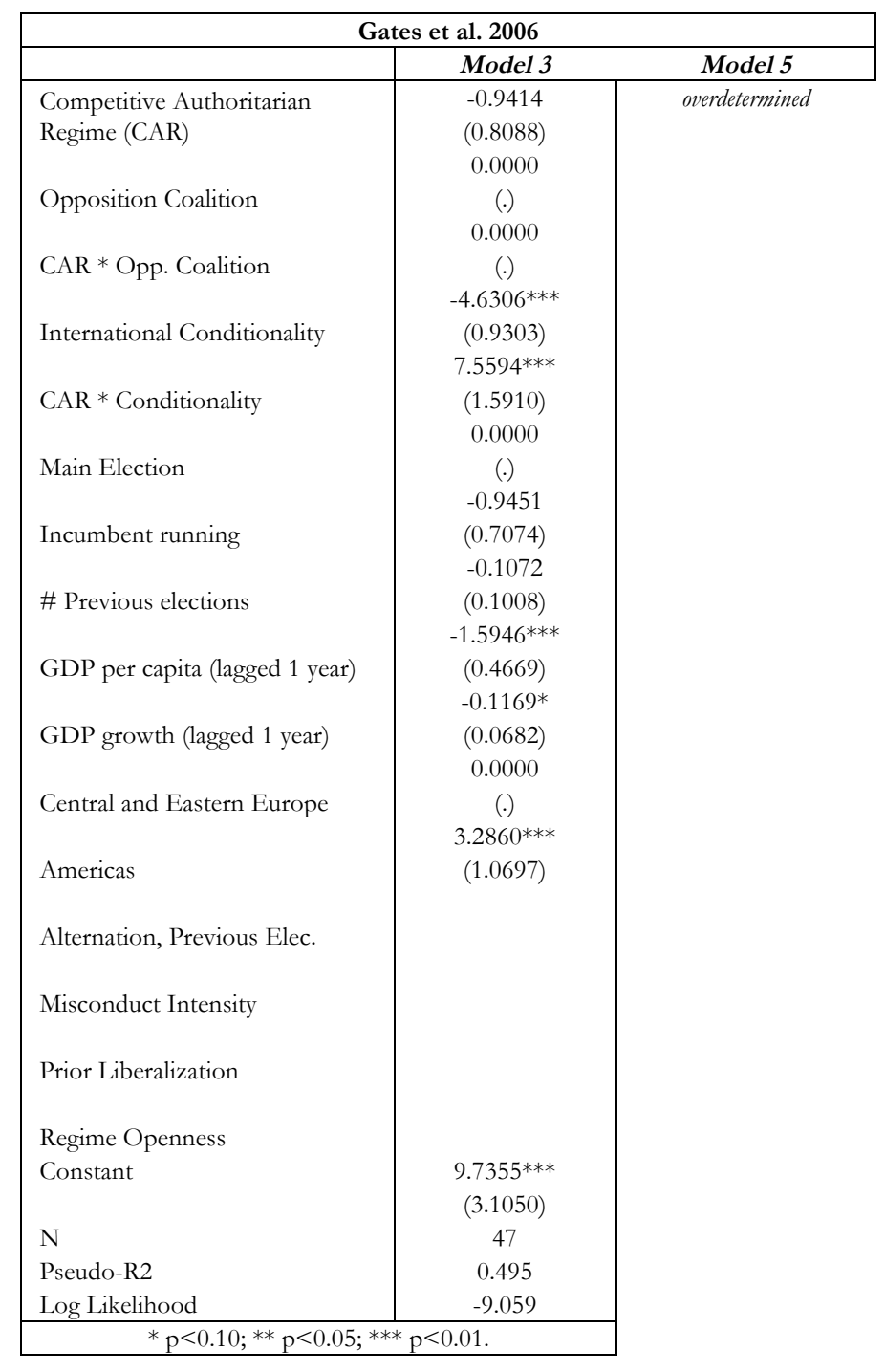

\begin{tabular}{|c|c|c|}
\hline \multicolumn{3}{|c|}{ Geddes et al. 2014} \\
\hline & Mode1 3 & Model 5 \\
\hline Competitive Authoritarian & 0.0826 & \multirow{28}{*}{ convergence not acbieved } \\
\hline Regime (CAR) & $\begin{array}{c}(0.5866) \\
-4.2539 * * *\end{array}$ & \\
\hline \multirow[t]{2}{*}{ Opposition Coalition } & $(0.3782)$ & \\
\hline & $5.0252^{* * *}$ & \\
\hline \multirow[t]{2}{*}{ CAR * Opp. Coalition } & $(0.7732)$ & \\
\hline & $-5.0203 * * *$ & \\
\hline \multirow[t]{2}{*}{ International Conditionality } & $(0.6932)$ & \\
\hline & $4.7606^{* * *}$ & \\
\hline \multirow[t]{2}{*}{ CAR $*$ Conditionality } & $(1.2528)$ & \\
\hline & 0.0000 & \\
\hline \multirow[t]{2}{*}{ Main Election } & (.) & \\
\hline & $-1.2762^{* * *}$ & \\
\hline \multirow[t]{2}{*}{ Incumbent running } & $(0.4009)$ & \\
\hline & 0.0636 & \\
\hline \multirow[t]{2}{*}{ \# Previous elections } & $(0.0397)$ & \\
\hline & -0.4507 & \\
\hline \multirow[t]{2}{*}{ GDP per capita (lagged 1 year) } & $(0.3013)$ & \\
\hline & -0.0278 & \\
\hline \multirow[t]{2}{*}{ GDP growth (lagged 1 year) } & $(0.0429)$ & \\
\hline & 0.0000 & \\
\hline Central and Eastern Europe & (.) & \\
\hline \multirow[t]{2}{*}{ Americas } & $1.5621^{* *}$ & \\
\hline & $(0.7794)$ & \\
\hline \multirow[t]{2}{*}{ Constant } & 1.7752 & \\
\hline & (2.1500) & \\
\hline $\mathrm{N}$ & 91 & \\
\hline Pseudo-R2 & 0.351 & \\
\hline Log Likelihood & -21.770 & \\
\hline \multicolumn{2}{|c|}{$* \mathrm{p}<0.10 ; * * \mathrm{p}<0.05 ; * * * \mathrm{p}<0.01$} & \\
\hline
\end{tabular}




\begin{tabular}{|c|c|c|}
\hline \multicolumn{3}{|c|}{ Magaloni et al. 2013} \\
\hline & Model 3 & Model 5 \\
\hline $\begin{array}{l}\text { Competitive Authoritarian Regime } \\
\text { (CAR) }\end{array}$ & $\begin{array}{l}-0.3325 \\
(0.4184)\end{array}$ & $\begin{array}{l}-0.6307 \\
(0.4425) \\
3.6537 * * *\end{array}$ \\
\hline Opposition Coalition & $(0.4059)$ & $(0.6722)$ \\
\hline CAR $*$ Opp. Coalition & $\begin{array}{c}5.0548 * * * \\
(0.7099) \\
-47637 * * *\end{array}$ & $\begin{array}{c}5.4350^{* * * *} \\
(0.9616) \\
-6.1311 * * *\end{array}$ \\
\hline International Conditionality & $\begin{array}{c}(0.5777) \\
4.8935^{* * *}\end{array}$ & $\begin{array}{c}(1.1299) \\
6.8362 * * *\end{array}$ \\
\hline CAR * Conditionality & $\begin{array}{l}(1.0729) \\
1.4452^{* *}\end{array}$ & $\begin{array}{l}(1.4828) \\
1.7811^{*}\end{array}$ \\
\hline Main Election & $\begin{array}{l}(0.6591) \\
-0.7419 *\end{array}$ & $\begin{array}{l}(1.0271) \\
-0.7227\end{array}$ \\
\hline Incumbent running & $\begin{array}{l}(0.4168) \\
0.0749 * *\end{array}$ & $\begin{array}{c}(0.5504) \\
0.0191\end{array}$ \\
\hline \# Previous elections & $\begin{array}{l}(0.0309) \\
-0.4698^{*}\end{array}$ & $\begin{array}{l}(0.0556) \\
-0.3940 *\end{array}$ \\
\hline GDP per capita (lagged 1 year) & $\begin{array}{l}(0.2597) \\
-0.0346\end{array}$ & $\begin{array}{c}(0.2382) \\
0.0096\end{array}$ \\
\hline GDP growth (lagged 1 year) & $\begin{array}{c}(0.0316) \\
0.0000\end{array}$ & $\begin{array}{c}(0.0323) \\
0.0000\end{array}$ \\
\hline Central and Eastern Europe & $\begin{array}{c}(.) \\
1.7637^{* * *}\end{array}$ & $\begin{array}{c}(.) \\
2.7026^{* * *}\end{array}$ \\
\hline Americas & $(0.6218)$ & $\begin{array}{c}(0.6951) \\
2.6826^{* * *}\end{array}$ \\
\hline Alternation, Previous Elec. & & $\begin{array}{c}(0.7256) \\
-1.3154 * * *\end{array}$ \\
\hline Misconduct Intensity & & $\begin{array}{c}(0.4336) \\
0.2839\end{array}$ \\
\hline Prior Liberalization & & $\begin{array}{l}(0.2587) \\
-0.1322\end{array}$ \\
\hline Regime Openness & & $(0.2526)$ \\
\hline Constant & $\begin{array}{c}0.1281 \\
(1.8392)\end{array}$ & $\begin{array}{c}0.8686 \\
(1.9328)\end{array}$ \\
\hline $\mathrm{N}$ & 173 & 173 \\
\hline Pseudo-R2 & 0.342 & 0.528 \\
\hline Log Likelihood & -25.158 & -18.047 \\
\hline
\end{tabular}

\begin{tabular}{|c|c|c|}
\hline \multicolumn{3}{|c|}{ Polity IV: AN=AUT } \\
\hline & Mode1 3 & Model 5 \\
\hline \multirow{3}{*}{$\begin{array}{l}\text { Competitive Authoritarian Regime } \\
\text { (CAR) }\end{array}$} & -0.3756 & -0.3961 \\
\hline & $(0.4833)$ & $(0.5252)$ \\
\hline & $-3.7593^{* * *}$ & $-2.4852^{* * *}$ \\
\hline \multirow[t]{2}{*}{ Opposition Coalition } & $(0.4459)$ & $(0.9409)$ \\
\hline & $5.3161 * * *$ & $4.6425^{* * *}$ \\
\hline \multirow[t]{2}{*}{ CAR $*$ Opp. Coalition } & $(0.8983)$ & $(1.1400)$ \\
\hline & 0.0000 & 0.0000 \\
\hline \multirow[t]{2}{*}{ International Conditionality } & (.) & (.) \\
\hline & 0.0000 & 0.0000 \\
\hline \multirow[t]{2}{*}{ CAR $*$ Conditionality } & (.) & $()$. \\
\hline & 1.1666 & 1.5350 \\
\hline \multirow[t]{2}{*}{ Main Election } & $(0.7782)$ & $(1.0904)$ \\
\hline & -0.2017 & -0.3461 \\
\hline \multirow[t]{2}{*}{ Incumbent running } & $(0.6172)$ & $(0.6738)$ \\
\hline & $0.1060^{* *}$ & $0.0969 *$ \\
\hline \multirow[t]{2}{*}{ \# Previous elections } & $(0.0452)$ & $(0.0573)$ \\
\hline & $-0.7925^{* *}$ & $-0.9762^{* * *}$ \\
\hline \multirow[t]{2}{*}{ GDP per capita (lagged 1 year) } & $(0.3193)$ & $(0.3572)$ \\
\hline & -0.0583 & -0.0486 \\
\hline \multirow[t]{2}{*}{ GDP growth (lagged 1 year) } & $(0.0370)$ & $(0.0400)$ \\
\hline & 0.0000 & 0.0000 \\
\hline \multirow[t]{2}{*}{ Central and Eastern Europe } & (.) & (.) \\
\hline & $2.4424 * * *$ & $3.2891 * * *$ \\
\hline \multirow[t]{2}{*}{ Americas } & $(0.7024)$ & $(0.9616)$ \\
\hline & & 0.0000 \\
\hline \multirow[t]{2}{*}{ Alternation, Previous Elec. } & & (.) \\
\hline & & $-0.9583^{* *}$ \\
\hline \multirow[t]{2}{*}{ Misconduct Intensity } & & $(0.3926)$ \\
\hline & & 0.2138 \\
\hline \multirow[t]{2}{*}{ Prior Liberalization } & & $(0.2488)$ \\
\hline & & -0.1046 \\
\hline Regime Openness & & $(0.2795)$ \\
\hline \multirow{2}{*}{ Constant } & 1.7466 & 3.6119 \\
\hline & $(1.9716)$ & $(2.2031)$ \\
\hline $\mathrm{N}$ & 154 & 152 \\
\hline Pseudo-R2 & 0.394 & 0.495 \\
\hline Log Likelihood & -20.780 & -17.248 \\
\hline
\end{tabular}

\begin{tabular}{|c|c|c|}
\hline \multicolumn{3}{|c|}{ Polity IV: AN=DEM } \\
\hline & Model 3 & Model 5 \\
\hline \multirow{3}{*}{$\begin{array}{l}\text { Competitive Authoritarian Regime } \\
\text { (CAR) }\end{array}$} & $-0.6659 * *$ & $-1.0775^{* * *}$ \\
\hline & $(0.3220)$ & $(0.4013)$ \\
\hline & 0.5090 & $1.3156^{*}$ \\
\hline \multirow[t]{2}{*}{ Opposition Coalition } & $(0.6860)$ & $(0.7980)$ \\
\hline & -0.1428 & -0.3166 \\
\hline \multirow[t]{2}{*}{ CAR $*$ Opp. Coalition } & $(0.9539)$ & (1.0431) \\
\hline & 0.2849 & $-1.5283^{* *}$ \\
\hline \multirow[t]{2}{*}{ International Conditionality } & $(0.4960)$ & $(0.7263)$ \\
\hline & 0.3558 & $2.1147^{* *}$ \\
\hline \multirow[t]{2}{*}{ CAR $*$ Conditionality } & $(0.7042)$ & $(1.0659)$ \\
\hline & 0.0498 & -0.1819 \\
\hline \multirow[t]{2}{*}{ Main Election } & $(0.3672)$ & $(0.4627)$ \\
\hline & 0.0146 & $0.6496 *$ \\
\hline \multirow[t]{2}{*}{ Incumbent running } & $(0.3571)$ & $(0.3697)$ \\
\hline & 0.0335 & 0.0291 \\
\hline \multirow[t]{2}{*}{ \# Previous elections } & $(0.0372)$ & $(0.0512)$ \\
\hline & $-0.4948^{* * *}$ & $-0.5460 * *$ \\
\hline \multirow[t]{2}{*}{ GDP per capita (lagged 1 year) } & $(0.1859)$ & $(0.2494)$ \\
\hline & 0.0168 & $0.0644^{* *}$ \\
\hline \multirow[t]{2}{*}{ GDP growth (lagged 1 year) } & $(0.0226)$ & $(0.0300)$ \\
\hline & 0.0000 & 0.0000 \\
\hline \multirow[t]{2}{*}{ Central and Eastern Europe } & $()$. & $()$. \\
\hline & 1.0191 & $1.8600^{* *}$ \\
\hline \multirow[t]{2}{*}{ Americas } & $(0.6397)$ & $(0.9443)$ \\
\hline & & $2.3504^{* * *}$ \\
\hline \multirow[t]{2}{*}{ Alternation, Previous Elec. } & & $(0.6850)$ \\
\hline & & -0.0967 \\
\hline \multirow[t]{2}{*}{ Misconduct Intensity } & & $(0.2344)$ \\
\hline & & $-0.5855^{* * *}$ \\
\hline \multirow[t]{2}{*}{ Prior Liberalization } & & $(0.1601)$ \\
\hline & & $-0.7089 * *$ \\
\hline Regime Openness & & $(0.3211)$ \\
\hline \multirow[t]{2}{*}{ Constant } & 1.4052 & 3.1517 \\
\hline & $(1.2110)$ & $(2.2275)$ \\
\hline $\mathrm{N}$ & 173 & 173 \\
\hline Pseudo-R2 & 0.129 & 0.400 \\
\hline Log Likelihood & -33.288 & -22.931 \\
\hline
\end{tabular}




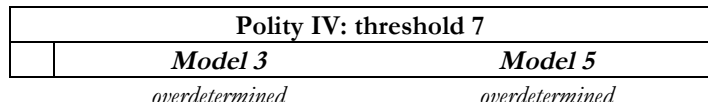

overdetermined overdetermined

\begin{tabular}{|c|c|c|}
\hline \multicolumn{3}{|c|}{ Polity IV: threshold 1} \\
\hline & Model 3 & Model 5 \\
\hline \multirow{3}{*}{$\begin{array}{l}\text { Competitive Authoritarian Regime } \\
\text { (CAR) }\end{array}$} & -0.1833 & -0.0989 \\
\hline & $(0.3479)$ & $(0.3303)$ \\
\hline & $-4.6030^{* * *}$ & $-4.2777 * * *$ \\
\hline \multirow[t]{2}{*}{ Opposition Coalition } & $(0.6204)$ & $(0.4918)$ \\
\hline & $\mathrm{tm}$ & $5.6920^{* * *}$ \\
\hline \multirow[t]{2}{*}{ CAR * Opp. Coalition } & $(0.7422)$ & $(0.6348)$ \\
\hline & $1.1722^{* *}$ & 1.3763 \\
\hline \multirow[t]{2}{*}{ International Conditionality } & $(0.5926)$ & $(0.8551)$ \\
\hline & -0.9772 & -0.8809 \\
\hline \multirow[t]{2}{*}{ CAR $*$ Conditionality } & $(0.8380)$ & $(1.0948)$ \\
\hline & $1.1363^{* * *}$ & $1.3906^{* * *}$ \\
\hline \multirow[t]{2}{*}{ Main Election } & $(0.3937)$ & $(0.3692)$ \\
\hline & -0.3023 & -0.3125 \\
\hline \multirow[t]{2}{*}{ Incumbent running } & $(0.3460)$ & $(0.3824)$ \\
\hline & 0.0138 & -0.0292 \\
\hline \multirow[t]{2}{*}{ \# Previous elections } & $(0.0355)$ & $(0.0408)$ \\
\hline & -0.2431 & -0.1422 \\
\hline \multirow[t]{2}{*}{ GDP per capita (lagged 1 year) } & $(0.1608)$ & $(0.1647)$ \\
\hline & 0.0088 & 0.0180 \\
\hline \multirow[t]{2}{*}{ GDP growth (lagged 1 year) } & $(0.0321)$ & $(0.0315)$ \\
\hline & 0.0000 & 0.0000 \\
\hline \multirow[t]{2}{*}{ Central and Eastern Europe } & $()$. & (.) \\
\hline & $1.0680^{* *}$ & $1.4813^{* * *}$ \\
\hline \multirow[t]{2}{*}{ Americas } & $(0.4721)$ & $(0.5472)$ \\
\hline & & 0.2495 \\
\hline \multirow[t]{2}{*}{ Alternation, Previous Elec. } & & $(0.8990)$ \\
\hline & & $-0.6512 * * *$ \\
\hline \multirow[t]{2}{*}{ Misconduct Intensity } & & $(0.2014)$ \\
\hline & & 0.0790 \\
\hline \multirow[t]{2}{*}{ Prior Liberalization } & & (0.1413) \\
\hline & & -0.3613 \\
\hline Regime Openness & & $(0.2529)$ \\
\hline \multirow[t]{2}{*}{ Constant } & -0.6365 & 0.4611 \\
\hline & (1.1107) & (1.3944) \\
\hline $\mathrm{N}$ & 173 & 173 \\
\hline Pseudo-R2 & 0.260 & 0.337 \\
\hline Log Likelihood & -42.768 & -38.286 \\
\hline
\end{tabular}

\begin{tabular}{|c|c|c|}
\hline \multicolumn{3}{|c|}{ Polity IV: exrec \& parcomp } \\
\hline & Model 3 & Model 5 \\
\hline \multirow{3}{*}{$\begin{array}{l}\text { Competitive Authoritarian Regime } \\
\text { (CAR) }\end{array}$} & $\begin{array}{c}-0.4707 \\
\end{array}$ & -0.3676 \\
\hline & $(0.3542)$ & $(0.3577)$ \\
\hline & $-4.1179 * * *$ & $-4.8795^{* * *}$ \\
\hline \multirow[t]{2}{*}{ Opposition Coalition } & $(0.3794)$ & $(0.4934)$ \\
\hline & $5.2090 * * *$ & $6.1896^{* * *}$ \\
\hline \multirow[t]{2}{*}{ CAR $*$ Opp. Coalition } & $(0.5897)$ & $(0.7412)$ \\
\hline & $-4.6774 * * *$ & $-4.8516^{* * *}$ \\
\hline \multirow[t]{2}{*}{ International Conditionality } & $(0.4987)$ & $(0.4895)$ \\
\hline & $4.7569^{* * *}$ & $5.1871 * * *$ \\
\hline \multirow[t]{2}{*}{ CAR $*$ Conditionality } & $(0.8914)$ & $(1.0729)$ \\
\hline & $1.1386^{* * *}$ & $1.5887^{* * *}$ \\
\hline \multirow[t]{2}{*}{ Main Election } & $(0.4152)$ & $(0.3985)$ \\
\hline & $-0.7959 * *$ & $-1.0300 * *$ \\
\hline \multirow[t]{2}{*}{ Incumbent running } & $(0.3727)$ & $(0.4269)$ \\
\hline & -0.0229 & $-0.0706^{*}$ \\
\hline \multirow[t]{2}{*}{ \# Previous elections } & $(0.0402)$ & $(0.0400)$ \\
\hline & $-0.3085^{*}$ & -0.2063 \\
\hline \multirow[t]{2}{*}{ GDP per capita (lagged 1 year) } & $(0.1776)$ & $(0.1761)$ \\
\hline & -0.0064 & -0.0033 \\
\hline \multirow[t]{2}{*}{ GDP growth (lagged 1 year) } & $(0.0257)$ & $(0.0252)$ \\
\hline & 0.0000 & 0.0000 \\
\hline \multirow[t]{2}{*}{ Central and Eastern Europe } & (.) & (.) \\
\hline & $1.5457^{* * *}$ & $2.0771 * * *$ \\
\hline \multirow[t]{2}{*}{ Americas } & $(0.5179)$ & $(0.6254)$ \\
\hline & & 0.0000 \\
\hline \multirow[t]{2}{*}{ Alternation, Previous Elec. } & & (.) \\
\hline & & $-0.6057 * *$ \\
\hline \multirow[t]{2}{*}{ Misconduct Intensity } & & $(0.2418)$ \\
\hline & & 0.0110 \\
\hline \multirow[t]{2}{*}{ Prior Liberalization } & & $(0.1312)$ \\
\hline & & $-0.4856 *$ \\
\hline Regime Openness & & $(0.2618)$ \\
\hline \multirow[t]{2}{*}{ Constant } & 0.3311 & 1.7548 \\
\hline & $(1.1729)$ & $(1.5803)$ \\
\hline $\mathrm{N}$ & 168 & 165 \\
\hline Pseudo-R2 & 0.281 & 0.371 \\
\hline Log Likelihood & -32.907 & -28.641 \\
\hline
\end{tabular}




\begin{tabular}{|c|cr|}
\hline \multicolumn{3}{|c|}{ Polity IV: HYB; thresholds: 6 \& -6} \\
\hline & Model 3 & Model 5 \\
\hline
\end{tabular}

gdpgrowth_lag1 $>6.7$

predicts data perfectly gdpgrowth_lag1 > 6.7 predicts data perfectly

\begin{tabular}{|c|cr|}
\hline \multicolumn{2}{|c|}{ Polity IV: HYB; thresholds: 8 \& 1 } \\
\hline & Model 3 & Model 5 \\
\hline
\end{tabular}

bm_incrun_fill $>0$ predicts data perfectly bm_incrun_fill $>0$ predicts data perfectly

\begin{tabular}{|c|c|c|}
\hline \multicolumn{3}{|c|}{ Przeworski et al. 2000} \\
\hline & Mode1 3 & Mode1 5 \\
\hline \multirow{3}{*}{$\begin{array}{l}\text { Competitive Authoritarian } \\
\text { Regime (CAR) }\end{array}$} & -0.3919 & convergence not \\
\hline & $(0.4759)$ & achieved \\
\hline & $-4.1547 * * *$ & \\
\hline \multirow[t]{2}{*}{ Opposition Coalition } & $(0.3827)$ & \\
\hline & $4.5298 * * *$ & \\
\hline CAR * Opp. Coalition & $(0.9083)$ & \\
\hline International & 0.8757 & \\
\hline \multirow[t]{2}{*}{ Conditionality } & $(1.2394)$ & \\
\hline & 0.0218 & \\
\hline \multirow[t]{2}{*}{ CAR $*$ Conditionality } & $(1.4795)$ & \\
\hline & 0.0000 & \\
\hline \multirow[t]{2}{*}{ Main Election } & $()$. & \\
\hline & $-0.7156^{*}$ & \\
\hline \multirow[t]{2}{*}{ Incumbent running } & $(0.4283)$ & \\
\hline & $0.1091 * *$ & \\
\hline \# Previous elections & $(0.0444)$ & \\
\hline \multirow{2}{*}{$\begin{array}{l}\text { GDP per capita (lagged } 1 \\
\text { year) }\end{array}$} & $-0.5540^{*}$ & \\
\hline & $(0.2830)$ & \\
\hline \multirow{2}{*}{$\begin{array}{l}\text { GDP growth (lagged } 1 \\
\text { year) }\end{array}$} & -0.0156 & \\
\hline & $(0.0446)$ & \\
\hline Central and Eastern & 0.0000 & \\
\hline & $()$. & \\
\hline \multirow{2}{*}{ Americas } & 1.1935 & \\
\hline & $(0.7486)$ & \\
\hline \multirow[t]{2}{*}{ Constant } & 2.0889 & \\
\hline & $(1.9708)$ & \\
\hline $\mathrm{N}$ & 66 & \\
\hline Pseudo-R2 & 0.221 & \\
\hline Log Likelihood & -20.486 & \\
\hline \multicolumn{2}{|c|}{$* \mathrm{p}<0.10 ; * * \mathrm{p}<0.05 ; * * * \mathrm{p}<0.01$} & \\
\hline
\end{tabular}




\begin{tabular}{|c|c|c|}
\hline \multicolumn{3}{|c|}{ Reich 2002} \\
\hline & Model 3 & Model 5 \\
\hline $\begin{array}{l}\text { Competitive Authoritarian Regime } \\
\text { (CAR) }\end{array}$ & $\begin{array}{c}-0.3792 \\
(0.4845) \\
-4.2329 * * *\end{array}$ & $\begin{array}{c}-0.3047 \\
(0.5460) \\
-3.6964 * * *\end{array}$ \\
\hline Opposition Coalition & $\begin{array}{c}(0.5704) \\
4.3979 * * *\end{array}$ & $\begin{array}{c}(0.7781) \\
3.9795^{* * *}\end{array}$ \\
\hline CAR $*$ Opp. Coalition & $\begin{array}{c}(0.9271) \\
-4.9293^{* * *}\end{array}$ & $\begin{array}{c}(1.0140) \\
1.6078\end{array}$ \\
\hline International Conditionality & $\begin{array}{c}(0.6888) \\
6.2633^{* * *}\end{array}$ & $\begin{array}{c}(0.9992) \\
0.0000\end{array}$ \\
\hline CAR $*$ Conditionality & $\begin{array}{l}(1.2283) \\
1.0218^{*}\end{array}$ & $\begin{array}{c}(.) \\
1.6159 * *\end{array}$ \\
\hline Main Election & $\begin{array}{l}(0.5459) \\
-0.0713\end{array}$ & $\begin{array}{c}(0.7962) \\
0.0010\end{array}$ \\
\hline Incumbent running & $\begin{array}{c}(0.6348) \\
0.0406\end{array}$ & $\begin{array}{c}(0.5603) \\
0.0175\end{array}$ \\
\hline \# Previous elections & $\begin{array}{c}(0.0626) \\
-0.7431 * *\end{array}$ & $\begin{array}{c}(0.0651) \\
-0.7546^{*}\end{array}$ \\
\hline GDP per capita (lagged 1 year) & $\begin{array}{l}(0.3703) \\
-0.0255\end{array}$ & $\begin{array}{l}(0.4544) \\
-0.0358\end{array}$ \\
\hline GDP growth (lagged 1 year) & $\begin{array}{c}(0.0316) \\
0.0000\end{array}$ & $\begin{array}{c}(0.0296) \\
0.0000\end{array}$ \\
\hline Central and Eastern Europe & $\begin{array}{c}(.) \\
2.0665^{* *}\end{array}$ & $\begin{array}{c}(.) \\
2.6546^{* * *}\end{array}$ \\
\hline Americas & $(0.8617)$ & $\begin{array}{c}(0.9480) \\
0.0000\end{array}$ \\
\hline Alternation, Previous Elec. & & $\begin{array}{c}(.) \\
-0.7699\end{array}$ \\
\hline Misconduct Intensity & & $\begin{array}{c}(0.4905) \\
0.0211\end{array}$ \\
\hline Prior Liberalization & & $\begin{array}{l}(0.2469) \\
-0.3381\end{array}$ \\
\hline Regime Openness & & $(0.3053)$ \\
\hline Constant & $\begin{array}{l}2.4620 \\
(2.3452)\end{array}$ & $\begin{array}{c}3.8715 \\
(2.5028)\end{array}$ \\
\hline $\mathrm{N}$ & 74 & 71 \\
\hline Pseudo-R2 & 0.284 & 0.374 \\
\hline Log Likelihood & -18.138 & -15.643 \\
\hline
\end{tabular}

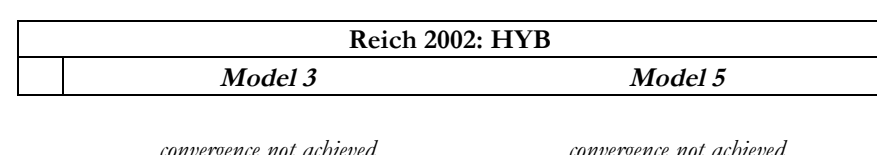

convergence not acbieved

convergence not achieved

\begin{tabular}{|c|c|c|}
\hline \multicolumn{3}{|c|}{ Svolik 2012} \\
\hline & Mode1 3 & Model 5 \\
\hline \multirow{3}{*}{$\begin{array}{l}\text { Competitive Authoritarian Regime } \\
\text { (CAR) }\end{array}$} & 0.0072 & -0.0884 \\
\hline & $(0.4830)$ & $(0.6107)$ \\
\hline & $-3.9916^{* * *}$ & $-4.5643^{* * *}$ \\
\hline \multirow[t]{2}{*}{ Opposition Coalition } & $(0.4157)$ & $(0.5320)$ \\
\hline & $3.8106^{* * *}$ & $5.1688^{* * *}$ \\
\hline \multirow[t]{2}{*}{ CAR $*$ Opp. Coalition } & $(0.7655)$ & $(0.9728)$ \\
\hline & $-5.1135^{* * *}$ & $-9.6924 * * *$ \\
\hline \multirow[t]{2}{*}{ International Conditionality } & $(0.6064)$ & (1.3058) \\
\hline & $5.3903^{* * *}$ & $10.6809^{* * *}$ \\
\hline \multirow[t]{2}{*}{ CAR $*$ Conditionality } & $(1.0303)$ & $(1.5577)$ \\
\hline & 0.0000 & 0.0000 \\
\hline \multirow[t]{2}{*}{ Main Election } & $()$. & (.) \\
\hline & $-1.4072^{* * *}$ & $-1.9563^{* * *}$ \\
\hline \multirow[t]{2}{*}{ Incumbent running } & $(0.4108)$ & $(0.6597)$ \\
\hline & 0.0195 & $-0.1200^{*}$ \\
\hline \multirow[t]{2}{*}{ \# Previous elections } & $(0.0413)$ & $(0.0675)$ \\
\hline & $-0.6795^{* *}$ & $-0.5775^{*}$ \\
\hline \multirow[t]{2}{*}{ GDP per capita (lagged 1 year) } & $(0.3307)$ & $(0.3476)$ \\
\hline & -0.0312 & 0.0205 \\
\hline \multirow[t]{2}{*}{ GDP growth (lagged 1 year) } & $(0.0361)$ & $(0.0393)$ \\
\hline & 0.0000 & 0.0000 \\
\hline \multirow[t]{2}{*}{ Central and Eastern Europe } & $()$. & (.) \\
\hline & $2.4353^{* * *}$ & $3.8184^{* * *}$ \\
\hline \multirow[t]{2}{*}{ Americas } & $(0.7465)$ & $(1.0283)$ \\
\hline & & $5.8354 * * *$ \\
\hline \multirow[t]{2}{*}{ Alternation, Previous Elec. } & & $(0.5918)$ \\
\hline & & $-1.6414^{* * *}$ \\
\hline \multirow[t]{2}{*}{ Misconduct Intensity } & & $(0.5640)$ \\
\hline & & $0.5385^{*}$ \\
\hline \multirow[t]{2}{*}{ Prior Liberalization } & & $(0.3011)$ \\
\hline & & -0.3907 \\
\hline \multirow{3}{*}{$\begin{array}{l}\text { Regime Openness } \\
\text { Constant }\end{array}$} & & $(0.2660)$ \\
\hline & $3.6620^{*}$ & $6.4152^{* *}$ \\
\hline & $(2.2029)$ & $(2.5311)$ \\
\hline $\mathrm{N}$ & 91 & 91 \\
\hline Pseudo-R2 & 0.356 & 0.551 \\
\hline Log Likelihood & -22.849 & -15.934 \\
\hline
\end{tabular}




\begin{tabular}{|c|c|c|}
\hline \multicolumn{3}{|c|}{ Ulfelder 2012} \\
\hline & Model 3 & Model 5 \\
\hline \multirow{3}{*}{$\begin{array}{l}\text { Competitive Authoritarian Regime } \\
\text { (CAR) }\end{array}$} & -0.0691 & -0.1284 \\
\hline & $(0.3687)$ & $(0.3502)$ \\
\hline & $-4.6724^{* * *}$ & $-4.6314^{* * *}$ \\
\hline \multirow[t]{2}{*}{ Opposition Coalition } & $(0.3692)$ & $(0.4876)$ \\
\hline & $4.7194^{* * *}$ & $4.9534 * * *$ \\
\hline \multirow[t]{2}{*}{ CAR * Opp. Coalition } & $(0.7502)$ & $(0.7699)$ \\
\hline & $-5.3721 * * *$ & $-6.7157 * * *$ \\
\hline \multirow[t]{2}{*}{ International Conditionality } & $(0.4812)$ & (1.0108) \\
\hline & $5.3474^{* * *}$ & $6.8603 * * *$ \\
\hline \multirow[t]{2}{*}{ CAR $*$ Conditionality } & $(0.8697)$ & $(1.2130)$ \\
\hline & $1.9384^{* * *}$ & $2.1973^{* *}$ \\
\hline \multirow[t]{2}{*}{ Main Election } & $(0.6290)$ & $(0.8660)$ \\
\hline & $-1.2494 * * *$ & $-1.1915^{* *}$ \\
\hline \multirow[t]{2}{*}{ Incumbent running } & $(0.4049)$ & $(0.4654)$ \\
\hline & -0.0002 & -0.0399 \\
\hline \multirow[t]{2}{*}{ \# Previous elections } & $(0.0272)$ & $(0.0324)$ \\
\hline & $-0.4435 * *$ & $-0.3824 * *$ \\
\hline \multirow[t]{2}{*}{ GDP per capita (lagged 1 year) } & $(0.2026)$ & $(0.1762)$ \\
\hline & 0.0152 & 0.0510 \\
\hline \multirow[t]{2}{*}{ GDP growth (lagged 1 year) } & $(0.0354)$ & $(0.0392)$ \\
\hline & 0.0000 & 0.0000 \\
\hline \multirow[t]{2}{*}{ Central and Eastern Europe } & $()$. & $()$. \\
\hline & $1.8763^{* * *}$ & $2.0342^{* * *}$ \\
\hline \multirow[t]{2}{*}{ Americas } & $(0.5092)$ & $(0.5847)$ \\
\hline & & $1.9139 * * *$ \\
\hline \multirow[t]{2}{*}{ Alternation, Previous Elec. } & & $(0.6620)$ \\
\hline & & $-0.7169 * *$ \\
\hline \multirow[t]{2}{*}{ Misconduct Intensity } & & $(0.3131)$ \\
\hline & & 0.0643 \\
\hline \multirow[t]{2}{*}{ Prior Liberalization } & & $(0.1567)$ \\
\hline & & 0.1039 \\
\hline Regime Openness & & $(0.2403)$ \\
\hline \multirow[t]{2}{*}{ Constant } & 0.3989 & 0.1559 \\
\hline & $(1.4169)$ & $(1.7168)$ \\
\hline $\mathrm{N}$ & 173 & 173 \\
\hline Pseudo-R2 & 0.365 & 0.448 \\
\hline Log Likelihood & -32.415 & -28.167 \\
\hline
\end{tabular}

\begin{tabular}{|c|c|c|}
\hline \multicolumn{3}{|c|}{ Wahman et al. 2013} \\
\hline & Model 3 & Model 5 \\
\hline $\begin{array}{l}\text { Competitive Authoritarian Regime } \\
\text { (CAR) }\end{array}$ & $\begin{array}{c}0.0856 \\
(0.4985) \\
-3.5641 * * *\end{array}$ & $\begin{array}{c}0.0081 \\
(0.6806) \\
-2.8459 * * *\end{array}$ \\
\hline Opposition Coalition & $\begin{array}{c}(0.3593) \\
4.0089 * * *\end{array}$ & $\begin{array}{c}(0.7823) \\
3.5964 * * *\end{array}$ \\
\hline CAR * Opp. Coalition & $\begin{array}{c}(0.8048) \\
-3.7319 * * *\end{array}$ & $\begin{array}{c}(1.1469) \\
-3.5255^{* * *}\end{array}$ \\
\hline International Conditionality & $\begin{array}{c}(0.6143) \\
4.0905^{* * *}\end{array}$ & $\begin{array}{c}(0.6236) \\
4.9939 * * *\end{array}$ \\
\hline CAR * Conditionality & $\begin{array}{c}(1.0243) \\
0.0000\end{array}$ & $\begin{array}{c}(1.1552) \\
0.0000\end{array}$ \\
\hline Main Election & $\begin{array}{c}(.) \\
0.0280\end{array}$ & $\begin{array}{c}(.) \\
0.0361\end{array}$ \\
\hline Incumbent running & $\begin{array}{l}(0.4826) \\
0.0550^{* *}\end{array}$ & $\begin{array}{l}(0.5974) \\
-0.0324\end{array}$ \\
\hline \# Previous elections & $\begin{array}{l}(0.0273) \\
-0.4058\end{array}$ & $\begin{array}{l}(0.0539) \\
-0.5021\end{array}$ \\
\hline GDP per capita (lagged 1 year) & $\begin{array}{l}(0.2750) \\
-0.0315\end{array}$ & $\begin{array}{l}(0.3245) \\
-0.0014\end{array}$ \\
\hline GDP growth (lagged 1 year) & $\begin{array}{c}(0.0388) \\
0.0000\end{array}$ & $\begin{array}{c}(0.0567) \\
0.0000\end{array}$ \\
\hline Central and Eastern Europe & $\begin{array}{c}(.) \\
2.2690^{* * *}\end{array}$ & $\begin{array}{c}(.) \\
3.5085^{* * *}\end{array}$ \\
\hline Americas & $(0.6077)$ & $\begin{array}{c}(0.6914) \\
0.0000\end{array}$ \\
\hline Alternation, Previous Elec. & & $\begin{array}{c}(.) \\
-1.2308^{* *}\end{array}$ \\
\hline Misconduct Intensity & & $\begin{array}{c}(0.5270) \\
0.5246\end{array}$ \\
\hline Prior Liberalization & & $\begin{array}{c}(0.3220) \\
0.0070\end{array}$ \\
\hline Regime Openness & & $(0.2297)$ \\
\hline Constant & $\begin{array}{c}0.5328 \\
(1.8768)\end{array}$ & $\begin{array}{c}2.3752 \\
(2.3105)\end{array}$ \\
\hline $\mathrm{N}$ & 92 & 90 \\
\hline Pseudo-R2 & 0.280 & 0.432 \\
\hline Log Likelihood & -21.220 & -16.617 \\
\hline
\end{tabular}


Appendix VI: Robustness Checks: Andersen et al. 2014

\begin{tabular}{|c|c|c|}
\hline \multicolumn{3}{|c|}{ Bernhard et al. 2001} \\
\hline & Model 3 & Model 6 \\
\hline $\begin{array}{l}\text { Bureaucratic } \\
\text { quality }\end{array}$ & $\begin{array}{l}-1.7793^{*} \\
(1.0605)\end{array}$ & $\begin{array}{l}-1.9088 \\
(1.2839)\end{array}$ \\
\hline Growth in & $9.6982 * *$ & $9.7522 *$ \\
\hline GDP/cap. & $(4.7661)$ & $(5.1197)$ \\
\hline Oil value/cap. & 0.0540 & 0.1011 \\
\hline \multirow[t]{2}{*}{$(\log$} & $(0.0442)$ & $(0.0837)$ \\
\hline & $1.7265^{*}$ & 1.6740 \\
\hline \multirow{2}{*}{ Armed conflict } & $(1.0268)$ & $(1.1392)$ \\
\hline & 0.0000 & 0.0000 \\
\hline International war & $()$. & $()$. \\
\hline $\begin{array}{l}\text { Mixed (versus } \\
\text { parliamentary) }\end{array}$ & 0.5665 & 0.4377 \\
\hline regime & $(0.8785)$ & $(0.9959)$ \\
\hline $\begin{array}{l}\text { Presidential } \\
\text { (versus } \\
\text { parliamentary) }\end{array}$ & -0.4219 & -0.2654 \\
\hline \multirow[t]{2}{*}{ regime } & $(0.8469)$ & $(0.9736)$ \\
\hline & -16.2544 & -20.6232 \\
\hline \multirow[t]{2}{*}{ time } & $(16.1565)$ & $(18.3082)$ \\
\hline & 0.3567 & 0.4452 \\
\hline \multirow[t]{2}{*}{ time2 } & $(0.3253)$ & $(0.3721)$ \\
\hline & -0.0025 & -0.0031 \\
\hline \multirow[t]{2}{*}{ time3 } & $(0.0022)$ & $(0.0025)$ \\
\hline & 0.0234 & 0.0398 \\
\hline \multirow[t]{2}{*}{ spellcount } & $(0.0215)$ & $(0.0349)$ \\
\hline & & -0.8596 \\
\hline GDP/cap. (log) & & $(0.9255)$ \\
\hline \multirow[t]{2}{*}{ Constant } & 238.1597 & 315.4434 \\
\hline & $(266.3380)$ & $(300.8187)$ \\
\hline $\mathrm{N}$ & 1080 & 1080 \\
\hline \multicolumn{3}{|c|}{$* \mathrm{p}<0.10, * * \mathrm{p}<0.05, * * * \mathrm{p}<0.01$} \\
\hline
\end{tabular}

\begin{tabular}{|l|cc|}
\hline \multicolumn{3}{|c|}{ Boix et al. 2013 } \\
\hline Bureaucratic & $-0.4558^{* * *}$ & $-0.3234^{*}$ \\
quality & $(0.1707)$ & $(0.1892)$ \\
Growth in & -0.6132 & -0.5237 \\
GDP/cap. & $(2.6824)$ & $(2.7486)$ \\
Oil value/cap. & 0.0315 & $0.0451^{*}$ \\
(log & $(0.0199)$ & $(0.0241)$ \\
& 0.2671 & 0.1835 \\
Armed conflict & $(0.3154)$ & $(0.3237)$ \\
& 0.2323 & -0.0738 \\
International war & $(0.5225)$ & $(0.5554)$ \\
Mixed (versus & $-0.7971 *$ & $-0.8158^{*}$ \\
parliamentary) & & \\
regime & $(0.4614)$ & $(0.4822)$ \\
Presidential & $-0.6608 *$ & $-0.6215^{*}$ \\
(versus & & \\
parliamentary) & & \\
regime & $(0.3384)$ & $(0.3410)$ \\
& 6.7968 & 6.6381 \\
time & $(6.1111)$ & $(6.0521)$ \\
time2 & -0.1307 & -0.1287 \\
time3 & $(0.1176)$ & $(0.1168)$ \\
spellcount & 0.0008 & 0.0008 \\
GDP/cap. (log) & $(0.0007)$ & $(0.0007)$ \\
Constant & -0.0086 & -0.0048 \\
N & $(0.0096)$ & $(0.0098)$ \\
\hline \multicolumn{1}{|c}{$* 10, * *$} & \\
& & -0.3206 \\
& -118.3003 & -112.2736 \\
& $(105.1184)$ & $(103.8744)$ \\
& 1296 & 1296 \\
\hline
\end{tabular}

\begin{tabular}{|c|c|c|}
\hline \multicolumn{3}{|c|}{ Cheibub et al. 2010} \\
\hline & Model 3 & Model 6 \\
\hline $\begin{array}{l}\text { Bureaucratic } \\
\text { quality }\end{array}$ & $\begin{array}{l}-0.1922 \\
(0.1727)\end{array}$ & $\begin{array}{c}0.0259 \\
(0.2082)\end{array}$ \\
\hline Growth in & $-4.8721 * *$ & $-4.7466 * *$ \\
\hline GDP/cap. & $(2.1115)$ & $(2.1598)$ \\
\hline Oil value/cap. & 0.0104 & 0.0300 \\
\hline \multirow[t]{2}{*}{$(\log$} & $(0.0176)$ & $(0.0224)$ \\
\hline & $0.5300^{*}$ & 0.4738 \\
\hline \multirow[t]{2}{*}{ Armed conflict } & $(0.3046)$ & $(0.3144)$ \\
\hline & 0.3821 & 0.0085 \\
\hline International war & $(0.5463)$ & $(0.5826)$ \\
\hline $\begin{array}{l}\text { Mixed (versus } \\
\text { parliamentary) }\end{array}$ & -0.3561 & -0.3688 \\
\hline regime & $(0.3764)$ & $(0.3955)$ \\
\hline $\begin{array}{l}\text { Presidential } \\
\text { (versus } \\
\text { parliamentary) }\end{array}$ & $-0.7106^{* *}$ & $-0.6910^{*}$ \\
\hline \multirow[t]{2}{*}{ regime } & $(0.3543)$ & $(0.3635)$ \\
\hline & 1.7206 & 1.7499 \\
\hline \multirow[t]{2}{*}{ time } & $(5.1821)$ & $(5.2136)$ \\
\hline & -0.0314 & -0.0334 \\
\hline \multirow[t]{2}{*}{ time2 } & $(0.0993)$ & $(0.1002)$ \\
\hline & 0.0002 & 0.0002 \\
\hline \multirow[t]{2}{*}{ time3 } & $(0.0006)$ & $(0.0006)$ \\
\hline & -0.0298 & -0.0253 \\
\hline \multirow[t]{2}{*}{ spellcount } & $(0.0183)$ & $(0.0171)$ \\
\hline & & $-0.4356^{* *}$ \\
\hline GDP/cap. (log) & & $(0.2151)$ \\
\hline \multirow[t]{2}{*}{ Constant } & -32.8555 & -28.8868 \\
\hline & $(89.5133)$ & $(89.7624)$ \\
\hline $\mathrm{N}$ & 1396 & 1396 \\
\hline
\end{tabular}




\begin{tabular}{|l|cc|}
\hline \multicolumn{3}{|c|}{ FH: free, FIW } \\
\hline & Model 3 & Model 6 \\
\hline Bureaucratic & $-0.3030^{* *}$ & -0.0611 \\
quality & $(0.1230)$ & $(0.1466)$ \\
Growth in & -2.5050 & -2.2057 \\
GDP/cap. & $(2.1024)$ & $(2.1742)$ \\
Oil value/cap. & $0.0306 * *$ & $0.0436 * * *$ \\
(log & $(0.0130)$ & $(0.0158)$ \\
Armed & $0.6350^{* *}$ & 0.2820 \\
conflict & $(0.2901)$ & $(0.3238)$ \\
International & 0.3516 & -0.0971 \\
war & $(0.4726)$ & $(0.5126)$ \\
Mixed (versus & -0.2742 & -0.4547 \\
parliamentary) & & \\
regime & $(0.3896)$ & $(0.4409)$ \\
Presidential & 0.3506 & 0.2033 \\
(versus & & \\
parliamentary) & & \\
regime & $(0.2852)$ & $(0.3131)$ \\
& $11.0822^{* * *}$ & $12.6299 * * *$ \\
time & $(3.8111)$ & $(4.0910)$ \\
& $-0.2035^{* * *}$ & $-0.2343^{* * *}$ \\
time2 & $(0.0709)$ & $(0.0761)$ \\
time3 & $0.0012^{* * *}$ & $0.0014 * * *$ \\
spellcount & $(0.0004)$ & $(0.0005)$ \\
GDP/cap. & $-0.0117 *$ & -0.0057 \\
log) & $(0.0063)$ & $(0.0064)$ \\
Constant & & $-0.5735 * * *$ \\
N & $-200.2292^{* * *}$ & $-221.3576 * * *$ \\
\hline & $(67.8404)$ & $(72.5656)$ \\
& 1173 & 1173 \\
\hline
\end{tabular}

\begin{tabular}{|c|c|c|}
\hline \multicolumn{3}{|c|}{ FH: partly free, FIW } \\
\hline & Model 3 & Model 6 \\
\hline $\begin{array}{l}\text { Bureaucratic } \\
\text { quality }\end{array}$ & $\begin{array}{c}-0.4575^{*} \\
(0.2635)\end{array}$ & $\begin{array}{l}-0.4246 \\
(0.3035)\end{array}$ \\
\hline Growth in & -4.1742 & -4.1150 \\
\hline GDP/cap. & $(2.6366)$ & $(2.6568)$ \\
\hline Oil value/cap. & -0.0044 & -0.0014 \\
\hline \multirow[t]{2}{*}{$(\log$} & $(0.0236)$ & $(0.0274)$ \\
\hline & 0.5189 & 0.5099 \\
\hline \multirow[t]{2}{*}{ Armed conflict } & $(0.4054)$ & $(0.4068)$ \\
\hline & 0.8295 & 0.7664 \\
\hline International war & $(0.6444)$ & $(0.7040)$ \\
\hline $\begin{array}{l}\text { Mixed (versus } \\
\text { parliamentary) }\end{array}$ & -0.3401 & -0.3418 \\
\hline regime & $(0.4840)$ & $(0.4834)$ \\
\hline $\begin{array}{l}\text { Presidential } \\
\text { (versus } \\
\text { parliamentary) }\end{array}$ & $-1.1872^{*}$ & $-1.1753^{*}$ \\
\hline \multirow[t]{2}{*}{ regime } & $(0.6223)$ & $(0.6248)$ \\
\hline & -0.8635 & -0.8466 \\
\hline \multirow[t]{2}{*}{ time } & $(6.4613)$ & $(6.4116)$ \\
\hline & 0.0214 & 0.0208 \\
\hline \multirow[t]{2}{*}{ time2 } & $(0.1224)$ & $(0.1216)$ \\
\hline & -0.0002 & -0.0002 \\
\hline \multirow[t]{2}{*}{ time3 } & $(0.0008)$ & $(0.0008)$ \\
\hline & -0.0309 & -0.0291 \\
\hline \multirow[t]{2}{*}{ spellcount } & $(0.0340)$ & $(0.0330)$ \\
\hline & & -0.0660 \\
\hline GDP/cap. (log) & & $(0.2999)$ \\
\hline \multirow[t]{2}{*}{ Constant } & 9.0718 & 9.5586 \\
\hline & $(112.8492)$ & (111.9404) \\
\hline $\mathrm{N}$ & 1583 & 1583 \\
\hline \multicolumn{3}{|c|}{$* \mathrm{p}<0.10, * * \mathrm{p}<0.05, * * * \mathrm{p}<0.01$} \\
\hline
\end{tabular}

\begin{tabular}{|c|c|c|}
\hline \multicolumn{3}{|c|}{ FH: free, PR } \\
\hline & Model 3 & Model 6 \\
\hline $\begin{array}{l}\text { Bureaucratic } \\
\text { quality }\end{array}$ & $\begin{array}{c}-0.3212^{* * *} \\
(0.1079)\end{array}$ & $\begin{array}{l}-0.0659 \\
(0.1301)\end{array}$ \\
\hline Growth in & -0.2517 & 0.4781 \\
\hline GDP/cap. & $(1.9633)$ & $(2.0489)$ \\
\hline \multirow{3}{*}{$\begin{array}{l}\text { Oil value/cap. } \\
\text { (log }\end{array}$} & $0.0219 * *$ & $0.0299 * *$ \\
\hline & $(0.0109)$ & $(0.0130)$ \\
\hline & $0.5733^{* *}$ & 0.2996 \\
\hline Armed conflict & $(0.2442)$ & $(0.2620)$ \\
\hline International & -0.1661 & -0.7046 \\
\hline war & $(0.5213)$ & $(0.5800)$ \\
\hline $\begin{array}{l}\text { Mixed (versus } \\
\text { parliamentary) }\end{array}$ & -0.4648 & -0.5617 \\
\hline regime & $(0.3151)$ & $(0.3522)$ \\
\hline $\begin{array}{l}\text { Presidential } \\
\text { (versus } \\
\text { parliamentary) }\end{array}$ & 0.1898 & 0.1782 \\
\hline \multirow[t]{2}{*}{ regime } & $(0.2215)$ & $(0.2386)$ \\
\hline & $5.8306^{*}$ & $5.8321 *$ \\
\hline \multirow[t]{2}{*}{ time } & $(3.1742)$ & (3.1764) \\
\hline & $-0.1020^{*}$ & $-0.1039 *$ \\
\hline \multirow[t]{2}{*}{ time2 } & $(0.0595)$ & $(0.0597)$ \\
\hline & 0.0006 & 0.0006 \\
\hline \multirow[t]{2}{*}{ time3 } & $(0.0004)$ & $(0.0004)$ \\
\hline & $-0.0118^{* *}$ & -0.0055 \\
\hline \multirow[t]{2}{*}{ spellcount } & $(0.0056)$ & $(0.0055)$ \\
\hline & & $-0.5105^{* * *}$ \\
\hline GDP/cap. (log) & & $(0.1546)$ \\
\hline \multirow[t]{2}{*}{ Constant } & $-110.4410^{* *}$ & $-104.8760^{*}$ \\
\hline & $(56.0490)$ & $(55.9456)$ \\
\hline $\mathrm{N}$ & 1199 & 1199 \\
\hline \multicolumn{3}{|c|}{$* \mathrm{p}<0.10, * * \mathrm{p}<0.05, * * * \mathrm{p}<0.01$} \\
\hline
\end{tabular}




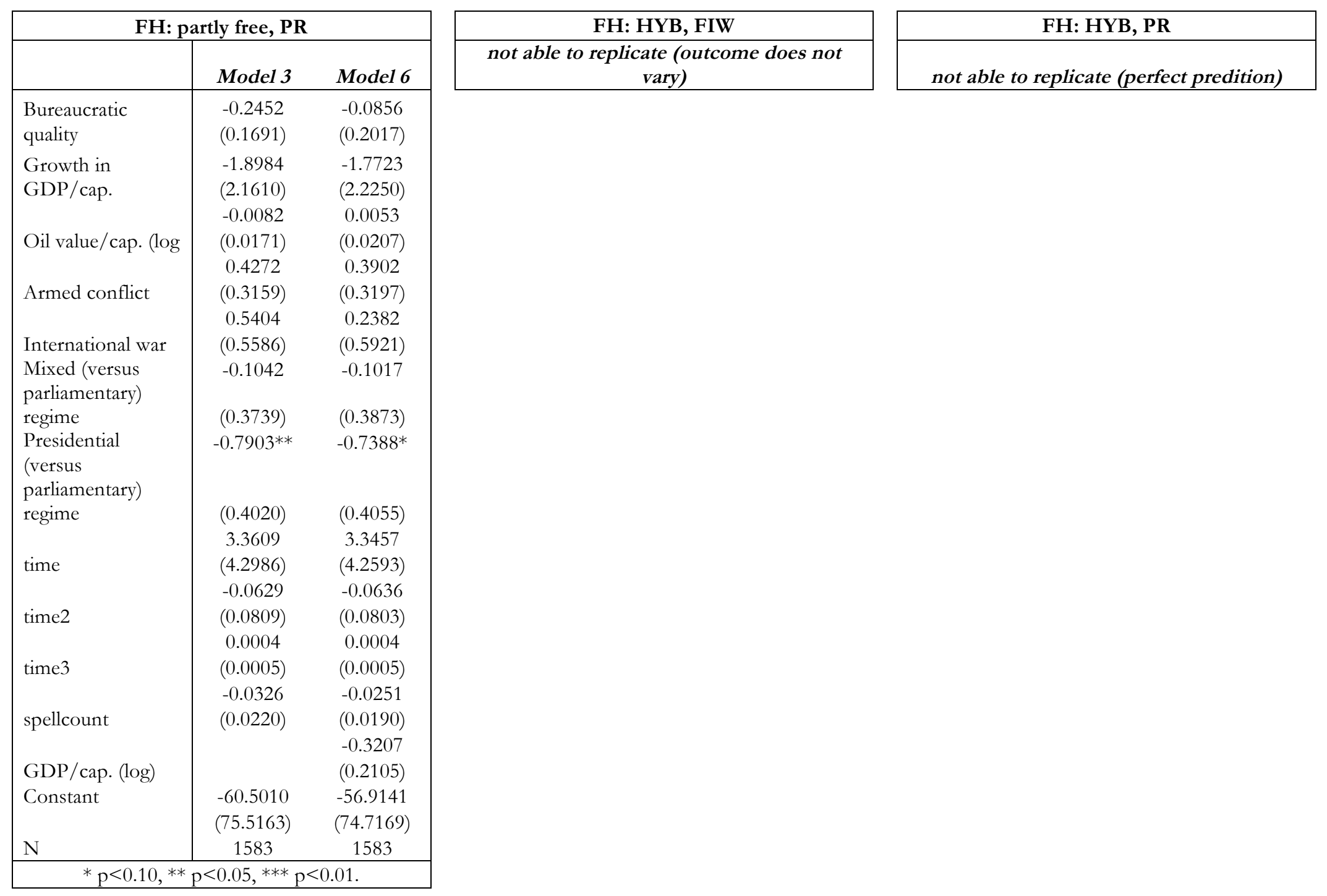




\begin{tabular}{|l|l|}
\hline \multicolumn{2}{|c|}{ Gasiorowski 1996} \\
\hline not able to replicate (not convave) & \\
\hline
\end{tabular}

\begin{tabular}{|c|}
\hline Gasiorowski 1996: HYB \\
\hline $\begin{array}{c}\text { not able to replicate (outcome does not } \\
\text { vary) }\end{array}$ \\
\hline
\end{tabular}

\begin{tabular}{|c|c|c|}
\hline \multicolumn{3}{|c|}{ Gates et al. 2006} \\
\hline & Model 3 & Model 6 \\
\hline $\begin{array}{l}\text { Bureaucratic } \\
\text { quality }\end{array}$ & $\begin{array}{c}-0.4788^{* * *} \\
(0.1828)\end{array}$ & $\begin{array}{c}-0.3485^{*} \\
(0.2109)\end{array}$ \\
\hline Growth in & 2.9046 & 3.0681 \\
\hline GDP/cap. & $(2.2614)$ & $(2.2917)$ \\
\hline Oil value/cap. & -0.0100 & 0.0003 \\
\hline \multirow[t]{2}{*}{$(\log$} & $(0.0192)$ & $(0.0209)$ \\
\hline & 0.3249 & 0.2503 \\
\hline \multirow[t]{2}{*}{ Armed conflict } & $(0.3816)$ & $(0.3827)$ \\
\hline & 0.3683 & 0.2236 \\
\hline International war & $(0.5963)$ & $(0.6147)$ \\
\hline $\begin{array}{l}\text { Mixed (versus } \\
\text { parliamentary) }\end{array}$ & -0.3432 & -0.2887 \\
\hline regime & $(0.4479)$ & $(0.4616)$ \\
\hline $\begin{array}{l}\text { Presidential } \\
\text { (versus } \\
\text { parliamentary) }\end{array}$ & -0.6578 & -0.4803 \\
\hline \multirow[t]{2}{*}{ regime } & $(0.4079)$ & $(0.4259)$ \\
\hline & -5.4925 & -3.3017 \\
\hline \multirow[t]{2}{*}{ time } & $(20.9792)$ & $(20.8642)$ \\
\hline & 0.1243 & 0.0766 \\
\hline \multirow[t]{2}{*}{ time2 } & $(0.4317)$ & $(0.4297)$ \\
\hline & -0.0009 & -0.0006 \\
\hline \multirow[t]{2}{*}{ time3 } & $(0.0030)$ & $(0.0029)$ \\
\hline & -0.0512 & -0.0370 \\
\hline \multirow[t]{2}{*}{ spellcount } & $(0.0415)$ & $(0.0368)$ \\
\hline & & -0.3185 \\
\hline GDP/cap. (log) & & $(0.2343)$ \\
\hline \multirow[t]{2}{*}{ Constant } & 77.4029 & 46.6894 \\
\hline & $(338.7462)$ & $(336.4159)$ \\
\hline $\mathrm{N}$ & 797 & 797 \\
\hline
\end{tabular}




\begin{tabular}{|l|cc|}
\hline \multicolumn{3}{|c|}{ Geddes et al. 2014 } \\
\hline & Model 3 & Model 6 \\
\hline Bureaucratic & $-0.4698^{* * *}$ & $-0.3256 *$ \\
quality & $(0.1698)$ & $(0.1901)$ \\
Growth in & 1.6993 & 1.9660 \\
GDP/cap. & $(1.9115)$ & $(1.9533)$ \\
Oil value/cap. & -0.0021 & 0.0122 \\
(log & $(0.0147)$ & $(0.0186)$ \\
& 0.4168 & 0.3298 \\
Armed conflict & $(0.2910)$ & $(0.3041)$ \\
& 0.2729 & -0.0174 \\
International war & $(0.5313)$ & $(0.5627)$ \\
Mixed (versus & -0.1872 & -0.1960 \\
parliamentary) & & \\
regime & $(0.3574)$ & $(0.3895)$ \\
Presidential & $-0.6601 *$ & -0.5937 \\
(versus & & \\
parliamentary) & & \\
regime & $(0.3467)$ & $(0.3683)$ \\
time & 5.3577 & 5.1373 \\
time2 & $(4.7712)$ & $(4.7239)$ \\
time3 & -0.0987 & -0.0957 \\
spellcount & $(0.0895)$ & $(0.0888)$ \\
GDP/cap. (log) & 0.0006 & 0.0006 \\
Constant & $(0.0006)$ & $(0.0006)$ \\
N & -0.0064 & -0.0003 \\
\hline & $(0.0102)$ & $(0.0109)$ \\
& & $-0.3732 *$ \\
& & $(0.2090)$ \\
& -97.2371 & -89.5458 \\
& $(84.2229)$ & $(83.1395)$ \\
& 1480 & 1480 \\
\hline
\end{tabular}

\begin{tabular}{|l|cc|}
\hline \multicolumn{3}{|c|}{ Magaloni et al. 2013 } \\
\hline & Model 3 & Model 6 \\
\hline Bureaucratic & $-0.5174 * *$ & $-0.4214^{*}$ \\
quality & $(0.2479)$ & $(0.2527)$ \\
Growth in & $-5.1574 *$ & $-5.2234 *$ \\
GDP/cap. & $(3.0511)$ & $(3.1121)$ \\
& 0.0030 & 0.0111 \\
Oil value/cap. (log & $(0.0176)$ & $(0.0203)$ \\
& $0.7717 * *$ & $0.7144 * *$ \\
Armed conflict & $(0.3284)$ & $(0.3314)$ \\
& 0.6925 & 0.4836 \\
International war & $(0.5740)$ & $(0.6051)$ \\
Mixed (versus & -0.0789 & -0.1738 \\
parliamentary) & & \\
regime & $(0.4753)$ & $(0.4970)$ \\
Presidential & -0.1668 & -0.1500 \\
(versus & & \\
parliamentary) & & \\
regime & $(0.3922)$ & $(0.3872)$ \\
time & 2.7536 & 2.8092 \\
time2 & $(5.3485)$ & $(5.1412)$ \\
time3 & -0.0487 & -0.0506 \\
spellcount & $(0.0986)$ & $(0.0950)$ \\
GDP/cap. (log) & 0.0003 & 0.0003 \\
Constant & $(0.0006)$ & $(0.0006)$ \\
N & -0.0004 & 0.0023 \\
\hline & $(0.0097)$ & $(0.0103)$ \\
\hline
\end{tabular}

\begin{tabular}{|l|cc|}
\hline \multicolumn{3}{|c|}{ Polity IV: AN=AUT } \\
\hline & Model 3 & Model 6 \\
\hline Bureaucratic & $-0.3485^{* *}$ & -0.2283 \\
quality & $(0.1619)$ & $(0.1749)$ \\
Growth in & 2.4018 & 2.8978 \\
GDP/cap. & $(2.2957)$ & $(2.3738)$ \\
& -0.0206 & -0.0127 \\
Oil value/cap. (log & $(0.0142)$ & $(0.0152)$ \\
& $0.5898^{* *}$ & $0.5155^{*}$ \\
Armed conflict & $(0.2903)$ & $(0.2899)$ \\
& 0.5758 & 0.3833 \\
International war & $(0.5704)$ & $(0.5703)$ \\
Mixed (versus & -0.1177 & -0.1717 \\
parliamentary) & & \\
regime & $(0.4056)$ & $(0.4134)$ \\
Presidential & -0.0224 & 0.0085 \\
(versus & & \\
parliamentary) & & \\
regime & $(0.3330)$ & $(0.3321)$ \\
time & -1.5410 & -1.5466 \\
time2 & $(3.0851)$ & $(3.0489)$ \\
time3 & 0.0289 & 0.0284 \\
spellcount & $(0.0588)$ & $(0.0582)$ \\
GDP/cap. (log) & -0.0002 & -0.0002 \\
Constant & $(0.0004)$ & $(0.0004)$ \\
N & -0.0218 & -0.0162 \\
\hline & $(0.0142)$ & $(0.0135)$ \\
& & $-0.2695^{*}$ \\
& 25.1760 & 27.9510 \\
& $(53.3956)$ & $(52.7161)$ \\
& 1465 & 1465 \\
\hline
\end{tabular}




\begin{tabular}{|l|cc|}
\hline \multicolumn{3}{|c|}{ Polity IV: AN=DEM } \\
\hline & Model 3 & Model 6 \\
\hline Bureaucratic & $-0.4590^{*}$ & -0.2850 \\
quality & $(0.2635)$ & $(0.3212)$ \\
Growth in & -0.1499 & 0.2540 \\
GDP/cap. & $(3.7332)$ & $(4.0757)$ \\
Oil value/cap. & -0.0043 & 0.0201 \\
(log & $(0.0248)$ & $(0.0337)$ \\
& 0.2663 & 0.1282 \\
Armed conflict & $(0.4629)$ & $(0.5036)$ \\
& 0.7911 & 0.4213 \\
International war & $(0.6267)$ & $(0.6625)$ \\
Mixed (versus & -0.2842 & -0.2508 \\
parliamentary) & & \\
regime & $(0.4759)$ & $(0.5046)$ \\
Presidential & 0.0000 & 0.0000 \\
(versus & & \\
parliamentary) & & \\
regime & $()$. & $()$. \\
time & 1.7686 & 1.3382 \\
time2 & $(6.4699)$ & $(6.3755)$ \\
time3 & -0.0316 & -0.0256 \\
spellcount & $(0.1221)$ & $(0.1209)$ \\
GDP/cap. (log) & 0.0002 & 0.0002 \\
Constant & $(0.0008)$ & $(0.0008)$ \\
N & -0.0207 & -0.0165 \\
\hline \multicolumn{1}{|c|}{$*$ p $<0.05, * * * \mathrm{p}<0.01}$. \\
\hline
\end{tabular}

\begin{tabular}{|c|c|c|}
\hline \multicolumn{3}{|c|}{ Polity IV: threshold 7} \\
\hline & Model 3 & Model 6 \\
\hline $\begin{array}{l}\text { Bureaucratic } \\
\text { quality }\end{array}$ & $\begin{array}{c}-0.8829 * * * \\
(0.2600)\end{array}$ & $\begin{array}{c}-0.8080^{* * *} \\
(0.2720)\end{array}$ \\
\hline Growth in & $-6.1385^{* *}$ & $-5.9780 * *$ \\
\hline GDP/cap. & $(2.9086)$ & $(3.0103)$ \\
\hline Oil value/cap. & 0.0265 & $0.0386^{*}$ \\
\hline$(\log$ & $(0.0176)$ & $(0.0221)$ \\
\hline & 0.0554 & -0.0283 \\
\hline Armed conflict & $(0.3629)$ & $(0.3736)$ \\
\hline & 0.5836 & 0.2554 \\
\hline International war & $(0.6153)$ & $(0.6926)$ \\
\hline $\begin{array}{l}\text { Mixed (versus } \\
\text { parliamentary) }\end{array}$ & -0.3344 & -0.5088 \\
\hline regime & $(0.4782)$ & $(0.5274)$ \\
\hline $\begin{array}{l}\text { Presidential } \\
\text { (versus } \\
\text { parliamentary) }\end{array}$ & -0.5874 & -0.6150 \\
\hline regime & $(0.4275)$ & $(0.4422)$ \\
\hline & 2.9849 & 3.3680 \\
\hline time & $(5.0159)$ & $(5.0561)$ \\
\hline & -0.0495 & -0.0570 \\
\hline time2 & $(0.0929)$ & $(0.0937)$ \\
\hline & 0.0003 & 0.0003 \\
\hline time3 & $(0.0006)$ & $(0.0006)$ \\
\hline & -0.0004 & 0.0037 \\
\hline spellcount & $(0.0106)$ & $(0.0114)$ \\
\hline & & -0.3141 \\
\hline GDP/cap. (log) & & $(0.2686)$ \\
\hline Constant & -59.6979 & -63.7101 \\
\hline & (89.5593) & $(90.1260)$ \\
\hline $\mathrm{N}$ & 1345 & 1345 \\
\hline
\end{tabular}

\begin{tabular}{|l|cc|}
\hline \multicolumn{3}{|c|}{ Polity IV: threshold 1 } \\
\hline & Model 3 & Model 6 \\
\hline Bureaucratic & $-0.2999 *$ & -0.0810 \\
quality & $(0.1579)$ & $(0.1880)$ \\
Growth in & -0.1729 & -0.0037 \\
GDP/cap. & $(2.1134)$ & $(2.2112)$ \\
Oil value/cap. & -0.0074 & 0.0131 \\
(log & $(0.0159)$ & $(0.0204)$ \\
& $0.6294 * *$ & $0.5872 * *$ \\
Armed conflict & $(0.2848)$ & $(0.2971)$ \\
& 0.3062 & -0.0806 \\
International war & $(0.5427)$ & $(0.5685)$ \\
Mixed (versus & -0.2402 & -0.2468 \\
parliamentary) & & \\
regime & $(0.3393)$ & $(0.3628)$ \\
Presidential & $-0.9965 * * *$ & $-0.9278 * *$ \\
(versus & & \\
parliamentary) & & \\
regime & $(0.3765)$ & $(0.3832)$ \\
time & 4.8885 & 4.8368 \\
time2 & $(4.3884)$ & $(4.2859)$ \\
time3 & -0.0908 & -0.0915 \\
spellcount & $(0.0819)$ & $(0.0803)$ \\
GDP/cap. (log) & 0.0006 & 0.0006 \\
Constant & $(0.0005)$ & $(0.0005)$ \\
N & $-0.0342 *$ & -0.0260 \\
\hline & $(0.0206)$ & $(0.0169)$ \\
\hline
\end{tabular}




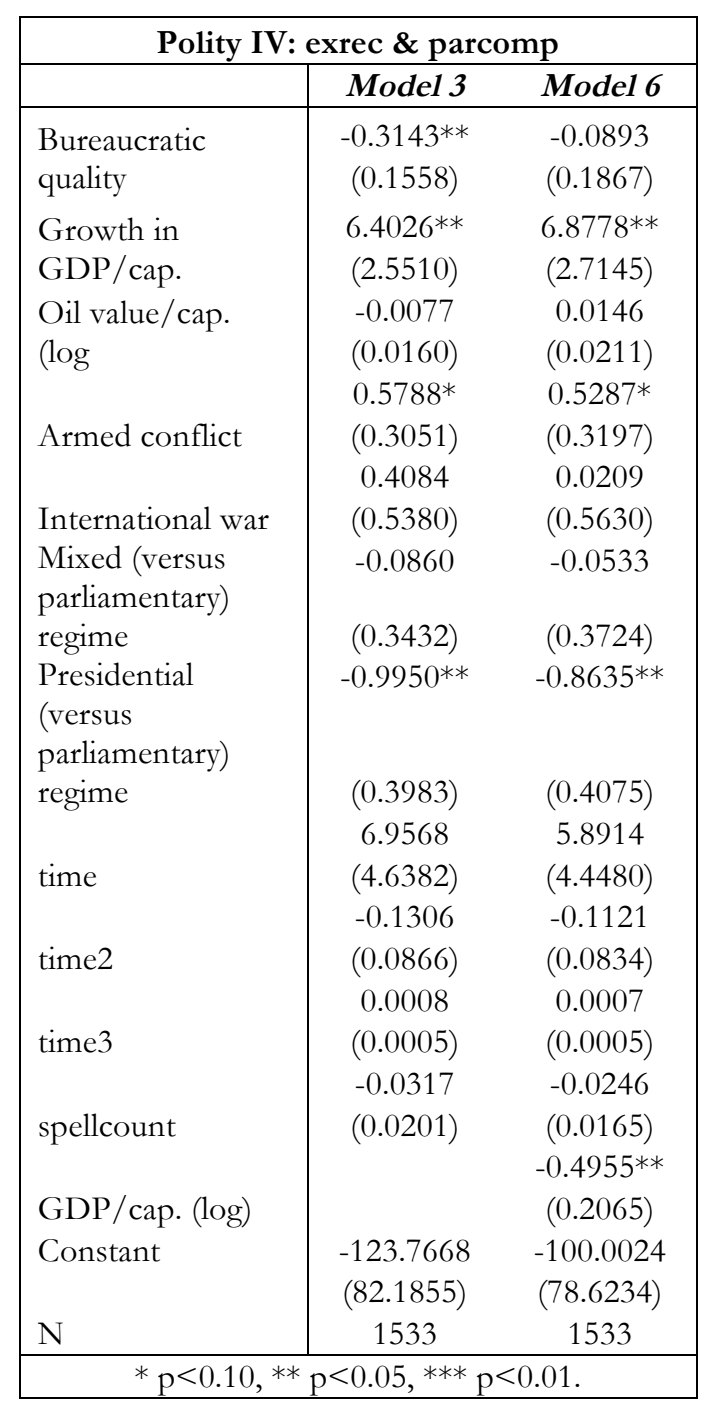

Polity IV: HYB; thresholds: 6 \& -6 not able to replicate (not concave)

\begin{tabular}{|l|cc|}
\hline \multicolumn{3}{|c|}{ Polity IV: HYB; thresholds: 8 \& 1 } \\
\hline & Model 3 & Model 6 \\
\hline Bureaucratic & -0.8159 & -0.6440 \\
quality & $(0.6685)$ & $(0.6374)$ \\
Growth in & -3.6909 & -4.1667 \\
GDP/cap. & $(7.3453)$ & $(7.8874)$ \\
Oil value/cap. & 0.0094 & 0.0260 \\
(log & $(0.0409)$ & $(0.0522)$ \\
& 1.2333 & 1.0970 \\
Armed conflict & $(0.7942)$ & $(0.7810)$ \\
& 0.0000 & 0.0000 \\
International war & $()$. & $()$. \\
Mixed (versus & -0.4550 & -0.7036 \\
parliamentary) & & \\
regime & $(0.9536)$ & $(1.0315)$ \\
Presidential & -1.1424 & -1.0922 \\
(versus & & \\
parliamentary) & & \\
regime & $(1.0722)$ & $(0.9339)$ \\
& 5.7132 & 5.1124 \\
time & $(14.4152)$ & $(12.9796)$ \\
& -0.0993 & -0.0903 \\
time2 & $(0.2660)$ & $(0.2406)$ \\
& 0.0006 & 0.0005 \\
time3 & $(0.0016)$ & $(0.0015)$ \\
spellcount & -0.0087 & -0.0111 \\
GDP/cap. (log) & $(0.0341)$ & $(0.0337)$ \\
Constant & & -0.3849 \\
N & -109.3787 & -93.8902 \\
\hline \multicolumn{1}{|c|}{$* .10, * *$} & $<0.05, * * *$ & $<<0.01$. \\
\hline
\end{tabular}




\begin{tabular}{|c|c|c|}
\hline \multicolumn{3}{|c|}{ Przeworski et al. 2000} \\
\hline & Model 3 & Model 6 \\
\hline $\begin{array}{l}\text { Bureaucratic } \\
\text { quality }\end{array}$ & $\begin{array}{l}-0.1952 \\
(0.1915)\end{array}$ & $\begin{array}{c}0.0283 \\
(0.2305)\end{array}$ \\
\hline Growth in & $-5.5789 * *$ & $-5.7376 * *$ \\
\hline GDP/cap. & $(2.5331)$ & $(2.6903)$ \\
\hline Oil value/cap. & 0.0118 & 0.0359 \\
\hline \multirow[t]{2}{*}{$(\log$} & $(0.0200)$ & $(0.0270)$ \\
\hline & 0.1811 & 0.0997 \\
\hline \multirow[t]{2}{*}{ Armed conflict } & $(0.3785)$ & $(0.3906)$ \\
\hline & 0.4679 & 0.0915 \\
\hline International war & $(0.5530)$ & $(0.6096)$ \\
\hline $\begin{array}{l}\text { Mixed (versus } \\
\text { parliamentary) }\end{array}$ & -0.3613 & -0.2902 \\
\hline regime & $(0.4610)$ & $(0.4835)$ \\
\hline $\begin{array}{l}\text { Presidential } \\
\text { (versus } \\
\text { parliamentary) }\end{array}$ & -0.5129 & -0.4429 \\
\hline \multirow[t]{2}{*}{ regime } & $(0.4171)$ & $(0.4441)$ \\
\hline & -1.3197 & 0.2349 \\
\hline \multirow[t]{2}{*}{ time } & $(12.2715)$ & $(12.6325)$ \\
\hline & 0.0330 & -0.0004 \\
\hline \multirow[t]{2}{*}{ time2 } & $(0.2516)$ & $(0.2592)$ \\
\hline & -0.0003 & -0.0000 \\
\hline \multirow[t]{2}{*}{ time3 } & $(0.0017)$ & $(0.0018)$ \\
\hline & -0.0239 & -0.0196 \\
\hline \multirow[t]{2}{*}{ spellcount } & $(0.0192)$ & $(0.0193)$ \\
\hline & & $-0.5127^{*}$ \\
\hline GDP/cap. (log) & & $(0.2748)$ \\
\hline \multirow[t]{2}{*}{ Constant } & 14.9618 & -4.9848 \\
\hline & (198.6181) & $(204.1642)$ \\
\hline $\mathrm{N}$ & 953 & 953 \\
\hline
\end{tabular}

\begin{tabular}{|l|cc|}
\hline \multicolumn{3}{|c|}{ Reich 2002 } \\
\hline & Mode1 3 & Model 6 \\
\hline Bureaucratic & -0.2258 & 0.1172 \\
quality & $(0.2404)$ & $(0.3197)$ \\
Growth in & 6.0307 & 6.3582 \\
GDP/cap. & $(4.1149)$ & $(4.4494)$ \\
Oil value/cap. & 0.0087 & 0.0419 \\
(log & $(0.0257)$ & $(0.0367)$ \\
& 0.7401 & 0.7337 \\
Armed conflict & $(0.5521)$ & $(0.6030)$ \\
International & 0.0000 & 0.0000 \\
war & $()$. & $()$. \\
Mixed (versus & 0.4145 & 0.7194 \\
parliamentary) & & \\
regime & $(0.6418)$ & $(0.7519)$ \\
Presidential & -0.6524 & -0.3281 \\
(versus & & \\
parliamentary) & & \\
regime & $(0.6021)$ & $(0.6495)$ \\
time & 79.2470 & 109.1262 \\
time2 & $(61.7239)$ & $(73.0617)$ \\
time3 & -1.6482 & -2.2796 \\
spellcount & $(1.2870)$ & $(1.5229)$ \\
GDP/cap. (log) & 0.0114 & 0.0158 \\
Constant & $(0.0089)$ & $(0.0106)$ \\
N & -0.0394 & -0.0329 \\
\hline \multicolumn{1}{|c|}{$* 0.0435)$} & $(0.0368)$ \\
\hline
\end{tabular}

\begin{tabular}{|l|cc|}
\hline \multicolumn{3}{|c|}{ Reich 2002: HYB } \\
\hline & Model 3 & Model 6 \\
\hline Bureaucratic & -0.2842 & 0.1354 \\
quality & $(0.4542)$ & $(0.6281)$ \\
Growth in & -2.1272 & -1.9118 \\
GDP/cap. & $(7.0986)$ & $(8.2481)$ \\
Oil value/cap. & -0.0026 & 0.1180 \\
log & $(0.0428)$ & $(0.1275)$ \\
& 0.9778 & 0.7513 \\
Armed conflict & $(0.7919)$ & $(0.8330)$ \\
International & 0.0000 & 0.0000 \\
war & $()$. & $()$. \\
Mixed (versus & 0.6068 & 0.6675 \\
parliamentary) & & \\
regime & $(0.7693)$ & $(1.0132)$ \\
Presidential & 0.0000 & 0.0000 \\
(versus & & \\
parliamentary) & & \\
regime & $()$. & $()$. \\
time & 49.5253 & 103.2930 \\
time2 & $(178.6271)$ & $(215.9438)$ \\
time3 & -0.9802 & -2.0991 \\
spellcount & $(3.6563)$ & $(4.4264)$ \\
GDP/cap. (log) & 0.0065 & 0.0142 \\
Constant & $(0.0249)$ & $(0.0302)$ \\
N & -0.0093 & 0.0064 \\
\hline \multicolumn{1}{|c|}{ p $<0.05, * * *$} & $<0.01$. \\
\hline
\end{tabular}




\begin{tabular}{|c|c|c|}
\hline \multicolumn{3}{|c|}{ Svolik 2012} \\
\hline & Model 3 & Model 6 \\
\hline $\begin{array}{l}\text { Bureaucratic } \\
\text { quality }\end{array}$ & $\begin{array}{l}-0.3194 \\
(0.2323)\end{array}$ & $\begin{array}{l}-0.1903 \\
(0.2633)\end{array}$ \\
\hline Growth in & -1.2695 & -1.0144 \\
\hline GDP/cap. & $(3.0296)$ & $(3.1294)$ \\
\hline \multirow{3}{*}{$\begin{array}{l}\text { Oil value/cap. } \\
\text { (log }\end{array}$} & 0.0094 & 0.0273 \\
\hline & $(0.0240)$ & $(0.0325)$ \\
\hline & 0.3168 & 0.2632 \\
\hline \multirow[t]{2}{*}{ Armed conflict } & $(0.4471)$ & $(0.4613)$ \\
\hline & 0.5432 & 0.3199 \\
\hline International war & $(0.6341)$ & $(0.6815)$ \\
\hline $\begin{array}{l}\text { Mixed (versus } \\
\text { parliamentary) }\end{array}$ & 0.0085 & 0.0379 \\
\hline regime & $(0.5381)$ & $(0.5995)$ \\
\hline $\begin{array}{l}\text { Presidential } \\
\text { (versus } \\
\text { parliamentary) }\end{array}$ & -0.5055 & -0.4389 \\
\hline \multirow[t]{2}{*}{ regime } & $(0.5112)$ & $(0.5524)$ \\
\hline & 3.0406 & 3.2005 \\
\hline \multirow[t]{2}{*}{ time } & $(7.4849)$ & $(7.6290)$ \\
\hline & -0.0547 & -0.0583 \\
\hline \multirow[t]{2}{*}{ time2 } & $(0.1440)$ & $(0.1468)$ \\
\hline & 0.0003 & 0.0003 \\
\hline \multirow[t]{2}{*}{ time3 } & $(0.0009)$ & $(0.0009)$ \\
\hline & -0.0188 & -0.0117 \\
\hline \multirow[t]{2}{*}{ spellcount } & $(0.0187)$ & (0.0197) \\
\hline & & -0.3843 \\
\hline GDP/cap. (log) & & $(0.3227)$ \\
\hline \multirow[t]{2}{*}{ Constant } & -57.4425 & -57.0029 \\
\hline & (128.9828) & $(131.4416)$ \\
\hline $\mathrm{N}$ & 1309 & 1309 \\
\hline
\end{tabular}

\begin{tabular}{|c|c|c|}
\hline \multicolumn{3}{|c|}{ Ulfelder 2012} \\
\hline & Model 3 & Model 6 \\
\hline $\begin{array}{l}\text { Bureaucratic } \\
\text { quality }\end{array}$ & $\begin{array}{c}-0.3338^{* * *} \\
(0.1105)\end{array}$ & $\begin{array}{l}-0.0850 \\
(0.1335)\end{array}$ \\
\hline Growth in & 2.1145 & 2.6429 \\
\hline GDP/cap. & (1.6194) & $(1.7043)$ \\
\hline Oil value/cap. & -0.0077 & 0.0067 \\
\hline$(\log$ & $(0.0103)$ & $(0.0121)$ \\
\hline & $0.4281 * *$ & 0.2806 \\
\hline Armed conflict & $(0.2167)$ & $(0.2261)$ \\
\hline & 0.2983 & -0.1451 \\
\hline International war & $(0.5004)$ & $(0.5111)$ \\
\hline $\begin{array}{l}\text { Mixed (versus } \\
\text { parliamentary) }\end{array}$ & 0.0207 & -0.0049 \\
\hline regime & $(0.2724)$ & $(0.2949)$ \\
\hline $\begin{array}{l}\text { Presidential } \\
\text { (versus } \\
\text { parliamentary) }\end{array}$ & -0.1853 & -0.1085 \\
\hline regime & $(0.2369)$ & $(0.2500)$ \\
\hline & 3.8823 & 4.4816 \\
\hline time & $(3.1487)$ & $(3.1275)$ \\
\hline & -0.0725 & -0.0857 \\
\hline time2 & $(0.0585)$ & $(0.0583)$ \\
\hline & 0.0005 & 0.0005 \\
\hline time3 & $(0.0004)$ & $(0.0004)$ \\
\hline & -0.0122 & -0.0042 \\
\hline spellcount & $(0.0080)$ & $(0.0077)$ \\
\hline & & $-0.4783^{* * *}$ \\
\hline GDP/cap. (log) & & $(0.1281)$ \\
\hline Constant & -70.5121 & -75.6263 \\
\hline & $(56.0883)$ & $(55.4517)$ \\
\hline $\mathrm{N}$ & 1425 & 1425 \\
\hline
\end{tabular}

\begin{tabular}{|c|c|c|}
\hline \multicolumn{3}{|c|}{ Wahman et al. 2013} \\
\hline & Model 3 & Model 6 \\
\hline $\begin{array}{l}\text { Bureaucratic } \\
\text { quality }\end{array}$ & $\begin{array}{c}-0.3212^{* *} \\
(0.1285)\end{array}$ & $\begin{array}{l}-0.0606 \\
(0.1542)\end{array}$ \\
\hline Growth in & -2.3730 & -1.6732 \\
\hline GDP/cap. & $(2.1909)$ & $(2.3879)$ \\
\hline Oil value/cap. & -0.0017 & 0.0150 \\
\hline \multirow[t]{2}{*}{$(\log$} & $(0.0123)$ & $(0.0148)$ \\
\hline & $0.5159 *$ & 0.3658 \\
\hline Armed conflict & $(0.2744)$ & $(0.2991)$ \\
\hline International & 0.6383 & 0.0110 \\
\hline war & $(0.4776)$ & $(0.5425)$ \\
\hline $\begin{array}{l}\text { Mixed (versus } \\
\text { parliamentary) }\end{array}$ & -0.4051 & -0.5919 \\
\hline regime & $(0.3415)$ & $(0.3976)$ \\
\hline $\begin{array}{l}\text { Presidential } \\
\text { (versus } \\
\text { parliamentary) }\end{array}$ & -0.1739 & -0.1675 \\
\hline \multirow[t]{2}{*}{ regime } & $(0.2694)$ & $(0.2926)$ \\
\hline & $10.0368 * *$ & $10.7524 * *$ \\
\hline \multirow[t]{2}{*}{ time } & $(4.4271)$ & $(4.5604)$ \\
\hline & $-0.1834 * *$ & $-0.1984 * *$ \\
\hline \multirow[t]{2}{*}{ time2 } & $(0.0814)$ & $(0.0839)$ \\
\hline & $0.0011 * *$ & $0.0012^{* *}$ \\
\hline \multirow[t]{2}{*}{ time3 } & $(0.0005)$ & $(0.0005)$ \\
\hline & $-0.0155^{*}$ & -0.0074 \\
\hline \multirow[t]{2}{*}{ spellcount } & $(0.0084)$ & $(0.0086)$ \\
\hline & & $-0.6006^{* * *}$ \\
\hline GDP/cap. (log) & & $(0.1883)$ \\
\hline Constant & $\begin{array}{c}-182.6619 * * \\
(79.7948)\end{array}$ & $\begin{array}{c}-189.1906^{* *} \\
(81.8194)\end{array}$ \\
\hline $\mathrm{N}$ & 1395 & 1395 \\
\hline \multicolumn{3}{|c|}{$* \mathrm{p}<0.10, * * \mathrm{p}<0.05, * * * \mathrm{p}<0.01$} \\
\hline
\end{tabular}


Appendix VII: Robustness Checks: Houle 2009

\begin{tabular}{|c|c|c|c|}
\hline & $\begin{array}{c}\text { Bernhard et } \\
\text { al. } 2001\end{array}$ & $\begin{array}{c}\text { Boix et al. } \\
2013\end{array}$ & $\begin{array}{c}\text { Cheibub et } \\
\text { al. } 2010\end{array}$ \\
\hline Capital share & $\begin{array}{c}1.8185 \\
(1.2569)\end{array}$ & $\begin{array}{l}3.2668^{*} \\
(1.7682)\end{array}$ & $\begin{array}{l}\text { convergence } \\
\text { not achieved }\end{array}$ \\
\hline Log GDP pc & $\begin{array}{c}-2.9407 * * * \\
(0.7269)\end{array}$ & $\begin{array}{c}-1.8399 * * \\
(0.8685)\end{array}$ & \\
\hline Growth & $\begin{array}{c}0.0028 \\
(0.0046)\end{array}$ & $\begin{array}{l}-0.0080 \\
(0.0189)\end{array}$ & \\
\hline Oil & $\begin{array}{l}-0.3467 \\
(0.6011)\end{array}$ & $\begin{array}{l}-1.5851 \\
(0.9896)\end{array}$ & \\
\hline Muslim & $\begin{array}{c}0.0018 \\
(0.0066)\end{array}$ & $\begin{array}{c}0.0079 \\
(0.0082)\end{array}$ & \\
\hline Catholic & $\begin{array}{c}0.0125 \\
(0.0077)\end{array}$ & $\begin{array}{c}0.0210^{* *} \\
(0.0087)\end{array}$ & \\
\hline Protestant & $\begin{array}{l}-0.0053 \\
(0.0170)\end{array}$ & $\begin{array}{c}0.0155 \\
(0.0169)\end{array}$ & \\
\hline Ethnic fractionalization & $\begin{array}{c}0.0067 \\
(0.0077)\end{array}$ & $\begin{array}{l}-0.0176 \\
(0.0125)\end{array}$ & \\
\hline Religious fractionalization & $\begin{array}{c}-0.0194 * \\
(0.0105)\end{array}$ & $\begin{array}{c}-0.0270^{* *} \\
(0.0112)\end{array}$ & \\
\hline British colony & $\begin{array}{c}0.1462 \\
(0.3792)\end{array}$ & $\begin{array}{c}-1.5224 * * * \\
(0.5616)\end{array}$ & \\
\hline New country & $\begin{array}{l}-0.6351 \\
(0.5924)\end{array}$ & $\begin{array}{l}-0.1482 \\
(0.4945)\end{array}$ & \\
\hline Past transitions & $\begin{array}{c}0.4270 * * * \\
(0.1521)\end{array}$ & $\begin{array}{c}1.2705^{* * *} \\
(0.2397)\end{array}$ & \\
\hline$\%$ democracies in the world & $\begin{array}{l}-0.2056 \\
(3.4327)\end{array}$ & $\begin{array}{l}-3.4395 \\
(4.2568)\end{array}$ & \\
\hline Presidentialism & $\begin{array}{c}-1.2566 * * * \\
(0.3802)\end{array}$ & $\begin{array}{c}0.0000 \\
(.)\end{array}$ & \\
\hline Latin America & $\begin{array}{l}-0.1704 \\
(0.6940)\end{array}$ & $\begin{array}{c}0.1691 \\
(0.6687)\end{array}$ & \\
\hline Eastern Europe & $\begin{array}{l}-0.8570 \\
(0.9623)\end{array}$ & $\begin{array}{l}-0.1152 \\
(0.8228)\end{array}$ & \\
\hline Western Europe & $\begin{array}{c}0.0000 \\
(.)\end{array}$ & $\begin{array}{c}-1.8853^{*} \\
(0.9935)\end{array}$ & \\
\hline Africa & $\begin{array}{c}0.3048 \\
(0.4291)\end{array}$ & $\begin{array}{c}0.4924 \\
(0.4963)\end{array}$ & \\
\hline Middle East & $\begin{array}{c}0.8717 \\
(0.9011)\end{array}$ & $\begin{array}{c}0.0280 \\
(0.8453)\end{array}$ & \\
\hline Decade: 60ies & $\begin{array}{l}-0.1606 \\
(0.7851)\end{array}$ & $\begin{array}{c}0.5035 \\
(1.0785)\end{array}$ & \\
\hline Decade: 70ies & $\begin{array}{c}0.3329 \\
(0.9587)\end{array}$ & $\begin{array}{c}0.1727 \\
(1.2413)\end{array}$ & \\
\hline Decade: 80 ies & $\begin{array}{l}-0.1133 \\
(0.7523)\end{array}$ & $\begin{array}{c}0.1537 \\
(0.9120)\end{array}$ & \\
\hline Constant & $\begin{array}{c}7.8642 * * \\
(3.2862)\end{array}$ & $\begin{array}{c}6.0484 \\
(4.1392)\end{array}$ & \\
\hline $\mathrm{N}$ & 856 & 1215 & \\
\hline Pseudo-R2 & 0.331 & 0.632 & \\
\hline Log Likelihood & -82.489 & -51.701 & \\
\hline
\end{tabular}




\begin{tabular}{|c|c|c|c|c|c|c|}
\hline & \multicolumn{3}{|c|}{ Freedom } & \multicolumn{3}{|c|}{ Freedom } \\
\hline & $\begin{array}{c}\text { Freedom } \\
\text { House } \\
\text { (FIW: free) }\end{array}$ & $\begin{array}{c}\text { House } \\
\text { (FIW: partly } \\
\text { free) }\end{array}$ & $\begin{array}{c}\text { Freedom } \\
\text { House (PR: } \\
\text { free) }\end{array}$ & $\begin{array}{c}\text { Freedom } \\
\text { House (PR: } \\
\text { partly free) }\end{array}$ & $\begin{array}{l}\text { House } \\
\text { (FIW: } \\
\text { hybrid) }\end{array}$ & $\begin{array}{c}\text { Freedom } \\
\text { House (PR: } \\
\text { hybrid) }\end{array}$ \\
\hline Capital share & $\begin{array}{c}3.4348^{* * *} \\
(1.1849)\end{array}$ & $\begin{array}{l}1.6267 * \\
(0.8422)\end{array}$ & $\begin{array}{c}3.3438^{* * * *} \\
(1.0799)\end{array}$ & $\begin{array}{c}1.2776 \\
(0.7794)\end{array}$ & $\begin{array}{c}\text { outcome } \\
\text { does not vary }\end{array}$ & $\begin{array}{c}\text { outcome } \\
\text { does not vary }\end{array}$ \\
\hline Log GDP pc & $\begin{array}{c}-2.1449 * * * \\
(0.5272)\end{array}$ & $\begin{array}{c}-0.7358^{* *} \\
(0.3346)\end{array}$ & $\begin{array}{c}-1.8226 * * * \\
(0.4890)\end{array}$ & $\begin{array}{c}-0.8368 * * * \\
(0.3086)\end{array}$ & & \\
\hline Growth & $\begin{array}{c}-0.0502^{* * *} \\
(0.0195)\end{array}$ & $\begin{array}{c}-0.0224^{*} \\
(0.0124)\end{array}$ & $\begin{array}{c}-0.0669 * * * \\
(0.0195)\end{array}$ & $\begin{array}{l}-0.0085 \\
(0.0140)\end{array}$ & & \\
\hline Oil & $\begin{array}{c}0.1040 \\
(0.4122)\end{array}$ & $\begin{array}{c}0.0974 \\
(0.3154)\end{array}$ & $\begin{array}{l}-0.1915 \\
(0.4073)\end{array}$ & $\begin{array}{c}0.7441 * * \\
(0.3026)\end{array}$ & & \\
\hline Muslim & $\begin{array}{l}-0.0037 \\
(0.0065)\end{array}$ & $\begin{array}{c}0.0058 \\
(0.0044)\end{array}$ & $\begin{array}{c}0.0037 \\
(0.0059)\end{array}$ & $\begin{array}{c}0.0000 \\
(0.0038)\end{array}$ & & \\
\hline Catholic & $\begin{array}{c}0.0089 \\
(0.0081)\end{array}$ & $\begin{array}{c}0.0098 \\
(0.0062)\end{array}$ & $\begin{array}{c}0.0118 \\
(0.0079)\end{array}$ & $\begin{array}{c}0.0008 \\
(0.0056)\end{array}$ & & \\
\hline Protestant & $\begin{array}{c}0.0052 \\
(0.0097)\end{array}$ & $\begin{array}{c}0.0103 \\
(0.0073)\end{array}$ & $\begin{array}{l}-0.0025 \\
(0.0101)\end{array}$ & $\begin{array}{c}0.0066 \\
(0.0061)\end{array}$ & & \\
\hline Ethnic fractionalization & $\begin{array}{l}-0.0001 \\
(0.0074)\end{array}$ & $\begin{array}{l}-0.0066 \\
(0.0048)\end{array}$ & $\begin{array}{c}0.0054 \\
(0.0067)\end{array}$ & $\begin{array}{c}0.0012 \\
(0.0044)\end{array}$ & & \\
\hline Religious fractionalization & $\begin{array}{c}0.0063 \\
(0.0105)\end{array}$ & $\begin{array}{l}-0.0066 \\
(0.0068)\end{array}$ & $\begin{array}{l}-0.0104 \\
(0.0107)\end{array}$ & $\begin{array}{l}-0.0009 \\
(0.0064)\end{array}$ & & \\
\hline British colony & $\begin{array}{l}-0.4971 \\
(0.4672)\end{array}$ & $\begin{array}{c}-0.3784 * \\
(0.2101)\end{array}$ & $\begin{array}{c}0.2768 \\
(0.4510)\end{array}$ & $\begin{array}{c}-0.3320^{*} \\
(0.1961)\end{array}$ & & \\
\hline New country & $\begin{array}{c}0.3931 \\
(0.4784)\end{array}$ & $\begin{array}{l}-0.1166 \\
(0.2544)\end{array}$ & $\begin{array}{c}-0.9270^{*} \\
(0.5344)\end{array}$ & $\begin{array}{c}0.1140 \\
(0.2557)\end{array}$ & & \\
\hline Past transitions & $\begin{array}{l}0.2237^{*} \\
(0.1359)\end{array}$ & $\begin{array}{c}0.1370 \\
(0.1032)\end{array}$ & $\begin{array}{c}0.1517 \\
(0.1116)\end{array}$ & $\begin{array}{c}0.2496 * * \\
(0.0973)\end{array}$ & & \\
\hline$\%$ democracies in the world & $\begin{array}{c}0.6972 \\
(2.8480)\end{array}$ & $\begin{array}{c}0.9005 \\
(2.3572)\end{array}$ & $\begin{array}{c}1.6032 \\
(2.5673)\end{array}$ & $\begin{array}{c}1.8264 \\
(2.2507)\end{array}$ & & \\
\hline Presidentialism & $\begin{array}{c}-1.0899 * * \\
(0.4320)\end{array}$ & $\begin{array}{c}-0.9259 * * * \\
(0.3165)\end{array}$ & $\begin{array}{c}-0.8771 * * \\
(0.3849)\end{array}$ & $\begin{array}{c}-1.6234 * * * \\
(0.3932)\end{array}$ & & \\
\hline Latin America & $\begin{array}{l}-0.5108 \\
(0.4983)\end{array}$ & $\begin{array}{l}-0.3441 \\
(0.4727)\end{array}$ & $\begin{array}{c}-1.0206^{*} \\
(0.5290)\end{array}$ & $\begin{array}{c}0.2944 \\
(0.4071)\end{array}$ & & \\
\hline Eastern Europe & $\begin{array}{l}-0.7028 \\
(0.6614)\end{array}$ & $\begin{array}{l}-0.1438 \\
(0.5365)\end{array}$ & $\begin{array}{c}-1.8764 * * \\
(0.7570)\end{array}$ & $\begin{array}{c}0.0000 \\
(.)\end{array}$ & & \\
\hline Western Europe & $\begin{array}{l}-1.0737 \\
(0.7987)\end{array}$ & $\begin{array}{c}0.0000 \\
(.)\end{array}$ & $\begin{array}{c}-1.8466 * * \\
(0.8056)\end{array}$ & $\begin{array}{c}0.0000 \\
(.)\end{array}$ & & \\
\hline Africa & $\begin{array}{l}-0.3245 \\
(0.4258)\end{array}$ & $\begin{array}{c}0.1741 \\
(0.3101)\end{array}$ & $\begin{array}{l}-0.4442 \\
(0.4036)\end{array}$ & $\begin{array}{c}0.3895 \\
(0.2513)\end{array}$ & & \\
\hline Middle East & $\begin{array}{c}0.2650 \\
(0.8745)\end{array}$ & $\begin{array}{c}0.9938^{* * *} \\
(0.3662)\end{array}$ & $\begin{array}{l}-0.2203 \\
(0.7508)\end{array}$ & $\begin{array}{c}0.7904 * * \\
(0.3329)\end{array}$ & & \\
\hline Decade: 60ies & $\begin{array}{c}0.0000 \\
(.)\end{array}$ & $\begin{array}{c}0.0000 \\
(.)\end{array}$ & $\begin{array}{c}0.0000 \\
(.)\end{array}$ & $\begin{array}{c}0.0000 \\
(.)\end{array}$ & & \\
\hline Decade: 70ies & $\begin{array}{c}0.3004 \\
(0.7892)\end{array}$ & $\begin{array}{c}0.2845 \\
(0.6577)\end{array}$ & $\begin{array}{c}0.2117 \\
(0.7206)\end{array}$ & $\begin{array}{c}0.6724 \\
(0.6355)\end{array}$ & & \\
\hline Decade: 80ies & $\begin{array}{l}-0.3661 \\
(0.5959)\end{array}$ & $\begin{array}{c}0.2387 \\
(0.4873)\end{array}$ & $\begin{array}{l}-0.2128 \\
(0.5271)\end{array}$ & $\begin{array}{c}0.6927 \\
(0.4669)\end{array}$ & & \\
\hline Constant & $\begin{array}{c}3.3319 \\
(3.0270)\end{array}$ & $\begin{array}{l}-0.5967 \\
(2.0026)\end{array}$ & $\begin{array}{c}3.3167 \\
(2.8436)\end{array}$ & $\begin{array}{l}-1.2550 \\
(1.8775)\end{array}$ & & \\
\hline $\mathrm{N}$ & 1053 & 1399 & 1076 & 1162 & & \\
\hline Pseudo-R2 & 0.385 & 0.164 & 0.367 & 0.181 & & \\
\hline Log Likelihood & -96.434 & -151.823 & -116.322 & -173.928 & & \\
\hline
\end{tabular}




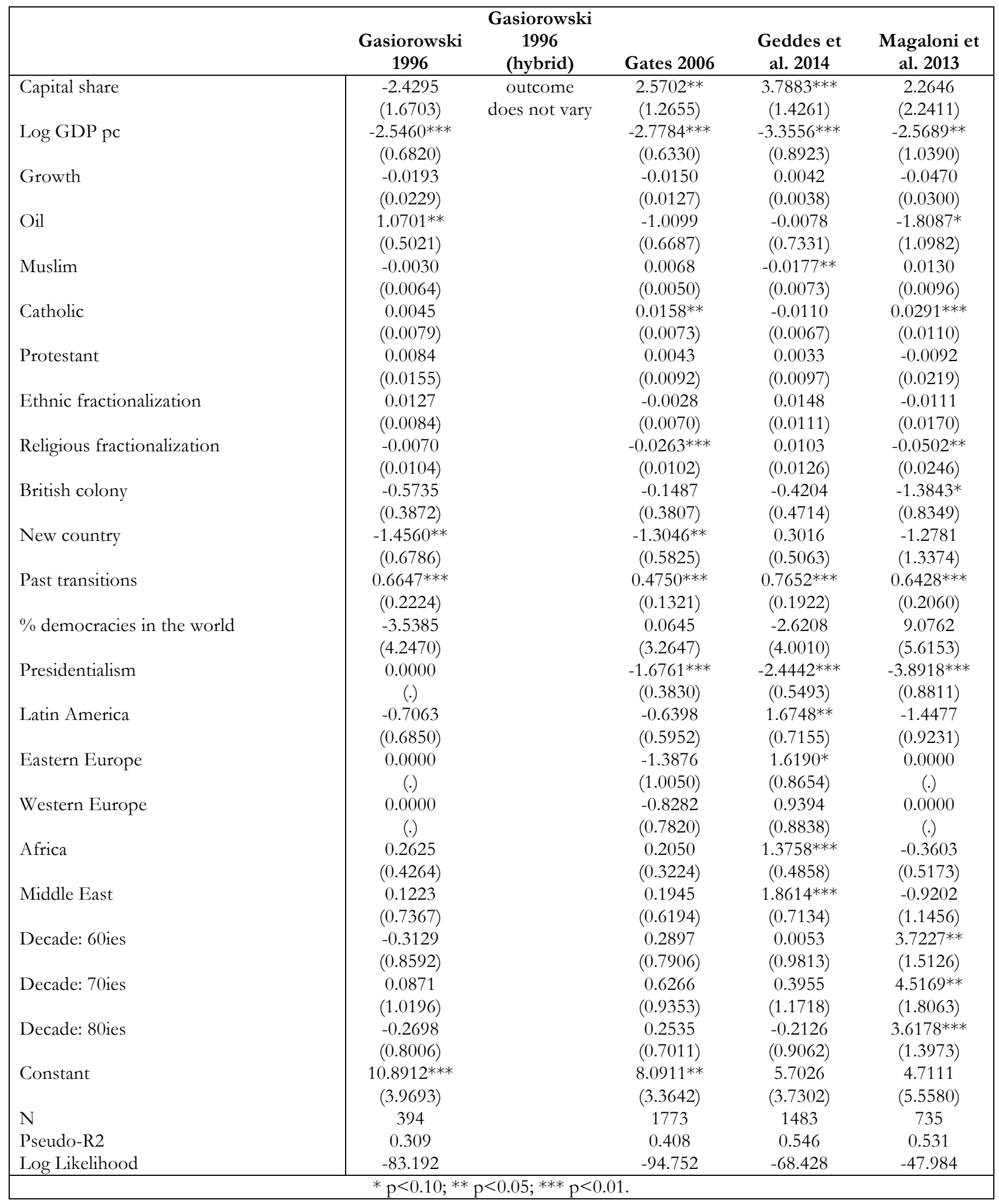




\begin{tabular}{|c|c|c|c|c|c|c|c|}
\hline & $\begin{array}{c}\text { Polity IV } \\
\text { (AN=AUT) }\end{array}$ & $\begin{array}{c}\text { Polity IV } \\
(\text { AN=DEM) }\end{array}$ & $\begin{array}{c}\text { Polity IV } \\
\text { (threshold: } \\
7 \text { ) } \\
\end{array}$ & $\begin{array}{c}\text { Polity IV } \\
\text { (threshold: } \\
\text { 1) }\end{array}$ & $\begin{array}{c}\text { Polity IV } \\
\text { (exrec \& } \\
\text { parcomp) } \\
\end{array}$ & $\begin{array}{c}\text { Polity IV } \\
\text { (hybrid: } \\
6 \&-6 \text { ) } \\
\end{array}$ & $\begin{array}{c}\text { Polity IV } \\
(8 \& \text { \&) }\end{array}$ \\
\hline Capital share & 2.4145 & 0.8995 & 0.9004 & 1.7199 & $2.6252 *$ & $\begin{array}{l}\text { outcome } \\
\text { does not }\end{array}$ & $\begin{array}{l}\text { outcome } \\
\text { does not }\end{array}$ \\
\hline & $(1.4984)$ & $(0.9499)$ & (2.0491) & $(1.0844)$ & $(1.3584)$ & vary & vary \\
\hline Log GDP pc & $\begin{array}{c}-3.5152^{* * *} \\
(0.8242)\end{array}$ & $\begin{array}{c}-1.1087 * * * \\
(0.4280)\end{array}$ & $\begin{array}{c}-3.0869 * * * \\
(1.0691)\end{array}$ & $\begin{array}{c}-2.6645^{* * *} \\
(0.5960)\end{array}$ & $\begin{array}{c}-2.9542 * * * \\
(0.7462)\end{array}$ & & \\
\hline Growth & $\begin{array}{l}-0.0322 \\
(0.0255)\end{array}$ & $\begin{array}{l}-0.0030 \\
(0.0100)\end{array}$ & $\begin{array}{c}-0.0596^{*} \\
(0.0304)\end{array}$ & $\begin{array}{l}-0.0122 \\
(0.0102)\end{array}$ & $\begin{array}{l}-0.0064 \\
(0.0129)\end{array}$ & & \\
\hline Oil & $\begin{array}{l}-1.0787 \\
(0.7329)\end{array}$ & $\begin{array}{c}0.9174 * * * \\
(0.3480)\end{array}$ & $\begin{array}{l}-0.5642 \\
(0.7702)\end{array}$ & $\begin{array}{l}-0.6033 \\
(0.6017)\end{array}$ & $\begin{array}{l}-0.6649 \\
(0.6846)\end{array}$ & & \\
\hline Muslim & $\begin{array}{c}0.0030 \\
(0.0071)\end{array}$ & $\begin{array}{l}-0.0024 \\
(0.0042)\end{array}$ & $\begin{array}{l}0.0172 * \\
(0.0103)\end{array}$ & $\begin{array}{c}0.0015 \\
(0.0045)\end{array}$ & $\begin{array}{c}0.0018 \\
(0.0054)\end{array}$ & & \\
\hline Catholic & $\begin{array}{l}0.0176^{*} \\
(0.0102)\end{array}$ & $\begin{array}{l}-0.0010 \\
(0.0058)\end{array}$ & $\begin{array}{c}0.0367 * * * \\
(0.0137)\end{array}$ & $\begin{array}{c}0.0089 \\
(0.0062)\end{array}$ & $\begin{array}{c}0.0091 \\
(0.0082)\end{array}$ & & \\
\hline Protestant & $\begin{array}{c}0.0052 \\
(0.0160)\end{array}$ & $\begin{array}{l}-0.0017 \\
(0.0079)\end{array}$ & $\begin{array}{l}-0.0083 \\
(0.0195)\end{array}$ & $\begin{array}{c}0.0017 \\
(0.0079)\end{array}$ & $\begin{array}{l}0.0186^{*} \\
(0.0113)\end{array}$ & & \\
\hline Ethnic fractionalization & $\begin{array}{c}0.0142 \\
(0.0097)\end{array}$ & $\begin{array}{c}0.0030 \\
(0.0048)\end{array}$ & $\begin{array}{c}0.0091 \\
(0.0117)\end{array}$ & $\begin{array}{c}0.0015 \\
(0.0062)\end{array}$ & $\begin{array}{c}0.0092 \\
(0.0079)\end{array}$ & & \\
\hline Religious fractionalization & $\begin{array}{l}-0.0300^{*} \\
(0.0159)\end{array}$ & $\begin{array}{c}0.0026 \\
(0.0074)\end{array}$ & $\begin{array}{c}-0.0460^{* *} \\
(0.0190)\end{array}$ & $\begin{array}{l}-0.0156^{*} \\
(0.0082)\end{array}$ & $\begin{array}{l}-0.0161 \\
(0.0119)\end{array}$ & & \\
\hline British colony & $\begin{array}{l}-0.0695 \\
(0.5118)\end{array}$ & $\begin{array}{l}-0.3721 \\
(0.2364)\end{array}$ & $\begin{array}{l}-0.2991 \\
(0.6096)\end{array}$ & $\begin{array}{l}-0.1836 \\
(0.3285)\end{array}$ & $\begin{array}{l}-0.0404 \\
(0.4584)\end{array}$ & & \\
\hline New country & $\begin{array}{l}-0.6927 \\
(0.8246)\end{array}$ & $\begin{array}{c}0.3676 \\
(0.3743)\end{array}$ & $\begin{array}{l}-0.6238 \\
(1.0148)\end{array}$ & $\begin{array}{c}-0.8069^{*} \\
(0.4796)\end{array}$ & $\begin{array}{c}-1.5750 * * \\
(0.7301)\end{array}$ & & \\
\hline Past transitions & $\begin{array}{c}0.4495^{* * * *} \\
(0.1631)\end{array}$ & $\begin{array}{c}0.5207 * * * \\
(0.1248)\end{array}$ & $\begin{array}{c}0.2209 \\
(0.1681)\end{array}$ & $\begin{array}{c}0.3984 * * * \\
(0.1247)\end{array}$ & $\begin{array}{c}0.3587 * * \\
(0.1451)\end{array}$ & & \\
\hline$\%$ democracies in the world & $\begin{array}{l}-0.3366 \\
(3.6632)\end{array}$ & $\begin{array}{l}-5.0656^{*} \\
(2.9088)\end{array}$ & $\begin{array}{c}2.9215 \\
(4.0929)\end{array}$ & $\begin{array}{l}-0.8390 \\
(3.0413)\end{array}$ & $\begin{array}{c}0.7025 \\
(3.6200)\end{array}$ & & \\
\hline Presidentialism & $\begin{array}{c}-1.2182^{* * *} \\
(0.4399)\end{array}$ & $\begin{array}{c}0.0000 \\
(.)\end{array}$ & $\begin{array}{c}-2.0336 * * * \\
(0.5573)\end{array}$ & $\begin{array}{c}-1.3404 * * * \\
(0.3430)\end{array}$ & $\begin{array}{c}-1.2809 * * * \\
(0.3807)\end{array}$ & & \\
\hline Latin America & $\begin{array}{l}-0.8412 \\
(0.6626)\end{array}$ & $\begin{array}{c}0.2173 \\
(0.4866)\end{array}$ & $\begin{array}{l}-0.9647 \\
(0.7332)\end{array}$ & $\begin{array}{l}-0.1840 \\
(0.5140)\end{array}$ & $\begin{array}{l}-0.8119 \\
(0.7153)\end{array}$ & & \\
\hline Eastern Europe & $\begin{array}{c}0.0000 \\
(.)\end{array}$ & $\begin{array}{c}0.0000 \\
(.)\end{array}$ & $\begin{array}{c}0.0000 \\
(.)\end{array}$ & $\begin{array}{l}-0.4411 \\
(0.8845)\end{array}$ & $\begin{array}{l}-1.1501 \\
(1.2424)\end{array}$ & & \\
\hline Western Europe & $\begin{array}{l}-0.5135 \\
(1.0092)\end{array}$ & $\begin{array}{l}-0.2317 \\
(0.6435)\end{array}$ & $\begin{array}{l}-1.6591 \\
(1.0542)\end{array}$ & $\begin{array}{l}-0.1065 \\
(0.6513)\end{array}$ & $\begin{array}{c}0.0000 \\
(.)\end{array}$ & & \\
\hline Africa & $\begin{array}{c}0.0490 \\
(0.4063)\end{array}$ & $\begin{array}{l}0.4941 * \\
(0.2796)\end{array}$ & $\begin{array}{l}-0.6762 \\
(0.5157)\end{array}$ & $\begin{array}{c}0.3132 \\
(0.3063)\end{array}$ & $\begin{array}{c}0.5583 \\
(0.3733)\end{array}$ & & \\
\hline Middle East & $\begin{array}{c}0.7756 \\
(0.8384)\end{array}$ & $\begin{array}{c}0.8089 * * \\
(0.4078)\end{array}$ & $\begin{array}{c}0.2515 \\
(0.9033)\end{array}$ & $\begin{array}{c}0.5155 \\
(0.5835)\end{array}$ & $\begin{array}{c}0.1003 \\
(0.6799)\end{array}$ & & \\
\hline Decade: 60ies & $\begin{array}{c}0.2471 \\
(0.8552)\end{array}$ & $\begin{array}{l}-0.3075 \\
(0.6939)\end{array}$ & $\begin{array}{c}0.6549 \\
(0.9994)\end{array}$ & $\begin{array}{c}0.4518 \\
(0.7464)\end{array}$ & $\begin{array}{c}0.1715 \\
(0.8977)\end{array}$ & & \\
\hline Decade: 70ies & $\begin{array}{c}0.5889 \\
(1.0596)\end{array}$ & $\begin{array}{l}-0.0987 \\
(0.8092)\end{array}$ & $\begin{array}{c}0.9595 \\
(1.2358)\end{array}$ & $\begin{array}{c}0.8315 \\
(0.8838)\end{array}$ & $\begin{array}{c}1.0511 \\
(1.0373)\end{array}$ & & \\
\hline Decade: 80ies & $\begin{array}{c}0.3641 \\
(0.7827)\end{array}$ & $\begin{array}{l}-0.0279 \\
(0.6421)\end{array}$ & $\begin{array}{c}0.5967 \\
(0.8905)\end{array}$ & $\begin{array}{c}0.3613 \\
(0.6862)\end{array}$ & $\begin{array}{c}0.4000 \\
(0.7932)\end{array}$ & & \\
\hline Constant & $\begin{array}{c}9.7107 * * \\
(4.2709)\end{array}$ & $\begin{array}{c}2.3025 \\
(2.5867)\end{array}$ & $\begin{array}{l}9.0909 * \\
(5.4112)\end{array}$ & $\begin{array}{c}7.2263^{* *} \\
(2.9813)\end{array}$ & $\begin{array}{l}6.8936^{*} \\
(3.8103)\end{array}$ & & \\
\hline $\mathrm{N}$ & 1496 & 1680 & 1388 & 1879 & 989 & & \\
\hline Pseudo-R2 & 0.413 & 0.306 & 0.438 & 0.377 & 0.344 & & \\
\hline Log Likelihood & -69.806 & -136.247 & -58.770 & -115.832 & -85.862 & & \\
\hline
\end{tabular}




\begin{tabular}{|c|c|c|c|c|c|c|}
\hline & $\begin{array}{c}\text { Przeworski } \\
\text { et al. } 2000\end{array}$ & Reich 2002 & $\begin{array}{c}\text { Reich } 2002 \\
\text { (hybrid) }\end{array}$ & Svolik 2012 & $\begin{array}{c}\text { Ulfelder } \\
2012\end{array}$ & $\begin{array}{c}\text { Wahman et } \\
\text { al. } 2013\end{array}$ \\
\hline Capital share & $\begin{array}{l}\text { convergence } \\
\text { not achieved }\end{array}$ & $\begin{array}{c}-2.5996^{*} \\
(1.5091)\end{array}$ & $\begin{array}{c}\text { outcome } \\
\text { does not vary }\end{array}$ & $\begin{array}{c}0.3813 \\
(2.1519)\end{array}$ & $\begin{array}{c}1.2198 \\
(1.0589)\end{array}$ & $\begin{array}{c}1.7559 \\
(1.2451)\end{array}$ \\
\hline Log GDP pc & & $\begin{array}{c}-2.9109 * * * \\
(0.6469)\end{array}$ & & $\begin{array}{c}-2.7036^{* *} \\
(1.1742)\end{array}$ & $\begin{array}{c}-2.2950^{* * *} \\
(0.4974)\end{array}$ & $\begin{array}{c}-2.1621 * * * \\
(0.6578)\end{array}$ \\
\hline Growth & & $\begin{array}{l}-0.0150 \\
(0.0215)\end{array}$ & & $\begin{array}{c}0.0000 \\
(0.0077)\end{array}$ & $\begin{array}{c}0.0111^{* *} \\
(0.0051)\end{array}$ & $\begin{array}{c}-0.0439 * \\
(0.0238)\end{array}$ \\
\hline Oil & & $\begin{array}{c}1.2994 * * * \\
(0.4931)\end{array}$ & & $\begin{array}{l}-0.9851 \\
(0.9406)\end{array}$ & $\begin{array}{l}-0.3149 \\
(0.5656)\end{array}$ & $\begin{array}{l}-0.0597 \\
(0.4951)\end{array}$ \\
\hline Muslim & & $\begin{array}{l}-0.0054 \\
(0.0059)\end{array}$ & & $\begin{array}{c}0.0294 * * \\
(0.0124)\end{array}$ & $\begin{array}{c}0.0001 \\
(0.0045)\end{array}$ & $\begin{array}{c}0.0133 * * \\
(0.0063)\end{array}$ \\
\hline Catholic & & $\begin{array}{c}0.0035 \\
(0.0071)\end{array}$ & & $\begin{array}{c}0.0290 * * \\
(0.0115)\end{array}$ & $\begin{array}{c}0.0056 \\
(0.0055)\end{array}$ & $\begin{array}{c}0.0214 * * \\
(0.0096)\end{array}$ \\
\hline Protestant & & $\begin{array}{l}-0.0015 \\
(0.0118)\end{array}$ & & $\begin{array}{c}0.0189 \\
(0.0210)\end{array}$ & $\begin{array}{l}-0.0017 \\
(0.0085)\end{array}$ & $\begin{array}{l}-0.0044 \\
(0.0153)\end{array}$ \\
\hline Ethnic fractionalization & & $\begin{array}{c}0.0165^{* *} \\
(0.0080)\end{array}$ & & $\begin{array}{c}-0.0230^{*} \\
(0.0124)\end{array}$ & $\begin{array}{c}0.0053 \\
(0.0057)\end{array}$ & $\begin{array}{l}-0.0074 \\
(0.0080)\end{array}$ \\
\hline Religious fractionalization & & $\begin{array}{l}-0.0065 \\
(0.0096)\end{array}$ & & $\begin{array}{l}-0.0318 \\
(0.0213)\end{array}$ & $\begin{array}{l}-0.0095 \\
(0.0071)\end{array}$ & $\begin{array}{c}-0.0249 * \\
(0.0135)\end{array}$ \\
\hline British colony & & $\begin{array}{l}-0.5073 \\
(0.3515)\end{array}$ & & $\begin{array}{l}-1.0409 \\
(0.7617)\end{array}$ & $\begin{array}{l}-0.1112 \\
(0.3071)\end{array}$ & $\begin{array}{l}-0.0758 \\
(0.5370)\end{array}$ \\
\hline New country & & $\begin{array}{c}-1.2302 * * \\
(0.5977)\end{array}$ & & $\begin{array}{c}-2.3255^{*} \\
(1.2724)\end{array}$ & $\begin{array}{l}-0.5898 \\
(0.4413)\end{array}$ & $\begin{array}{l}-0.6243 \\
(0.5370)\end{array}$ \\
\hline Past transitions & & $\begin{array}{c}0.7455^{* * *} \\
(0.1992)\end{array}$ & & $\begin{array}{c}0.9076 * * * \\
(0.2165)\end{array}$ & $\begin{array}{c}0.3614 * * * \\
(0.1214)\end{array}$ & $\begin{array}{c}0.0243 \\
(0.1501)\end{array}$ \\
\hline$\%$ democracies in the world & & $\begin{array}{l}-6.9307^{*} \\
(3.6705)\end{array}$ & & $\begin{array}{l}-6.6763 \\
(4.5636)\end{array}$ & $\begin{array}{l}-3.2714 \\
(2.8794)\end{array}$ & $\begin{array}{c}3.5490 \\
(3.2937)\end{array}$ \\
\hline Presidentialism & & $\begin{array}{c}0.0000 \\
(.)\end{array}$ & & $\begin{array}{c}-4.3208^{* * *} \\
(0.9233)\end{array}$ & $\begin{array}{c}-1.3539 * * * \\
(0.3076)\end{array}$ & $\begin{array}{c}-1.1501 * * * \\
(0.3840)\end{array}$ \\
\hline Latin America & & $\begin{array}{l}-0.4524 \\
(0.6389)\end{array}$ & & $\begin{array}{l}-0.0083 \\
(0.9158)\end{array}$ & $\begin{array}{l}-0.0931 \\
(0.4694)\end{array}$ & $\begin{array}{l}-0.8672 \\
(0.5588)\end{array}$ \\
\hline Eastern Europe & & $\begin{array}{c}0.0000 \\
(.)\end{array}$ & & $\begin{array}{c}0.0000 \\
(.)\end{array}$ & $\begin{array}{l}-0.1632 \\
(0.7791)\end{array}$ & $\begin{array}{c}-1.3752^{*} \\
(0.7572)\end{array}$ \\
\hline Western Europe & & $\begin{array}{c}-1.9963 * * \\
(0.9066)\end{array}$ & & $\begin{array}{c}-2.6665^{*} \\
(1.4097)\end{array}$ & $\begin{array}{l}-0.5348 \\
(0.6315)\end{array}$ & $\begin{array}{c}0.0000 \\
(.)\end{array}$ \\
\hline Africa & & $\begin{array}{c}0.4456 \\
(0.3996)\end{array}$ & & $\begin{array}{l}-0.3336 \\
(0.6099)\end{array}$ & $\begin{array}{l}0.4814^{*} \\
(0.2822)\end{array}$ & $\begin{array}{c}-0.9780^{* *} \\
(0.4852)\end{array}$ \\
\hline Middle East & & $\begin{array}{c}0.5731 \\
(0.6334)\end{array}$ & & $\begin{array}{l}-1.6319 \\
(1.2720)\end{array}$ & $\begin{array}{c}0.4783 \\
(0.5255)\end{array}$ & $\begin{array}{c}0.2531 \\
(0.8154)\end{array}$ \\
\hline Decade: 60ies & & $\begin{array}{l}-0.4575 \\
(0.8577)\end{array}$ & & $\begin{array}{l}-0.1640 \\
(1.1435)\end{array}$ & $\begin{array}{l}-0.0479 \\
(0.6999)\end{array}$ & $\begin{array}{c}0.0000 \\
(.)\end{array}$ \\
\hline Decade: 70ies & & $\begin{array}{l}-0.3191 \\
(1.0110)\end{array}$ & & $\begin{array}{l}-0.1270 \\
(1.3252)\end{array}$ & $\begin{array}{c}0.2919 \\
(0.8382)\end{array}$ & $\begin{array}{c}0.3805 \\
(0.9462)\end{array}$ \\
\hline Decade: 80ies & & $\begin{array}{l}-0.4539 \\
(0.8270)\end{array}$ & & $\begin{array}{l}-0.3999 \\
(0.9742)\end{array}$ & $\begin{array}{l}-0.0364 \\
(0.6489)\end{array}$ & $\begin{array}{c}0.4022 \\
(0.6535)\end{array}$ \\
\hline Constant & & $\begin{array}{c}13.0538 * * * \\
(3.7799)\end{array}$ & & $\begin{array}{c}13.9903 * * \\
(5.7093)\end{array}$ & $\begin{array}{c}7.1125^{* * * *} \\
(2.7501)\end{array}$ & $\begin{array}{c}5.5765 \\
(3.5079)\end{array}$ \\
\hline $\mathrm{N}$ & & 1145 & & 1513 & 1677 & 606 \\
\hline Pseudo-R2 & & 0.456 & & 0.594 & 0.387 & 0.282 \\
\hline Log Likelihood & & -92.525 & & -48.334 & -126.963 & -81.446 \\
\hline
\end{tabular}




\section{Appendix VIII: Robustness Checks: Burke and Leigh 2010}

a) all potential reformers

\begin{tabular}{|c|c|c|c|c|}
\hline \multicolumn{5}{|c|}{ Freedom House (FIW, any change) } \\
\hline & $T 3, M 1$ & $T 3, M 2$ & T3, M3 & T3, M4 \\
\hline GDP per capita growth (t-2) & $\begin{array}{c}0.9710^{* * *} \\
(0.0079)\end{array}$ & $\begin{array}{c}0.9705^{* * *} \\
(0.0092)\end{array}$ & $\begin{array}{c}0.9880 \\
(0.0096)\end{array}$ & $\begin{array}{c}0.9949 \\
(0.0108)\end{array}$ \\
\hline $\begin{array}{l}\text { Country-specific development level ( } t- \\
\text { 2) }\end{array}$ & & $\begin{array}{c}1.1882 \\
(0.1727)\end{array}$ & & $\begin{array}{c}0.9912 \\
(0.1488)\end{array}$ \\
\hline Polity score (t-2) & & $\begin{array}{l}0.9106 * * * \\
(0.0182)\end{array}$ & & $\begin{array}{c}1.0247 \\
(0.0160)\end{array}$ \\
\hline Tenure of regime $(\mathrm{t}-1)$ & & $\begin{array}{l}0.9879 * \\
(0.0064)\end{array}$ & & $\begin{array}{c}1.0043 \\
(0.0058)\end{array}$ \\
\hline Democracy in the region $(\mathrm{t}-1)$ & & $\begin{array}{c}1.0060 \\
(0.0069)\end{array}$ & & $\begin{array}{c}1.0014 \\
(0.0076)\end{array}$ \\
\hline $\mathrm{N}$ & 3678 & 3072 & 3577 & 3050 \\
\hline Pseudo-R2 & 0.040 & 0.057 & 0.063 & 0.069 \\
\hline Prob > F & 0.000 & 0.000 & 0.000 & 0.000 \\
\hline
\end{tabular}

\begin{tabular}{|c|c|c|c|c|}
\hline \multicolumn{5}{|c|}{ Freedom House PR, any change) } \\
\hline & $T 3, M 1$ & $T 3, M 2$ & T3, M3 & T3, M4 \\
\hline GDP per capita growth (t-2) & $\begin{array}{c}0.9684 * * * \\
(0.0107)\end{array}$ & $\begin{array}{l}0.9706 * * \\
(0.0116)\end{array}$ & $\begin{array}{c}0.9840 \\
(0.0106)\end{array}$ & $\begin{array}{c}0.9959 \\
(0.0132)\end{array}$ \\
\hline $\begin{array}{l}\text { Country-specific development level (t- } \\
\text { 2) }\end{array}$ & & $\begin{array}{c}1.0645 \\
(0.2420)\end{array}$ & & $\begin{array}{r}1.0352 \\
(0.2120)\end{array}$ \\
\hline Polity score (t-2) & & $\begin{array}{c}0.9213 * * * \\
(0.0201)\end{array}$ & & $\begin{array}{c}1.0201 \\
(0.0209)\end{array}$ \\
\hline Tenure of regime $(\mathrm{t}-1)$ & & $\begin{array}{l}0.9791 * * \\
(0.0100)\end{array}$ & & $\begin{array}{c}1.0088 \\
(0.0087)\end{array}$ \\
\hline Democracy in the region $(\mathrm{t}-1)$ & & $\begin{array}{c}1.0043 \\
(0.0084)\end{array}$ & & $\begin{array}{c}0.9979 \\
(0.0105)\end{array}$ \\
\hline $\mathrm{N}$ & 3146 & 2575 & 2883 & 2425 \\
\hline Pseudo-R2 & 0.040 & 0.055 & 0.067 & 0.062 \\
\hline Prob > F & 0.000 & 0.000 & 0.000 & 0.000 \\
\hline
\end{tabular}

\begin{tabular}{|l|cc|cl|}
\hline \multicolumn{5}{|c|}{ Freedom House (FIW, 1+ points) } \\
\hline & T3, & T3, & & \\
& M1 & M2 & T3, M3 & T3, $\boldsymbol{M 4}$ \\
\hline GDP per capita growth (t-2) & 0.9667 & 0.9699 & $0.9578^{* *}$ & $0.9617^{*}$ \\
& $()$. & $()$. & $(0.0207)$ & $(0.0211)$ \\
Country-specific development level (t-2) & & 1.1469 & & 1.1186 \\
& & $()$. & & $(0.4007)$ \\
Polity score (t-2) & & 0.8983 & & $1.0894^{* *}$ \\
& & $()$. & & $(0.0402)$ \\
Tenure of regime (t-1) & & 0.9667 & & 1.0350 \\
& & $()$. & & $(0.0481)$ \\
Democracy in the region (t-1) & & 1.0011 & & $0.9722^{*}$ \\
& & $()$. & & $(0.0161)$ \\
N & 2354 & 1869 & 1802 & 1488 \\
Pseudo-R2 & 0.100 & 0.139 & 0.154 & 0.185 \\
Prob > F &. &. & 0.000 & 0.000 \\
\hline
\end{tabular}




\begin{tabular}{|c|c|c|c|c|}
\hline \multicolumn{5}{|c|}{ Freedom House (PR, 1+ points) } \\
\hline & $T 3, M 1$ & $T 3, M 2$ & T3, M3 & T3, M4 \\
\hline GDP per capita growth (t-2) & $\begin{array}{c}0.9703 * * * \\
(0.0112)\end{array}$ & $\begin{array}{c}0.9742 * * \\
(0.0122)\end{array}$ & $\begin{array}{l}0.9797 * \\
(0.0114)\end{array}$ & $\begin{array}{c}0.9915 \\
(0.0138)\end{array}$ \\
\hline $\begin{array}{l}\text { Country-specific development level (t- } \\
\text { 2) }\end{array}$ & & $\begin{array}{c}0.9664 \\
(0.2156)\end{array}$ & & $\begin{array}{c}0.9999 \\
(0.1997)\end{array}$ \\
\hline Polity score (t-2) & & $\begin{array}{c}0.9209 * * * \\
(0.0210)\end{array}$ & & $\begin{array}{c}1.0161 \\
(0.0214)\end{array}$ \\
\hline Tenure of regime $(\mathrm{t}-1)$ & & $\begin{array}{l}0.9766 * * \\
(0.0097)\end{array}$ & & $\begin{array}{c}1.0108 \\
(0.0089)\end{array}$ \\
\hline Democracy in the region $(\mathrm{t}-1)$ & & $\begin{array}{c}1.0054 \\
(0.0082)\end{array}$ & & $\begin{array}{c}0.9981 \\
(0.0106)\end{array}$ \\
\hline $\mathrm{N}$ & 3130 & 2561 & 2882 & 2424 \\
\hline Pseudo-R2 & 0.050 & 0.066 & 0.088 & 0.081 \\
\hline Prob > F & 0.000 & 0.000 & 0.000 & 0.000 \\
\hline
\end{tabular}

\begin{tabular}{|l|cc|cc|}
\hline \multicolumn{4}{|c|}{ Gasiorowski (hybrid) } \\
\hline & T3, M1 & T3, M2 & T3, M3 & T3, M4 \\
\hline GDP per capita growth (t-2) & $0.9498^{*}$ & 0.9560 & 0.9817 & 1.0111 \\
& $(0.0263)$ & $(0.0810)$ & $(0.0226)$ & $(0.0450)$ \\
Country-specific development level (t- & & & & \\
2) & & $14.2190^{* * *}$ & & 1.1061 \\
& & $(11.1110)$ & & $(0.7188)$ \\
Polity score (t-2) & & 0.7732 & & $1.3848^{* * *}$ \\
& & $(0.1277)$ & & $(0.1519)$ \\
Tenure of regime (t-1) & & 1.1511 & & 1.2358 \\
& & $(0.1386)$ & & $(0.1675)$ \\
Democracy in the region (t-1) & & $1.0963^{*}$ & & $0.9216^{* * *}$ \\
& & $(0.0523)$ & & $(0.0283)$ \\
N & 410 & 271 & 498 & 413 \\
Pseudo-R2 & 0.286 & 0.461 & 0.241 & 0.455 \\
Prob $>$ F &. &. &. &. \\
\hline
\end{tabular}

\begin{tabular}{|c|c|c|c|c|}
\hline \multicolumn{5}{|c|}{ Gates et al. 2006 (any change) } \\
\hline & T3, M1 & $T 3, M 2$ & T3, M3 & $T 3, M 4$ \\
\hline GDP per capita growth (t-2) & $\begin{array}{l}0.9662 * * \\
(0.0157)\end{array}$ & $\begin{array}{l}0.9610 * * \\
(0.0180)\end{array}$ & $\begin{array}{c}0.9551 * * * \\
(0.0162)\end{array}$ & $\begin{array}{l}0.9553 * * \\
(0.0203)\end{array}$ \\
\hline $\begin{array}{l}\text { Country-specific development level ( } t \text { - } \\
\text { 2) }\end{array}$ & & $\begin{array}{c}1.1450 \\
(0.2706)\end{array}$ & & $\begin{array}{c}0.6982 \\
(0.1917)\end{array}$ \\
\hline Polity score (t-2) & & $\begin{array}{c}0.8078 * * * \\
(0.0281)\end{array}$ & & $\begin{array}{c}1.1448^{* * * *} \\
(0.0459)\end{array}$ \\
\hline Tenure of regime $(\mathrm{t}-1)$ & & $\begin{array}{c}0.9928 \\
(0.0099)\end{array}$ & & $\begin{array}{l}1.0561 * * \\
(0.0272)\end{array}$ \\
\hline Democracy in the region $(\mathrm{t}-1)$ & & $\begin{array}{c}0.9993 \\
(0.0135)\end{array}$ & & $\begin{array}{c}0.9874 \\
(0.0166)\end{array}$ \\
\hline $\mathrm{N}$ & 2587 & 1985 & 1739 & 1460 \\
\hline Pseudo-R2 & 0.075 & 0.166 & 0.105 & 0.161 \\
\hline Prob > F & 0.000 & 0.000 & 0.000 & 0.000 \\
\hline
\end{tabular}




\begin{tabular}{|c|c|c|c|c|}
\hline \multicolumn{5}{|c|}{ Polity IV (any change) } \\
\hline & $T 3, M 1$ & $T 3, M 2$ & T3, M3 & $T 3, M 4$ \\
\hline GDP per capita growth (t-2) & $\begin{array}{c}0.9621 * * * \\
(0.0085)\end{array}$ & $\begin{array}{c}0.9571 * * * \\
(0.0105)\end{array}$ & $\begin{array}{c}0.9783 \\
(0.0131)\end{array}$ & $\begin{array}{c}0.9804 \\
(0.0182)\end{array}$ \\
\hline $\begin{array}{l}\text { Country-specific development level (t- } \\
\text { 2) }\end{array}$ & & $\begin{array}{c}0.8920 \\
(0.1855)\end{array}$ & & $\begin{array}{c}0.7842 \\
(0.1592)\end{array}$ \\
\hline Polity score (t-2) & & $\begin{array}{c}0.7846^{* * *} \\
(0.0267)\end{array}$ & & $\begin{array}{c}1.2088^{* * * *} \\
(0.0484)\end{array}$ \\
\hline Tenure of regime $(\mathrm{t}-1)$ & & $\begin{array}{c}0.9928 \\
(0.0089)\end{array}$ & & $\begin{array}{c}1.0768^{* * *} \\
(0.0286)\end{array}$ \\
\hline Democracy in the region $(\mathrm{t}-1)$ & & $\begin{array}{c}1.0070 \\
(0.0108)\end{array}$ & & $\begin{array}{c}0.9922 \\
(0.0131)\end{array}$ \\
\hline $\mathrm{N}$ & 3752 & 2959 & 3083 & 2598 \\
\hline Pseudo-R2 & 0.072 & 0.163 & 0.070 & 0.150 \\
\hline Prob > F & 0.000 & 0.000 & 0.000 & 0.000 \\
\hline
\end{tabular}

\begin{tabular}{|c|c|c|c|c|}
\hline \multicolumn{5}{|c|}{ Polity IV (3+ points) } \\
\hline & $T 3, M 1$ & $T 3, M 2$ & $T 3, M 3$ & $T 3, M 4$ \\
\hline GDP per capita growth (t-2) & $\begin{array}{c}0.9499 * * * \\
(0.0161)\end{array}$ & $\begin{array}{l}0.9490^{* *} \\
(0.0213)\end{array}$ & $\begin{array}{l}0.9562 * * \\
(0.0195)\end{array}$ & $\begin{array}{l}0.9486^{*} \\
(0.0262)\end{array}$ \\
\hline $\begin{array}{l}\text { Country-specific development level ( } t \text { - } \\
\text { 2) }\end{array}$ & & $\begin{array}{c}0.9404 \\
(0.4043)\end{array}$ & & $\begin{array}{c}0.8387 \\
(0.2650)\end{array}$ \\
\hline Polity score (t-2) & & $\begin{array}{c}0.6191 * * * \\
(0.0469)\end{array}$ & & $\begin{array}{c}1.3767 * * * \\
(0.0651)\end{array}$ \\
\hline Tenure of regime $(t-1)$ & & $\begin{array}{c}1.0003 \\
(0.0209)\end{array}$ & & $\begin{array}{c}1.0554 \\
(0.0377)\end{array}$ \\
\hline Democracy in the region $(\mathrm{t}-1)$ & & $\begin{array}{l}1.0322 * * \\
(0.0158)\end{array}$ & & $\begin{array}{c}0.9606 * * * \\
(0.0138)\end{array}$ \\
\hline $\mathrm{N}$ & 2392 & 1734 & 1757 & 1757 \\
\hline Pseudo-R2 & 0.141 & 0.318 & 0.138 & 0.304 \\
\hline Prob > F & 0.000 & 0.000 & 0.000 & 0.000 \\
\hline
\end{tabular}

\begin{tabular}{|c|c|c|c|c|}
\hline \multicolumn{5}{|c|}{ Polity IV (5+ points) } \\
\hline & T3, M1 & $T 3, M 2$ & T3, M3 & T3, M4 \\
\hline GDP per capita growth (t-2) & $\begin{array}{c}0.9387 * * \\
(0.0246)\end{array}$ & $\begin{array}{l}0.9512^{*} \\
(0.0244)\end{array}$ & $\begin{array}{l}0.9522^{*} \\
(0.0279)\end{array}$ & $\begin{array}{l}0.9312^{*} \\
(0.0376)\end{array}$ \\
\hline $\begin{array}{l}\text { Country-specific development level (t- } \\
\text { 2) }\end{array}$ & & $\begin{array}{c}1.2159 \\
(0.5128)\end{array}$ & & $\begin{array}{c}0.5334 \\
(0.2353)\end{array}$ \\
\hline Polity score $(\mathrm{t}-2)$ & & $\begin{array}{c}0.6435^{* * *} \\
(0.0559)\end{array}$ & & $\begin{array}{c}1.2434 * * * \\
(0.0937)\end{array}$ \\
\hline Tenure of regime $(\mathrm{t}-1)$ & & $\begin{array}{c}0.9884 \\
(0.0232)\end{array}$ & & $\begin{array}{c}1.1903 * * * \\
(0.0615)\end{array}$ \\
\hline Democracy in the region $(\mathrm{t}-1)$ & & $\begin{array}{l}1.0466^{*} \\
(0.0280)\end{array}$ & & $\begin{array}{c}0.9438 * * * \\
(0.0211)\end{array}$ \\
\hline $\mathrm{N}$ & 1632 & 1123 & 1066 & 1066 \\
\hline Pseudo-R2 & 0.232 & 0.351 & 0.249 & 0.349 \\
\hline Prob > F & 0.000 & 0.000 & 0.000 & . \\
\hline
\end{tabular}




\begin{tabular}{|c|c|c|c|c|}
\hline \multicolumn{5}{|c|}{ Reich 2002 (hybrid) } \\
\hline & $T 3, M 1$ & $T 3, M 2$ & T3, M3 & T3, M4 \\
\hline GDP per capita growth $(\mathrm{t}-2)$ & $\begin{array}{c}0.9463^{* *} \\
(0.0261)\end{array}$ & $\begin{array}{l}0.9409 \\
(0.0467)\end{array}$ & $\begin{array}{c}0.9851 \\
(0.0224)\end{array}$ & $\begin{array}{c}1.0422 \\
(0.0399)\end{array}$ \\
\hline Country-specific development level $(\mathrm{t}-2)$ & & $\begin{array}{l}1.1262 \\
(0.3968)\end{array}$ & & $\begin{array}{c}0.8606 \\
(0.4923)\end{array}$ \\
\hline Polity score (t-2) & & $\begin{array}{l}0.8258^{*} \\
(0.0838)\end{array}$ & & $\begin{array}{c}1.3340^{* * *} \\
(0.0767)\end{array}$ \\
\hline Tenure of regime $(\mathrm{t}-1)$ & & $\begin{array}{l}0.9301 \\
(0.0413)\end{array}$ & & $\begin{array}{c}1.0545 \\
(0.0615)\end{array}$ \\
\hline Democracy in the region $(\mathrm{t}-1)$ & & $\begin{array}{l}1.0679 * \\
(0.0398)\end{array}$ & & $\begin{array}{c}0.8905^{* * *} \\
(0.0303)\end{array}$ \\
\hline $\mathrm{N}$ & 706 & 449 & 716 & 619 \\
\hline Pseudo-R2 & 0.265 & 0.352 & 0.202 & 0.375 \\
\hline Prob $>$ F & . & . & . & . \\
\hline
\end{tabular}

\begin{tabular}{|c|c|c|c|c|}
\hline \multicolumn{5}{|c|}{ Skaaning et al. 2015} \\
\hline & $T 3, M 1$ & $T 3, M 2$ & T3, M3 & T3, M4 \\
\hline GDP per capita growth $(\mathrm{t}-2)$ & $\begin{array}{l}0.9751 * \\
(0.0136)\end{array}$ & $\begin{array}{l}0.9704 * * \\
(0.0143)\end{array}$ & $\begin{array}{c}0.9288^{* * *} \\
(0.0191)\end{array}$ & $\begin{array}{c}0.9089 * * * \\
(0.0189)\end{array}$ \\
\hline $\begin{array}{l}\text { Country-specific development level (t- } \\
\text { 2) }\end{array}$ & & $\begin{array}{c}0.9159 \\
(0.2879)\end{array}$ & & $\begin{array}{l}0.6234^{*} \\
(0.1703)\end{array}$ \\
\hline Polity score (t-2) & & $\begin{array}{c}1.0144 \\
(0.0357)\end{array}$ & & $\begin{array}{l}1.0954 * * \\
(0.0430)\end{array}$ \\
\hline Tenure of regime $(\mathrm{t}-1)$ & & $\begin{array}{c}0.9971 \\
(0.0153)\end{array}$ & & $\begin{array}{c}1.0375 \\
(0.0356)\end{array}$ \\
\hline Democracy in the region $(\mathrm{t}-1)$ & & $\begin{array}{l}1.0370 * * \\
(0.0155)\end{array}$ & & $\begin{array}{l}0.9666 * * \\
(0.0144)\end{array}$ \\
\hline $\mathrm{N}$ & 2272 & 1511 & 2021 & 1560 \\
\hline Pseudo-R2 & 0.121 & 0.176 & 0.146 & 0.202 \\
\hline Prob > F & 0.000 & 0.000 & 0.000 & 0.000 \\
\hline
\end{tabular}

\begin{tabular}{|c|c|c|c|c|}
\hline \multicolumn{5}{|c|}{ Wahman et al. 2013 (any change) } \\
\hline & $T 3, M 1$ & $T 3, M 2$ & T3, M3 & T3, M4 \\
\hline GDP per capita growth (t-2) & $\begin{array}{c}0.9673 * * * \\
(0.0072)\end{array}$ & $\begin{array}{c}0.9658 * * * \\
(0.0084)\end{array}$ & $\begin{array}{c}0.9876 \\
(0.0093)\end{array}$ & $\begin{array}{c}0.9892 \\
(0.0119)\end{array}$ \\
\hline Country-specific development level (t- & & 11335 & & 10707 \\
\hline 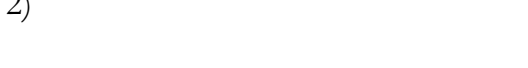 & & $\begin{array}{l}1.1353 \\
(0.1574)\end{array}$ & & $(0.1599)$ \\
\hline Polity score ( $\mathrm{t}-2)$ & & $\begin{array}{c}0.8986^{* * *} \\
(0.0176)\end{array}$ & & $\begin{array}{c}1.0601 * * * \\
(0.0184)\end{array}$ \\
\hline Tenure of regime $(\mathrm{t}-1)$ & & $\begin{array}{c}0.9861 * * \\
(0.0056)\end{array}$ & & $\begin{array}{c}1.0082 \\
(0.0083)\end{array}$ \\
\hline Democracy in the region $(\mathrm{t}-1)$ & & $\begin{array}{c}1.0007 \\
(0.0072)\end{array}$ & & $\begin{array}{c}1.0014 \\
(0.0074)\end{array}$ \\
\hline $\mathrm{N}$ & 3729 & 3123 & 3771 & 3178 \\
\hline Pseudo-R2 & 0.050 & 0.070 & 0.062 & 0.069 \\
\hline Prob > F & 0.000 & 0.000 & 0.000 & 0.000 \\
\hline
\end{tabular}




\begin{tabular}{|c|c|c|c|c|}
\hline \multicolumn{5}{|c|}{ Wahman et al. 2013 (1.5+ points) } \\
\hline & T3, M1 & $T 3, M 2$ & T3, M3 & $T 3, M 4$ \\
\hline GDP per capita growth $(\mathrm{t}-2)$ & $\begin{array}{l}0.9613^{* *} \\
(0.0181)\end{array}$ & $\begin{array}{l}0.9562^{*} \\
(0.0257)\end{array}$ & $\begin{array}{l}0.9551 * * \\
(0.0181)\end{array}$ & $\begin{array}{l}0.9369 * * \\
(0.0264)\end{array}$ \\
\hline $\begin{array}{l}\text { Country-specific development level (t- } \\
\text { 2) }\end{array}$ & & $\begin{array}{c}0.6817 \\
(0.3111)\end{array}$ & & $\begin{array}{c}0.8880 \\
(0.5175)\end{array}$ \\
\hline Polity score (t-2) & & $\begin{array}{c}0.6296 * * * \\
(0.0620)\end{array}$ & & $\begin{array}{c}1.3003 * * * \\
(0.0772)\end{array}$ \\
\hline Tenure of regime $(\mathrm{t}-1)$ & & $\begin{array}{c}0.9596 \\
(0.0241)\end{array}$ & & $\begin{array}{c}1.0748 \\
(0.0528)\end{array}$ \\
\hline Democracy in the region $(t-1)$ & & $\begin{array}{c}1.0272 \\
(0.0257)\end{array}$ & & $\begin{array}{l}0.9416^{* *} \\
(0.0229)\end{array}$ \\
\hline $\mathrm{N}$ & 1823 & 1426 & 1202 & 1058 \\
\hline Pseudo-R2 & 0.147 & 0.392 & 0.145 & 0.292 \\
\hline Prob > F & 0.000 & 0.000 & 0.000 & . \\
\hline
\end{tabular}

\begin{tabular}{|c|c|c|c|c|}
\hline \multicolumn{5}{|c|}{ V-Dem: Electoral Democracy (continuous) } \\
\hline & $T 3, M 1$ & $T 3, M 2$ & T3, M3 & T3, M4 \\
\hline GDP per capita growth $(\mathrm{t}-2)$ & $\begin{array}{l}0.9754^{*} \\
(0.0128)\end{array}$ & $\begin{array}{c}0.9739 * * \\
(0.0131)\end{array}$ & $\begin{array}{c}0.9456 * * * \\
(0.0156)\end{array}$ & $\begin{array}{c}0.9446 * * * \\
(0.0173)\end{array}$ \\
\hline $\begin{array}{l}\text { Country-specific development level ( } t \text { - } \\
\text { 2) }\end{array}$ & & 1.1290 & & 0.9977 \\
\hline & & $(0.1975)$ & & $(0.2517)$ \\
\hline Polity score (t-2) & & 0.9712 & & 1.0016 \\
\hline & & $(0.0203)$ & & $(0.0261)$ \\
\hline Tenure of regime $(\mathrm{t}-1)$ & & $0.9596 * * *$ & & 0.9987 \\
\hline & & $(0.0136)$ & & $(0.0160)$ \\
\hline Democracy in the region $(t-1)$ & & 0.9908 & & 0.9805 \\
\hline & & $(0.0096)$ & & $(0.0158)$ \\
\hline $\mathrm{N}$ & 3574 & 3034 & 2763 & 2311 \\
\hline Pseudo-R2 & 0.093 & 0.113 & 0.085 & 0.093 \\
\hline Prob $>$ F & 0.000 & 0.000 & 0.000 & 0.000 \\
\hline
\end{tabular}

\begin{tabular}{|c|c|c|c|c|}
\hline \multicolumn{5}{|c|}{ V-Dem: Liberal Democracy (continuous) } \\
\hline & $T 3, M 1$ & T3, M2 & T3, M3 & T3, M4 \\
\hline GDP per capita growth $(\mathrm{t}-2)$ & $\begin{array}{c}0.9819 \\
(0.0157)\end{array}$ & $\begin{array}{c}0.9910 \\
(0.0157)\end{array}$ & $\begin{array}{c}0.9567 * * * \\
(0.0155)\end{array}$ & $\begin{array}{c}0.9589 * * \\
(0.0171)\end{array}$ \\
\hline Country-specific development level (t- & & 12225 & & 08262 \\
\hline & & $(0.2451)$ & & $(0.2047)$ \\
\hline Polity score (t-2) & & $\begin{array}{c}0.9814 \\
(0.0204)\end{array}$ & & $\begin{array}{l}1.0442 * \\
(0.0264)\end{array}$ \\
\hline Tenure of regime $(\mathrm{t}-1)$ & & $\begin{array}{c}0.9432 * * * \\
(0.0129)\end{array}$ & & $\begin{array}{c}1.0223 \\
(0.0172)\end{array}$ \\
\hline Democracy in the region $(\mathrm{t}-1)$ & & $\begin{array}{c}0.9866 \\
(0.0088)\end{array}$ & & $\begin{array}{c}0.9816 \\
(0.0165)\end{array}$ \\
\hline $\mathrm{N}$ & 3522 & 3021 & 2532 & 2155 \\
\hline Pseudo-R2 & 0.119 & 0.139 & 0.076 & 0.089 \\
\hline Prob > F & 0.000 & 0.000 & 0.000 & 0.000 \\
\hline
\end{tabular}




\begin{tabular}{|c|c|c|c|c|}
\hline \multicolumn{5}{|c|}{ V-Dem: Participatory Democracy (continuous) } \\
\hline & $T 3, M 1$ & $T 3, M 2$ & T3, M3 & T3, M4 \\
\hline GDP per capita growth $(\mathrm{t}-2)$ & $\begin{array}{c}0.9732 * * \\
(0.0119)\end{array}$ & $\begin{array}{c}0.9815 \\
(0.0120)\end{array}$ & $\begin{array}{c}0.9698^{* *} \\
(0.0151)\end{array}$ & $\begin{array}{c}0.9760 \\
(0.0173)\end{array}$ \\
\hline Country-specific development level (t- & & $14330 * *$ & & 09860 \\
\hline & & $(0.2412)$ & & $(0.1884)$ \\
\hline Polity score (t-2) & & $\begin{array}{c}1.0073 \\
(0.0208)\end{array}$ & & $\begin{array}{c}1.0512 * * \\
(0.0252)\end{array}$ \\
\hline Tenure of regime $(\mathrm{t}-1)$ & & $\begin{array}{c}0.9646^{* * *} \\
(0.0098)\end{array}$ & & $\begin{array}{c}1.0042 \\
(0.0141)\end{array}$ \\
\hline Democracy in the region $(\mathrm{t}-1)$ & & $\begin{array}{c}0.9891 \\
(0.0076)\end{array}$ & & $\begin{array}{c}0.9800 \\
(0.0132)\end{array}$ \\
\hline $\mathrm{N}$ & 4163 & 3645 & 2990 & 2656 \\
\hline Pseudo-R2 & 0.097 & 0.106 & 0.061 & 0.071 \\
\hline Prob > F & 0.000 & 0.000 & 0.000 & 0.000 \\
\hline
\end{tabular}

\begin{tabular}{|c|c|c|c|c|}
\hline \multicolumn{5}{|c|}{ V-Dem: Deliberative Democracy (continuous) } \\
\hline & $T 3, M 1$ & $T 3, M 2$ & T3, M3 & T3, M4 \\
\hline GDP per capita growth $(\mathrm{t}-2)$ & $\begin{array}{c}0.9815 \\
(0.0133)\end{array}$ & $\begin{array}{c}0.9819 \\
(0.0122)\end{array}$ & $\begin{array}{l}0.9633 * * \\
(0.0155)\end{array}$ & $\begin{array}{c}0.9560^{* * *} \\
(0.0164)\end{array}$ \\
\hline $\begin{array}{l}\text { Country-specific development level (t- } \\
\text { 2) }\end{array}$ & & $\begin{array}{l}1.0425 \\
(0.1669)\end{array}$ & & $\begin{array}{c}0.5705^{* *} \\
(0.1314)\end{array}$ \\
\hline Polity score (t-2) & & $\begin{array}{c}1.0042 \\
(0.0203)\end{array}$ & & $\begin{array}{c}1.0243 \\
(0.0277)\end{array}$ \\
\hline Tenure of regime $(t-1)$ & & $\begin{array}{c}0.9739 * * * \\
(0.0099)\end{array}$ & & $\begin{array}{c}1.0213 \\
(0.0145)\end{array}$ \\
\hline Democracy in the region $(\mathrm{t}-1)$ & & $\begin{array}{c}0.9894 \\
(0.0083)\end{array}$ & & $\begin{array}{c}0.9806 \\
(0.0127)\end{array}$ \\
\hline $\mathrm{N}$ & 3886 & 3432 & 2282 & 2134 \\
\hline Pseudo-R2 & 0.095 & 0.095 & 0.066 & 0.080 \\
\hline Prob > F & 0.000 & 0.000 & 0.000 & 0.000 \\
\hline
\end{tabular}

\begin{tabular}{|c|c|c|c|c|}
\hline \multicolumn{5}{|c|}{ V-Dem: Egalitarian Democracy (continuous) } \\
\hline & T3, M1 & $T 3, M 2$ & T3, M3 & T3, M4 \\
\hline GDP per capita growth (t-2) & $\begin{array}{l}0.9758^{*} \\
(0.0137)\end{array}$ & $\begin{array}{c}0.9856 \\
(0.0134)\end{array}$ & $\begin{array}{c}0.9351 * * * \\
(0.0184)\end{array}$ & $\begin{array}{c}0.9379 * * * \\
(0.0213)\end{array}$ \\
\hline $\begin{array}{l}\text { Country-specific development level (t- } \\
\text { 2) }\end{array}$ & & 1.2597 & & 0.9434 \\
\hline & & $(0.2198)$ & & $(0.2246)$ \\
\hline Polity score (t-2) & & $\begin{array}{c}1.0024 \\
(0.0206)\end{array}$ & & $\begin{array}{c}1.0648^{* *} \\
(0.0281)\end{array}$ \\
\hline Tenure of regime $(\mathrm{t}-1)$ & & $\begin{array}{c}0.9466^{* * *} \\
(0.0154)\end{array}$ & & $\begin{array}{c}1.0094 \\
(0.0161)\end{array}$ \\
\hline Democracy in the region $(\mathrm{t}-1)$ & & $\begin{array}{c}0.9972 \\
(0.0088)\end{array}$ & & $\begin{array}{c}0.9830 \\
(0.0159)\end{array}$ \\
\hline $\mathrm{N}$ & 3729 & 3250 & 2500 & 2274 \\
\hline Pseudo-R2 & 0.103 & 0.125 & 0.080 & 0.094 \\
\hline Prob > F & 0.000 & 0.000 & 0.000 & 0.000 \\
\hline
\end{tabular}




\begin{tabular}{|c|c|c|c|c|}
\hline \multicolumn{5}{|c|}{ V-Dem: Electoral Democracy (ordinal) } \\
\hline & T3, M1 & T3, M2 & T3, M3 & T3, M4 \\
\hline GDP per capita growth $(\mathrm{t}-2)$ & $\begin{array}{c}0.9889 \\
(0.0125)\end{array}$ & $\begin{array}{c}0.9821 \\
(0.0123)\end{array}$ & $\begin{array}{c}1.0106 \\
(0.0170)\end{array}$ & $\begin{array}{c}1.0065 \\
(0.0169)\end{array}$ \\
\hline Country-specific development level ( $\mathrm{t}-2)$ & & $\begin{array}{c}1.0520 \\
(0.1662)\end{array}$ & & $\begin{array}{c}0.6699 \\
(0.2570)\end{array}$ \\
\hline Polity score (t-2) & & $\begin{array}{c}0.9753 \\
(0.0214)\end{array}$ & & $\begin{array}{c}1.0012 \\
(0.0593)\end{array}$ \\
\hline Tenure of regime $(\mathrm{t}-1)$ & & $\begin{array}{c}0.9680 * * * \\
(0.0098)\end{array}$ & & $\begin{array}{c}1.0531 \\
(0.0343)\end{array}$ \\
\hline Democracy in the region $(\mathrm{t}-1)$ & & $\begin{array}{c}0.9969 \\
(0.0091)\end{array}$ & & $\begin{array}{c}1.0103 \\
(0.0195)\end{array}$ \\
\hline $\mathrm{N}$ & 3606 & 3047 & 1221 & 1175 \\
\hline Pseudo-R2 & 0.064 & 0.070 & 0.132 & 0.162 \\
\hline Prob $>$ F & 0.000 & 0.000 & 0.000 & . \\
\hline
\end{tabular}

\begin{tabular}{|c|c|c|c|c|}
\hline \multicolumn{5}{|c|}{ V-Dem: Liberal Democracy (ordinal) } \\
\hline & $T 3, M 1$ & $T 3, M 2$ & T3, M3 & $T 3, M 4$ \\
\hline \multirow[t]{2}{*}{ GDP per capita growth $(\mathrm{t}-2)$} & $0.9729 *$ & 0.9767 & 0.9631 & $0.9516^{*}$ \\
\hline & $(0.0152)$ & $(0.0169)$ & $(0.0258)$ & $(0.0255)$ \\
\hline \multirow[t]{2}{*}{ Country-specific development level ( $\mathrm{t}-2)$} & & 1.3803 & & 0.6807 \\
\hline & & $(0.3627)$ & & $(0.1971)$ \\
\hline \multirow[t]{2}{*}{ Polity score (t-2) } & & 1.0150 & & $0.8143^{* * *}$ \\
\hline & & $(0.0294)$ & & $(0.0390)$ \\
\hline \multirow[t]{2}{*}{ Tenure of regime $(\mathrm{t}-1)$} & & $0.9571 * *$ & & $1.0946 * * *$ \\
\hline & & $(0.0173)$ & & $(0.0305)$ \\
\hline \multirow[t]{2}{*}{ Democracy in the region $(t-1)$} & & $0.9795^{*}$ & & 1.0095 \\
\hline & & $(0.0121)$ & & $(0.0164)$ \\
\hline $\mathrm{N}$ & 2817 & 2501 & 1138 & 1108 \\
\hline Pseudo-R2 & 0.136 & 0.156 & 0.148 & 0.232 \\
\hline Prob > F & 0.000 & 0.000 & 0.000 & . \\
\hline
\end{tabular}

\begin{tabular}{|c|c|c|c|c|}
\hline \multicolumn{5}{|c|}{ V-Dem: Participatory Democracy (ordinal) } \\
\hline & $T 3, M 1$ & $T 3, M 2$ & T3, M3 & T3, M4 \\
\hline \multirow[t]{2}{*}{ GDP per capita growth $(\mathrm{t}-2)$} & 0.9834 & 0.9865 & 1.0235 & 1.0162 \\
\hline & $(0.0164)$ & $(0.0171)$ & $(0.0364)$ & $(0.0374)$ \\
\hline \multirow[t]{2}{*}{ Country-specific development level ( $t$-2) } & & 1.1365 & & 0.4804 \\
\hline & & $(0.2476)$ & & $(0.2371)$ \\
\hline \multirow[t]{2}{*}{ Polity score $(\mathrm{t}-2)$} & & 1.0268 & & 0.9293 \\
\hline & & $(0.0276)$ & & $(0.0457)$ \\
\hline \multirow[t]{2}{*}{ Tenure of regime $(t-1)$} & & $0.9545^{* *}$ & & 1.0341 \\
\hline & & $(0.0190)$ & & $(0.0279)$ \\
\hline \multirow[t]{2}{*}{ Democracy in the region $(\mathrm{t}-1)$} & & 0.9906 & & 0.9856 \\
\hline & & $(0.0112)$ & & $(0.0224)$ \\
\hline $\mathrm{N}$ & 3055 & 2717 & 1016 & 1014 \\
\hline Pseudo-R2 & 0.096 & 0.116 & 0.171 & 0.206 \\
\hline Prob > F & 0.000 & 0.000 & . & . \\
\hline
\end{tabular}




\begin{tabular}{|c|c|c|c|c|}
\hline \multicolumn{5}{|c|}{ V-Dem: Deliberative Democracy (ordinal) } \\
\hline & $T 3, M 1$ & $T 3, M 2$ & T3, M3 & T3, M4 \\
\hline GDP per capita growth (t-2) & $\begin{array}{c}0.9889 \\
(0.0168)\end{array}$ & $\begin{array}{c}0.9866 \\
(0.0170)\end{array}$ & $\begin{array}{c}0.9819 \\
(0.0270)\end{array}$ & $\begin{array}{c}0.9680 \\
(0.0269)\end{array}$ \\
\hline Country-specific development level ( $\mathrm{t}-2)$ & & $\begin{array}{c}0.9448 \\
(0.2120)\end{array}$ & & $\begin{array}{r}0.5340^{* *} \\
(0.1356)\end{array}$ \\
\hline Polity score (t-2) & & $\begin{array}{c}1.0488^{*} \\
(0.0274)\end{array}$ & & $\begin{array}{c}1.0150 \\
(0.0962)\end{array}$ \\
\hline Tenure of regime $(\mathrm{t}-1)$ & & $\begin{array}{c}0.9608^{* *} \\
(0.0174)\end{array}$ & & $\begin{array}{c}1.0655^{* *} \\
(0.0340)\end{array}$ \\
\hline Democracy in the region $(\mathrm{t}-1)$ & & $\begin{array}{c}0.9702 * * * \\
(0.0092)\end{array}$ & & $\begin{array}{c}0.9828 \\
(0.0147)\end{array}$ \\
\hline $\mathrm{N}$ & 3117 & 2816 & 1408 & 1407 \\
\hline Pseudo-R2 & 0.117 & 0.130 & 0.144 & 0.173 \\
\hline Prob $>$ F & 0.000 & 0.000 & 0.000 & 0.000 \\
\hline
\end{tabular}

\begin{tabular}{|l|cc|cc|}
\hline \multicolumn{5}{|c|}{ V-Dem: Egalitarian Democracy (ordinal) } \\
\hline & T3, M1 & T3, M2 & T3, M3 & T3, M4 \\
\hline GDP per capita growth (t-2) & 0.9973 & 0.9944 & 0.9782 & 0.9620 \\
Country-specific development level (t-2) & $(0.0174)$ & $(0.0192)$ & $(0.0234)$ & $(0.0230)$ \\
& & 1.1580 & & 0.9761 \\
Polity score (t-2) & & $(0.2985)$ & & $(0.4186)$ \\
& & 0.9799 & & $0.7969 * * *$ \\
Tenure of regime (t-1) & & $0.0334)$ & & $(0.0525)$ \\
& & $0.9600^{* *}$ & & 1.0178 \\
Democracy in the region (t-1) & & $0.9163)$ & & $(0.0329)$ \\
N & & $(0.0106)$ & & $1.0381 *$ \\
Pseudo-R2 & 3184 & 2731 & 1209 & $(0.0198)$ \\
Prob > F & 0.120 & 0.142 & 0.160 & 0.238 \\
\end{tabular}


b) DEM/ AUT only

\begin{tabular}{|l|cc|cc|}
\hline \multicolumn{5}{|c|}{ Freedom House (FIW, any change) } \\
\hline & T3, M1 & T3, M2 & T3, M3 & T3, M4 \\
\hline GDP per capita growth (t-2) & $0.9677^{* * *}$ & $0.9700^{* *}$ & 1.0015 & 1.0106 \\
& $(0.0106)$ & $(0.0124)$ & $(0.0220)$ & $(0.0225)$ \\
Country-specific development level (t- & & 1.3411 & & 1.0319 \\
2) & & $(0.2675)$ & & $(0.2327)$ \\
& & 1.0010 & & $0.9003^{* *}$ \\
Polity score (t-2) & & $(0.0312)$ & & $(0.0454)$ \\
& & 0.9903 & & 1.0320 \\
Tenure of regime (t-1) & & $(0.0094)$ & & $(0.0266)$ \\
& & 1.0051 & & $1.0191 *$ \\
Democracy in the region (t-1) & & $(0.0124)$ & & $(0.0105)$ \\
& 2174 & 1577 & 1539 & 1532 \\
N & 0.060 & 0.068 & 0.107 & 0.125 \\
Pseudo-R2 & 0.000 & 0.000 & 0.000 & 0.000 \\
Prob > F & \multicolumn{3}{|c}{} \\
\hline
\end{tabular}

\begin{tabular}{|l|cc|cc|}
\hline \multicolumn{5}{|c|}{ Freedom House PR, any change) } \\
\hline & $\mathbf{T 3 , \boldsymbol { M } 1}$ & $\mathbf{T 3}, \boldsymbol{M} \mathbf{2}$ & $\mathbf{T 3 , \boldsymbol { M }}$ & $\mathbf{T 3 , \boldsymbol { M } 4}$ \\
\hline GDP per capita growth (t-2) & $0.9625^{* * *}$ & $0.9693^{* *}$ & 0.9826 & 0.9960 \\
& $(0.0132)$ & $(0.0150)$ & $(0.0221)$ & $(0.0243)$ \\
Country-specific development level (t- & & & & \\
2) & & 1.3905 & & 1.0032 \\
& & $(0.3498)$ & & $(0.3057)$ \\
Polity score (t-2) & & 1.0276 & & $0.8670^{*}$ \\
& & $(0.0318)$ & & $(0.0655)$ \\
Tenure of regime (t-1) & & 0.9802 & & $1.1096^{* *}$ \\
& & $(0.0125)$ & & $(0.0449)$ \\
Democracy in the region (t-1) & & $(0.0057$ & & 1.0096 \\
& 2128 & 1562 & 1197 & $(0.0150)$ \\
N & 0.064 & 0.080 & 0.073 & 0.131 \\
Pseudo-R2 & 0.000 & 0.000 & 0.000 & 0.000 \\
Prob > F & & & & \\
\hline
\end{tabular}

\begin{tabular}{|c|c|c|c|c|}
\hline \multicolumn{5}{|c|}{ Freedom House (FIW, 1+ points) } \\
\hline & $\begin{array}{l}\text { T3, } \\
\text { M1 }\end{array}$ & $\begin{array}{l}\text { T3, } \\
\text { M2 }\end{array}$ & T3, M3 & T3, M4 \\
\hline GDP per capita growth (t-2) & $\begin{array}{c}0.9523 \\
(.)\end{array}$ & $\begin{array}{c}0.9496 \\
(.)\end{array}$ & $\begin{array}{l}0.8788^{* * *} \\
(0.0344)\end{array}$ & $\begin{array}{l}0.8351 \text { *** } \\
(0.0421)\end{array}$ \\
\hline Country-specific development level (t-2) & & $\begin{array}{c}1.3026 \\
(.)\end{array}$ & & $\begin{array}{l}0.9670 \\
(0.2992)\end{array}$ \\
\hline Polity score $(\mathrm{t}-2)$ & & $\begin{array}{c}1.0387 \\
(.)\end{array}$ & & $\begin{array}{l}0.8057 * * * \\
(0.0614)\end{array}$ \\
\hline Tenure of regime $(\mathrm{t}-1)$ & & $\begin{array}{c}0.9798 \\
(.)\end{array}$ & & $\begin{array}{l}1.1708^{* * *} \\
(0.0551)\end{array}$ \\
\hline Democracy in the region $(\mathrm{t}-1)$ & & $\begin{array}{c}1.0145 \\
(.)\end{array}$ & & $\begin{array}{l}1.0134 \\
(0.0194)\end{array}$ \\
\hline $\mathrm{N}$ & 1462 & 1053 & 647 & 642 \\
\hline Pseudo-R2 & 0.141 & 0.173 & 0.244 & 0.365 \\
\hline Prob $>$ F & . & . & 0.000 & \\
\hline
\end{tabular}




\begin{tabular}{|l|cc|cc|}
\hline \multicolumn{4}{|c|}{ Freedom House (PR, 1+ points) } \\
\hline & T3, M1 & T3, M2 & T3, M3 & T3, M4 \\
\hline GDP per capita growth (t-2) & $0.9643^{* *}$ & $0.9732^{*}$ & 0.9821 & 0.9964 \\
& $(0.0136)$ & $(0.0156)$ & $(0.0224)$ & $(0.0248)$ \\
Country-specific development level (t-2) & & 1.2926 & & 1.0622 \\
& & $(0.3275)$ & & $(0.3319)$ \\
Polity score (t-2) & & 1.0315 & & $0.8504 * *$ \\
& & $(0.0331)$ & & $(0.0664)$ \\
Tenure of regime (t-1) & & $0.9767 *$ & & $1.1209^{* * *}$ \\
& & $(0.0128)$ & & $(0.0451)$ \\
Democracy in the region (t-1) & & 1.0077 & & 1.0130 \\
& & $(0.0104)$ & & $(0.0157)$ \\
N & 2128 & 1554 & 1194 & 1191 \\
Pseudo-R2 & 0.075 & 0.097 & 0.090 & 0.158 \\
Prob > F & 0.000 & 0.000 & 0.000 & 0.000 \\
\hline
\end{tabular}

\begin{tabular}{|c|c|}
\hline \multicolumn{2}{|c|}{ Gasiorowski (hybrid) } \\
\hline & T3, M3 \\
\hline GDP per capita growth $(\mathrm{t}-2)$ & $\begin{array}{l}0.9458 \\
(0.1527)\end{array}$ \\
\hline Country-specific development level ( $\mathrm{t}-2)$ & \\
\hline Polity score (t-2) & \\
\hline Tenure of regime $(\mathrm{t}-1)$ & \\
\hline Democracy in the region $(\mathrm{t}-1)$ & \\
\hline $\mathrm{N}$ & 95 \\
\hline Pseudo-R2 & \\
\hline Prob > F & \\
\hline
\end{tabular}

\begin{tabular}{|l|cc|cc|}
\hline \multicolumn{5}{|c|}{ Gates et al. 2006 (any change) } \\
\hline & T3, M1 & T3, M2 & T3, M3 & T3, M4 \\
\hline GDP per capita growth (t-2) & 0.9621 & 0.9628 & 0.9814 & 0.9579 \\
& $(0.0227)$ & $(0.0273)$ & $(0.0342)$ & $(0.0386)$ \\
Country-specific development level (t-2) & & 0.8427 & & $0.3052^{* *}$ \\
& & $(0.2902)$ & & $(0.1774)$ \\
Polity score (t-2) & & 0.9680 & & 1.0228 \\
& & $(0.0629)$ & & $(0.1911)$ \\
Tenure of regime (t-1) & & 1.0162 & & $1.3049 * * *$ \\
& & $(0.0132)$ & & $(0.1056)$ \\
Democracy in the region (t-1) & & 1.0136 & & 0.9589 \\
& & $(0.0169)$ & & $(0.0282)$ \\
N & 1687 & 1121 & 658 & 658 \\
Pseudo-R2 & 0.163 & 0.171 & 0.253 & 0.446 \\
Prob > F & 0.000 & 0.000 & 0.000 &. \\
\hline
\end{tabular}




\begin{tabular}{|c|c|c|c|c|}
\hline \multicolumn{5}{|c|}{ Polity IV (any change) } \\
\hline & $T 3, M 1$ & $T 3, M 2$ & T3, M3 & $T 3, M 4$ \\
\hline GDP per capita growth $(\mathrm{t}-2)$ & $\begin{array}{c}0.9468^{* * *} * \\
(0.0131)\end{array}$ & $\begin{array}{c}0.9587 * * \\
(0.0163)\end{array}$ & $\begin{array}{c}0.9913 \\
(0.0162)\end{array}$ & $\begin{array}{c}0.9895 \\
(0.0226)\end{array}$ \\
\hline Country-specific development level (t- & & 08623 & & $06652 *$ \\
\hline & & $(0.3049)$ & & $(0.1603)$ \\
\hline Polity score (t-2) & & $\begin{array}{c}0.6535^{* * *} \\
(0.0656)\end{array}$ & & $\begin{array}{c}1.0662 \\
(0.1055)\end{array}$ \\
\hline Tenure of regime $(\mathrm{t}-1)$ & & $\begin{array}{c}1.0092 \\
(0.0176)\end{array}$ & & $\begin{array}{c}1.1796^{* * *} \\
(0.0461)\end{array}$ \\
\hline Democracy in the region $(\mathrm{t}-1)$ & & $\begin{array}{c}1.0662^{* * *} \\
(0.0177)\end{array}$ & & $\begin{array}{c}0.9836 \\
(0.0131)\end{array}$ \\
\hline $\mathrm{N}$ & 2149 & 1358 & 1360 & 1360 \\
\hline Pseudo-R2 & 0.167 & 0.247 & 0.106 & 0.188 \\
\hline Prob > F & 0.000 & 0.000 & 0.000 & 0.000 \\
\hline
\end{tabular}

\begin{tabular}{|c|c|c|c|c|}
\hline \multicolumn{5}{|c|}{ Polity IV (3+ points) } \\
\hline & $T 3, M 1$ & $T 3, M 2$ & T3, M3 & $T 3, M 4$ \\
\hline GDP per capita growth (t-2) & $\begin{array}{l}0.9406^{* * *} \\
(0.0195)\end{array}$ & $\begin{array}{l}0.9497 * \\
(0.0265)\end{array}$ & $\begin{array}{c}0.9550 \\
(0.0283)\end{array}$ & $\begin{array}{l}0.9319 * * \\
(0.0317)\end{array}$ \\
\hline $\begin{array}{l}\text { Country-specific development level (t- } \\
\text { 2) }\end{array}$ & & $\begin{array}{c}1.1757 \\
(0.5365)\end{array}$ & & $\begin{array}{l}0.3641 * \\
(0.2022)\end{array}$ \\
\hline Polity score (t-2) & & $\begin{array}{c}0.8951 \\
(0.1125)\end{array}$ & & $\begin{array}{c}0.9568 \\
(0.1656)\end{array}$ \\
\hline Tenure of regime $(\mathrm{t}-1)$ & & $\begin{array}{l}1.0757 * * \\
(0.0355)\end{array}$ & & $\begin{array}{c}1.3221 * * * \\
(0.0826)\end{array}$ \\
\hline Democracy in the region $(\mathrm{t}-1)$ & & $\begin{array}{c}1.0615^{* * *} \\
(0.0216)\end{array}$ & & $\begin{array}{c}0.9317 * * * \\
(0.0238)\end{array}$ \\
\hline $\mathrm{N}$ & 1732 & 1118 & 834 & 834 \\
\hline Pseudo-R2 & 0.258 & 0.315 & 0.210 & 0.352 \\
\hline Prob $>$ F & 0.000 & 0.000 & . & . \\
\hline
\end{tabular}

\begin{tabular}{|c|c|c|c|c|}
\hline \multicolumn{5}{|c|}{ Polity IV (5+ points) } \\
\hline & $T 3, M 1$ & $T 3, M 2$ & $T 3, M 3$ & $T 3, M 4$ \\
\hline GDP per capita growth (t-2) & $\begin{array}{c}0.9380^{* * *} \\
(0.0217)\end{array}$ & $\begin{array}{l}0.9491 * \\
(0.0277)\end{array}$ & $\begin{array}{l}0.9494 * \\
(0.0295)\end{array}$ & $\begin{array}{c}0.9211 * * \\
(0.0338)\end{array}$ \\
\hline Country-specific development level ( $t$ - & & 08872 & & $04264 *$ \\
\hline & & $(0.4300)$ & & $(0.2092)$ \\
\hline Polity score (t-2) & & $\begin{array}{c}0.9762 \\
(0.1412)\end{array}$ & & $\begin{array}{c}1.0605 \\
(0.1883)\end{array}$ \\
\hline Tenure of regime $(\mathrm{t}-1)$ & & $\begin{array}{c}1.0429 \\
(0.0469)\end{array}$ & & $\begin{array}{c}1.2871 * * * \\
(0.0874)\end{array}$ \\
\hline Democracy in the region $(\mathrm{t}-1)$ & & $\begin{array}{c}1.0662^{* *} \\
(0.0335)\end{array}$ & & $\begin{array}{c}0.9220^{* * *} \\
(0.0269)\end{array}$ \\
\hline $\mathrm{N}$ & 1336 & 873 & 774 & 774 \\
\hline Pseudo-R2 & 0.298 & 0.334 & 0.257 & 0.390 \\
\hline Prob > F & 0.000 & . & . & . \\
\hline
\end{tabular}




\begin{tabular}{|c|c|c|c|c|}
\hline \multicolumn{5}{|c|}{ Reich 2002 (hybrid) } \\
\hline & $T 3, M 1$ & $T 3, M 2$ & T3, M3 & T3, M4 \\
\hline $\begin{array}{l}\text { GDP per capita growth (t-2) } \\
\text { Country-specific development level (t- } \\
\text { 2) }\end{array}$ & $\begin{array}{c}\text { no } \\
\text { observations }\end{array}$ & $\begin{array}{c}\text { no } \\
\text { observations }\end{array}$ & $\begin{array}{c}\text { not } \\
\text { concave }\end{array}$ & $\begin{array}{c}\text { not } \\
\text { concave }\end{array}$ \\
\hline Polity score (t-2) & & & & \\
\hline Tenure of regime $(\mathrm{t}-1)$ & & & & \\
\hline Democracy in the region $(t-1)$ & & & & \\
\hline $\begin{array}{l}\text { N } \\
\text { Pseudo-R2 } \\
\text { Prob }>\text { F }\end{array}$ & & & & \\
\hline
\end{tabular}

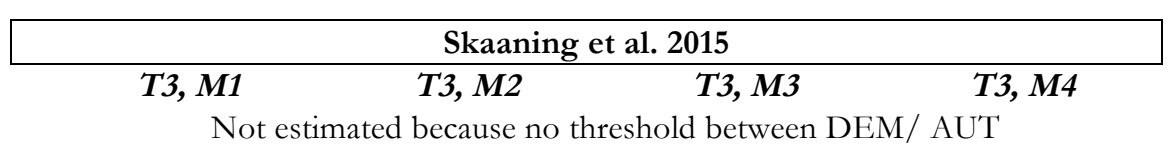

\begin{tabular}{|c|c|c|c|c|}
\hline \multicolumn{5}{|c|}{ Wahman et al. 2013 (any change) } \\
\hline & $T 3, M 1$ & $T 3, M 2$ & T3, M3 & $T 3, M 4$ \\
\hline GDP per capita growth $(\mathrm{t}-2)$ & $\begin{array}{c}0.9625^{* * *} * \\
(0.0094)\end{array}$ & $\begin{array}{c}0.9643^{* * *} \\
(0.0109)\end{array}$ & $\begin{array}{c}1.0022 \\
(0.0216)\end{array}$ & $\begin{array}{c}1.0056 \\
(0.0219)\end{array}$ \\
\hline Country-specific development level (t- & & & & \\
\hline 2) & & $\begin{array}{c}1.1286 \\
(0.1928)\end{array}$ & & $\begin{array}{c}1.1130 \\
(0.2314)\end{array}$ \\
\hline Polity score (t-2) & & $\begin{array}{c}0.9369 * * \\
(0.0304)\end{array}$ & & $\begin{array}{c}0.8606 \\
(0.0936)\end{array}$ \\
\hline Tenure of regime $(t-1)$ & & $\begin{array}{c}0.9948 \\
(0.0081)\end{array}$ & & $\begin{array}{c}1.0956^{* * *} \\
(0.0323)\end{array}$ \\
\hline Democracy in the region $(\mathrm{t}-1)$ & & $\begin{array}{c}1.0040 \\
(0.0109)\end{array}$ & & $\begin{array}{c}1.0139 \\
(0.0108)\end{array}$ \\
\hline $\mathrm{N}$ & 2402 & 1792 & 1383 & 1383 \\
\hline Pseudo-R2 & 0.080 & 0.086 & 0.119 & 0.138 \\
\hline Prob > F & 0.000 & 0.000 & 0.000 & 0.000 \\
\hline
\end{tabular}




\begin{tabular}{|l|cc|cc|}
\hline \multicolumn{4}{|c|}{ Wahman et al. 2013 (1.5+ points) } \\
\hline & & & T3, & \\
& T3, M1 & T3, M2 & M3 & T3, M4 \\
\hline GDP per capita growth (t-2) & $0.9507^{* *}$ & $0.9531^{*}$ & 0.9654 & not \\
& $(0.0232)$ & $(0.0277)$ & $()$. & concave \\
Country-specific development level (t- & & & & \\
2) & & 0.6352 & & \\
& & $(0.3304)$ & & \\
Polity score (t-2) & & $0.7003^{* * *}$ & & \\
& & $(0.0765)$ & & \\
Tenure of regime (t-1) & & 0.9688 & & \\
& & $(0.0249)$ & & \\
Democracy in the region (t-1) & & $(0.0241$ & & \\
& 1325 & 980 & 230 & \\
N & 0.191 & 0.308 & 0.393 & \\
Pseudo-R2 & 0.000 & 0.000 & . \\
Prob > F & \multicolumn{3}{|c}{} \\
\hline
\end{tabular}




\section{Appendix IX: Robustness Checks: Cornell 2013}

a) all potential reformers

\begin{tabular}{|c|c|c|c|c|}
\hline & $\begin{array}{c}\text { Freedom } \\
\text { House } \\
\text { (FIW, any } \\
\text { change) }\end{array}$ & $\begin{array}{c}\text { Freedom } \\
\text { House (PR, } \\
\text { any change) }\end{array}$ & $\begin{array}{l}\text { Freedom } \\
\text { House } \\
\text { (FIW: } \\
\text { 1+points) }\end{array}$ & $\begin{array}{c}\text { Freedom } \\
\text { House (PR: } \\
\text { 1+points) }\end{array}$ \\
\hline Democracy aid & $\begin{array}{c}61.1950 * * * \\
(15.1009)\end{array}$ & $\begin{array}{c}20.6502 \\
(18.4736)\end{array}$ & $\begin{array}{c}57.9976 * * * \\
(9.1796)\end{array}$ & $\begin{array}{c}21.9191 \\
(18.9972)\end{array}$ \\
\hline Other aid & $\begin{array}{c}0.1495 \\
(0.1477)\end{array}$ & $\begin{array}{l}-0.0973 \\
(0.1269)\end{array}$ & $\begin{array}{l}-0.0128 \\
(0.1365)\end{array}$ & $\begin{array}{l}-0.1532 \\
(0.1622)\end{array}$ \\
\hline GDP per capita & $\begin{array}{c}0.0085 \\
(0.0064)\end{array}$ & $\begin{array}{c}0.0069 \\
(0.0055)\end{array}$ & $\begin{array}{c}0.0038 \\
(0.0054)\end{array}$ & $\begin{array}{c}0.0059 \\
(0.0065)\end{array}$ \\
\hline Trade openness & $\begin{array}{l}-0.0000 \\
(0.0002)\end{array}$ & $\begin{array}{c}0.0000 \\
(0.0001)\end{array}$ & $\begin{array}{c}0.0001 \\
(0.0001)\end{array}$ & $\begin{array}{c}0.0001 \\
(0.0002)\end{array}$ \\
\hline Economic growth & $\begin{array}{c}-0.0046^{* * *} \\
(0.0011)\end{array}$ & $\begin{array}{c}-0.0030^{* * *} \\
(0.0010)\end{array}$ & $\begin{array}{c}-0.0021 * * * \\
(0.0008)\end{array}$ & $\begin{array}{c}-0.0030 * * * \\
(0.0011)\end{array}$ \\
\hline Religious fractionalization & $\begin{array}{c}0.0267 \\
(0.0428)\end{array}$ & $\begin{array}{l}-0.0003 \\
(0.0451)\end{array}$ & $\begin{array}{l}-0.0031 \\
(0.0252)\end{array}$ & $\begin{array}{l}-0.0086 \\
(0.0487)\end{array}$ \\
\hline Ethnic fractionalization & $\begin{array}{c}0.0367 \\
(0.0263)\end{array}$ & $\begin{array}{c}0.0439 \\
(0.0347)\end{array}$ & $\begin{array}{c}0.0383 \\
(0.0256)\end{array}$ & $\begin{array}{c}0.0587 \\
(0.0412)\end{array}$ \\
\hline Monarchy & $\begin{array}{c}0.0591 \\
(0.0597)\end{array}$ & $\begin{array}{c}0.0557 \\
(0.0459)\end{array}$ & $\begin{array}{l}-0.0041 \\
(0.0301)\end{array}$ & $\begin{array}{c}0.0593 \\
(0.0501)\end{array}$ \\
\hline Military regime & $\begin{array}{c}0.1217 * * * \\
(0.0453)\end{array}$ & $\begin{array}{c}0.0958 * * \\
(0.0388)\end{array}$ & $\begin{array}{l}0.0501 * \\
(0.0273)\end{array}$ & $\begin{array}{c}0.0955 * * \\
(0.0404)\end{array}$ \\
\hline Limited multi-party regime & $\begin{array}{c}0.0600 \\
(0.0412)\end{array}$ & $\begin{array}{c}0.0554 \\
(0.0376)\end{array}$ & $\begin{array}{l}-0.0021 \\
(0.0268)\end{array}$ & $\begin{array}{c}0.0548 \\
(0.0425)\end{array}$ \\
\hline Other regimes & $\begin{array}{c}0.1652 * * * \\
(0.0567)\end{array}$ & $\begin{array}{c}0.1568 * * * \\
(0.0483)\end{array}$ & $\begin{array}{c}0.0912 * * \\
(0.0365)\end{array}$ & $\begin{array}{c}0.1539 * * * \\
(0.0495)\end{array}$ \\
\hline Democracy & $\begin{array}{l}-0.0358 \\
(0.0530)\end{array}$ & $\begin{array}{l}-0.0401 \\
(0.0456)\end{array}$ & $\begin{array}{c}-0.0558^{*} \\
(0.0308)\end{array}$ & $\begin{array}{c}0.0564 \\
(0.0663)\end{array}$ \\
\hline Monarchy $*$ democracy aid & $\begin{array}{c}-66.0467 * * * \\
(15.6662)\end{array}$ & $\begin{array}{l}-25.0233 \\
(18.7889)\end{array}$ & $\begin{array}{c}-61.2776^{* * *} \\
(9.8595)\end{array}$ & $\begin{array}{l}-25.5857 \\
(19.1735)\end{array}$ \\
\hline Military regime $*$ democracy aid & $\begin{array}{c}-62.9894 * * * \\
(17.8179)\end{array}$ & $\begin{array}{l}-18.6355 \\
(21.4002)\end{array}$ & $\begin{array}{c}-62.0312^{* * *} \\
(9.3744)\end{array}$ & $\begin{array}{l}-19.7996 \\
(21.6946)\end{array}$ \\
\hline Limited multi-party regime $*$ democracy aid & $\begin{array}{c}-61.1811 * * * \\
(15.0922)\end{array}$ & $\begin{array}{l}-20.7037 \\
(18.5144)\end{array}$ & $\begin{array}{c}-58.3444^{* * *} \\
(9.2185)\end{array}$ & $\begin{array}{l}-21.9384 \\
(19.0116)\end{array}$ \\
\hline Other regimes $*$ democracy aid & $\begin{array}{c}-44.9823^{* * *} \\
(15.6906)\end{array}$ & $\begin{array}{c}-2.3659 \\
(18.6366)\end{array}$ & $\begin{array}{c}-58.9861 * * * \\
(10.2038)\end{array}$ & $\begin{array}{c}-3.5342 \\
(18.9767)\end{array}$ \\
\hline Democracy $*$ democracy aid & $\begin{array}{c}-62.2346^{* * *} \\
(15.0788)\end{array}$ & $\begin{array}{l}-20.5991 \\
(18.4754)\end{array}$ & $\begin{array}{c}-57.7519 * * * \\
(9.4681)\end{array}$ & $\begin{array}{l}-23.6436 \\
(19.0114)\end{array}$ \\
\hline Constant & $\begin{array}{c}0.0888 \\
(0.0803)\end{array}$ & $\begin{array}{c}0.0998 \\
(0.0704)\end{array}$ & $\begin{array}{c}0.1350 * * \\
(0.0571)\end{array}$ & $\begin{array}{l}0.1723 * \\
(0.0939)\end{array}$ \\
\hline $\mathrm{N}$ & 1915 & 1775 & 1638 & 1419 \\
\hline $\mathrm{R} 2$ & 0.053 & 0.053 & 0.055 & 0.049 \\
\hline Prob $>$ F & 0.000 & 0.000 & 0.000 & 0.000 \\
\hline
\end{tabular}




\begin{tabular}{|c|c|c|c|c|c|}
\hline & $\begin{array}{c}\text { Gasiorowski } \\
\text { (hybrid) }\end{array}$ & $\begin{array}{c}\text { Gates et al. } \\
2006 \text { (any } \\
\text { change) }\end{array}$ & $\begin{array}{l}\text { Polity IV } \\
\text { (any } \\
\text { change) }\end{array}$ & $\begin{array}{c}\text { Polity IV } \\
\text { (3+ points) }\end{array}$ & $\begin{array}{c}\text { Polity IV } \\
(5+\text { points })\end{array}$ \\
\hline Democracy aid & $\begin{array}{c}121.9018 \\
(.)\end{array}$ & $\begin{array}{c}32.4767 \\
(24.7706)\end{array}$ & $\begin{array}{c}46.2489 * * \\
(19.8624)\end{array}$ & $\begin{array}{l}33.9180^{*} \\
(17.4925)\end{array}$ & $\begin{array}{c}42.5880^{* *} \\
(17.2859)\end{array}$ \\
\hline Other aid & $\begin{array}{c}-0.3117 \\
(.)\end{array}$ & $\begin{array}{l}-0.0824 \\
(0.1462)\end{array}$ & $\begin{array}{c}0.1246 \\
(0.1584)\end{array}$ & $\begin{array}{c}0.0894 \\
(0.1623)\end{array}$ & $\begin{array}{c}0.0669 \\
(0.2078)\end{array}$ \\
\hline Trade openness & $\begin{array}{c}0.0007 \\
(.)\end{array}$ & $\begin{array}{c}0.0001 \\
(0.0002)\end{array}$ & $\begin{array}{l}-0.0001 \\
(0.0001)\end{array}$ & $\begin{array}{c}-0.0002 * * \\
(0.0001)\end{array}$ & $\begin{array}{l}-0.0000 \\
(0.0001)\end{array}$ \\
\hline Economic growth & $\begin{array}{c}0.0008 \\
(.)\end{array}$ & $\begin{array}{c}-0.0023 * * \\
(0.0011)\end{array}$ & $\begin{array}{c}-0.0024^{*} \\
(0.0012)\end{array}$ & $\begin{array}{l}-0.0013 \\
(0.0009)\end{array}$ & $\begin{array}{l}-0.0010 \\
(0.0008)\end{array}$ \\
\hline Religious fractionalization & $\begin{array}{c}0.1461 \\
(.)\end{array}$ & $\begin{array}{l}-0.0151 \\
(0.0316)\end{array}$ & $\begin{array}{l}-0.0102 \\
(0.0375)\end{array}$ & $\begin{array}{l}-0.0266 \\
(0.0296)\end{array}$ & $\begin{array}{l}-0.0412 \\
(0.0334)\end{array}$ \\
\hline Monarchy & $\begin{array}{c}-0.0979 \\
(.)\end{array}$ & $\begin{array}{c}-0.0933 * * \\
(0.0435)\end{array}$ & $\begin{array}{l}-0.0100 \\
(0.0545)\end{array}$ & $\begin{array}{l}-0.0115 \\
(0.0303)\end{array}$ & $\begin{array}{c}0.0305 \\
(0.0368)\end{array}$ \\
\hline Military regime & $\begin{array}{c}0.0724 \\
(.)\end{array}$ & $\begin{array}{c}0.0304 \\
(0.0423)\end{array}$ & $\begin{array}{c}0.0692 \\
(0.0432)\end{array}$ & $\begin{array}{c}0.0695 * * \\
(0.0321)\end{array}$ & $\begin{array}{c}0.0725 * * \\
(0.0349)\end{array}$ \\
\hline Limited multi-party regime & $\begin{array}{c}0.0383 \\
(.)\end{array}$ & $\begin{array}{l}-0.0316 \\
(0.0384)\end{array}$ & $\begin{array}{l}-0.0410 \\
(0.0425)\end{array}$ & $\begin{array}{l}-0.0077 \\
(0.0270)\end{array}$ & $\begin{array}{c}0.0262 \\
(0.0286)\end{array}$ \\
\hline Other regimes & $\begin{array}{c}0.3391 \\
(.)\end{array}$ & $\begin{array}{c}0.1481 \\
(0.0927)\end{array}$ & $\begin{array}{c}0.0001 \\
(0.0573)\end{array}$ & $\begin{array}{l}-0.0065 \\
(0.0400)\end{array}$ & $\begin{array}{c}0.0295 \\
(0.0454)\end{array}$ \\
\hline Democracy & $\begin{array}{c}-0.0282 \\
(.)\end{array}$ & $\begin{array}{c}-0.1000^{* *} \\
(0.0418)\end{array}$ & $\begin{array}{c}-0.1199 * * \\
(0.0489)\end{array}$ & $\begin{array}{c}-0.0770^{* *} \\
(0.0340)\end{array}$ & $\begin{array}{l}-0.0197 \\
(0.0505)\end{array}$ \\
\hline Monarchy $*$ democracy aid & $\begin{array}{c}-190.8728 \\
(.)\end{array}$ & $\begin{array}{l}-32.0736 \\
(24.8162)\end{array}$ & $\begin{array}{c}-49.7729 * * \\
(21.6189)\end{array}$ & $\begin{array}{c}-32.6033^{*} \\
(18.9991)\end{array}$ & $\begin{array}{c}-43.1492^{* *} \\
(18.8325)\end{array}$ \\
\hline Democracy $*$ democracy aid & $\begin{array}{c}-121.1696 \\
(.)\end{array}$ & $\begin{array}{l}-34.5063 \\
(24.4170)\end{array}$ & $\begin{array}{c}-46.2308^{* *} \\
(19.8112)\end{array}$ & $\begin{array}{c}-34.7763 * * \\
(17.4695)\end{array}$ & $\begin{array}{c}-43.5461 * * \\
(17.2398)\end{array}$ \\
\hline Constant & $\begin{array}{c}-0.1181 \\
(.)\end{array}$ & $\begin{array}{c}0.1546 * * * \\
(0.0555)\end{array}$ & $\begin{array}{c}0.2044 * * * \\
(0.0638)\end{array}$ & $\begin{array}{c}0.1872 * * * \\
(0.0601)\end{array}$ & $\begin{array}{c}0.1113 \\
(0.0705)\end{array}$ \\
\hline $\mathrm{N}$ & 161 & 901 & 1709 & 1368 & 1089 \\
\hline $\mathrm{R} 2$ & 0.173 & 0.060 & 0.078 & 0.046 & 0.035 \\
\hline Prob $>$ F & . & 0.000 & 0.000 & 0.000 & 0.000 \\
\hline
\end{tabular}




\begin{tabular}{|c|c|c|c|c|}
\hline & $\begin{array}{c}\text { Reich } 2002 \\
\text { (hybrid) }\end{array}$ & $\begin{array}{l}\text { Skaaning et } \\
\text { al. } 2015\end{array}$ & $\begin{array}{l}\text { Wahman et } \\
\text { al. } 2013 \text { (any } \\
\text { change) }\end{array}$ & $\begin{array}{l}\text { Wahman et } \\
\text { al. } 2013 \\
(1.5+\text { points })\end{array}$ \\
\hline Democracy aid & $\begin{array}{c}35.4970 \\
(36.8065)\end{array}$ & $\begin{array}{c}32.0170 \\
(24.5775)\end{array}$ & $\begin{array}{c}67.5590^{* * *} \\
(16.3281)\end{array}$ & $\begin{array}{c}53.7257 * * * \\
(9.0427)\end{array}$ \\
\hline Other aid & $\begin{array}{c}0.1637 \\
(0.1932)\end{array}$ & $\begin{array}{c}0.0188 \\
(0.1638)\end{array}$ & $\begin{array}{c}0.0651 \\
(0.1499)\end{array}$ & $\begin{array}{c}0.0350 \\
(0.0869)\end{array}$ \\
\hline GDP per capita & $\begin{array}{l}-0.0019 \\
(0.0054)\end{array}$ & $\begin{array}{l}-0.0028 \\
(0.0051)\end{array}$ & $\begin{array}{c}0.0087 \\
(0.0071)\end{array}$ & $\begin{array}{c}0.0012 \\
(0.0024)\end{array}$ \\
\hline Trade openness & $\begin{array}{c}-0.0003 * \\
(0.0002)\end{array}$ & $\begin{array}{l}-0.0001 \\
(0.0001)\end{array}$ & $\begin{array}{l}-0.0000 \\
(0.0002)\end{array}$ & $\begin{array}{l}-0.0000 \\
(0.0001)\end{array}$ \\
\hline Economic growth & $\begin{array}{c}0.0012 \\
(0.0011)\end{array}$ & $\begin{array}{c}0.0005 \\
(0.0008)\end{array}$ & $\begin{array}{c}-0.0054 * * * \\
(0.0013)\end{array}$ & $\begin{array}{c}-0.0015^{* *} \\
(0.0007)\end{array}$ \\
\hline Religious fractionalization & $\begin{array}{c}0.1441 * * * \\
(0.0508)\end{array}$ & $\begin{array}{l}-0.0142 \\
(0.0369)\end{array}$ & $\begin{array}{c}0.0255 \\
(0.0534)\end{array}$ & $\begin{array}{c}0.0024 \\
(0.0266)\end{array}$ \\
\hline Ethnic fractionalization & $\begin{array}{l}-0.1354 \\
(0.0854)\end{array}$ & $\begin{array}{l}-0.0269 \\
(0.0350)\end{array}$ & $\begin{array}{c}0.0443 \\
(0.0308)\end{array}$ & $\begin{array}{c}0.0019 \\
(0.0182)\end{array}$ \\
\hline Monarchy & $\begin{array}{c}0.1501 * * \\
(0.0697)\end{array}$ & $\begin{array}{l}-0.0195 \\
(0.0515)\end{array}$ & $\begin{array}{c}0.0436 \\
(0.0698)\end{array}$ & $\begin{array}{c}0.0054 \\
(0.0239)\end{array}$ \\
\hline Military regime & $\begin{array}{c}0.0517 \\
(0.0383)\end{array}$ & $\begin{array}{c}0.0561 \\
(0.0436)\end{array}$ & $\begin{array}{c}0.1567 * * * \\
(0.0474)\end{array}$ & $\begin{array}{c}0.0606 * * \\
(0.0273)\end{array}$ \\
\hline Limited multi-party regime & $\begin{array}{c}0.0802^{* *} \\
(0.0344)\end{array}$ & $\begin{array}{l}-0.0096 \\
(0.0416)\end{array}$ & $\begin{array}{c}0.0580 \\
(0.0473)\end{array}$ & $\begin{array}{c}0.0075 \\
(0.0222)\end{array}$ \\
\hline Other regimes & $\begin{array}{c}0.2298^{* * *} \\
(0.0752)\end{array}$ & $\begin{array}{c}0.0577 \\
(0.0516)\end{array}$ & $\begin{array}{c}0.1295^{* *} \\
(0.0590)\end{array}$ & $\begin{array}{c}0.0656 * * \\
(0.0333)\end{array}$ \\
\hline Democracy & $\begin{array}{c}0.0122 \\
(0.0302)\end{array}$ & $\begin{array}{c}0.0000 \\
(.)\end{array}$ & $\begin{array}{l}-0.0529 \\
(0.0605)\end{array}$ & $\begin{array}{l}-0.0289 \\
(0.0266)\end{array}$ \\
\hline Monarchy * democracy aid & $\begin{array}{l}-43.2982 \\
(36.0790)\end{array}$ & $\begin{array}{l}-38.8568 \\
(23.6331)\end{array}$ & $\begin{array}{c}-72.1734^{* * *} \\
(17.5600)\end{array}$ & $\begin{array}{c}-55.3920^{* * *} \\
(9.0603)\end{array}$ \\
\hline Military regime $*$ democracy aid & $\begin{array}{l}-35.2628 \\
(36.5699)\end{array}$ & $\begin{array}{l}-22.5489 \\
(25.9439)\end{array}$ & $\begin{array}{c}-58.1747 * * * \\
(19.2091)\end{array}$ & $\begin{array}{c}-56.8549 * * * \\
(10.4651)\end{array}$ \\
\hline Limited multi-party regime $*$ democracy aid & $\begin{array}{l}-36.9539 \\
(37.5993)\end{array}$ & $\begin{array}{l}-32.3468 \\
(24.4883)\end{array}$ & $\begin{array}{c}-65.9406^{* * *} \\
(16.4120)\end{array}$ & $\begin{array}{c}-54.0792^{* * *} \\
(9.0517)\end{array}$ \\
\hline Other regimes $*$ democracy aid & $\begin{array}{c}-133.3476^{*} \\
(73.3240)\end{array}$ & $\begin{array}{l}-15.9485 \\
(24.3394)\end{array}$ & $\begin{array}{c}-49.3876^{* * *} \\
(17.1713)\end{array}$ & $\begin{array}{c}-53.1112^{* * * *} \\
(9.8640)\end{array}$ \\
\hline Democracy $*$ democracy aid & $\begin{array}{l}-37.7711 \\
(36.4500)\end{array}$ & $\begin{array}{c}0.0000 \\
(.)\end{array}$ & $\begin{array}{c}-68.5522 * * * \\
(16.3272)\end{array}$ & $\begin{array}{c}-53.4936 * * * \\
(9.0448)\end{array}$ \\
\hline Constant & $\begin{array}{c}0.0182 \\
(0.0997)\end{array}$ & $\begin{array}{c}0.2553 * * \\
(0.1065)\end{array}$ & $\begin{array}{c}0.1053 \\
(0.0847)\end{array}$ & $\begin{array}{c}0.0900^{* *} \\
(0.0457)\end{array}$ \\
\hline $\mathrm{N}$ & 502 & 1008 & 1921 & 1766 \\
\hline $\mathrm{R} 2$ & 0.078 & 0.070 & 0.072 & 0.058 \\
\hline Prob $>$ F & 0.000 & 0.000 & 0.000 & 0.000 \\
\hline
\end{tabular}


V-Dem continuous:

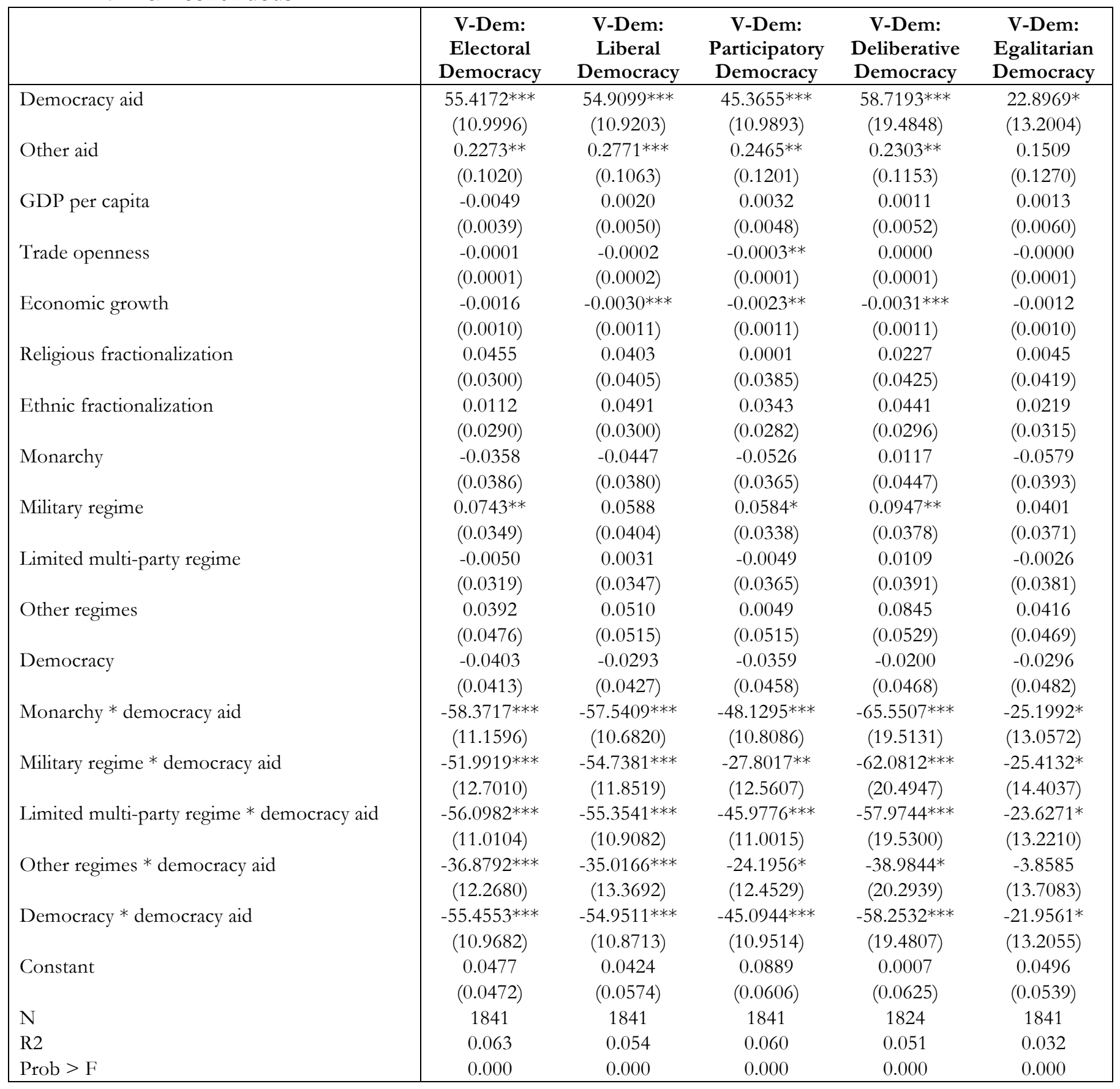


V-Dem ordinal

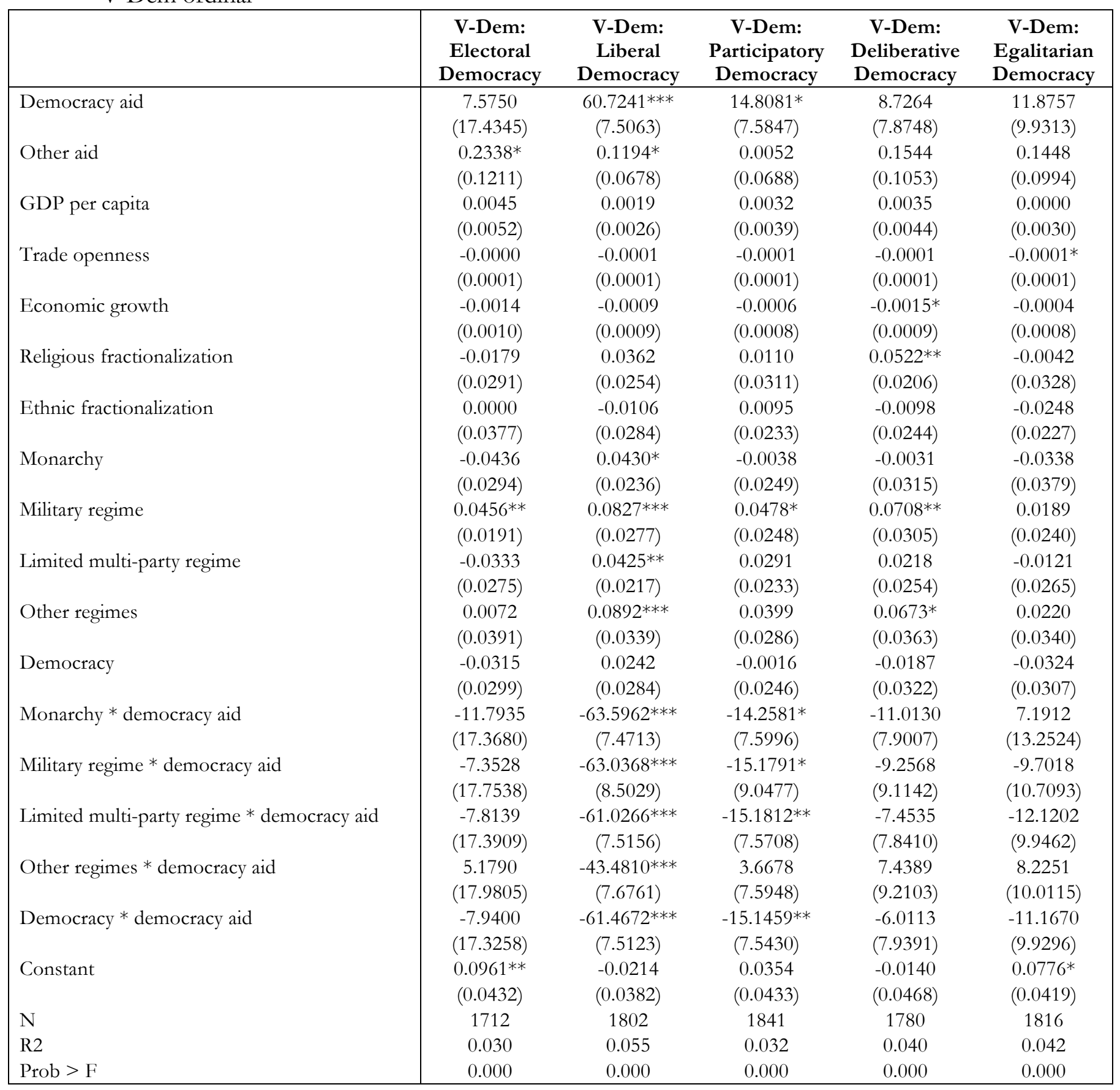


b) AUT only

\begin{tabular}{|c|c|c|c|c|}
\hline & $\begin{array}{l}\text { Freedom } \\
\text { House } \\
\text { (FIW, any } \\
\text { change) }\end{array}$ & $\begin{array}{c}\text { Freedom } \\
\text { House (PR, } \\
\text { any change) }\end{array}$ & $\begin{array}{l}\text { Freedom } \\
\text { House } \\
\text { (FIW: } \\
\text { 1+points) }\end{array}$ & $\begin{array}{c}\text { Freedom } \\
\text { House (PR: } \\
\text { 1+points) }\end{array}$ \\
\hline Democracy aid & $\begin{array}{c}62.2380 * * * \\
(15.4711)\end{array}$ & $\begin{array}{c}19.2872 \\
(21.8863)\end{array}$ & $\begin{array}{c}57.9110 * * * \\
(12.7137)\end{array}$ & $\begin{array}{c}19.2872 \\
(21.8863)\end{array}$ \\
\hline Other aid & $\begin{array}{c}0.2215 \\
(0.3195)\end{array}$ & $\begin{array}{c}0.1420 \\
(0.2085)\end{array}$ & $\begin{array}{c}0.0259 \\
(0.2110)\end{array}$ & $\begin{array}{c}0.1420 \\
(0.2085)\end{array}$ \\
\hline GDP per capita & $\begin{array}{l}-0.0030 \\
(0.0106)\end{array}$ & $\begin{array}{c}0.0012 \\
(0.0081)\end{array}$ & $\begin{array}{c}0.0025 \\
(0.0073)\end{array}$ & $\begin{array}{c}0.0012 \\
(0.0081)\end{array}$ \\
\hline Trade openness & $\begin{array}{c}0.0001 \\
(0.0002)\end{array}$ & $\begin{array}{c}0.0001 \\
(0.0002)\end{array}$ & $\begin{array}{c}0.0002 \\
(0.0002)\end{array}$ & $\begin{array}{c}0.0001 \\
(0.0002)\end{array}$ \\
\hline Economic growth & $\begin{array}{c}-0.0046^{* * *} \\
(0.0012)\end{array}$ & $\begin{array}{c}-0.0030^{* *} \\
(0.0012)\end{array}$ & $\begin{array}{c}-0.0022 * * \\
(0.0009)\end{array}$ & $\begin{array}{c}-0.0030^{* *} \\
(0.0012)\end{array}$ \\
\hline Religious fractionalization & $\begin{array}{c}0.0462 \\
(0.0581)\end{array}$ & $\begin{array}{c}0.0131 \\
(0.0530)\end{array}$ & $\begin{array}{c}0.0036 \\
(0.0334)\end{array}$ & $\begin{array}{c}0.0131 \\
(0.0530)\end{array}$ \\
\hline Ethnic fractionalization & $\begin{array}{c}0.1117 * * * \\
(0.0392)\end{array}$ & $\begin{array}{l}0.0643^{*} \\
(0.0368)\end{array}$ & $\begin{array}{c}0.0626^{* *} \\
(0.0294)\end{array}$ & $\begin{array}{l}0.0643^{*} \\
(0.0368)\end{array}$ \\
\hline Monarchy & $\begin{array}{c}0.0739 \\
(0.0815)\end{array}$ & $\begin{array}{c}0.0633 \\
(0.0570)\end{array}$ & $\begin{array}{l}-0.0159 \\
(0.0470)\end{array}$ & $\begin{array}{c}0.0633 \\
(0.0570)\end{array}$ \\
\hline Military regime & $\begin{array}{c}0.1102^{* *} \\
(0.0561)\end{array}$ & $\begin{array}{c}0.1018^{* *} \\
(0.0402)\end{array}$ & $\begin{array}{c}0.0431 \\
(0.0391)\end{array}$ & $\begin{array}{c}0.1018^{* *} \\
(0.0402)\end{array}$ \\
\hline Limited multi-party regime & $\begin{array}{c}0.0582 \\
(0.0576)\end{array}$ & $\begin{array}{c}0.0702 \\
(0.0462)\end{array}$ & $\begin{array}{c}0.0007 \\
(0.0370)\end{array}$ & $\begin{array}{c}0.0702 \\
(0.0462)\end{array}$ \\
\hline Other regimes & $\begin{array}{c}0.1514 * * \\
(0.0695)\end{array}$ & $\begin{array}{c}0.1680^{* * *} \\
(0.0528)\end{array}$ & $\begin{array}{c}0.0818 \\
(0.0499)\end{array}$ & $\begin{array}{c}0.1680^{* * *} \\
(0.0528)\end{array}$ \\
\hline Democracy & $\begin{array}{c}0.0000 \\
(.)\end{array}$ & $\begin{array}{l}-0.2389 \\
(0.7665)\end{array}$ & $\begin{array}{c}0.0000 \\
(.)\end{array}$ & $\begin{array}{l}-0.2389 \\
(0.7665)\end{array}$ \\
\hline Monarchy $*$ democracy aid & $\begin{array}{c}-64.8148^{* * *} \\
(16.4438)\end{array}$ & $\begin{array}{l}-25.4021 \\
(21.0521)\end{array}$ & $\begin{array}{c}-63.4593 * * * \\
(14.0561)\end{array}$ & $\begin{array}{l}-25.4021 \\
(21.0521)\end{array}$ \\
\hline Military regime $*$ democracy aid & $\begin{array}{c}-63.1326 * * * \\
(19.3258)\end{array}$ & $\begin{array}{l}-17.3253 \\
(23.6924)\end{array}$ & $\begin{array}{c}-62.6574 * * * \\
(12.9608)\end{array}$ & $\begin{array}{l}-17.3253 \\
(23.6924)\end{array}$ \\
\hline Limited multi-party regime ${ }^{*}$ democracy aid & $\begin{array}{c}-55.8983^{* * *} \\
(15.3659)\end{array}$ & $\begin{array}{l}-19.3321 \\
(21.8020)\end{array}$ & $\begin{array}{c}-56.7759 * * * \\
(12.9078)\end{array}$ & $\begin{array}{l}-19.3321 \\
(21.8020)\end{array}$ \\
\hline Other regimes $*$ democracy aid & $\begin{array}{c}-47.0343^{* * *} \\
(15.9694)\end{array}$ & $\begin{array}{c}-2.6987 \\
(21.3729)\end{array}$ & $\begin{array}{c}-59.4919 * * * \\
(13.6634)\end{array}$ & $\begin{array}{c}-2.6987 \\
(21.3729)\end{array}$ \\
\hline Democracy $*$ democracy aid & $\begin{array}{c}0.0000 \\
(.)\end{array}$ & $\begin{array}{l}-14.5390 \\
(100.0123)\end{array}$ & $\begin{array}{c}0.0000 \\
(.)\end{array}$ & $\begin{array}{l}-14.5390 \\
(100.0123)\end{array}$ \\
\hline Constant & $\begin{array}{c}0.1358 \\
(0.1240)\end{array}$ & $\begin{array}{c}0.1335 \\
(0.1067)\end{array}$ & $\begin{array}{c}0.2193^{* *} \\
(0.1091)\end{array}$ & $\begin{array}{c}0.1335 \\
(0.1067)\end{array}$ \\
\hline $\mathrm{N}$ & 1090 & 1137 & 1090 & 1137 \\
\hline $\mathrm{R} 2$ & 0.061 & 0.054 & 0.061 & 0.054 \\
\hline Prob $>$ F & 0.000 & 0.000 & 0.000 & 0.000 \\
\hline
\end{tabular}




\begin{tabular}{|c|c|c|c|c|c|}
\hline & $\begin{array}{c}\text { Gasiorowski } \\
\text { (hybrid) }\end{array}$ & $\begin{array}{c}\text { Gates et al. } \\
2006 \text { (any } \\
\text { change) }\end{array}$ & $\begin{array}{l}\text { Polity IV } \\
\text { (any } \\
\text { change) }\end{array}$ & $\begin{array}{c}\text { Polity IV } \\
(3+\text { points })\end{array}$ & $\begin{array}{c}\text { Polity IV } \\
\text { (5+ points) }\end{array}$ \\
\hline Democracy aid & $\begin{array}{c}\text { no } \\
\text { observations }\end{array}$ & $\begin{array}{c}32.9746 \\
(34.1309)\end{array}$ & $\begin{array}{c}41.4601 \\
(32.0147)\end{array}$ & $\begin{array}{c}32.9637 \\
(24.8707)\end{array}$ & $\begin{array}{l}40.8277^{*} \\
(22.2834)\end{array}$ \\
\hline Other aid & & $\begin{array}{c}0.0895 \\
(0.3832)\end{array}$ & $\begin{array}{c}0.1342 \\
(0.3686)\end{array}$ & $\begin{array}{c}0.1579 \\
(0.2774)\end{array}$ & $\begin{array}{c}0.1500 \\
(0.2944)\end{array}$ \\
\hline Trade openness & & $\begin{array}{l}-0.0001 \\
(0.0002)\end{array}$ & $\begin{array}{c}-0.0003 * \\
(0.0002)\end{array}$ & $\begin{array}{l}-0.0002 \\
(0.0001)\end{array}$ & $\begin{array}{l}-0.0000 \\
(0.0001)\end{array}$ \\
\hline Economic growth & & $\begin{array}{c}-0.0024^{* *} \\
(0.0012)\end{array}$ & $\begin{array}{l}-0.0018 \\
(0.0012)\end{array}$ & $\begin{array}{l}-0.0013 \\
(0.0009)\end{array}$ & $\begin{array}{l}-0.0007 \\
(0.0009)\end{array}$ \\
\hline Religious fractionalization & & $\begin{array}{c}0.0285 \\
(0.0533)\end{array}$ & $\begin{array}{l}-0.0372 \\
(0.0595)\end{array}$ & $\begin{array}{l}-0.0135 \\
(0.0477)\end{array}$ & $\begin{array}{l}-0.0183 \\
(0.0414)\end{array}$ \\
\hline Monarchy & & $\begin{array}{l}-0.0496 \\
(0.0596)\end{array}$ & $\begin{array}{l}-0.0671 \\
(0.0808)\end{array}$ & $\begin{array}{l}-0.0093 \\
(0.0431)\end{array}$ & $\begin{array}{c}0.0213 \\
(0.0463)\end{array}$ \\
\hline Military regime & & $\begin{array}{c}0.0450 \\
(0.0539)\end{array}$ & $\begin{array}{c}0.0162 \\
(0.0611)\end{array}$ & $\begin{array}{c}0.0707 \\
(0.0487)\end{array}$ & $\begin{array}{c}0.0649 \\
(0.0437)\end{array}$ \\
\hline Limited multi-party regime & & $\begin{array}{c}0.0360 \\
(0.0450)\end{array}$ & $\begin{array}{l}-0.0696 \\
(0.0688)\end{array}$ & $\begin{array}{c}0.0273 \\
(0.0387)\end{array}$ & $\begin{array}{c}0.0320 \\
(0.0400)\end{array}$ \\
\hline Other regimes & & $\begin{array}{l}0.1752^{*} \\
(0.1063)\end{array}$ & $\begin{array}{l}-0.0819 \\
(0.0908)\end{array}$ & $\begin{array}{l}-0.0352 \\
(0.0628)\end{array}$ & $\begin{array}{c}0.0073 \\
(0.0575)\end{array}$ \\
\hline Democracy & & $\begin{array}{c}0.0000 \\
(.)\end{array}$ & $\begin{array}{c}0.0000 \\
(.)\end{array}$ & $\begin{array}{c}0.0000 \\
(.)\end{array}$ & $\begin{array}{c}0.0000 \\
(.)\end{array}$ \\
\hline Monarchy * democracy aid & & $\begin{array}{l}-39.0597 \\
(33.5176)\end{array}$ & $\begin{array}{l}-45.4738 \\
(33.0960)\end{array}$ & $\begin{array}{l}-36.1656 \\
(25.6349)\end{array}$ & $\begin{array}{c}-44.4556^{*} \\
(23.3946)\end{array}$ \\
\hline Democracy $*$ democracy aid & & $\begin{array}{c}0.0000 \\
(.)\end{array}$ & $\begin{array}{c}0.0000 \\
(.)\end{array}$ & $\begin{array}{c}0.0000 \\
(.)\end{array}$ & $\begin{array}{c}0.0000 \\
(.)\end{array}$ \\
\hline Constant & & $\begin{array}{c}0.1974 \\
(0.1414)\end{array}$ & $\begin{array}{c}0.5512^{* * *} \\
(0.1623)\end{array}$ & $\begin{array}{c}0.3869 * * \\
(0.1636)\end{array}$ & $\begin{array}{l}0.2001^{*} \\
(0.1158)\end{array}$ \\
\hline $\mathrm{N}$ & & 470 & 799 & 799 & 799 \\
\hline $\mathrm{R} 2$ & & 0.059 & 0.072 & 0.056 & 0.043 \\
\hline Prob $>$ F & & 0.000 & 0.000 & 0.000 & 0.000 \\
\hline
\end{tabular}




\begin{tabular}{|c|c|c|c|c|}
\hline & $\begin{array}{c}\text { Reich } 2002 \\
\text { (hybrid) }\end{array}$ & $\begin{array}{l}\text { Skaaning et } \\
\text { al. } 2015\end{array}$ & $\begin{array}{l}\text { Wahman et } \\
\text { al. } 2013 \text { (any } \\
\text { change) }\end{array}$ & $\begin{array}{l}\text { Wahman et } \\
\text { al. } 2013 \\
\text { (1.5+ points) }\end{array}$ \\
\hline Democracy aid & $\begin{array}{c}\text { no } \\
\text { observations }\end{array}$ & $\begin{array}{l}\text { no thresholds } \\
\text { for DEM/ }\end{array}$ & $\begin{array}{c}67.5590 * * * \\
(16.3281)\end{array}$ & $\begin{array}{c}53.7257 * * * \\
(9.0427)\end{array}$ \\
\hline Other aid & & AUT & $\begin{array}{c}0.0651 \\
(0.1499)\end{array}$ & $\begin{array}{c}0.0350 \\
(0.0869)\end{array}$ \\
\hline GDP per capita & & & $\begin{array}{c}0.0087 \\
(0.0071)\end{array}$ & $\begin{array}{c}0.0012 \\
(0.0024)\end{array}$ \\
\hline Trade openness & & & $\begin{array}{l}-0.0000 \\
(0.0002)\end{array}$ & $\begin{array}{l}-0.0000 \\
(0.0001)\end{array}$ \\
\hline Economic growth & & & $\begin{array}{c}-0.0054 * * * \\
(0.0013)\end{array}$ & $\begin{array}{c}-0.0015^{* *} \\
(0.0007)\end{array}$ \\
\hline Religious fractionalization & & & $\begin{array}{c}0.0255 \\
(0.0534)\end{array}$ & $\begin{array}{c}0.0024 \\
(0.0266)\end{array}$ \\
\hline Ethnic fractionalization & & & $\begin{array}{c}0.0443 \\
(0.0308)\end{array}$ & $\begin{array}{c}0.0019 \\
(0.0182)\end{array}$ \\
\hline Monarchy & & & $\begin{array}{c}0.0436 \\
(0.0698)\end{array}$ & $\begin{array}{c}0.0054 \\
(0.0239)\end{array}$ \\
\hline Military regime & & & $\begin{array}{c}0.1567 * * * \\
(0.0474)\end{array}$ & $\begin{array}{c}0.0606^{* *} \\
(0.0273)\end{array}$ \\
\hline Limited multi-party regime & & & $\begin{array}{c}0.0580 \\
(0.0473)\end{array}$ & $\begin{array}{c}0.0075 \\
(0.0222)\end{array}$ \\
\hline Other regimes & & & $\begin{array}{c}0.1295^{* *} \\
(0.0590)\end{array}$ & $\begin{array}{c}0.0656^{* *} \\
(0.0333)\end{array}$ \\
\hline Democracy & & & $\begin{array}{l}-0.0529 \\
(0.0605)\end{array}$ & $\begin{array}{l}-0.0289 \\
(0.0266)\end{array}$ \\
\hline Monarchy $*$ democracy aid & & & $\begin{array}{c}-72.1734^{* * * *} \\
(17.5600)\end{array}$ & $\begin{array}{c}-55.3920^{* * * *} \\
(9.0603)\end{array}$ \\
\hline Military regime $*$ democracy aid & & & $\begin{array}{c}-58.1747 * * * \\
(19.2091)\end{array}$ & $\begin{array}{c}-56.8549 * * * \\
(10.4651)\end{array}$ \\
\hline Limited multi-party regime $*$ democracy aid & & & $\begin{array}{c}-65.9406^{* * *} \\
(16.4120)\end{array}$ & $\begin{array}{c}-54.0792^{* * *} \\
(9.0517)\end{array}$ \\
\hline Other regimes $*$ democracy aid & & & $\begin{array}{c}-49.3876^{* * * *} \\
(17.1713)\end{array}$ & $\begin{array}{c}-53.1112^{* * *} \\
(9.8640)\end{array}$ \\
\hline Democracy $*$ democracy aid & & & $\begin{array}{c}-68.5522^{* * *} \\
(16.3272)\end{array}$ & $\begin{array}{c}-53.4936 * * * \\
(9.0448)\end{array}$ \\
\hline Constant & & & $\begin{array}{c}0.1053 \\
(0.0847)\end{array}$ & $\begin{array}{c}0.0900 * * \\
(0.0457)\end{array}$ \\
\hline $\mathrm{N}$ & & & 1921 & 1766 \\
\hline $\mathrm{R} 2$ & & & 0.072 & 0.058 \\
\hline Prob $>$ F & & & 0.000 & 0.000 \\
\hline
\end{tabular}


Appendix X: Robustness Checks: Ansell and Samuels 2010

a) all potential reformers

\begin{tabular}{|c|c|c|c|c|c|c|c|c|}
\hline & \multicolumn{2}{|c|}{$\begin{array}{c}\text { Freedom House (FIW, any } \\
\text { change) }\end{array}$} & \multicolumn{2}{|c|}{$\begin{array}{c}\text { Freedom House (PR, any } \\
\text { change) }\end{array}$} & \multicolumn{2}{|c|}{$\begin{array}{c}\text { Freedom House (FIW, 1+ } \\
\text { points) }\end{array}$} & \multicolumn{2}{|c|}{$\begin{array}{c}\text { Freedom House (PR, 1+ } \\
\text { points) }\end{array}$} \\
\hline & T3, MC & T3, ME & T3, MC & T3, ME & T3, MC & T3, ME & T3, MC & T3, ME \\
\hline Lag Democracy Score & $\begin{array}{c}0.0854 * * * \\
(0.0111)\end{array}$ & $\begin{array}{c}0.0898 * * * \\
(0.0093)\end{array}$ & $\begin{array}{c}0.0622 * * * \\
(0.0079)\end{array}$ & $\begin{array}{c}0.0642 * * * \\
(0.0073)\end{array}$ & $\begin{array}{c}0.0299 * * * \\
(0.0077)\end{array}$ & $\begin{array}{l}0.0246^{* *} \\
(0.0098)\end{array}$ & $\begin{array}{c}0.0416^{* * *} \\
(0.0142)\end{array}$ & $\begin{array}{c}0.1021 \\
(0.0801)\end{array}$ \\
\hline Gini & $\begin{array}{l}-0.2351 \\
(0.5252)\end{array}$ & $\begin{array}{c}6.6289 * * \\
(3.2298)\end{array}$ & $\begin{array}{l}-0.2820 \\
(0.4178)\end{array}$ & $\begin{array}{l}7.5098^{*} \\
(3.9553)\end{array}$ & $\begin{array}{c}0.4285 \\
(0.4768)\end{array}$ & $\begin{array}{l}10.5823 \\
(9.7770)\end{array}$ & $\begin{array}{l}-0.0679 \\
(0.6214)\end{array}$ & $\begin{array}{l}-86.9676 \\
(96.8753)\end{array}$ \\
\hline GDP per capita & $\begin{array}{c}-0.0000^{* *} \\
(0.0000)\end{array}$ & $\begin{array}{c}-0.0000^{*} \\
(0.0000)\end{array}$ & $\begin{array}{l}-0.0000 \\
(0.0000)\end{array}$ & $\begin{array}{c}0.0000 \\
(0.0000)\end{array}$ & $\begin{array}{l}-0.0000 \\
(0.0000)\end{array}$ & $\begin{array}{l}-0.0000 \\
(0.0000)\end{array}$ & $\begin{array}{l}-0.0000 \\
(0.0000)\end{array}$ & $\begin{array}{c}0.0001 \\
(0.0001)\end{array}$ \\
\hline Rural inequality & $\begin{array}{l}-0.1046 \\
(0.1540)\end{array}$ & $\begin{array}{l}-0.0868 \\
(0.1431)\end{array}$ & $\begin{array}{l}-0.0571 \\
(0.1319)\end{array}$ & $\begin{array}{l}-0.0327 \\
(0.1324)\end{array}$ & $\begin{array}{l}-0.0923 \\
(0.1086)\end{array}$ & $\begin{array}{l}-0.0502 \\
(0.1215)\end{array}$ & $\begin{array}{l}-0.0668 \\
(0.1351)\end{array}$ & $\begin{array}{l}-0.4442 \\
(0.7051)\end{array}$ \\
\hline Vanhanen Knowledge & $\begin{array}{c}-0.0075^{* *} \\
(0.0031)\end{array}$ & $\begin{array}{c}-0.0065^{* * *} \\
(0.0022)\end{array}$ & $\begin{array}{c}-0.0102^{* * *} \\
(0.0025)\end{array}$ & $\begin{array}{c}-0.0092^{* * *} \\
(0.0021)\end{array}$ & $\begin{array}{c}-0.0054 * * \\
(0.0022)\end{array}$ & $\begin{array}{c}-0.0053^{* * * *} \\
(0.0019)\end{array}$ & $\begin{array}{c}-0.0109 * * * \\
(0.0035)\end{array}$ & $\begin{array}{l}-0.0078 \\
(0.0105)\end{array}$ \\
\hline Year & $\begin{array}{c}0.0090^{* * *} \\
(0.0027)\end{array}$ & $\begin{array}{c}0.0066^{* *} \\
(0.0026)\end{array}$ & $\begin{array}{c}0.0109 * * * \\
(0.0024)\end{array}$ & $\begin{array}{c}0.0075^{* * *} \\
(0.0028)\end{array}$ & $\begin{array}{l}0.0046^{* *} \\
(0.0019)\end{array}$ & $\begin{array}{c}0.0012 \\
(0.0038)\end{array}$ & $\begin{array}{c}0.0121 * * * \\
(0.0027)\end{array}$ & $\begin{array}{c}0.0244 \\
(0.0172)\end{array}$ \\
\hline Constant & $\begin{array}{c}-17.5376^{* * *} \\
(5.1626)\end{array}$ & $\begin{array}{c}-15.8858^{* * *} \\
(4.7781)\end{array}$ & $\begin{array}{c}-21.2548^{* * *} \\
(4.7606)\end{array}$ & $\begin{array}{c}-18.1433^{* * *} \\
(4.7370)\end{array}$ & $\begin{array}{c}-9.1126^{* *} \\
(3.7953)\end{array}$ & $\begin{array}{l}-7.1304 \\
(4.4212)\end{array}$ & $\begin{array}{c}-23.7043^{* * *} \\
(5.3742)\end{array}$ & $\begin{array}{c}-8.8248 \\
(26.3605)\end{array}$ \\
\hline $\mathrm{N}$ & 2207 & 2206 & 1999 & 1998 & 1834 & 1833 & 1587 & 1587 \\
\hline $\mathrm{R} 2$ & 0.050 & & 0.056 & & 0.020 & & 0.035 & \\
\hline Prob $>$ F & 0.000 & 0.000 & 0.000 & 0.000 & 0.000 & 0.282 & 0.000 & 1.000 \\
\hline
\end{tabular}




\begin{tabular}{|c|c|c|c|c|c|c|c|c|c|c|}
\hline & \multicolumn{2}{|c|}{ Gasiorowski 1996 (hybrid) } & \multicolumn{2}{|c|}{$\begin{array}{c}\text { Gates et al. } 2006 \text { (any } \\
\text { change) }\end{array}$} & \multicolumn{2}{|c|}{ Polity IV (any change) } & \multicolumn{2}{|c|}{ Polity IV (3+ points) } & \multicolumn{2}{|c|}{ Polity IV ( $5+$ points) } \\
\hline & T3, MC & T3, ME & T3, MC & T3, ME & T3, MC & T3, ME & T3, MC & T3, ME & T3, MC & T3, ME \\
\hline Lag Democracy Score & $\begin{array}{c}-0.0224 * * \\
(0.0085)\end{array}$ & $\begin{array}{c}-0.0243 * * * \\
(0.0063)\end{array}$ & $\begin{array}{c}-0.1610^{* * * *} \\
(0.0211)\end{array}$ & $\begin{array}{c}-0.1606^{* * *} \\
(0.0162)\end{array}$ & $\begin{array}{c}-0.0099 * * * \\
(0.0017)\end{array}$ & $\begin{array}{c}-0.0103^{* * *} \\
(0.0014)\end{array}$ & $\begin{array}{c}-0.0058^{* * *} \\
(0.0019)\end{array}$ & $\begin{array}{c}-0.0057 * * * \\
(0.0014)\end{array}$ & $\begin{array}{l}-0.0025 \\
(0.0022)\end{array}$ & $\begin{array}{l}-0.0025^{*} \\
(0.0015)\end{array}$ \\
\hline Gini & $\begin{array}{c}0.2866 \\
(0.2122)\end{array}$ & $\begin{array}{l}-1.4650 \\
(1.6875)\end{array}$ & $\begin{array}{c}0.1197 \\
(0.1711)\end{array}$ & $\begin{array}{l}1.1520^{*} \\
(0.6888)\end{array}$ & $\begin{array}{c}0.0446 \\
(0.1545)\end{array}$ & $\begin{array}{c}0.8302 \\
(0.8912)\end{array}$ & $\begin{array}{c}0.1723 \\
(0.1945)\end{array}$ & $\begin{array}{c}1.0149 \\
(0.9478)\end{array}$ & $\begin{array}{l}0.1799 \\
(0.1802)\end{array}$ & $\begin{array}{l}0.4370 \\
(0.8511)\end{array}$ \\
\hline GDP per capita & $\begin{array}{l}-0.0000 \\
(0.0000)\end{array}$ & $\begin{array}{l}0.0000 \\
(0.0000)\end{array}$ & $\begin{array}{c}-0.0000^{* * *} \\
(0.0000)\end{array}$ & $\begin{array}{c}-0.0000^{* *} \\
(0.0000)\end{array}$ & $\begin{array}{l}0.0000 \\
(0.0000)\end{array}$ & $\begin{array}{c}0.0000 \\
(0.0000)\end{array}$ & $\begin{array}{c}0.0000 \\
(0.0000)\end{array}$ & $\begin{array}{c}0.0000 \\
(0.0000)\end{array}$ & $\begin{array}{c}0.0000^{* *} \\
(0.0000)\end{array}$ & $\begin{array}{c}0.0000 * * * \\
(0.0000)\end{array}$ \\
\hline Rural inequality & $\begin{array}{l}0.0167 \\
(0.0289)\end{array}$ & $\begin{array}{l}-0.0277 \\
(0.0574)\end{array}$ & $\begin{array}{c}-0.0960^{* *} \\
(0.0477)\end{array}$ & $\begin{array}{c}-0.0888^{*} \\
(0.0469)\end{array}$ & $\begin{array}{l}-0.1010^{*} \\
(0.0510)\end{array}$ & $\begin{array}{l}-0.0743 \\
(0.0737)\end{array}$ & $\begin{array}{l}-0.0661 \\
(0.0518)\end{array}$ & $\begin{array}{l}-0.0320 \\
(0.0693)\end{array}$ & $\begin{array}{l}-0.0696 \\
(0.0485)\end{array}$ & $\begin{array}{l}-0.0587 \\
(0.0624)\end{array}$ \\
\hline Vanhanen Knowledge & $\begin{array}{c}0.0001 \\
(0.0009)\end{array}$ & $\begin{array}{l}-0.0003 \\
(0.0008)\end{array}$ & $\begin{array}{c}-0.0027 * * * \\
(0.0008)\end{array}$ & $\begin{array}{c}-0.0024^{* * *} \\
(0.0008)\end{array}$ & $\begin{array}{c}-0.0031 * * \\
(0.0015)\end{array}$ & $\begin{array}{c}-0.0030^{* *} \\
(0.0012)\end{array}$ & $\begin{array}{c}-0.0035^{* *} \\
(0.0014)\end{array}$ & $\begin{array}{c}-0.0031 * * \\
(0.0012)\end{array}$ & $\begin{array}{c}-0.0043 * * * \\
(0.0012)\end{array}$ & $\begin{array}{c}-0.0042^{* * *} \\
(0.0011)\end{array}$ \\
\hline Year & $\begin{array}{l}0.0008 \\
(0.0006)\end{array}$ & $\begin{array}{l}0.0000 \\
(0.0010)\end{array}$ & $\begin{array}{c}0.0032^{* * *} \\
(0.0009)\end{array}$ & $\begin{array}{c}0.0031 * * * \\
(0.0007)\end{array}$ & $\begin{array}{c}0.0046^{* * *} \\
(0.0014)\end{array}$ & $\begin{array}{c}0.0047 * * * \\
(0.0011)\end{array}$ & $\begin{array}{c}0.0040 * * * \\
(0.0014)\end{array}$ & $\begin{array}{c}0.0042 * * * \\
(0.0010)\end{array}$ & $\begin{array}{c}0.0040 * * * \\
(0.0012)\end{array}$ & $\begin{array}{c}0.0041 * * * \\
(0.0009)\end{array}$ \\
\hline Constant & $\begin{array}{l}-1.6174 \\
(1.2463)\end{array}$ & $\begin{array}{l}0.6881 \\
(2.5596)\end{array}$ & $\begin{array}{c}-6.1236^{* * * *} \\
(1.6909)\end{array}$ & $\begin{array}{c}-6.2959 * * * \\
(1.3949)\end{array}$ & $\begin{array}{c}-8.8343^{* * * *} \\
(2.7910)\end{array}$ & $\begin{array}{c}-9.5434 * * * \\
(2.2870)\end{array}$ & $\begin{array}{c}-7.8162^{* * * *} \\
(2.7879)\end{array}$ & $\begin{array}{c}-8.6820^{* * *} \\
(2.1923)\end{array}$ & $\begin{array}{c}-7.9346 * * * \\
(2.2896)\end{array}$ & $\begin{array}{c}-8.1177 * * * \\
(1.9261)\end{array}$ \\
\hline $\mathrm{N}$ & 1949 & 1949 & 3602 & 3601 & 2948 & 2947 & 2512 & 2511 & 2265 & 2265 \\
\hline $\mathrm{R} 2$ & 0.014 & & 0.033 & & 0.029 & & 0.022 & & 0.033 & \\
\hline Prob $>$ F & 0.135 & 0.999 & 0.000 & 0.000 & 0.000 & 0.000 & 0.000 & 0.000 & 0.000 & 0.000 \\
\hline
\end{tabular}




\begin{tabular}{|c|c|c|c|c|c|c|c|c|}
\hline & \multicolumn{2}{|c|}{ Reich 2002 (hybrid) } & \multicolumn{2}{|c|}{ Skaaning et al. 2015} & \multicolumn{2}{|c|}{$\begin{array}{c}\text { Wahman et al. } 2013 \text { (any } \\
\text { change) }\end{array}$} & \multicolumn{2}{|c|}{$\begin{array}{c}\text { Wahman et al. } 2013(1.5+ \\
\text { points) }\end{array}$} \\
\hline & T3, MC & T3, ME & T3, MC & T3, ME & T3, MC & T3, ME & T3, MC & T3, ME \\
\hline Lag Democracy Score & $\begin{array}{c}-0.0201 * * * \\
(0.0056)\end{array}$ & $\begin{array}{c}-0.0197 * * * \\
(0.0038)\end{array}$ & $\begin{array}{c}-0.0474 * * \\
(0.0200)\end{array}$ & $\begin{array}{c}0.1847 \\
(0.5082)\end{array}$ & $\begin{array}{c}-0.0481 * * * \\
(0.0061)\end{array}$ & $\begin{array}{c}-0.0521 * * * \\
(0.0060)\end{array}$ & $\begin{array}{c}-0.0234 * * * \\
(0.0044)\end{array}$ & $\begin{array}{c}-0.0242 * * * \\
(0.0032)\end{array}$ \\
\hline Gini & $\begin{array}{c}0.0670 \\
(0.0825)\end{array}$ & $\begin{array}{l}-0.1183 \\
(0.2591)\end{array}$ & $\begin{array}{l}-0.2230 \\
(0.9169)\end{array}$ & $\begin{array}{c}114.2926 \\
(248.6343)\end{array}$ & $\begin{array}{l}-0.3103 \\
(0.4936)\end{array}$ & $\begin{array}{l}7.0360 * * \\
(3.5409)\end{array}$ & $\begin{array}{c}0.4131 \\
(0.3062)\end{array}$ & $\begin{array}{c}4.7208 \\
(2.8768)\end{array}$ \\
\hline GDP per capita & $\begin{array}{c}-0.0000 * * \\
(0.0000)\end{array}$ & $\begin{array}{c}-0.0000^{* *} \\
(0.0000)\end{array}$ & $\begin{array}{l}-0.0000 \\
(0.0000)\end{array}$ & $\begin{array}{l}-0.0001 \\
(0.0003)\end{array}$ & $\begin{array}{c}-0.0000^{* * *} \\
(0.0000)\end{array}$ & $\begin{array}{c}-0.0000 * * \\
(0.0000)\end{array}$ & $\begin{array}{l}-0.0000 \\
(0.0000)\end{array}$ & $\begin{array}{l}-0.0000 \\
(0.0000)\end{array}$ \\
\hline Rural inequality & $\begin{array}{l}-0.0059 \\
(0.0332)\end{array}$ & $\begin{array}{l}-0.0123 \\
(0.0273)\end{array}$ & $\begin{array}{l}-0.0869 \\
(0.1388)\end{array}$ & $\begin{array}{c}0.6900 \\
(1.8286)\end{array}$ & $\begin{array}{l}-0.1556 \\
(0.1677)\end{array}$ & $\begin{array}{l}-0.1229 \\
(0.1522)\end{array}$ & $\begin{array}{l}-0.0408 \\
(0.1051)\end{array}$ & $\begin{array}{l}-0.0319 \\
(0.0839)\end{array}$ \\
\hline Vanhanen Knowledge & $\begin{array}{l}-0.0004 \\
(0.0004)\end{array}$ & $\begin{array}{l}-0.0005 \\
(0.0004)\end{array}$ & $\begin{array}{c}-0.0120 * * \\
(0.0049)\end{array}$ & $\begin{array}{l}-0.0066 \\
(0.0179)\end{array}$ & $\begin{array}{c}-0.0082 * * * \\
(0.0030)\end{array}$ & $\begin{array}{c}-0.0073 * * * \\
(0.0023)\end{array}$ & $\begin{array}{c}-0.0052 * * * \\
(0.0017)\end{array}$ & $\begin{array}{c}-0.0048^{* * *} \\
(0.0013)\end{array}$ \\
\hline Year & $\begin{array}{l}0.0012^{* *} \\
(0.0005)\end{array}$ & $\begin{array}{l}0.0012 * * * \\
(0.0004)\end{array}$ & $\begin{array}{c}0.0187 * * * \\
(0.0038)\end{array}$ & $\begin{array}{l}-0.0024 \\
(0.0480)\end{array}$ & $\begin{array}{c}0.0121 * * * \\
(0.0030)\end{array}$ & $\begin{array}{c}0.0101 * * * \\
(0.0028)\end{array}$ & $\begin{array}{c}0.0059 * * * \\
(0.0017)\end{array}$ & $\begin{array}{l}0.0041^{* *} \\
(0.0019)\end{array}$ \\
\hline Constant & $\begin{array}{c}-2.2397 * * \\
(1.0181)\end{array}$ & $\begin{array}{c}-2.1474 * * * \\
(0.7971)\end{array}$ & $\begin{array}{c}-36.2772^{* * *} \\
(7.4536)\end{array}$ & $\begin{array}{l}-47.1822 \\
(36.3747)\end{array}$ & $\begin{array}{c}-22.9262^{* * *} \\
(5.7762)\end{array}$ & $\begin{array}{c}-22.3818^{* * *} \\
(5.1779)\end{array}$ & $\begin{array}{c}-11.5615^{* * * *} \\
(3.3009)\end{array}$ & $\begin{array}{c}-9.9160 \text { *** } \\
(3.1340)\end{array}$ \\
\hline $\mathrm{N}$ & 3265 & 3265 & 1386 & 1386 & 2215 & 2214 & 1968 & 1967 \\
\hline $\mathrm{R} 2$ & 0.011 & & 0.048 & & 0.044 & & 0.039 & \\
\hline Prob $>$ F & 0.013 & 0.971 & 0.000 & 1.000 & 0.000 & 0.000 & 0.000 & 0.000 \\
\hline
\end{tabular}




\begin{tabular}{|c|c|c|c|c|c|c|c|c|c|c|}
\hline & \multicolumn{2}{|c|}{$\begin{array}{l}\text { V-Dem: Electoral } \\
\text { Democracy }\end{array}$} & \multicolumn{2}{|c|}{ V-Dem: Liberal Democracy } & \multicolumn{2}{|c|}{$\begin{array}{c}\text { V-Dem: Participatory } \\
\text { Democracy }\end{array}$} & \multicolumn{2}{|c|}{$\begin{array}{c}\text { V-Dem: Deliberative } \\
\text { Democracy }\end{array}$} & \multicolumn{2}{|c|}{$\begin{array}{c}\text { V-Dem: Egalitarian } \\
\text { Democracy }\end{array}$} \\
\hline & T3, MC & T3, ME & T3, MC & T3, ME & T3, MC & T3, ME & T3, MC & T3, ME & T3, MC & T3, ME \\
\hline Lag Democracy Score & $\begin{array}{c}-0.2357 * * * \\
(0.0421)\end{array}$ & $\begin{array}{c}-0.2369 * * * \\
(0.0298)\end{array}$ & $\begin{array}{c}-0.1500 * * * \\
(0.0404)\end{array}$ & $\begin{array}{c}-0.1606^{* * *} \\
(0.0340)\end{array}$ & $\begin{array}{c}-0.2173 * * * \\
(0.0646)\end{array}$ & $\begin{array}{c}-0.2252^{* * *} \\
(0.0499)\end{array}$ & $\begin{array}{c}-0.1853 * * * \\
(0.0365)\end{array}$ & $\begin{array}{c}-0.1876 * * * \\
(0.0321)\end{array}$ & $\begin{array}{c}-0.1838^{* * *} \\
(0.0537)\end{array}$ & $\begin{array}{c}-0.1895^{* * *} \\
(0.0401)\end{array}$ \\
\hline Gini & $\begin{array}{c}0.1841 \\
(0.1314)\end{array}$ & $\begin{array}{c}0.3553 \\
(0.6222)\end{array}$ & $\begin{array}{c}0.1722 \\
(0.1119)\end{array}$ & $\begin{array}{l}1.0257^{*} \\
(0.6037)\end{array}$ & $\begin{array}{l}0.2558^{*} \\
(0.1526)\end{array}$ & $\begin{array}{c}1.0467 \\
(0.6766)\end{array}$ & $\begin{array}{l}0.2741 * \\
(0.1614)\end{array}$ & $\begin{array}{c}0.7293 \\
(0.6367)\end{array}$ & $\begin{array}{c}0.1258 \\
(0.1109)\end{array}$ & $\begin{array}{c}0.6964 \\
(0.5975)\end{array}$ \\
\hline GDP per capita & $\begin{array}{l}-0.0000 \\
(0.0000)\end{array}$ & $\begin{array}{l}-0.0000 \\
(0.0000)\end{array}$ & $\begin{array}{c}-0.0000^{* *} \\
(0.0000)\end{array}$ & $\begin{array}{c}-0.0000^{* *} \\
(0.0000)\end{array}$ & $\begin{array}{l}-0.0000 \\
(0.0000)\end{array}$ & $\begin{array}{l}-0.0000 \\
(0.0000)\end{array}$ & $\begin{array}{c}-0.0000^{* *} \\
(0.0000)\end{array}$ & $\begin{array}{c}-0.0000^{* *} \\
(0.0000)\end{array}$ & $\begin{array}{l}-0.0000^{*} \\
(0.0000)\end{array}$ & $\begin{array}{l}-0.0000 \\
(0.0000)\end{array}$ \\
\hline Rural inequality & $\begin{array}{l}-0.0595 \\
(0.0553)\end{array}$ & $\begin{array}{l}-0.0549 \\
(0.0571)\end{array}$ & $\begin{array}{l}-0.0842 \\
(0.0574)\end{array}$ & $\begin{array}{l}-0.0617 \\
(0.0547)\end{array}$ & $\begin{array}{c}-0.1607 * * \\
(0.0643)\end{array}$ & $\begin{array}{c}-0.1392^{* *} \\
(0.0623)\end{array}$ & $\begin{array}{c}-0.1634 * * * \\
(0.0613)\end{array}$ & $\begin{array}{c}-0.1508^{* *} \\
(0.0589)\end{array}$ & $\begin{array}{c}-0.1145^{* *} \\
(0.0560)\end{array}$ & $\begin{array}{c}-0.0989 * \\
(0.0549)\end{array}$ \\
\hline Vanhanen Knowledge & $\begin{array}{l}-0.0017^{*} \\
(0.0010)\end{array}$ & $\begin{array}{l}-0.0016^{*} \\
(0.0009)\end{array}$ & $\begin{array}{l}-0.0014 \\
(0.0010)\end{array}$ & $\begin{array}{l}-0.0010 \\
(0.0009)\end{array}$ & $\begin{array}{l}-0.0018 \\
(0.0014)\end{array}$ & $\begin{array}{l}-0.0015 \\
(0.0010)\end{array}$ & $\begin{array}{l}-0.0017 \\
(0.0012)\end{array}$ & $\begin{array}{l}-0.0015 \\
(0.0009)\end{array}$ & $\begin{array}{l}-0.0014 \\
(0.0010)\end{array}$ & $\begin{array}{l}-0.0012 \\
(0.0009)\end{array}$ \\
\hline Year & $\begin{array}{c}0.0036 * * * \\
(0.0010)\end{array}$ & $\begin{array}{c}0.0036^{* * *} \\
(0.0008)\end{array}$ & $\begin{array}{c}0.0035^{* * *} \\
(0.0011)\end{array}$ & $\begin{array}{c}0.0035^{* * *} \\
(0.0008)\end{array}$ & $\begin{array}{c}0.0038^{* * *} \\
(0.0013)\end{array}$ & $\begin{array}{c}0.0038^{* * *} \\
(0.0009)\end{array}$ & $\begin{array}{c}0.0036^{* * *} \\
(0.0011)\end{array}$ & $\begin{array}{c}0.0036^{* * *} \\
(0.0008)\end{array}$ & $\begin{array}{c}0.0031 * * * \\
(0.0009)\end{array}$ & $\begin{array}{c}0.0031 * * * \\
(0.0008)\end{array}$ \\
\hline Constant & $\begin{array}{c}-6.8727 * * * \\
(1.9259)\end{array}$ & $\begin{array}{c}-6.9470^{* * *} \\
(1.6271)\end{array}$ & $\begin{array}{c}-6.7869 * * * \\
(2.0856)\end{array}$ & $\begin{array}{c}-7.1882^{* * *} \\
(1.5653)\end{array}$ & $\begin{array}{c}-7.38566^{* * *} \\
(2.5053)\end{array}$ & $\begin{array}{c}-7.7338^{* * *} \\
(1.7867)\end{array}$ & $\begin{array}{c}-7.0359 * * * \\
(2.0521)\end{array}$ & $\begin{array}{c}-7.2248 * * * \\
(1.6772)\end{array}$ & $\begin{array}{c}-5.9414 * * * \\
(1.7870)\end{array}$ & $\begin{array}{c}-6.1866 \text { *** } \\
(1.5599)\end{array}$ \\
\hline $\mathrm{N}$ & 3827 & 3826 & 3827 & 3826 & 3827 & 3826 & 3827 & 3826 & 3827 & 3826 \\
\hline R2 & 0.021 & & 0.018 & & 0.021 & & 0.023 & & 0.017 & \\
\hline Prob $>$ F & 0.000 & 0.000 & 0.000 & 0.000 & 0.000 & 0.000 & 0.000 & 0.000 & 0.000 & 0.000 \\
\hline
\end{tabular}




\begin{tabular}{|c|c|c|c|c|c|c|c|c|c|c|}
\hline & \multicolumn{2}{|c|}{$\begin{array}{l}\text { V-Dem: Electoral } \\
\text { Democracy (ordinal) }\end{array}$} & \multicolumn{2}{|c|}{$\begin{array}{l}\text { V-Dem: Liberal Democracy } \\
\text { (ordinal) }\end{array}$} & \multicolumn{2}{|c|}{$\begin{array}{l}\text { V-Dem: Participatory } \\
\text { Democracy (ordinal) }\end{array}$} & \multicolumn{2}{|c|}{$\begin{array}{l}\text { V-Dem: Deliberative } \\
\text { Democracy (ordinal) }\end{array}$} & \multicolumn{2}{|c|}{$\begin{array}{l}\text { V-Dem: Egalitarian } \\
\text { Democracy (ordinal) }\end{array}$} \\
\hline & T3, MC & T3, ME & T3, MC & T3, ME & T3, MC & T3, ME & T3, MC & T3, ME & T3, MC & T3, ME \\
\hline Lag Democracy Score & $\begin{array}{c}-0.1592^{* * *} \\
(0.0457)\end{array}$ & $\begin{array}{c}-0.1711 * * * \\
(0.0410)\end{array}$ & $\begin{array}{c}-0.1650 * * * \\
(0.0344)\end{array}$ & $\begin{array}{c}-0.1950 * * * \\
(0.0308)\end{array}$ & $\begin{array}{c}-0.2268^{* * *} \\
(0.0309)\end{array}$ & $\begin{array}{c}-0.2315^{* * *} \\
(0.0247)\end{array}$ & $\begin{array}{c}-0.1817 * * * \\
(0.0312)\end{array}$ & $\begin{array}{c}-0.1968 * * * \\
(0.0259)\end{array}$ & $\begin{array}{c}-0.2340^{* * *} \\
(0.0494)\end{array}$ & $\begin{array}{c}-0.2984 * * * \\
(0.0382)\end{array}$ \\
\hline Gini & $\begin{array}{l}-0.0128 \\
(0.1481)\end{array}$ & $\begin{array}{c}1.3387 \\
(1.2735)\end{array}$ & $\begin{array}{c}0.1459 \\
(0.1180)\end{array}$ & $\begin{array}{l}1.9318^{*} \\
(1.0581)\end{array}$ & $\begin{array}{l}-0.0038 \\
(0.0916)\end{array}$ & $\begin{array}{c}0.4101 \\
(0.4462)\end{array}$ & $\begin{array}{c}0.0729 \\
(0.1135)\end{array}$ & $\begin{array}{l}1.2144 * \\
(0.7197)\end{array}$ & $\begin{array}{c}0.1927 \\
(0.1325)\end{array}$ & $\begin{array}{c}4.0567 * * * \\
(1.1787)\end{array}$ \\
\hline GDP per capita & $\begin{array}{l}0.0000^{* *} \\
(0.0000)\end{array}$ & $\begin{array}{c}0.0000^{* * *} \\
(0.0000)\end{array}$ & $\begin{array}{c}0.0000 \\
(0.0000)\end{array}$ & $\begin{array}{c}0.0000^{* * *} \\
(0.0000)\end{array}$ & $\begin{array}{l}-0.0000 \\
(0.0000)\end{array}$ & $\begin{array}{l}-0.0000 \\
(0.0000)\end{array}$ & $\begin{array}{c}0.0000 \\
(0.0000)\end{array}$ & $\begin{array}{c}0.0000 \\
(0.0000)\end{array}$ & $\begin{array}{c}0.0000 \\
(0.0000)\end{array}$ & $\begin{array}{c}0.0000 \\
(0.0000)\end{array}$ \\
\hline Rural inequality & $\begin{array}{c}-0.1030^{*} \\
(0.0589)\end{array}$ & $\begin{array}{l}-0.0556 \\
(0.0758)\end{array}$ & $\begin{array}{l}-0.0407 \\
(0.0480)\end{array}$ & $\begin{array}{c}0.0185 \\
(0.0585)\end{array}$ & $\begin{array}{c}-0.1242^{* * * *} \\
(0.0393)\end{array}$ & $\begin{array}{c}-0.1132^{* * *} \\
(0.0409)\end{array}$ & $\begin{array}{c}-0.1024 * * \\
(0.0405)\end{array}$ & $\begin{array}{l}-0.0682 \\
(0.0512)\end{array}$ & $\begin{array}{c}-0.1305^{* * *} \\
(0.0465)\end{array}$ & $\begin{array}{c}0.0065 \\
(0.0667)\end{array}$ \\
\hline Vanhanen Knowledge & $\begin{array}{c}-0.0040 * * * \\
(0.0013)\end{array}$ & $\begin{array}{c}-0.0039 * * * \\
(0.0011)\end{array}$ & $\begin{array}{c}-0.0032 * * * \\
(0.0011)\end{array}$ & $\begin{array}{c}-0.0031 * * * \\
(0.0008)\end{array}$ & $\begin{array}{c}-0.0022^{* *} \\
(0.0008)\end{array}$ & $\begin{array}{c}-0.0020^{* * *} \\
(0.0007)\end{array}$ & $\begin{array}{l}-0.0020^{*} \\
(0.0011)\end{array}$ & $\begin{array}{c}-0.0017 * * \\
(0.0008)\end{array}$ & $\begin{array}{l}-0.0011 \\
(0.0013)\end{array}$ & $\begin{array}{l}-0.0004 \\
(0.0009)\end{array}$ \\
\hline Year & $\begin{array}{c}0.0037 * * * \\
(0.0011)\end{array}$ & $\begin{array}{c}0.0043 * * * \\
(0.0011)\end{array}$ & $\begin{array}{c}0.0035^{* * *} \\
(0.0009)\end{array}$ & $\begin{array}{c}0.0042^{* * *} \\
(0.0008)\end{array}$ & $\begin{array}{c}0.0029 * * * \\
(0.0009)\end{array}$ & $\begin{array}{c}0.0029 * * * \\
(0.0006)\end{array}$ & $\begin{array}{c}0.0037 * * * \\
(0.0009)\end{array}$ & $\begin{array}{c}0.0039 * * * \\
(0.0007)\end{array}$ & $\begin{array}{c}0.0021 * * \\
(0.0010)\end{array}$ & $\begin{array}{c}0.0036^{* * * *} \\
(0.0009)\end{array}$ \\
\hline Constant & $\begin{array}{c}-7.1192 * * * \\
(2.1354)\end{array}$ & $\begin{array}{c}-8.8979 * * * \\
(2.5548)\end{array}$ & $\begin{array}{c}-6.8657 * * * \\
(1.7991)\end{array}$ & $\begin{array}{c}-9.0016^{* * * *} \\
(1.9196)\end{array}$ & $\begin{array}{c}-5.5633^{* * * *} \\
(1.7972)\end{array}$ & $\begin{array}{c}-5.7648^{* * * *} \\
(1.1826)\end{array}$ & $\begin{array}{c}-7.1256^{* * *} \\
(1.6703)\end{array}$ & $\begin{array}{c}-8.0032^{* * *} \\
(1.5256)\end{array}$ & $\begin{array}{c}-3.9834^{* *} \\
(1.9928)\end{array}$ & $\begin{array}{c}-8.7642 * * * \\
(2.1572)\end{array}$ \\
\hline $\mathrm{N}$ & 2996 & 2995 & 3343 & 3342 & 3810 & 3809 & 3419 & 3418 & 3374 & 3373 \\
\hline R2 & 0.021 & & 0.029 & & 0.032 & & 0.036 & & 0.033 & \\
\hline Prob $>$ F & 0.000 & 0.000 & 0.000 & 0.000 & 0.000 & 0.000 & 0.000 & 0.000 & 0.000 & 0.000 \\
\hline
\end{tabular}




\begin{tabular}{|c|c|c|c|c|c|c|c|c|}
\hline & \multicolumn{2}{|c|}{$\begin{array}{l}\text { Freedom House (FIW, any } \\
\text { change) }\end{array}$} & \multicolumn{2}{|c|}{$\begin{array}{c}\text { Freedom House (PR, any } \\
\text { change) }\end{array}$} & \multicolumn{2}{|c|}{$\begin{array}{c}\text { Freedom House (FIW, 1+ } \\
\text { points) }\end{array}$} & \multicolumn{2}{|c|}{$\begin{array}{c}\text { Freedom House (PR, 1+ } \\
\text { points) }\end{array}$} \\
\hline & T3, MC & T3, ME & T3, MC & T3, ME & T3, MC & T3, ME & T3, MC & T3, ME \\
\hline Lag Democracy Score & $\begin{array}{c}0.0435^{* *} \\
(0.0205)\end{array}$ & $\begin{array}{c}0.1538 \\
(0.1008)\end{array}$ & $\begin{array}{c}0.0366^{* *} \\
(0.0149)\end{array}$ & $\begin{array}{c}0.0738^{* *} \\
(0.0302)\end{array}$ & $\begin{array}{l}-0.0070 \\
(0.0158)\end{array}$ & $\begin{array}{c}0.0324 \\
(0.0431)\end{array}$ & $\begin{array}{c}0.0346^{* *} \\
(0.0156)\end{array}$ & $\begin{array}{c}0.0784^{* *} \\
(0.0319)\end{array}$ \\
\hline Gini & $\begin{array}{l}-0.5464 \\
(0.8605)\end{array}$ & $\begin{array}{l}-57.9453 \\
(47.1835)\end{array}$ & $\begin{array}{l}-0.2816 \\
(0.6729)\end{array}$ & $\begin{array}{l}-18.7371 \\
(12.0208)\end{array}$ & $\begin{array}{c}0.7809 \\
(0.8408)\end{array}$ & $\begin{array}{l}-19.6845 \\
(20.1655)\end{array}$ & $\begin{array}{l}-0.2008 \\
(0.7344)\end{array}$ & $\begin{array}{l}-21.8923^{*} \\
(12.7030)\end{array}$ \\
\hline GDP per capita & $\begin{array}{l}-0.0000 \\
(0.0000)\end{array}$ & $\begin{array}{c}0.0002 \\
(0.0002)\end{array}$ & $\begin{array}{l}-0.0000 \\
(0.0000)\end{array}$ & $\begin{array}{c}0.0001 \\
(0.0000)\end{array}$ & $\begin{array}{l}-0.0000 \\
(0.0000)\end{array}$ & $\begin{array}{c}0.0001 \\
(0.0001)\end{array}$ & $\begin{array}{l}-0.0000 \\
(0.0000)\end{array}$ & $\begin{array}{c}0.0001 \\
(0.0001)\end{array}$ \\
\hline Rural inequality & $\begin{array}{l}-0.0029 \\
(0.1345)\end{array}$ & $\begin{array}{l}-0.2694 \\
(0.4421)\end{array}$ & $\begin{array}{c}0.0682 \\
(0.1119)\end{array}$ & $\begin{array}{c}0.0099 \\
(0.1852)\end{array}$ & $\begin{array}{l}-0.0225 \\
(0.0838)\end{array}$ & $\begin{array}{l}-0.1175 \\
(0.1889)\end{array}$ & $\begin{array}{c}0.0327 \\
(0.1111)\end{array}$ & $\begin{array}{l}-0.0359 \\
(0.1957)\end{array}$ \\
\hline Vanhanen Knowledge & $\begin{array}{l}-0.0077^{*} \\
(0.0046)\end{array}$ & $\begin{array}{l}-0.0051 \\
(0.0076)\end{array}$ & $\begin{array}{c}-0.0108^{* * * *} \\
(0.0037)\end{array}$ & $\begin{array}{c}-0.0094 * * * \\
(0.0034)\end{array}$ & $\begin{array}{c}-0.0051 * * \\
(0.0022)\end{array}$ & $\begin{array}{l}-0.0042 \\
(0.0032)\end{array}$ & $\begin{array}{c}-0.0105^{* * *} \\
(0.0035)\end{array}$ & $\begin{array}{c}-0.0089 * * \\
(0.0036)\end{array}$ \\
\hline Year & $\begin{array}{c}0.0129 * * * \\
(0.0035)\end{array}$ & $\begin{array}{c}0.0175^{* *} \\
(0.0083)\end{array}$ & $\begin{array}{c}0.0143^{* * *} \\
(0.0028)\end{array}$ & $\begin{array}{c}0.0163^{* * *} \\
(0.0037)\end{array}$ & $\begin{array}{c}0.0075^{* * *} \\
(0.0017)\end{array}$ & $\begin{array}{c}0.0092^{* * *} \\
(0.0035)\end{array}$ & $\begin{array}{c}0.0140^{* * *} \\
(0.0027)\end{array}$ & $\begin{array}{c}0.0163^{* * *} \\
(0.0039)\end{array}$ \\
\hline Constant & $\begin{array}{c}-25.0009 * * * \\
(6.8121)\end{array}$ & $\begin{array}{c}-9.2195 \\
(19.5188)\end{array}$ & $\begin{array}{c}-27.9867^{* * * *} \\
(5.4240)\end{array}$ & $\begin{array}{c}-23.8496 * * * \\
(7.3188)\end{array}$ & $\begin{array}{c}-14.9724 * * * \\
(3.4401)\end{array}$ & $\begin{array}{l}-9.3457 \\
(8.3420)\end{array}$ & $\begin{array}{c}-27.3934 * * * \\
(5.2670)\end{array}$ & $\begin{array}{c}-22.5308^{* * *} \\
(7.7341)\end{array}$ \\
\hline $\mathrm{N}$ & 1367 & 1367 & 1392 & 1392 & 1367 & 1367 & 1392 & 1392 \\
\hline $\mathrm{R} 2$ & 0.025 & & 0.033 & & 0.023 & & 0.036 & \\
\hline Prob $>$ F & 0.001 & 0.757 & 0.000 & 0.000 & 0.001 & 0.989 & 0.000 & 0.000 \\
\hline
\end{tabular}




\begin{tabular}{|c|c|c|c|c|c|c|c|c|c|}
\hline & Gasiorowski 1996 (hybrid) & \multicolumn{2}{|c|}{$\begin{array}{c}\text { Gates et al. } 2006 \text { (any } \\
\text { change) }\end{array}$} & \multicolumn{2}{|c|}{ Polity IV (any change) } & \multicolumn{2}{|c|}{ Polity IV (3+ points) } & \multicolumn{2}{|c|}{ Polity IV (5+ points) } \\
\hline & T3, ME & T3, MC & T3, ME & T3, MC & T3, ME & T3, MC & T3, ME & T3, MC & T3, ME \\
\hline Lag Democracy Score & insufficient observations & $\begin{array}{l}-0.1346 \\
(0.0869)\end{array}$ & $\begin{array}{c}-0.3643 * * \\
(0.1760)\end{array}$ & $\begin{array}{c}0.0012 \\
(0.0060)\end{array}$ & $\begin{array}{c}0.0011 \\
(0.0039)\end{array}$ & $\begin{array}{l}0.0098^{*} \\
(0.0057)\end{array}$ & $\begin{array}{c}0.0097 * * * \\
(0.0031)\end{array}$ & $\begin{array}{c}0.0073 \\
(0.0047)\end{array}$ & $\begin{array}{c}0.0073 * * * \\
(0.0027)\end{array}$ \\
\hline Gini & & $\begin{array}{c}0.6224 \\
(0.5716)\end{array}$ & $\begin{array}{l}27.4112^{*} \\
(15.2983)\end{array}$ & $\begin{array}{l}0.1550 \\
(0.3295)\end{array}$ & $\begin{array}{l}0.5567 \\
(0.7857)\end{array}$ & $\begin{array}{l}0.2528 \\
(0.2753)\end{array}$ & $\begin{array}{c}0.6489 \\
(0.6332)\end{array}$ & $\begin{array}{c}0.3001 \\
(0.2573)\end{array}$ & $\begin{array}{c}0.2805 \\
(0.5392)\end{array}$ \\
\hline GDP per capita & & $\begin{array}{l}-0.0000 \\
(0.0000)\end{array}$ & $\begin{array}{l}-0.0001 * \\
(0.0000)\end{array}$ & $\begin{array}{l}0.0000 \\
(0.0000)\end{array}$ & $\begin{array}{l}0.0000^{*} \\
(0.0000)\end{array}$ & $\begin{array}{l}0.0000^{*} \\
(0.0000)\end{array}$ & $\begin{array}{l}0.0000^{* *} \\
(0.0000)\end{array}$ & $\begin{array}{l}0.0000^{*} \\
(0.0000)\end{array}$ & $\begin{array}{l}0.0000^{* *} \\
(0.0000)\end{array}$ \\
\hline Rural inequality & & $\begin{array}{c}-0.1140 * * * \\
(0.0426)\end{array}$ & $\begin{array}{l}0.4461 \\
(0.3476)\end{array}$ & $\begin{array}{l}-0.1102 \\
(0.1034)\end{array}$ & $\begin{array}{l}-0.0889 \\
(0.0951)\end{array}$ & $\begin{array}{l}-0.0886 \\
(0.0686)\end{array}$ & $\begin{array}{l}-0.0676 \\
(0.0766)\end{array}$ & $\begin{array}{l}-0.0086 \\
(0.0692)\end{array}$ & $\begin{array}{l}-0.0096 \\
(0.0653)\end{array}$ \\
\hline Vanhanen Knowledge & & $\begin{array}{c}-0.0061 * * * \\
(0.0016)\end{array}$ & $\begin{array}{c}0.0048 \\
(0.0070)\end{array}$ & $\begin{array}{l}-0.0044^{*} \\
(0.0022)\end{array}$ & $\begin{array}{c}-0.0042^{* *} \\
(0.0019)\end{array}$ & $\begin{array}{c}-0.0045^{* *} \\
(0.0017)\end{array}$ & $\begin{array}{c}-0.0044^{* * *} \\
(0.0015)\end{array}$ & $\begin{array}{c}-0.0055^{* * *} \\
(0.0017)\end{array}$ & $\begin{array}{c}-0.0055^{* * *} \\
(0.0013)\end{array}$ \\
\hline Year & & $\begin{array}{c}0.0071^{* * *} \\
(0.0013)\end{array}$ & $\begin{array}{c}0.0098^{* * *} \\
(0.0031)\end{array}$ & $\begin{array}{c}0.0059 * * \\
(0.0026)\end{array}$ & $\begin{array}{c}0.0061^{* * *} \\
(0.0017)\end{array}$ & $\begin{array}{l}0.0046^{* *} \\
(0.0019)\end{array}$ & $\begin{array}{c}0.0047 * * * \\
(0.0014)\end{array}$ & $\begin{array}{c}0.0051 * * * \\
(0.0017)\end{array}$ & $\begin{array}{c}0.0051 * * * \\
(0.0012)\end{array}$ \\
\hline Constant & & $\begin{array}{c}-13.9160^{* * *} \\
(2.5341)\end{array}$ & $\begin{array}{c}-31.6344^{* * *} \\
(11.4297)\end{array}$ & $\begin{array}{c}-11.5352^{* *} \\
(5.1221)\end{array}$ & $\begin{array}{c}-12.0600^{* * *} \\
(3.4454)\end{array}$ & $\begin{array}{c}-8.9028^{* *} \\
(3.7374)\end{array}$ & $\begin{array}{c}-9.4202^{* * *} \\
(2.7768)\end{array}$ & $\begin{array}{c}-9.9251 * * * \\
(3.3390)\end{array}$ & $\begin{array}{c}-9.8996 * * * \\
(2.3644)\end{array}$ \\
\hline $\begin{array}{l}\mathrm{N} \\
\mathrm{R} 2\end{array}$ & & 1843 & 1843 & 1904 & 1904 & 1904 & 1904 & 1904 & 1904 \\
\hline $\begin{array}{l}\text { R2 } \\
\text { Prob > F }\end{array}$ & & $\begin{array}{l}0.030 \\
0.000\end{array}$ & 1.000 & $\begin{array}{l}0.030 \\
0.000\end{array}$ & 0.000 & $\begin{array}{l}0.036 \\
0.000\end{array}$ & 0.000 & $\begin{array}{l}0.033 \\
0.001\end{array}$ & 0.000 \\
\hline
\end{tabular}




\begin{tabular}{|c|c|c|c|c|c|c|}
\hline & Reich 2002 (hybrid) & Skaaning et al. 2015 & \multicolumn{2}{|c|}{$\begin{array}{c}\text { Wahman et al. } 2013 \text { (any } \\
\text { change) }\end{array}$} & \multicolumn{2}{|c|}{$\begin{array}{c}\text { Wahman et al. } 2013 \text { (1.5+ } \\
\text { points) }\end{array}$} \\
\hline & T3, MC & T3, ME & T3, MC & T3, ME & T3, MC & T3, ME \\
\hline Lag Democracy Score & insufficient observations & no DEM/ AUT threshold & $\begin{array}{l}-0.0182 \\
(0.0136)\end{array}$ & $\begin{array}{c}-0.0490^{* *} \\
(0.0229)\end{array}$ & $\begin{array}{c}0.0006 \\
(0.0111)\end{array}$ & $\begin{array}{l}-0.0121 \\
(0.0115)\end{array}$ \\
\hline Gini & & & $\begin{array}{l}-0.6576 \\
(0.5983)\end{array}$ & $\begin{array}{c}-25.3300^{*} \\
(14.4428)\end{array}$ & $\begin{array}{c}1.0121^{* *} \\
(0.4820)\end{array}$ & $\begin{array}{l}-9.1285 \\
(7.2471)\end{array}$ \\
\hline GDP per capita & & & $\begin{array}{l}-0.0000 \\
(0.0000)\end{array}$ & $\begin{array}{l}-0.0000 \\
(0.0000)\end{array}$ & $\begin{array}{c}0.0000 \\
(0.0000)\end{array}$ & $\begin{array}{c}0.0000 \\
(0.0000)\end{array}$ \\
\hline Rural inequality & & & $\begin{array}{l}-0.1118 \\
(0.1405)\end{array}$ & $\begin{array}{l}-0.2400 \\
(0.2427)\end{array}$ & $\begin{array}{l}-0.0455 \\
(0.0953)\end{array}$ & $\begin{array}{l}-0.0982 \\
(0.1218)\end{array}$ \\
\hline Vanhanen Knowledge & & & $\begin{array}{c}-0.0137 * * * \\
(0.0048)\end{array}$ & $\begin{array}{c}-0.0115^{* * *} \\
(0.0044)\end{array}$ & $\begin{array}{c}-0.0092^{* * *} \\
(0.0027)\end{array}$ & $\begin{array}{c}-0.0084 * * * \\
(0.0022)\end{array}$ \\
\hline Year & & & $\begin{array}{c}0.0179 * * * \\
(0.0036)\end{array}$ & $\begin{array}{c}0.0206 * * * \\
(0.0047)\end{array}$ & $\begin{array}{c}0.0087 * * * \\
(0.0018)\end{array}$ & $\begin{array}{c}0.0098^{* * *} \\
(0.0024)\end{array}$ \\
\hline Constant & & & $\begin{array}{c}-34.3658^{* * * *} \\
(7.0280)\end{array}$ & $\begin{array}{c}-28.4234 * * * \\
(9.4961)\end{array}$ & $\begin{array}{c}-17.3684 * * * \\
(3.4188)\end{array}$ & $\begin{array}{c}-14.9260^{* * *} \\
(4.7650)\end{array}$ \\
\hline $\mathrm{N}$ & & & 1500 & 1500 & 1500 & 1500 \\
\hline R2 & & & 0.041 & & 0.037 & \\
\hline Prob $>$ F & & & 0.000 & 0.000 & 0.000 & 0.114 \\
\hline
\end{tabular}




\section{Appendix XI: Robustness Checks: Gibler and Randazzo 2011}

a) all potential reformers

\begin{tabular}{|c|c|c|c|c|}
\hline & $\begin{array}{l}\text { Freedom } \\
\text { House } \\
\text { (FIW, any } \\
\text { change) }\end{array}$ & $\begin{array}{c}\text { Freedom } \\
\text { House (PR, } \\
\text { any change) }\end{array}$ & $\begin{array}{c}\text { Freedom } \\
\text { House } \\
\text { (FIW, 1+ } \\
\text { points) } \\
\end{array}$ & $\begin{array}{c}\text { Freedom } \\
\text { House (PR, } \\
1+\text { points) }\end{array}$ \\
\hline Established Judiciary & $\begin{array}{c}-0.506^{* *} \\
(0.20)\end{array}$ & $\begin{array}{c}-0.777 * * * \\
(0.28)\end{array}$ & $\begin{array}{c}-1.155^{* *} \\
(0.50)\end{array}$ & $\begin{array}{c}-0.721 * * * \\
(0.28)\end{array}$ \\
\hline Newly Formed Judiciary & $\begin{array}{l}0.269 \\
(0.26)\end{array}$ & $\begin{array}{c}0.586^{* *} \\
(0.29)\end{array}$ & $\begin{array}{c}0.749 * \\
(0.42)\end{array}$ & $\begin{array}{c}0.636^{* *} \\
(0.29)\end{array}$ \\
\hline Independent Legislature & $\begin{array}{c}-0.018 \\
(0.13)\end{array}$ & $\begin{array}{c}-0.349 * * \\
(0.16)\end{array}$ & $\begin{array}{c}-0.474 * * \\
(0.24)\end{array}$ & $\begin{array}{c}-0.375^{* *} \\
(0.16)\end{array}$ \\
\hline Wealth (log of GDP) & $\begin{array}{c}-0.209 * * * \\
(0.07)\end{array}$ & $\begin{array}{c}-0.257^{* * *} \\
(0.08)\end{array}$ & $\begin{array}{c}-0.379 * * * \\
(0.13)\end{array}$ & $\begin{array}{c}-0.220^{* * *} \\
(0.08)\end{array}$ \\
\hline Age of Democracy (logged) & $\begin{array}{c}-0.011^{* * *} \\
(0.00)\end{array}$ & $\begin{array}{r}-0.009 \\
(0.01)\end{array}$ & $\begin{array}{c}-0.002 \\
(0.01)\end{array}$ & $\begin{array}{c}-0.011 * \\
(0.01)\end{array}$ \\
\hline Past Democratic Reversions & $\begin{array}{l}0.169 \\
(0.12)\end{array}$ & $\begin{array}{l}0.227 \\
(0.15)\end{array}$ & $\begin{array}{l}0.333 \\
(0.23)\end{array}$ & $\begin{array}{l}0.217 \\
(0.15)\end{array}$ \\
\hline Economic Crisis & $\begin{array}{c}-0.142 \\
(0.12)\end{array}$ & $\begin{array}{l}0.013 \\
(0.15)\end{array}$ & $\begin{array}{c}-0.086 \\
(0.23)\end{array}$ & $\begin{array}{l}0.030 \\
(0.15)\end{array}$ \\
\hline Presence of Territorial Rivalry & $\begin{array}{l}0.135 \\
(0.14)\end{array}$ & $\begin{array}{l}0.266 \\
(0.17)\end{array}$ & $\begin{array}{c}0.607^{* *} \\
(0.25)\end{array}$ & $\begin{array}{c}0.301 * \\
(0.17)\end{array}$ \\
\hline Highest level of militarization among neighbors & $\begin{array}{l}0.124 \\
(0.13)\end{array}$ & $\begin{array}{l}0.018 \\
(0.16)\end{array}$ & $\begin{array}{c}-0.043 \\
(0.25)\end{array}$ & $\begin{array}{c}-0.036 \\
(0.17)\end{array}$ \\
\hline Constant & $\begin{array}{l}0.450 \\
(0.86)\end{array}$ & $\begin{array}{l}0.071 \\
(1.07)\end{array}$ & $\begin{array}{c}-0.235 \\
(1.65)\end{array}$ & $\begin{array}{c}-0.494 \\
(1.09)\end{array}$ \\
\hline $\mathrm{N}$ & 3022 & 2739 & 2744 & 2736 \\
\hline Chi2 & 94.794 & 104.639 & 64.370 & 97.659 \\
\hline Prob $>$ Chi2 & 0.000 & 0.000 & 0.000 & 0.000 \\
\hline Pseudo-R2 & 0.043 & 0.067 & 0.081 & 0.065 \\
\hline
\end{tabular}




\begin{tabular}{|c|c|c|c|c|c|}
\hline & $\begin{array}{c}\text { Gasiorowski } \\
1996 \\
\text { (hybrid) }\end{array}$ & $\begin{array}{c}\text { Gates et al. } \\
2006 \text { (any } \\
\text { change) }\end{array}$ & $\begin{array}{c}\text { Polity IV } \\
\text { (any } \\
\text { change) }\end{array}$ & $\begin{array}{c}\text { Polity IV } \\
(3+\text { points })\end{array}$ & $\begin{array}{c}\text { Polity IV } \\
(5+\text { points })\end{array}$ \\
\hline Established Judiciary & $\begin{array}{l}-0.427 \\
(1.25)\end{array}$ & $\begin{array}{c}-1.138^{* *} \\
(0.50)\end{array}$ & $\begin{array}{c}-1.564 * * * \\
(0.48)\end{array}$ & $\begin{array}{c}-3.255^{* * *} \\
(1.20)\end{array}$ & $\begin{array}{c}0.000 \\
(.)\end{array}$ \\
\hline Newly Formed Judiciary & $\begin{array}{l}1.387 \\
(1.13)\end{array}$ & $\begin{array}{l}0.440 \\
(0.44)\end{array}$ & $\begin{array}{c}0.642^{*} \\
(0.38)\end{array}$ & $\begin{array}{l}0.521 \\
(0.60)\end{array}$ & $\begin{array}{l}0.349 \\
(0.71)\end{array}$ \\
\hline Independent Legislature & $\begin{array}{c}-0.969 * \\
(0.59)\end{array}$ & $\begin{array}{c}-1.074 * * * \\
(0.22)\end{array}$ & $\begin{array}{c}-0.211 \\
(0.20)\end{array}$ & $\begin{array}{c}-1.086^{* * *} \\
(0.30)\end{array}$ & $\begin{array}{c}-2.374 * * * \\
(0.36)\end{array}$ \\
\hline Wealth (log of GDP) & $\begin{array}{l}0.035 \\
(0.28)\end{array}$ & $\begin{array}{c}-0.332 * * * \\
(0.12)\end{array}$ & $\begin{array}{c}-0.195^{*} \\
(0.11)\end{array}$ & $\begin{array}{c}-0.216 \\
(0.19)\end{array}$ & $\begin{array}{c}-0.485^{* *} \\
(0.23)\end{array}$ \\
\hline Age of Democracy (logged) & $\begin{array}{l}0.018 \\
(0.02)\end{array}$ & $\begin{array}{c}-0.006 \\
(0.01)\end{array}$ & $\begin{array}{l}0.004 \\
(0.01)\end{array}$ & $\begin{array}{l}0.014 \\
(0.01)\end{array}$ & $\begin{array}{l}0.012 \\
(0.01)\end{array}$ \\
\hline Past Democratic Reversions & $\begin{array}{c}1.241 * * \\
(0.50)\end{array}$ & $\begin{array}{c}0.465^{* *} \\
(0.21)\end{array}$ & $\begin{array}{c}0.501^{* * *} \\
(0.19)\end{array}$ & $\begin{array}{l}0.502^{*} \\
(0.28)\end{array}$ & $\begin{array}{l}0.032 \\
(0.34)\end{array}$ \\
\hline Economic Crisis & $\begin{array}{c}-0.114 \\
(0.46)\end{array}$ & $\begin{array}{l}-0.247 \\
(0.21)\end{array}$ & $\begin{array}{l}0.152 \\
(0.19)\end{array}$ & $\begin{array}{l}-0.235 \\
(0.28)\end{array}$ & $\begin{array}{l}-0.157 \\
(0.33)\end{array}$ \\
\hline Presence of Territorial Rivalry & $\begin{array}{c}1.061 * * \\
(0.48)\end{array}$ & $\begin{array}{c}0.559 * * \\
(0.23)\end{array}$ & $\begin{array}{l}0.295 \\
(0.20)\end{array}$ & $\begin{array}{l}0.063 \\
(0.30)\end{array}$ & $\begin{array}{l}0.372 \\
(0.34)\end{array}$ \\
\hline Highest level of militarization among neighbors & $\begin{array}{c}-0.655 \\
(0.69)\end{array}$ & $\begin{array}{c}-0.062 \\
(0.24)\end{array}$ & $\begin{array}{l}-0.009 \\
(0.21)\end{array}$ & $\begin{array}{l}0.229 \\
(0.32)\end{array}$ & $\begin{array}{c}0.837 * * \\
(0.34)\end{array}$ \\
\hline Constant & $\begin{array}{c}-8.294^{* *} \\
(3.66)\end{array}$ & $\begin{array}{l}-0.169 \\
(1.49)\end{array}$ & $\begin{array}{l}-1.946 \\
(1.34)\end{array}$ & $\begin{array}{l}-0.644 \\
(2.09)\end{array}$ & $\begin{array}{c}5.357^{* *} \\
(2.51)\end{array}$ \\
\hline $\mathrm{N}$ & 2160 & 3192 & 3932 & 3325 & 1280 \\
\hline Chi2 & 20.306 & 104.659 & 61.928 & 62.313 & 70.125 \\
\hline Prob $>$ Chi2 & 0.016 & 0.000 & 0.000 & 0.000 & 0.000 \\
\hline Pseudo-R2 & 0.089 & 0.109 & 0.055 & 0.104 & 0.171 \\
\hline
\end{tabular}




\begin{tabular}{|c|c|c|c|c|}
\hline & $\begin{array}{c}\text { Reich } 2002 \\
\text { (hybrid) }\end{array}$ & $\begin{array}{l}\text { Skaaning et } \\
\text { al. } 2015\end{array}$ & $\begin{array}{c}\text { Wahman et } \\
\text { al. } 2013 \text { (any } \\
\text { change) }\end{array}$ & $\begin{array}{c}\text { Wahman et } \\
\text { al. } 2013 \\
\text { (1.5+ points) }\end{array}$ \\
\hline \multirow[t]{2}{*}{ Established Judiciary } & -0.410 & $-2.842^{* *}$ & $-0.469 * *$ & -0.979 \\
\hline & $(0.92)$ & $(1.12)$ & $(0.20)$ & $(0.77)$ \\
\hline \multirow[t]{2}{*}{ Newly Formed Judiciary } & 0.150 & 0.483 & 0.306 & $1.433^{* *}$ \\
\hline & $(1.06)$ & $(0.56)$ & $(0.25)$ & $(0.59)$ \\
\hline \multirow[t]{2}{*}{ Independent Legislature } & -0.581 & $-1.624 * * *$ & 0.173 & $-0.960 * *$ \\
\hline & $(0.47)$ & $(0.25)$ & $(0.13)$ & $(0.40)$ \\
\hline \multirow[t]{2}{*}{ Wealth (log of GDP) } & -0.119 & $-0.302 * *$ & $-0.130 * *$ & -0.231 \\
\hline & $(0.25)$ & $(0.14)$ & $(0.06)$ & $(0.20)$ \\
\hline \multirow[t]{2}{*}{ Age of Democracy (logged) } & -0.011 & 0.005 & $-0.013 * * *$ & 0.007 \\
\hline & $(0.02)$ & $(0.01)$ & $(0.00)$ & $(0.01)$ \\
\hline \multirow[t]{2}{*}{ Past Democratic Reversions } & $1.244 * * *$ & $0.603^{* * *}$ & $0.206^{*}$ & $0.679 *$ \\
\hline & $(0.43)$ & $(0.21)$ & $(0.12)$ & $(0.35)$ \\
\hline \multirow[t]{2}{*}{ Economic Crisis } & -0.209 & -0.146 & -0.159 & 0.084 \\
\hline & $(0.41)$ & $(0.21)$ & $(0.12)$ & $(0.36)$ \\
\hline \multirow[t]{2}{*}{ Presence of Territorial Rivalry } & $1.004 * *$ & $0.411^{*}$ & 0.145 & $0.690 *$ \\
\hline & $(0.41)$ & $(0.24)$ & $(0.13)$ & $(0.37)$ \\
\hline \multirow[t]{2}{*}{ Highest level of militarization among neighbors } & -0.785 & $0.405^{*}$ & -0.044 & -0.404 \\
\hline & $(0.56)$ & $(0.24)$ & $(0.13)$ & $(0.43)$ \\
\hline \multirow[t]{2}{*}{ Constant } & $-7.583 * *$ & 1.421 & -1.077 & -4.137 \\
\hline & $(3.22)$ & $(1.61)$ & $(0.83)$ & $(2.70)$ \\
\hline $\mathrm{N}$ & 3470 & 3394 & 3258 & 2884 \\
\hline Chi2 & 29.826 & 155.918 & 76.356 & 32.257 \\
\hline Prob $>$ Chi2 & 0.000 & 0.000 & 0.000 & 0.000 \\
\hline Pseudo-R2 & 0.097 & 0.162 & 0.033 & 0.081 \\
\hline
\end{tabular}




\begin{tabular}{|c|c|c|c|c|c|}
\hline & $\begin{array}{c}\text { V-Dem: } \\
\text { Electoral } \\
\text { Democracy }\end{array}$ & $\begin{array}{c}\text { V-Dem: } \\
\text { Liberal } \\
\text { Democracy }\end{array}$ & $\begin{array}{c}\text { V-Dem: } \\
\text { Participatory } \\
\text { Democracy }\end{array}$ & $\begin{array}{c}\text { V-Dem: } \\
\text { Deliberative } \\
\text { Democracy }\end{array}$ & $\begin{array}{c}\text { V-Dem: } \\
\text { Egalitarian } \\
\text { Democracy }\end{array}$ \\
\hline Established Judiciary & $\begin{array}{c}-0.774 \\
(0.49)\end{array}$ & $\begin{array}{c}-0.724 \\
(0.44)\end{array}$ & $\begin{array}{c}-0.043 \\
(0.33)\end{array}$ & $\begin{array}{c}-0.476 \\
(0.39)\end{array}$ & $\begin{array}{c}-0.732 \\
(0.45)\end{array}$ \\
\hline Newly Formed Judiciary & $\begin{array}{l}0.512 \\
(0.49)\end{array}$ & $\begin{array}{c}0.741^{*} \\
(0.42)\end{array}$ & $\begin{array}{l}0.387 \\
(0.41)\end{array}$ & $\begin{array}{l}0.547 \\
(0.42)\end{array}$ & $\begin{array}{l}0.614 \\
(0.45)\end{array}$ \\
\hline Independent Legislature & $\begin{array}{c}-0.851 * * * \\
(0.23)\end{array}$ & $\begin{array}{c}-0.521^{* *} \\
(0.23)\end{array}$ & $\begin{array}{c}-0.548^{* *} \\
(0.22)\end{array}$ & $\begin{array}{c}-1.309 * * * \\
(0.24)\end{array}$ & $\begin{array}{c}-0.450^{*} \\
(0.24)\end{array}$ \\
\hline Wealth (log of GDP) & $\begin{array}{c}-0.196 \\
(0.13)\end{array}$ & $\begin{array}{c}-0.240^{*} \\
(0.13)\end{array}$ & $\begin{array}{c}-0.009 \\
(0.13)\end{array}$ & $\begin{array}{c}-0.178 \\
(0.14)\end{array}$ & $\begin{array}{c}-0.194 \\
(0.13)\end{array}$ \\
\hline Age of Democracy (logged) & $\begin{array}{c}-0.004 \\
(0.01)\end{array}$ & $\begin{array}{c}-0.000 \\
(0.01)\end{array}$ & $\begin{array}{l}0.003 \\
(0.00)\end{array}$ & $\begin{array}{c}-0.000 \\
(0.01)\end{array}$ & $\begin{array}{l}0.001 \\
(0.01)\end{array}$ \\
\hline Past Democratic Reversions & $\begin{array}{c}0.763^{* * *} * \\
(0.20)\end{array}$ & $\begin{array}{c}0.762^{* * * *} \\
(0.20)\end{array}$ & $\begin{array}{c}0.994^{* * *} \\
(0.19)\end{array}$ & $\begin{array}{c}1.019^{* * *} \\
(0.21)\end{array}$ & $\begin{array}{c}0.758^{* * * *} \\
(0.21)\end{array}$ \\
\hline Economic Crisis & $\begin{array}{c}-0.019 \\
(0.20)\end{array}$ & $\begin{array}{r}-0.061 \\
(0.21)\end{array}$ & $\begin{array}{l}0.028 \\
(0.20)\end{array}$ & $\begin{array}{c}-0.125 \\
(0.22)\end{array}$ & $\begin{array}{l}-0.020 \\
(0.22)\end{array}$ \\
\hline Presence of Territorial Rivalry & $\begin{array}{c}0.368^{*} \\
(0.21)\end{array}$ & $\begin{array}{c}0.505^{* *} \\
(0.22)\end{array}$ & $\begin{array}{l}0.368^{*} \\
(0.21)\end{array}$ & $\begin{array}{c}0.452^{* *} \\
(0.22)\end{array}$ & $\begin{array}{c}0.399 * \\
(0.22)\end{array}$ \\
\hline Highest level of militarization among neighbors & $\begin{array}{c}-0.175 \\
(0.23)\end{array}$ & $\begin{array}{c}-0.401 \\
(0.25)\end{array}$ & $\begin{array}{c}-0.240 \\
(0.21)\end{array}$ & $\begin{array}{c}-0.130 \\
(0.23)\end{array}$ & $\begin{array}{l}-0.170 \\
(0.24)\end{array}$ \\
\hline Constant & $\begin{array}{c}-2.481 * \\
(1.45)\end{array}$ & $\begin{array}{c}-3.335^{* *} \\
(1.56)\end{array}$ & $\begin{array}{c}-4.544 * * * \\
(1.42)\end{array}$ & $\begin{array}{l}-1.891 \\
(1.60)\end{array}$ & $\begin{array}{c}-2.875^{*} \\
(1.55)\end{array}$ \\
\hline $\mathrm{N}$ & 3894 & 3868 & 3817 & 2952 & 3941 \\
\hline Chi2 & 82.664 & 63.167 & 45.424 & 100.538 & 47.207 \\
\hline Prob $>$ Chi2 & 0.000 & 0.000 & 0.000 & 0.000 & 0.000 \\
\hline Pseudo-R2 & 0.078 & 0.065 & 0.042 & 0.110 & 0.051 \\
\hline
\end{tabular}




\begin{tabular}{|c|c|c|c|c|c|}
\hline & $\begin{array}{c}\text { V-Dem: } \\
\text { Electoral } \\
\text { Democracy } \\
\text { (ordinal) }\end{array}$ & $\begin{array}{c}\text { V-Dem: } \\
\text { Liberal } \\
\text { Democracy } \\
\text { (ordinal) }\end{array}$ & $\begin{array}{c}\text { V-Dem: } \\
\text { Participatory } \\
\text { Democracy } \\
\text { (ordinal) }\end{array}$ & $\begin{array}{c}\text { V-Dem: } \\
\text { Deliberative } \\
\text { Democracy } \\
\text { (ordinal) }\end{array}$ & $\begin{array}{c}\text { V-Dem: } \\
\text { Egalitarian } \\
\text { Democracy } \\
\text { (ordinal) }\end{array}$ \\
\hline \multirow[t]{2}{*}{ Established Judiciary } & $-1.228 * *$ & -0.400 & $-1.422^{* *}$ & -0.651 & -0.382 \\
\hline & $(0.54)$ & $(0.49)$ & $(0.72)$ & $(0.41)$ & $(0.45)$ \\
\hline \multirow[t]{2}{*}{ Newly Formed Judiciary } & 0.292 & -0.027 & -0.140 & 0.105 & -0.580 \\
\hline & $(0.49)$ & $(0.63)$ & $(0.77)$ & $(0.49)$ & $(0.74)$ \\
\hline \multirow[t]{2}{*}{ Independent Legislature } & $-1.983^{* * *}$ & $-3.248 * * *$ & $-3.213 * * *$ & $-3.059 * * *$ & $-1.583 * * *$ \\
\hline & $(0.24)$ & $(0.36)$ & $(0.45)$ & $(0.36)$ & $(0.33)$ \\
\hline \multirow[t]{2}{*}{ Wealth (log of GDP) } & $-0.251 *$ & -0.274 & -0.089 & -0.088 & -0.188 \\
\hline & $(0.14)$ & $(0.18)$ & $(0.23)$ & $(0.17)$ & $(0.18)$ \\
\hline \multirow[t]{2}{*}{ Age of Democracy (logged) } & -0.011 & 0.001 & 0.004 & 0.001 & -0.013 \\
\hline & $(0.01)$ & $(0.01)$ & $(0.01)$ & $(0.01)$ & $(0.01)$ \\
\hline \multirow[t]{2}{*}{ Past Democratic Reversions } & $0.691 * * *$ & 0.183 & 0.353 & 0.293 & $0.643^{* *}$ \\
\hline & $(0.21)$ & $(0.32)$ & $(0.43)$ & $(0.31)$ & $(0.29)$ \\
\hline \multirow[t]{2}{*}{ Economic Crisis } & $-0.403 *$ & -0.173 & 0.398 & 0.048 & -0.115 \\
\hline & $(0.22)$ & $(0.32)$ & $(0.39)$ & $(0.30)$ & $(0.31)$ \\
\hline \multirow[t]{2}{*}{ Presence of Territorial Rivalry } & $0.398^{*}$ & $0.586^{*}$ & $1.174 * * *$ & $0.576^{*}$ & 0.220 \\
\hline & $(0.22)$ & $(0.33)$ & $(0.43)$ & $(0.33)$ & $(0.32)$ \\
\hline \multirow[t]{2}{*}{ Highest level of militarization among neighbors } & 0.357 & 0.116 & 0.583 & -0.009 & $0.546^{*}$ \\
\hline & $(0.24)$ & $(0.35)$ & $(0.38)$ & $(0.30)$ & $(0.31)$ \\
\hline \multirow[t]{2}{*}{ Constant } & 1.828 & 1.917 & 1.632 & 0.049 & 1.611 \\
\hline & $(1.61)$ & $(2.16)$ & $(2.73)$ & $(2.05)$ & $(2.14)$ \\
\hline $\mathrm{N}$ & 2634 & 1990 & 1840 & 1855 & 2137 \\
\hline Chi2 & 208.077 & 140.108 & 102.981 & 105.049 & 72.241 \\
\hline Prob $>$ Chi2 & 0.000 & 0.000 & 0.000 & 0.000 & 0.000 \\
\hline Pseudo-R2 & 0.220 & 0.264 & 0.278 & 0.186 & 0.136 \\
\hline
\end{tabular}


b) DEM only

\begin{tabular}{|c|c|c|c|c|}
\hline & $\begin{array}{c}\text { Freedom } \\
\text { House } \\
\text { (FIW, any } \\
\text { change) } \\
\end{array}$ & $\begin{array}{c}\text { Freedom } \\
\text { House (PR, } \\
\text { any change) }\end{array}$ & $\begin{array}{l}\text { Freedom } \\
\text { House } \\
\text { (FIW, 1+ } \\
\text { points) } \\
\end{array}$ & $\begin{array}{c}\text { Freedom } \\
\text { House (PR, } \\
1+\text { points) }\end{array}$ \\
\hline Established Judiciary & $\begin{array}{c}-0.530^{* *} \\
(0.25)\end{array}$ & $\begin{array}{c}-0.990 * * * \\
(0.34)\end{array}$ & $\begin{array}{c}-0.724 \\
(0.52)\end{array}$ & $\begin{array}{c}-0.895^{* * *} \\
(0.34)\end{array}$ \\
\hline Newly Formed Judiciary & $\begin{array}{l}0.385 \\
(0.32)\end{array}$ & $\begin{array}{c}0.683^{*} \\
(0.35)\end{array}$ & $\begin{array}{l}0.672 \\
(0.53)\end{array}$ & $\begin{array}{c}0.744^{* *} \\
(0.36)\end{array}$ \\
\hline Independent Legislature & $\begin{array}{c}-2.164 * * * \\
(0.46)\end{array}$ & $\begin{array}{c}-2.662 * * * \\
(0.52)\end{array}$ & $\begin{array}{c}-3.328^{* * *} \\
(0.51)\end{array}$ & $\begin{array}{c}-2.728^{* * *} \\
(0.52)\end{array}$ \\
\hline Wealth (log of GDP) & $\begin{array}{c}-0.422 * * * \\
(0.13)\end{array}$ & $\begin{array}{c}-0.295^{*} \\
(0.16)\end{array}$ & $\begin{array}{c}-0.533^{* *} \\
(0.22)\end{array}$ & $\begin{array}{c}-0.288^{*} \\
(0.16)\end{array}$ \\
\hline Age of Democracy (logged) & $\begin{array}{c}-0.013^{* *} \\
(0.01)\end{array}$ & $\begin{array}{c}-0.015^{*} \\
(0.01)\end{array}$ & $\begin{array}{l}-0.015 \\
(0.01)\end{array}$ & $\begin{array}{c}-0.016^{* *} \\
(0.01)\end{array}$ \\
\hline Past Democratic Reversions & $\begin{array}{l}0.050 \\
(0.21)\end{array}$ & $\begin{array}{l}0.217 \\
(0.27)\end{array}$ & $\begin{array}{l}0.331 \\
(0.39)\end{array}$ & $\begin{array}{l}0.296 \\
(0.27)\end{array}$ \\
\hline Economic Crisis & $\begin{array}{r}-0.241 \\
(0.20)\end{array}$ & $\begin{array}{l}-0.049 \\
(0.25)\end{array}$ & $\begin{array}{c}-0.066 \\
(0.36)\end{array}$ & $\begin{array}{r}-0.050 \\
(0.25)\end{array}$ \\
\hline Presence of Territorial Rivalry & $\begin{array}{l}0.324 \\
(0.23)\end{array}$ & $\begin{array}{l}0.093 \\
(0.30)\end{array}$ & $\begin{array}{c}0.797 * * \\
(0.39)\end{array}$ & $\begin{array}{l}0.138 \\
(0.30)\end{array}$ \\
\hline Highest level of militarization among neighbors & $\begin{array}{l}0.081 \\
(0.20)\end{array}$ & $\begin{array}{l}-0.022 \\
(0.25)\end{array}$ & $\begin{array}{c}-0.184 \\
(0.40)\end{array}$ & $\begin{array}{l}-0.034 \\
(0.25)\end{array}$ \\
\hline Constant & $\begin{array}{c}4.425^{* * *} \\
(1.57)\end{array}$ & $\begin{array}{l}2.932 \\
(1.90)\end{array}$ & $\begin{array}{l}3.512 \\
(2.79)\end{array}$ & $\begin{array}{l}2.772 \\
(1.93)\end{array}$ \\
\hline $\mathrm{N}$ & 1432 & 1399 & 1432 & 1399 \\
\hline Chi2 & 128.516 & 125.252 & 108.206 & 122.826 \\
\hline Prob $>$ Chi2 & 0.000 & 0.000 & 0.000 & 0.000 \\
\hline Pseudo-R2 & 0.133 & 0.179 & 0.266 & 0.181 \\
\hline
\end{tabular}




\begin{tabular}{|c|c|c|c|c|c|}
\hline & $\begin{array}{c}\text { Gasiorowski } \\
1996 \\
\text { (hybrid) }\end{array}$ & $\begin{array}{c}\text { Gates et al. } \\
2006 \text { (any } \\
\text { change) }\end{array}$ & $\begin{array}{c}\text { Polity IV } \\
\text { (any } \\
\text { change) }\end{array}$ & $\begin{array}{c}\text { Polity IV } \\
(3+\text { points })\end{array}$ & $\begin{array}{c}\text { Polity IV } \\
(5+\text { points })\end{array}$ \\
\hline \multirow[t]{2}{*}{ Established Judiciary } & 0.589 & $-1.066^{*}$ & & $-2.033^{*}$ & 0.000 \\
\hline & $(1.28)$ & $(0.59)$ & not concave & $(1.08)$ & $()$. \\
\hline \multirow[t]{2}{*}{ Newly Formed Judiciary } & 1.406 & 0.256 & & 0.435 & 0.445 \\
\hline & $(1.30)$ & $(0.55)$ & & $(0.64)$ & $(0.76)$ \\
\hline \multirow[t]{2}{*}{ Independent Legislature } & $-4.146 * * *$ & 0.000 & & $-6.120 * * *$ & $-5.541 * * *$ \\
\hline & $(0.86)$ & (.) & & $(0.80)$ & $(0.63)$ \\
\hline \multirow[t]{2}{*}{ Wealth (log of GDP) } & 0.021 & $-0.739 * * *$ & & $-0.811 * * *$ & $-0.758^{* *}$ \\
\hline & $(0.40)$ & $(0.25)$ & & $(0.31)$ & $(0.34)$ \\
\hline \multirow[t]{2}{*}{ Age of Democracy (logged) } & 0.072 & -0.020 & & -0.007 & -0.003 \\
\hline & $(0.05)$ & $(0.02)$ & & $(0.02)$ & $(0.02)$ \\
\hline \multirow[t]{2}{*}{ Past Democratic Reversions } & 0.872 & 0.536 & & $0.874^{*}$ & 0.678 \\
\hline & $(0.77)$ & $(0.40)$ & & $(0.46)$ & $(0.54)$ \\
\hline \multirow[t]{2}{*}{ Economic Crisis } & 1.115 & -0.156 & & 0.231 & 0.089 \\
\hline & $(0.73)$ & $(0.38)$ & & $(0.44)$ & $(0.50)$ \\
\hline \multirow[t]{2}{*}{ Presence of Territorial Rivalry } & $1.107^{*}$ & -0.124 & & -0.217 & 0.168 \\
\hline & $(0.67)$ & $(0.44)$ & & $(0.52)$ & $(0.54)$ \\
\hline \multirow[t]{2}{*}{ Highest level of militarization among neighbors } & 0.718 & -0.091 & & 0.088 & 0.134 \\
\hline & $(1.03)$ & $(0.40)$ & & $(0.46)$ & $(0.51)$ \\
\hline \multirow[t]{2}{*}{ Constant } & 1.194 & 2.382 & & $8.839 * * *$ & $7.752 * *$ \\
\hline & $(5.02)$ & $(2.90)$ & & $(3.42)$ & $(3.72)$ \\
\hline $\mathrm{N}$ & 176 & 1815 & & 1939 & 905 \\
\hline Chi2 & 48.386 & 49.111 & & 235.415 & 152.346 \\
\hline Prob $>$ Chi2 & 0.000 & 0.000 & & 0.000 & 0.000 \\
\hline Pseudo-R2 & 0.402 & 0.145 & & 0.515 & 0.465 \\
\hline
\end{tabular}




\begin{tabular}{|c|c|c|c|c|}
\hline & $\begin{array}{c}\text { Reich } 2002 \\
\text { (hybrid) }\end{array}$ & $\begin{array}{l}\text { Skaaning et } \\
\text { al. } 2015\end{array}$ & $\begin{array}{l}\text { Wahman et } \\
\text { al. } 2013 \text { (any } \\
\text { change) }\end{array}$ & $\begin{array}{l}\text { Wahman et } \\
\text { al. } 2013 \\
\text { (1.5+ points) }\end{array}$ \\
\hline Established Judiciary & -0.066 & no thresholds & -0.377 & -0.423 \\
\hline Newly Formed Judiciary & 0.326 & AUT & -0.246 & 0.344 \\
\hline & $(1.16)$ & & $(0.38)$ & $(1.18)$ \\
\hline Independent Legislature & $\begin{array}{c}-3.936 * * * \\
(0.70)\end{array}$ & & 0.000 & 0.000 \\
\hline Wealth (log of GDP) & $\begin{array}{l}0.097 \\
(0.33)\end{array}$ & & $\begin{array}{c}-0.543^{* * *} \\
(0.14)\end{array}$ & $\begin{array}{c}-0.292 \\
(0.60)\end{array}$ \\
\hline Age of Democracy (logged) & $\begin{array}{l}0.039 \\
(0.04)\end{array}$ & & $\begin{array}{c}-0.012^{* *} \\
(0.01)\end{array}$ & $\begin{array}{l}-0.005 \\
(0.02)\end{array}$ \\
\hline Past Democratic Reversions & $\begin{array}{l}0.596 \\
(0.61)\end{array}$ & & $\begin{array}{c}0.490 * * \\
(0.24)\end{array}$ & $\begin{array}{l}1.111 \\
(0.90)\end{array}$ \\
\hline Economic Crisis & $\begin{array}{l}0.353 \\
(0.60)\end{array}$ & & $\begin{array}{l}-0.064 \\
(0.22)\end{array}$ & $\begin{array}{l}1.408 \\
(0.94)\end{array}$ \\
\hline Presence of Territorial Rivalry & $\begin{array}{c}1.200 * * \\
(0.58)\end{array}$ & & $\begin{array}{l}0.158 \\
(0.25)\end{array}$ & $\begin{array}{c}-0.423 \\
(1.22)\end{array}$ \\
\hline Highest level of militarization among neighbors & $\begin{array}{l}0.855 \\
(0.67)\end{array}$ & & $\begin{array}{c}-0.175 \\
(0.22)\end{array}$ & $\begin{array}{c}-3.677 * * \\
(1.63)\end{array}$ \\
\hline Constant & $\begin{array}{l}1.337 \\
(4.29)\end{array}$ & & $\begin{array}{l}2.033 \\
(1.65)\end{array}$ & $\begin{array}{c}-19.981 * * \\
(9.16)\end{array}$ \\
\hline $\mathrm{N}$ & 350 & & 1270 & 1270 \\
\hline Chi2 & 60.631 & & 85.883 & 15.634 \\
\hline Prob $>$ Chi2 & 0.000 & & 0.000 & 0.048 \\
\hline Pseudo-R2 & 0.369 & & 0.111 & 0.205 \\
\hline
\end{tabular}




\section{Appendix XII: Robustness Checks: Goldstone et al. 2010}

a) all potential reformers

\begin{tabular}{|c|c|c|c|c|}
\hline & $\begin{array}{l}\text { Freedom } \\
\text { House } \\
\text { (FIW: any } \\
\text { change) }\end{array}$ & $\begin{array}{c}\text { Fredom } \\
\text { House (PR: } \\
\text { any change) }\end{array}$ & $\begin{array}{l}\text { Freedom } \\
\text { House } \\
\text { (FIW: 1+ } \\
\text { points) }\end{array}$ & $\begin{array}{c}\text { Freedom } \\
\text { House (PR: } \\
\text { 1+ points) }\end{array}$ \\
\hline Partial Autocracy & $\begin{array}{l}1.095 \\
(1.47)\end{array}$ & $\begin{array}{l}0.854 \\
(1.55)\end{array}$ & $\begin{array}{l}1.925 \\
(1.54)\end{array}$ & $\begin{array}{l}0.854 \\
(1.55)\end{array}$ \\
\hline Partial Democracy with Factionalism & $\begin{array}{c}5.279 * * \\
(2.08)\end{array}$ & $\begin{array}{c}6.069 * * \\
(2.59)\end{array}$ & $\begin{array}{c}5.463^{* * *} \\
(2.11)\end{array}$ & $\begin{array}{c}6.069^{* *} \\
(2.59)\end{array}$ \\
\hline Partial Democracy without Factionalism & $\begin{array}{l}1.610 \\
(1.53)\end{array}$ & $\begin{array}{l}1.769 \\
(1.51)\end{array}$ & $\begin{array}{l}1.906 \\
(1.49)\end{array}$ & $\begin{array}{l}1.769 \\
(1.51)\end{array}$ \\
\hline Full Democracy & $\begin{array}{c}7.269 * * \\
(3.20)\end{array}$ & $\begin{array}{l}4.138 \\
(6.96)\end{array}$ & $\begin{array}{l}4.185 \\
(3.96)\end{array}$ & $\begin{array}{l}4.138 \\
(6.96)\end{array}$ \\
\hline Infant mortality & $\begin{array}{c}4.084^{* *} \\
(1.96)\end{array}$ & $\begin{array}{c}5.066^{* *} \\
(2.50)\end{array}$ & $\begin{array}{c}3.559 * \\
(1.93)\end{array}$ & $\begin{array}{c}5.066^{* *} \\
(2.50)\end{array}$ \\
\hline Armed conflict in $4+$ Bordering States & $\begin{array}{c}15.873 \\
(3144.58)\end{array}$ & $\begin{array}{c}12.642 \\
(6801.26)\end{array}$ & & $\begin{array}{c}12.642 \\
(6801.26)\end{array}$ \\
\hline State-Led Discrimination & $\begin{array}{r}-1.327 \\
(1.09)\end{array}$ & $\begin{array}{r}-0.849 \\
(1.12)\end{array}$ & $\begin{array}{c}-0.653 \\
(1.03)\end{array}$ & $\begin{array}{c}-0.849 \\
(1.12)\end{array}$ \\
\hline $\mathrm{N}$ & 66 & 63 & 52 & 63 \\
\hline Chi2 & 30.265 & 31.631 & 23.141 & 31.631 \\
\hline Prob $>$ Chi2 & 0.000 & 0.000 & 0.001 & 0.000 \\
\hline Pseudo-R2 & 0.625 & 0.702 & 0.634 & 0.702 \\
\hline
\end{tabular}

\begin{tabular}{|l|ccccc|}
\hline & $\begin{array}{c}\text { Gasiorowski } \\
\mathbf{1 9 9 6} \\
\text { (hybrid) }\end{array}$ & $\begin{array}{c}\text { Gates et al. } \\
\mathbf{2 0 0 6} \text { (any } \\
\text { change) }\end{array}$ & $\begin{array}{c}\text { Polity IV } \\
\text { (any } \\
\text { change) }\end{array}$ & $\begin{array}{c}\text { Polity IV } \\
\text { (3+ points) }\end{array}$ & $\begin{array}{c}\text { Polity IV } \\
\text { (5+ points) }\end{array}$ \\
\hline Partial Autocracy & not concave & 1.805 & $3.760^{* * *}$ & $3.434^{* *}$ & 0.573 \\
Partial Democracy with Factionalism & & $(1.21)$ & $(1.12)$ & $(1.38)$ & $(1.57)$ \\
& & $3.898^{* * *}$ & $5.350^{* * *}$ & $5.425^{* * *}$ & $2.728^{*}$ \\
Partial Democracy without Factionalism & & $(1.32)$ & $(1.16)$ & $(1.42)$ & $(1.40)$ \\
& & 1.677 & $3.160^{* * *}$ & $3.221^{* *}$ & 0.193 \\
Full Democracy & & $(1.05)$ & $(1.07)$ & $(1.26)$ & $(1.39)$ \\
& & -15.569 & $2.510^{*}$ & 2.354 & -0.183 \\
Infant mortality & & $(3789.08)$ & $(1.28)$ & $(1.64)$ & $(1.76)$ \\
& & $1.234 *$ & $1.471 * *$ & $1.645^{* *}$ & $1.807^{* *}$ \\
Armed conflict in 4+ Bordering States & & $(0.74)$ & $(0.58)$ & $(0.65)$ & $(0.78)$ \\
State-Led Discrimination & & -0.394 & -1.090 & -1.367 & 0.000 \\
& & $(7645.49)$ & $(1.75)$ & $(1.92)$ & $()$. \\
N & & 0.390 & -0.814 & -1.074 & -0.867 \\
Chi2 & & $(0.97)$ & $(0.63)$ & $(0.76)$ & $(0.81)$ \\
Prob>Chi2 & & 81 & 185 & 148 & 98 \\
Pseudo-R2 & & 31.130 & 78.082 & 63.179 & 34.820 \\
\end{tabular}




\begin{tabular}{|c|c|c|c|c|}
\hline & $\begin{array}{c}\text { Reich } 2002 \\
\text { (hybrid) }\end{array}$ & $\begin{array}{l}\text { Skaaning et } \\
\text { al. } 2015\end{array}$ & $\begin{array}{l}\text { Wahman et } \\
\text { al. } 2013 \text { (any } \\
\text { change) }\end{array}$ & $\begin{array}{c}\text { Wahman et } \\
\text { al. } 2013 \\
(1.5+\text { points })\end{array}$ \\
\hline Partial Autocracy & not concave & $\begin{array}{c}-16.009 \\
(7824.06)\end{array}$ & $\begin{array}{l}1.568 \\
(1.42)\end{array}$ & not concave \\
\hline Partial Democracy with Factionalism & & $\begin{array}{c}3.875^{* * *} \\
(1.33)\end{array}$ & $\begin{array}{c}4.929 * * * \\
(1.73)\end{array}$ & \\
\hline Partial Democracy without Factionalism & & $\begin{array}{l}1.596 \\
(1.40)\end{array}$ & $\begin{array}{c}2.834^{*} \\
(1.51)\end{array}$ & \\
\hline Full Democracy & & $\begin{array}{c}-12.346 \\
(5591.16)\end{array}$ & $\begin{array}{c}4.678^{*} \\
(2.53)\end{array}$ & \\
\hline Infant mortality & & $\begin{array}{l}2.043 \\
(1.93)\end{array}$ & $\begin{array}{c}2.255^{* *} \\
(1.02)\end{array}$ & \\
\hline Armed conflict in $4+$ Bordering States & & $\begin{array}{c}-16.980 \\
(4971.68)\end{array}$ & $\begin{array}{c}-0.536 \\
(1.68)\end{array}$ & \\
\hline State-Led Discrimination & & $\begin{array}{c}-0.009 \\
(1.26)\end{array}$ & $\begin{array}{l}-1.403 \\
(0.96)\end{array}$ & \\
\hline $\mathrm{N}$ & & 67 & 80 & \\
\hline Chi2 & & 33.309 & 31.530 & \\
\hline Prob $>$ Chi2 & & 0.000 & 0.000 & \\
\hline Pseudo-R2 & & 0.708 & 0.545 & \\
\hline
\end{tabular}

\begin{tabular}{|c|c|c|c|c|c|}
\hline & $\begin{array}{c}\text { V-Dem: } \\
\text { Electoral } \\
\text { Democracy }\end{array}$ & $\begin{array}{c}\text { V-Dem: } \\
\text { Liberal } \\
\text { Democracy }\end{array}$ & $\begin{array}{c}\text { V-Dem: } \\
\text { Participatory } \\
\text { Democracy }\end{array}$ & $\begin{array}{c}\text { V-Dem: } \\
\text { Deliberative } \\
\text { Democracy }\end{array}$ & $\begin{array}{c}\text { V-Dem: } \\
\text { Egalitarian } \\
\text { Democracy }\end{array}$ \\
\hline Partial Autocracy & $\begin{array}{c}1.704^{*} \\
(0.87)\end{array}$ & not concave & $\begin{array}{c}2.412 * * \\
(1.03)\end{array}$ & $\begin{array}{l}1.694 \\
(1.35)\end{array}$ & $\begin{array}{c}2.320^{* *} \\
(1.03)\end{array}$ \\
\hline Partial Democracy with Factionalism & $\begin{array}{c}3.080^{* * *} \\
(0.95)\end{array}$ & & $\begin{array}{c}2.712^{* * *} \\
(0.86)\end{array}$ & $\begin{array}{c}3.249 * * * \\
(1.24)\end{array}$ & $\begin{array}{c}3.202^{* * *} \\
(1.05)\end{array}$ \\
\hline Partial Democracy without Factionalism & $\begin{array}{c}1.921^{* *} \\
(0.93)\end{array}$ & & $\begin{array}{c}2.584^{* *} \\
(1.03)\end{array}$ & $\begin{array}{c}2.337^{*} \\
(1.24)\end{array}$ & $\begin{array}{c}2.320^{* *} \\
(1.01)\end{array}$ \\
\hline Full Democracy & $\begin{array}{l}0.815 \\
(1.19)\end{array}$ & & $\begin{array}{c}2.950^{*} \\
(1.55)\end{array}$ & $\begin{array}{l}1.010 \\
(1.92)\end{array}$ & $\begin{array}{l}1.937 \\
(1.39)\end{array}$ \\
\hline Infant mortality & $\begin{array}{l}0.541 \\
(0.61)\end{array}$ & & $\begin{array}{c}1.418^{*} \\
(0.83)\end{array}$ & $\begin{array}{l}1.421 \\
(1.01)\end{array}$ & $\begin{array}{l}0.817 \\
(0.65)\end{array}$ \\
\hline Armed conflict in $4+$ Bordering States & $\begin{array}{c}14.481 \\
(2247.90)\end{array}$ & & $\begin{array}{c}-16.781 \\
(3187.08)\end{array}$ & $\begin{array}{c}14.069 \\
(4187.88)\end{array}$ & $\begin{array}{c}14.347 \\
(1886.29)\end{array}$ \\
\hline State-Led Discrimination & $\begin{array}{c}-0.196 \\
(0.74)\end{array}$ & & $\begin{array}{l}0.460 \\
(0.70)\end{array}$ & $\begin{array}{c}-0.199 \\
(0.83)\end{array}$ & $\begin{array}{c}-0.388 \\
(0.84)\end{array}$ \\
\hline $\mathrm{N}$ & 89 & & 87 & 68 & 77 \\
\hline Chi2 & 23.757 & & 22.944 & 24.234 & 21.420 \\
\hline Prob $>$ Chi2 & 0.003 & & 0.002 & 0.002 & 0.003 \\
\hline Pseudo-R2 & 0.373 & & 0.372 & 0.491 & 0.387 \\
\hline
\end{tabular}




\begin{tabular}{|c|c|c|c|c|c|}
\hline & $\begin{array}{c}\text { V-Dem: } \\
\text { Electoral } \\
\text { Democracy } \\
\text { (ordinal) }\end{array}$ & $\begin{array}{l}\text { V-Dem: } \\
\text { Liberal } \\
\text { Democracy } \\
\text { (ordinal) }\end{array}$ & $\begin{array}{c}\text { V-Dem: } \\
\text { Participatory } \\
\text { Democracy } \\
\text { (ordinal) }\end{array}$ & $\begin{array}{l}\text { V-Dem: } \\
\text { Deliberative } \\
\text { Democracy } \\
\text { (ordinal) }\end{array}$ & $\begin{array}{l}\text { V-Dem: } \\
\text { Egalitarian } \\
\text { Democracy } \\
\text { (ordinal) }\end{array}$ \\
\hline Partial Autocracy & $\begin{array}{l}0.433 \\
(1.51)\end{array}$ & $\begin{array}{c}35.394 \\
(48632700.04)\end{array}$ & not concave & not concave & not concave \\
\hline Partial Democracy with Factionalism & $\begin{array}{c}19.976 \\
(5593.66)\end{array}$ & $\begin{array}{c}34.018 \\
(48632700.04)\end{array}$ & & & \\
\hline Partial Democracy without Factionalism & $\begin{array}{l}1.313 \\
(1.25)\end{array}$ & $\begin{array}{c}32.549 \\
(48632700.04)\end{array}$ & & & \\
\hline Full Democracy & $\begin{array}{l}-1.639 \\
(1.81)\end{array}$ & $\begin{array}{c}16.928 \\
(48632700.20)\end{array}$ & & & \\
\hline Infant mortality & $\begin{array}{l}0.309 \\
(0.95)\end{array}$ & $\begin{array}{l}2.341 \\
(2.15)\end{array}$ & & & \\
\hline Armed conflict in 4+ Bordering States & $\begin{array}{c}16.362 \\
(12681.42)\end{array}$ & $\begin{array}{l}0.000 \\
(.)\end{array}$ & & & \\
\hline State-Led Discrimination & $\begin{array}{r}-0.641 \\
(1.28)\end{array}$ & $\begin{array}{l}-0.578 \\
(1.64)\end{array}$ & & & \\
\hline $\mathrm{N}$ & 54 & 27 & & & \\
\hline Chi2 & 22.990 & 12.476 & & & \\
\hline Prob $>$ Chi2 & 0.003 & 0.014 & & & \\
\hline Pseudo-R2 & 0.571 & 0.620 & & & \\
\hline
\end{tabular}

b) DEM only

\begin{tabular}{|l|cccc|}
\hline & $\begin{array}{c}\text { Freedom } \\
\text { House } \\
\text { (FIW: any } \\
\text { change) }\end{array}$ & $\begin{array}{c}\text { Fredom } \\
\text { House (PR: } \\
\text { any change) }\end{array}$ & $\begin{array}{c}\text { Freedom } \\
\text { House } \\
\text { (FIW: 1+ } \\
\text { points) }\end{array}$ & $\begin{array}{c}\text { Freedom } \\
\text { House (PR: } \\
\text { 1+ points) }\end{array}$ \\
\hline Partial Autocracy & not concave & not concave & not concave & not concave \\
Partial Democracy without Factionalism & & & \\
Full Democracy & & & \\
Infant mortality & & & \\
Armed conflict in 4+ Bordering States & & & \\
State-Led Discrimination & & & \\
N & & & \\
Chi2 \\
Prob>Chi2 \\
Pseudo-R2
\end{tabular}




\begin{tabular}{|c|c|c|c|c|c|}
\hline & $\begin{array}{c}\text { Gasiorowski } \\
1996 \\
\text { (hybrid) }\end{array}$ & $\begin{array}{c}\text { Gates et al. } \\
2006 \text { (any } \\
\text { change) }\end{array}$ & $\begin{array}{l}\text { Polity IV } \\
\text { (any } \\
\text { change) }\end{array}$ & $\begin{array}{c}\text { Polity IV } \\
(3+\text { points })\end{array}$ & $\begin{array}{c}\text { Polity IV } \\
\text { (5+ points) }\end{array}$ \\
\hline Partial Autocracy & not concave & not concave & $\begin{array}{l}2.788 \\
(2.68)\end{array}$ & $\begin{array}{l}1.890 \\
(2.80)\end{array}$ & $\begin{array}{c}-32.585 \\
(28969612.04)\end{array}$ \\
\hline Partial Democracy with Factionalism & & & $\begin{array}{l}2.103 \\
(2.09)\end{array}$ & $\begin{array}{l}1.983 \\
(2.01)\end{array}$ & $\begin{array}{c}-32.179 \\
(28969612.04)\end{array}$ \\
\hline Partial Democracy without Factionalism & & & $\begin{array}{c}-18.244 \\
(6723.31)\end{array}$ & $\begin{array}{c}-18.348 \\
(8058.92)\end{array}$ & $\begin{array}{c}-52.012 \\
(28969613.08)\end{array}$ \\
\hline Full Democracy & & & $\begin{array}{c}-51.839 \\
(12538.36)\end{array}$ & $\begin{array}{c}-52.477 \\
(14791.67)\end{array}$ & $\begin{array}{c}-50.849 \\
(28969612.70)\end{array}$ \\
\hline Infant mortality & & & $\begin{array}{c}1.577^{*} \\
(0.88)\end{array}$ & $\begin{array}{c}1.643^{*} \\
(0.91)\end{array}$ & $\begin{array}{c}1.566^{*} \\
(0.87)\end{array}$ \\
\hline Armed conflict in $4+$ Bordering States & & & $\begin{array}{c}-17.783 \\
(7971.39)\end{array}$ & $\begin{array}{c}-18.020 \\
(9092.26)\end{array}$ & $\begin{array}{c}0.000 \\
(.)\end{array}$ \\
\hline State-Led Discrimination & & & $\begin{array}{c}-2.314 \\
(1.59)\end{array}$ & $\begin{array}{c}-1.998 \\
(1.57)\end{array}$ & $\begin{array}{l}-1.468 \\
(1.52)\end{array}$ \\
\hline $\mathrm{N}$ & & & 81 & 77 & 68 \\
\hline Chi2 & & & 40.909 & 38.801 & 33.452 \\
\hline Prob $>$ Chi2 & & & 0.000 & 0.000 & 0.000 \\
\hline Pseudo-R2 & & & 0.712 & 0.710 & 0.692 \\
\hline
\end{tabular}

\begin{tabular}{|c|c|c|c|c|}
\hline & $\begin{array}{c}\text { Reich } 2002 \\
\text { (hybrid) }\end{array}$ & $\begin{array}{l}\text { Skaaning et } \\
\text { al. } 2015\end{array}$ & $\begin{array}{c}\text { Wahman et } \\
\text { al. } 2013 \text { (any } \\
\text { change) }\end{array}$ & $\begin{array}{c}\text { Wahman et } \\
\text { al. } 2013 \\
\text { (1.5+ points) }\end{array}$ \\
\hline Partial Autocracy & not concave & $\begin{array}{l}\text { no thresholds } \\
\text { for DEM/ }\end{array}$ & not concave & not concave \\
\hline Partial Democracy with Factionalism & & AUT & & \\
\hline \multicolumn{5}{|l|}{ Partial Democracy without Factionalism } \\
\hline \multicolumn{5}{|l|}{ Full Democracy } \\
\hline \multicolumn{5}{|l|}{ Infant mortality } \\
\hline \multicolumn{5}{|l|}{ Armed conflict in $4+$ Bordering States } \\
\hline \multicolumn{5}{|l|}{ State-Led Discrimination } \\
\hline $\mathrm{N}$ & & & & \\
\hline Chi2 & & & & \\
\hline Prob $>$ Chi2 & & & & \\
\hline Pseudo-R2 & & & & \\
\hline
\end{tabular}




\section{References}

Alvarez, Michael, José A. Cheibub, Fernando Limongi and Adam Przeworski. 1996.

“Classifying Political Regimes.” Studies in Comparative International Development 31(2): 336.

Bernhard, Michael, Timothy Nordstrom and Christopher Reenock. 2001. "Economic Performance, Institutional Intermediation and Democratic Breakdown." Journal of Politics 63(3): 775-803.

Bernhard, Michael, Christopher Reenock and Timothy Nordstrom. 2003. "Economic Performance and Survival in New Democracies: Is There a Honeymoon Effect?” Comparative Political Studies 36(4): 404-431.

Bernhard, Michael, Christopher Reenock and Timothy Nordstrom. 2004. "The Legacy of Western Overseas Colonialism on Democratic Survival.” International Studies Quarterly 48(1): 225-250.

Boix, Carles. 2003. Democracy and Redistribution. Cambridge, UK: Cambridge University Press.

Boix, Carles, Michael K. Miller and Sebastian Rosato. 2013. “A Complete Data Set of Political Regimes, 1800-2007." Comparative Political Studies 46(12): 1523-1554.

Brambor, Thomas and Johannes Lindvall. 2014. "Democratic Breakdowns in Economic Crises: The Role of Political Ideology." http://www.reformcapacity.org/uploads/1/3/7/5/13752394/brambor.lindvall.2014 .democratic breakdowns.pdf (retrieved 10/12/2014).

Brinks, Daniel, and Michael Coppedge. 2006. "Diffusion Is No Illusion: Neighbor Emulation in the Third Wave of Democracy." Comparative Political Studies 39(4): 463489.

Burke, Paul J, and Andrew Leigh. 2010. "Do Output Contractions Trigger Democratic Change?" American Economic Journal: Macroenomics 2(4): 124-157.

Cheibub, José A. 2007. Presidentialism, Parliamentarism, and Democracy. Cambridge, UK: Cambridge University Press.

Cheibub, José A., Jennifer Gandhi and James R. Vreeland. 2010. "Democracy and Dictatorship Revisited." Public Choice 143(1-2): 67-101.

Coppedge, Michael, John Gerring, Staffan I. Lindberg, Svend-Erik Skaaning, Jan Teorell, David Altman, Michael Bernhard, M. Steven Fish, Adam Glynn, Allen Hicken, Carl 
Henrik Knutsen, Kyle Marquardt, Kelly McMann, Farhad Miri, Pamela Paxton, Daniel Pemstein, Jeffrey Staton, Eitan Tzelgov, Yi-ting Wang, and Brigitte Zimmerman. 2016a. "V-Dem [Country-Year/Country-Date] Dataset v6.2." Gothenburg, Sweden: Varieties of Democracy (V-Dem) Project. https://www.vdem.net/en/data/data-version-6-2/ (retrieved 10/30/2016).

Epstein, David L., Robert Bates, Jack Goldstone, Ida Kristensen and Sharyn O'Halloran. 2006. “Democratic Transitions.” American Journal of Political Science 50(3): 551-569.

Erdmann, Gero. 2011. "Decline of Democracy: Loss of Quality, Hybridisation and Breakdown of Democracy." Zeitschrift für Vergleichende Politikwissenschaft. Comparative Politics and Governance special issue 1/2011: 21-58.

Fjelde, Hanne and Håvard Hegre. 2006. "Democracy Depraved: Corruption and Institutional Change 1985-2000.” http://www.svt.ntnu.no/iss/fagkonferanse2007/intern/papers/hanne.fjelde@pcr.u u.secorr stab201206.pdf (retrieved 10/12/2014).

Freedom House. 2015. Freedom in the World. Washington, DC: Freedom House. https://freedomhouse.org/report/freedom-world/freedom-world-2015 (retrieved 02/04/2016).

Gasiorowski, Mark J. 1996. "An Overview of the Political Regime Change Dataset.” Comparative Political Studies 29(4): 469-483.

Gasiorowski, Mark J. and Timothy J. Power. 1998. "The Structural Determinants of Democratic Consolidation: Evidence from the Third World." Comparative Political Studies 31(6): 740-771.

Gates, Scott, Håvard Hegre, Mark P. Jones and Håvard Strand. 2006. "Institutional Inconsistency and Instability: Polity Duration, 1800-2000.” American Journal of Political Science 50(4): 893-908.

Geddes, Barbara, Joseph Wright and Erica Frantz. 2014. "Autocratic Breakdown and Regime Transitions: A New Data Set.” Perspectives on Politics 12(2): 313-331.

Gibler, Douglas M. and Kirk A. Randazzo. 2011. "Testing the Effects of Independent Judiciaries on the Likelihood of Democratic Backsliding." American Journal of Political Science 55(3): 696-709. 
Goldstone, Jack A., Robert Bates, David L. Epstein, Ted R. Gurr, Michael B. Lustik, Monty G. Marshall, Jay Ulfelder and Mark Woodward. 2010. “A Global Model for Forecasting Political Instability." American Journal of Political Science 54(1): 190-208.

Houle, Christian. 2009. "Inequality and Democracy: Why Inequality Harms Consolidation but Does Not Affect Democratization.” World Politics 61(4): 589-622.

Kadera, Kelly M., Mark J.C. Crescenzi and Megan L. Shannon. 2003. "Democratic Survival, Peace, and War in the International System." American Journal of Political Science 47(2): 234-247.

Kapstein, Ethan B., and Nathan Converse (2008): The Fate of Young Democracies. Cambridge, UK: Cambridge University Press.

Lai, Brian and Ruth Melkonian-Hoover. 2005. "Democratic Progress and Regress: The Effect of Parties on the Transitions of States to and Away from Democracy." Political Research Quarterly 58(4): 551-564.

Levitz, Philip and Grigore Pop-Eleches. 2010. "Why No Backsliding? The European Union's Impact on Democracy and Governance Before and After Accession.” Comparative Political Studies 43(4): 457-485.

Maeda, Ko. 2010. “Two Modes of Democratic Breakdown: A Competing Risks Analysis of Democratic Durability.” Journal of Politics 72(4): 1129-1143.

Magaloni, Beatriz, Jonathan Chu and Eric Min. 2013. Autocracies of the World, 1950-2012 (Version 1.0). Dataset. Stanford, CA: Stanford University. http://cddrl.fsi.stanford.edu/research/autocracies_of_the_world_dataset (retrieved 10/30/2014).

Marshall, Monty G., Keith Jaggers and Ted R. Gurr. 2014. Polity IV Annual Time-Series 18002013. http://www.systemicpeace.org/(retrieved 10/24/2014).

Pevehouse, Jon. 2002. "Democracy from the Outside-In? International Organizations and Democratization.” International Organization 56(3): 515-549.

Power, Timothy J. and Mark J. Gasiorowski. 1997. "Institutional Design and Democratic Consolidation in the Third World." Comparative Political Studies 30(2): 123-155.

Przeworski, Adam, Michael Alvarez, José A. Cheibub and Fernando Limongi. 2000. Democracy and Development: Political Institutions and Material Well-Being in the World, 19501990. Cambridge, UK: Cambridge University Press. 
Reenock, Christopher, Michael Bernhard and David Sobek. 2007. "Regressive Socioeconomic Distribution and Democratic Survival.” International Studies Quarterly 51: 677-699.

Reich, Gary. 2002. “Categorizing Political Regimes: New Data for Old Problems.” Democratization 9(4): 1-24.

Shirah, Ryan. 2013. "Authoritarian Backsliding in New Democracies." https://wpsa.research.pdx.edu/papers/docs/shirah\%20wpsa13.pdf (retrieved 11/02/2016).

Skaaning, Svend-Erik, John Gerring and Henrikas Bartusevičius. 2015. “A Lexical Index of Electoral Democracy." Comparative Political Studies 48(12): 1491-1525.

Starr, Harvey and Christina Lindborg. 2003. "Democratic Dominoes Revisited: The Hazards of Governmental Transitions, 1974-1996." Journal of Conflict Resolution 47(4): 490-519.

Strand, Håvard, Håvard Hegre, Scott Gates and Marianne Dahl. 2013. “Why Waves? Global Patterns of Democratization, 1816-2008.” http://folk.uio.no/hahegre/Papers/WhyWaves 2012.pdf (retrieved 11/02/2016).

Svolik, Milan. 2008. "Authoritarian Reversals and Democratic Consolidation.” American Political Science Review 102(2): 153-168.

Svolik, Milan. 2012. The Politics of Authoritarian Rule. New York, NY: Cambridge University Press.

Ulfelder, Jay. 2012. Democracy/Autocracy Data Set. https://dataverse.harvard.edu/dataset.xhtml?persistentId=hdl:1902.1/18836 (retrieved 2015/05/12).

Ulfelder, Jay and Michael Lustik. 2007. "Modelling Transitions to and From Democracy." Democratization 14(3): 351-387.

Wahman, Michael, Jan Teorell and Axel Hadenius. 2013. “Authoritarian Regime Types Revisited: Updated Data in Comparative Perspective." Contemporary Politics 18(1): 1934.

Wright, Joseph and Abel Escribà-Folch. 2012. "Authoritarian Institutions and Regime Survival: Transitions to Democracy and Subsequent Authoritarian Regimes." British Journal of Political Science 42(2): 283-309. 